JOURNAL OF THE

AMERICAN MATHEMATICAL SOCIETY

Volume 16, Number 4, Pages 779-856

S 0894-0347(03)00434-X

Article electronically published on July 21, 2003

\title{
THE HOMOTOPY THEORY OF FUSION SYSTEMS
}

\author{
CARLES BROTO, RAN LEVI, AND BOB OLIVER
}

The main goal of this paper is to identify and study a certain class of spaces which in many ways behave like $p$-completed classifying spaces of finite groups. These spaces occur as the "classifying spaces" of certain algebraic objects, which we call $p$-local finite groups. A p-local finite group consists, roughly speaking, of a finite $p$-group $S$ and fusion data on subgroups of $S$, encoded in a way explained below. Our starting point is our earlier paper [BLO] on $p$-completed classifying spaces of finite groups, together with the axiomatic treatment by Lluís Puig $[\mathrm{Pu}$, Pu2 of systems of fusion among subgroups of a given $p$-group.

The $p$-completion of a space $X$ is a space $X_{p}^{\wedge}$ which isolates the properties of $X$ at the prime $p$, and more precisely the properties which determine its mod $p$ cohomology. For example, a map of spaces $X \stackrel{f}{\longrightarrow} Y$ induces a homotopy equivalence $X_{p}^{\wedge} \stackrel{\simeq}{\longrightarrow} Y_{p}^{\wedge}$ if and only if $f$ induces an isomorphism in mod $p$ cohomology; and $H^{*}\left(X_{p}^{\wedge} ; \mathbb{F}_{p}\right) \cong H^{*}\left(X ; \mathbb{F}_{p}\right)$ in favorable cases (if $X$ is " $p$-good"). When $G$ is a finite group, the $p$-completion $B G_{p}^{\wedge}$ of its classifying space encodes many of the properties of $G$ at $p$. For example, not only the $\bmod p$ cohomology of $B G$, but also the Sylow $p$-subgroup of $G$ together with all fusion among its subgroups, are determined up to isomorphism by the homotopy type of $B G_{p}^{\wedge}$.

Our goal here is to give a direct link between $p$-local structures and homotopy types which arise from them. This theory tries to make explicit the essence of what it means to be the $p$-completed classifying space of a finite group, and at the same time yields new spaces which are not of this type, but which still enjoy most of the properties a space of the form $B G_{p}^{\wedge}$ would have. We hope that the ideas presented here will have further applications and generalizations in algebraic topology. But this theory also fits well with certain aspects of modular representation theory. In particular, it may give a way of constructing classifying spaces for blocks in the group ring of a finite group over an algebraically closed field of characteristic $p$.

A saturated fusion system $\mathcal{F}$ over a p-group $S$ consists of a set $\operatorname{Hom}_{\mathcal{F}}(P, Q)$ of monomorphisms, for each pair of subgroups $P, Q \leq S$, which form a category under composition, include all monomorphisms induced by conjugation in $S$, and satisfy certain other axioms formulated by Puig (Definitions 1.1 and 1.2 below). In particular, these axioms are satisfied by the conjugacy homomorphisms in a finite group. We refer to $[\mathrm{Pu}]$ and $[\mathrm{Pu} 2]$ for more details of Puig's work on saturated

Received by the editors August 3, 2001.

2000 Mathematics Subject Classification. Primary 55R35; Secondary 55R40, 20D20.

Key words and phrases. Classifying space, $p$-completion, finite groups, fusion.

The first author is partially supported by MCYT grant BFM2001-2035.

The second author is partially supported by EPSRC grant GR/M7831.

The third author is partially supported by UMR 7539 of the CNRS.

All of the authors have been supported by EU grant HPRN-CT-1999-00119. 
fusion systems (which he calls "full Frobenius systems" in [Pu2]). The definitions and results given here, in Section 1 and in Appendix A are only a very brief account of those results of Puig used in our paper.

If $\mathcal{F}$ is a saturated fusion system over $S$, then two subgroups $P, P^{\prime} \leq S$ are called $\mathcal{F}$-conjugate if $\operatorname{Iso}_{\mathcal{F}}\left(P, P^{\prime}\right) \neq \emptyset$. A subgroup $P$ is called $\mathcal{F}$-centric if $C_{S}\left(P^{\prime}\right) \leq P^{\prime}$ for all $P^{\prime}$ that are $\mathcal{F}$-conjugate to $P$; this is equivalent to what Puig calls " $\mathcal{F}$ selfcentralizing". Let $\mathcal{F}^{c}$ be the full subcategory of $\mathcal{F}$ whose objects are the $\mathcal{F}$ centric subgroups of $S$. A centric linking system associated to $\mathcal{F}$ is a category $\mathcal{L}$ whose objects are the $\mathcal{F}$-centric subgroups of $S$, together with a functor $\mathcal{L} \stackrel{\pi}{\longrightarrow} \mathcal{F}^{c}$ which is the identity on objects and surjective on morphisms, and which satisfies other axioms listed below in Definition 1.7. For example, for each object $P$, the kernel of the induced map $\operatorname{Aut}_{\mathcal{L}}(P) \longrightarrow \operatorname{Aut}_{\mathcal{F}}(P)$ is isomorphic to $Z(P)$, and $\operatorname{Aut}_{\mathcal{L}}(P)$ contains a distinguished subgroup isomorphic to $P$.

The motivating examples for these definitions come from finite groups. If $G$ is a finite group and $p$ is a prime, then $\mathcal{F}=\mathcal{F}_{S}(G)$ is the fusion system over $S \in \operatorname{Syl}_{p}(G)$ such that for each $P, Q \leq S, \operatorname{Hom}_{\mathcal{F}}(P, Q)$ is the set of homomorphisms induced by conjugation in $G$ (and inclusion). The $\mathcal{F}$-centric subgroups of $S$ are the $p$-centric subgroups: those $P \leq S$ such that $C_{G}(P) \cong Z(P) \times C_{G}^{\prime}(P)$ for some $C_{G}^{\prime}(P)$ of order prime to $p$ (see [BLO Lemma A.5]). In [BLO], we defined a category $\mathcal{L}_{S}^{c}(G)$ whose objects are the $p$-centric subgroups of $G$ which are contained in $S$, and where $\operatorname{Mor}_{\mathcal{L}_{S}^{c}(G)}(P, Q)=N_{G}(P, Q) / C_{G}^{\prime}(P)$. Here, $N_{G}(P, Q)$ is the set of elements of $G$ which conjugate $P$ into $Q$. The category $\mathcal{L}_{S}^{c}(G)$, together with its projection to $\mathcal{F}_{S}(G)$ which sends the morphism corresponding to an element $g \in N_{G}(P, Q)$ to conjugation by $g$, is the example which motivated our definition of an associated centric linking system.

We define a $p$-local finite group to be a triple $(S, \mathcal{F}, \mathcal{L})$, where $\mathcal{L}$ is a centric linking system associated to a saturated fusion system $\mathcal{F}$ over a $p$-group $S$. The classifying space of such a triple is the space $|\mathcal{L}|_{p}^{\wedge}$, where for any small category $\mathcal{C}$, the space $|\mathcal{C}|$ denotes the geometric realization of the nerve of $\mathcal{C}$. This is partly motivated by the result that $\left|\mathcal{L}_{p}^{c}(G)\right|_{p}^{\wedge} \simeq B G_{p}^{\wedge}$ for any finite $G$ [BLO, Proposition 1.1]. But additional motivation comes from Proposition 2.2 below, which says that if $\mathcal{L}$ is a centric linking system associated to $\mathcal{F}$, then $|\mathcal{L}| \simeq{\underset{\text { hocolim}}{\longrightarrow}}_{\mathcal{O}^{c}(\mathcal{F})}(\widetilde{B})$, where $\mathcal{O}^{c}(\mathcal{F})$ is a certain quotient "orbit" category of $\mathcal{F}^{c}$, and $\widetilde{B}$ is a lifting of the homotopy functor which sends $P$ to $B P$. The classifying space of a $p$-local finite group thus comes equipped with a decomposition as the homotopy colimit of a finite diagram of classifying spaces of $p$-groups.

We now state our main results. Our first result is that a $p$-local finite group is determined up to isomorphism by its classifying space. What is meant by an isomorphism of $p$-local finite groups will be explained later.

Theorem A (Theorem 7.4). A p-local finite group $(S, \mathcal{F}, \mathcal{L})$ is determined by the homotopy type of $|\mathcal{L}|_{p}^{\wedge}$. In particular, if $(S, \mathcal{F}, \mathcal{L})$ and $\left(S^{\prime}, \mathcal{F}^{\prime}, \mathcal{L}^{\prime}\right)$ are two p-local finite groups and $|\mathcal{L}|_{p}^{\wedge} \simeq\left|\mathcal{L}^{\prime}\right|_{p}^{\wedge}$, then $(S, \mathcal{F}, \mathcal{L})$ and $\left(S^{\prime}, \mathcal{F}^{\prime}, \mathcal{L}^{\prime}\right)$ are isomorphic.

Next we study the cohomology of $p$-local finite groups. As one might hope, we have the following result, which appears as Theorem 5.8.

Theorem B. For any p-local finite group $(S, \mathcal{F}, \mathcal{L}), H^{*}\left(|\mathcal{L}|_{p}^{\wedge} ; \mathbb{F}_{p}\right)$ is isomorphic to the ring of "stable elements" in $H^{*}\left(B S ; \mathbb{F}_{p}\right)$; i.e., the inverse limit of the rings $H^{*}\left(B P ; \mathbb{F}_{p}\right)$ as a functor on the category $\mathcal{F}$. Furthermore, this ring is noetherian. 
The next theorem gives an explicit description of the mapping space from the classifying space of a finite $p$-group into the classifying space of a $p$-local finite group. It is stated precisely as Corollary 4.5 and Theorem 6.3

Theorem C. For any p-local finite group $(S, \mathcal{F}, \mathcal{L})$, and any p-group $Q$,

$$
\left[B Q,|\mathcal{L}|_{p}^{\wedge}\right] \cong \operatorname{Rep}(Q, \mathcal{L}) \stackrel{\text { def }}{=} \operatorname{Hom}(Q, S) /(\mathcal{F} \text {-conjugacy }) .
$$

Furthermore, each component of the mapping space has the homotopy type of the classifying space of a p-local finite group which can be thought of as the "centralizer" of the image of the corresponding homomorphism $Q \longrightarrow S$.

The next result describes the space of self equivalences of the classifying space of a $p$-local finite group. It is a generalization of [BLO Theorem C]. For a small category $\mathcal{C}$, let $\mathcal{A} u t(\mathcal{C})$ denote the groupoid whose objects are self equivalences of $\mathcal{C}$, and whose morphisms are natural isomorphisms of functors. Let $\mathcal{L}$ be a centric linking system associated to a saturated fusion system $\mathcal{F}$. Self equivalences of $\mathcal{L}$ which are structure preserving, in a sense to be made precise in section 7 below, are said to be isotypical. We let $\mathcal{A} u t_{\text {typ }}(\mathcal{L})$ denote the subgroupoid of $\mathcal{A} u t(\mathcal{L})$ whose objects are the isotypical self equivalences of $\mathcal{L}$. For a space $X$, let $\operatorname{Aut}(X)$ denote the topological monoid of all self homotopy equivalences of $X$. The following theorem is restated below as Theorem 8.1 .

Theorem D. Fix a p-local finite group $(S, \mathcal{F}, \mathcal{L})$. Then $\operatorname{Aut}\left(|\mathcal{L}|_{p}^{\wedge}\right)$ and $\left|\mathcal{A} u t_{\text {typ }}(\mathcal{L})\right|$ are equivalent as topological monoids in the sense that their classifying spaces are homotopy equivalent. In particular, their groups of components are isomorphic, and each component of $\operatorname{Aut}\left(|\mathcal{L}|_{p}^{\wedge}\right)$ is aspherical.

The statement of Theorem 8.1 also includes a description of the homotopy groups of $\operatorname{Aut}\left(|\mathcal{L}|_{p}^{\wedge}\right)$.

So far, we have not mentioned the question of the existence and uniqueness of centric linking systems associated to a given saturated fusion system. Of course, as pointed out above, any finite group $G$ gives rise to an associated $p$-local finite group. However there are saturated fusion systems which do not occur as the fusion system of any finite group. Thus a tool for deciding existence and uniqueness would be useful. The general obstructions to the existence and uniqueness of associated centric linking systems, which lie in certain higher limits taken over the orbit category $\mathcal{O}^{c}(\mathcal{F})$ of the fusion system, are described in Proposition 3.1; and a means of computing these groups is provided by Proposition 3.2 The following result is just one special consequence of this, which settles the question for $p$-groups of small rank. Here, for any finite group $G$, we write $\operatorname{rk}_{p}(G)$ for the largest rank of any elementary abelian $p$-subgroup of $G$.

Theorem E (Corollary 3.5). Fix a saturated fusion system $\mathcal{F}$ over a p-group $S$. If $\operatorname{rk}_{p}(S)<p^{3}$, then there exists a centric linking system associated to $\mathcal{F}$, and if $\operatorname{rk}_{p}(S)<p^{2}$, then the associated centric linking system is unique.

In the last section, we present some direct constructions of saturated fusion systems and associated $p$-local finite groups (see Examples 9.3 and 9.4). The idea is to look at the fusion system over a $p$-group $S$ (for $p$ odd only) generated by groups of automorphisms of $S$ and certain of its subgroups, and show that under certain hypotheses the resulting system is saturated. In all of these cases, the $p$-group $S$ is 
nonabelian, and has an index $p$ subgroup which is abelian and homocyclic (a product of cyclic groups of the same order). We then give a list of all finite simple groups which have Sylow subgroups of this form, based on the classification theorem, and use that to show that certain of the fusion systems which were constructed are not the fusion systems of any finite groups. In all cases, TheoremEapplies to show the existence and uniqueness of centric linking systems, and hence $p$-local finite groups, associated to these fusion systems.

The basic definitions of saturated fusion systems and their associated centric linking systems are given in Section 1. Homotopy decompositions of classifying spaces of $p$-local finite groups are constructed in Section 2. The obstruction theory for the existence and uniqueness of associated centric linking systems, as well as some results about those obstruction groups, are shown in Section 3. Maps from the classifying space of a $p$-group to the classifying space of a $p$-local finite group are studied in Sections 4 and 6, while the cohomology rings of classifying spaces of $p$-local finite groups are dealt with in Section 5. A characterization of classifying spaces of $p$-local finite groups is given in Section 7, and their spaces of self equivalences are described in Section 8. The "exotic" examples of $p$-local finite groups are constructed in Section 9. Finally, some additional results on saturated fusion systems are collected in an appendix.

We would like to thank Dave Benson and Jesper Grodal for their many suggestions throughout the course of this work. In particular, Dave had earlier written and distributed notes which contained some of the ideas of our centric linking systems. We would also like to thank Lluís Puig for giving us a copy of his unpublished notes on saturated fusion systems. Markus Linckelmann, Haynes Miller, Bill Dwyer, and Jon Alperin have all shown interest and made helpful comments and suggestions. Kasper Andersen and Kari Ragnarsson both read earlier versions of this paper in detail, and sent us many suggestions for improvements. Two of the authors would also like to thank Slain's Castle, a pub in Aberdeen, for their hospitality on New Year's Day while we worked out the proof that the nerve of a centric linking system is $p$-good.

We would especially like to thank the universities of Aberdeen and Paris-Nord, the CRM and the UAB in Barcelona, and the Max-Planck Institut in Bonn for their hospitality in helping the three authors get together in various combinations; and also the European Homotopy Theory Network for helping to finance these visits.

\section{Fusion Systems AND ASSOCIATED CENTRIC LINKING SYSTEMS}

We begin with the precise definitions of saturated fusion systems and their associated centric linking systems. Additional results about fusion systems due to Puig $[\mathrm{Pu}$, [Pu2] are in Appendix $\mathrm{A}$.

Given two finite groups $P, Q$, let $\operatorname{Hom}(P, Q)$ denote the set of group homomorphisms from $P$ to $Q$, and let $\operatorname{Inj}(P, Q)$ denote the set of monomorphisms. If $P$ and $Q$ are subgroups of a larger group $G$, then $\operatorname{Hom}_{G}(P, Q) \subseteq \operatorname{Inj}(P, Q)$ denotes the subset of homomorphisms induced by conjugation by elements of $G$, and $\operatorname{Aut}_{G}(P)$ the group of automorphisms induced by conjugation in $G$.

Definition 1.1. A fusion system $\mathcal{F}$ over a finite $p$-group $S$ is a category whose objects are the subgroups of $S$, and whose morphism sets $\operatorname{Hom}_{\mathcal{F}}(P, Q)$ satisfy the following conditions: 
(a) $\operatorname{Hom}_{S}(P, Q) \subseteq \operatorname{Hom}_{\mathcal{F}}(P, Q) \subseteq \operatorname{Inj}(P, Q)$ for all $P, Q \leq S$.

(b) Every morphism in $\mathcal{F}$ factors as an isomorphism in $\mathcal{F}$ followed by an inclusion.

Note that what we call a fusion system here is what Puig calls a divisible Frobenius system.

If $\mathcal{F}$ is a fusion system over $S$ and $P, Q \leq S$, then we write $\operatorname{Hom}_{\mathcal{F}}(P, Q)=$ $\operatorname{Mor}_{\mathcal{F}}(P, Q)$ to emphasize that morphisms in the category $\mathcal{F}$ are all homomorphisms, and $\operatorname{Iso}_{\mathcal{F}}(P, Q)$ for the subset of isomorphisms in $\mathcal{F}$. Thus $\operatorname{Iso}_{\mathcal{F}}(P, Q)=$ $\operatorname{Hom}_{\mathcal{F}}(P, Q)$ if $|P|=|Q|$, and $\operatorname{Iso}_{\mathcal{F}}(P, Q)=\emptyset$ otherwise. Also, $\operatorname{Aut}_{\mathcal{F}}(P)=$ $\operatorname{Iso}_{\mathcal{F}}(P, P)$ and $\operatorname{Out}_{\mathcal{F}}(P)=\operatorname{Aut}_{\mathcal{F}}(P) / \operatorname{Inn}(P)$. Two subgroups $P, P^{\prime} \leq S$ are called $\mathcal{F}$-conjugate if $\operatorname{Iso}_{\mathcal{F}}\left(P, P^{\prime}\right) \neq \emptyset$.

The fusion systems we consider here will all satisfy the following additional condition. Here, and throughout the rest of the paper, we write $\operatorname{Syl}_{p}(G)$ for the set of Sylow $p$-subgroups of $G$. Also, for any $P \leq G$ and any $g \in N_{G}(P), c_{g} \in \operatorname{Aut}(P)$ denotes the automorphism $c_{g}(x)=g x g^{-1}$.

Definition 1.2. Let $\mathcal{F}$ be a fusion system over a $p$-group $S$.

- A subgroup $P \leq S$ is fully centralized in $\mathcal{F}$ if $\left|C_{S}(P)\right| \geq\left|C_{S}\left(P^{\prime}\right)\right|$ for all $P^{\prime} \leq S$ that are $\mathcal{F}$-conjugate to $P$.

- A subgroup $P \leq S$ is fully normalized in $\mathcal{F}$ if $\left|N_{S}(P)\right| \geq\left|N_{S}\left(P^{\prime}\right)\right|$ for all $P^{\prime} \leq S$ that are $\mathcal{F}$-conjugate to $P$.

- $\mathcal{F}$ is a saturated fusion system if the following two conditions hold:

(I) Any $P \leq S$ which is fully normalized in $\mathcal{F}$ is fully centralized in $\mathcal{F}$, and $\operatorname{Aut}_{S}(P) \in \operatorname{Syl}_{p}\left(\operatorname{Aut}_{\mathcal{F}}(P)\right)$.

(II) If $P \leq S$ and $\varphi \in \operatorname{Hom}_{\mathcal{F}}(P, S)$ are such that $\varphi P$ is fully centralized, and if we set

$$
N_{\varphi}=\left\{g \in N_{S}(P) \mid \varphi c_{g} \varphi^{-1} \in \operatorname{Aut}_{S}(\varphi P)\right\},
$$

then there is $\bar{\varphi} \in \operatorname{Hom}_{\mathcal{F}}\left(N_{\varphi}, S\right)$ such that $\left.\bar{\varphi}\right|_{P}=\varphi$.

The above definition is slightly different from the definition of a "full Frobenius system" as formulated by Lluís Puig [Pu2, §2.5], but is equivalent to his definition by the remarks after Proposition A.2. Condition (I) can be thought of as a "Sylow condition". It says that $\operatorname{Out}_{\mathcal{F}}(S)$ has order prime to $p$ (just as $N_{G}(S) / S$ has order prime to $p$ if $S \in \operatorname{Syl}_{p}(G)$ ); and more generally it reflects the fact that for any $p$-subgroup $P \leq G$, there is some $S \in \operatorname{Syl}_{p}(G)$ such that $N_{S}(P) \in \operatorname{Syl}_{p}\left(N_{G}(P)\right)$. Another way of interpreting this condition is that if $\left|N_{S}(P)\right| \geq\left|N_{S}\left(P^{\prime}\right)\right|$ for $P^{\prime} \mathcal{F}$ conjugate to $P$, then $C_{S}(P)$ and $\operatorname{Aut}_{S}(P) \cong N_{S}(P) / C_{S}(P)$ must also be maximal in the same sense. As for condition (II), it is natural to require that some extension property hold for morphisms in $\mathcal{F}$, and $N_{\varphi}$ is by definition the largest subgroup of $N_{S}(P)$ to which $\varphi$ could possibly extend.

The motivating example for this definition is the fusion system of a finite group $G$. For any $S \in \operatorname{Syl}_{p}(G)$, we let $\mathcal{F}_{S}(G)$ be the fusion system over $S$ defined by setting $\operatorname{Hom}_{\mathcal{F}_{S}(G)}(P, Q)=\operatorname{Hom}_{G}(P, Q)$ for all $P, Q \leq S$.

Proposition 1.3. Let $G$ be a finite group, and let $S$ be a Sylow p-subgroup of $G$. Then the fusion system $\mathcal{F}_{S}(G)$ over $S$ is saturated. Also, a subgroup $P \leq S$ is 
fully centralized in $\mathcal{F}_{S}(G)$ if and only if $C_{S}(P) \in \operatorname{Syl}_{p}\left(C_{G}(P)\right)$, while $P$ is fully normalized in $\mathcal{F}_{S}(G)$ if and only if $N_{S}(P) \in \operatorname{Syl}_{p}\left(N_{G}(P)\right)$.

Proof. Fix some $P \leq S$, and choose $g \in G$ such that $g^{-1} S g$ contains a Sylow $p$-subgroup of $N_{G}(P)$. Then $g P g^{-1} \leq S$ and $N_{g^{-1} S g}(P) \in \operatorname{Syl}_{p}\left(N_{G}(P)\right)$, and so $N_{S}\left(g P g^{-1}\right) \in \operatorname{Syl}_{p}\left(N_{G}\left(g P g^{-1}\right)\right)$. This clearly implies that $\left|N_{S}\left(g P g^{-1}\right)\right| \geq\left|N_{S}\left(P^{\prime}\right)\right|$ for all $P^{\prime} \leq S$ that are $G$-conjugate to $P$. Thus $g g^{-1}$ is fully normalized in $\mathcal{F}_{S}(G)$, and $P$ is fully normalized in $\mathcal{F}_{S}(G)$ if and only if $\left|N_{S}(P)\right|=\left|N_{S}\left(g P g^{-1}\right)\right|$, if and only if $N_{S}(P) \in \operatorname{Syl}_{p}\left(N_{G}(P)\right)$. A similar argument proves that $P$ is fully centralized in $\mathcal{F}_{S}(G)$ if and only if $C_{S}(P) \in \operatorname{Syl}_{p}\left(C_{G}(P)\right)$.

If $P$ is fully normalized in $\mathcal{F}_{S}(G)$, then since $N_{S}(P) \in \operatorname{Syl}_{p}\left(N_{G}(P)\right)$, the obvious counting argument shows that

$$
\operatorname{Aut}_{S}(P) \in \operatorname{Syl}_{p}\left(\operatorname{Aut}_{G}(P)\right) \quad \text { and } \quad C_{S}(P) \in \operatorname{Syl}_{p}\left(C_{G}(P)\right) .
$$

In particular, $P$ is fully centralized in $\mathcal{F}_{S}(G)$, and this proves condition (I) in Definition 1.2

To see condition (II), let $P \leq S$ and $g \in G$ be such that $g P g^{-1} \leq S$ and is fully centralized in $\mathcal{F}_{S}(G)$, and write $P^{\prime}=g P g^{-1}$ for short. Set

$$
N=\left\{x \in N_{S}(P) \mid c_{g} \circ c_{x} \circ c_{g}^{-1} \in \operatorname{Aut}_{S}\left(P^{\prime}\right)\right\} \quad \text { and } \quad N_{G}=N \cdot C_{G}(P) ;
$$

and similarly

$$
N^{\prime}=\left\{x \in N_{S}\left(P^{\prime}\right) \mid c_{g}^{-1} \circ c_{x} \circ c_{g} \in \operatorname{Aut}_{S}(P)\right\} \quad \text { and } \quad N_{G}^{\prime}=N^{\prime} \cdot C_{G}\left(P^{\prime}\right) .
$$

In particular, $g N_{G} g^{-1}=N_{G}^{\prime}$ ( $N$ and $N^{\prime}$ are conjugate modulo centralizers), and thus $g N g^{-1}$ and $N^{\prime}$ are two $p$-subgroups of $N_{G}^{\prime}$. Furthermore,

$$
\left[N_{G}^{\prime}: N^{\prime}\right]=\left[C_{G}\left(P^{\prime}\right): C_{S}\left(P^{\prime}\right)\right]
$$

is prime to $p$ (since $P^{\prime}$ is fully centralized), so $N^{\prime} \in \operatorname{Syl}_{p}\left(N_{G}^{\prime}\right)$. Since $C_{G}\left(P^{\prime}\right) \triangleleft$ $N_{G}^{\prime}$ has $p$-power index, all Sylow $p$-subgroups of $N_{G}^{\prime}$ are conjugate by elements of $C_{G}\left(P^{\prime}\right)$, and hence there is $h \in C_{G}\left(P^{\prime}\right)$ such that $h\left(g N g^{-1}\right) h^{-1} \leq N^{\prime}$. Thus $c_{h g} \in \operatorname{Hom}_{\mathcal{F}_{S}(G)}(N, S)$ extends $c_{g} \in \operatorname{Hom}_{\mathcal{F}_{S}(G)}(P, S)$.

Puig's original motivation for defining fusion systems came from block theory. Let $k$ be an algebraically closed field of characteristic $p \neq 0$. A block in a group ring $k[G]$ is an indecomposable 2-sided ideal which is a direct summand. Puig showed $\mathrm{Pu}$ that the Brauer pairs associated to a block $b$ (the " $b$-subpairs"), together with the inclusion and conjugacy relations defined by Alperin and Broué $\mathrm{AB}$, form a saturated fusion system over the defect group of $b$. See, for example, AB] or Alp. Chapter IV], for definitions of defect groups and Brauer pairs of blocks.

In practice, when proving that certain fusion systems are saturated, it will be convenient to replace condition (I) by a modified version of the condition, as described in the following lemma.

Lemma 1.4. Let $\mathcal{F}$ be a fusion system over a p-group $S$ which satisfies condition (II) in Definition 1.2, and also satisfies the condition

$\left(\mathrm{I}^{\prime}\right)$ Each subgroup $P \leq S$ is $\mathcal{F}$-conjugate to a fully centralized subgroup $P^{\prime} \leq S$ such that $\operatorname{Aut}_{S}\left(P^{\prime}\right) \in \operatorname{Syl}_{p}\left(\operatorname{Aut}_{\mathcal{F}}\left(P^{\prime}\right)\right)$.

Then $\mathcal{F}$ is a saturated fusion system. 
Proof. We must prove condition (I) in Definition 1.2. Assume that $P \leq S$ is fully normalized in $\mathcal{F}$. By $\left(\mathrm{I}^{\prime}\right)$, there is $P^{\prime} \leq S$ which is $\mathcal{F}$-conjugate to $P$, and such that $P^{\prime}$ is fully centralized in $\mathcal{F}$ and $\operatorname{Aut}_{S}\left(P^{\prime}\right) \in \operatorname{Syl}_{p}\left(\operatorname{Aut}_{\mathcal{F}}\left(P^{\prime}\right)\right)$. In particular,

$$
\left|C_{S}\left(P^{\prime}\right)\right| \geq\left|C_{S}(P)\right| \quad \text { and } \quad\left|\operatorname{Aut}_{S}\left(P^{\prime}\right)\right| \geq\left|\operatorname{Aut}_{S}(P)\right| .
$$

On the other hand, since $P$ is fully normalized,

$$
\left|C_{S}(P)\right| \cdot\left|\operatorname{Aut}_{S}(P)\right|=\left|N_{S}(P)\right| \geq\left|N_{S}\left(P^{\prime}\right)\right|=\left|C_{S}\left(P^{\prime}\right)\right| \cdot\left|\operatorname{Aut}_{S}\left(P^{\prime}\right)\right|,
$$

and hence the inequalities in $(1)$ are equalities. Thus $P$ is fully centralized and $\operatorname{Aut}_{S}(P) \in \operatorname{Syl}_{p}\left(\operatorname{Aut}_{\mathcal{F}}(P)\right)$. This proves $(\mathrm{I})$.

For any pair of fusion systems $\mathcal{F}_{1}$ over $S_{1}$ and $\mathcal{F}_{2}$ over $S_{2}$, let $\mathcal{F}_{1} \times \mathcal{F}_{2}$ be the obvious fusion system over $S_{1} \times S_{2}$ :

$\operatorname{Hom}_{\mathcal{F}_{1} \times \mathcal{F}_{2}}(P, Q)=\left\{\left.\left(\alpha_{1}, \alpha_{2}\right)\right|_{P} \in \operatorname{Hom}(P, Q) \mid P \leq P_{1} \times P_{2}, \alpha_{i} \in \operatorname{Hom}_{\mathcal{F}}\left(P_{i}, S_{i}\right)\right\}$ for all $P, Q \leq S_{1} \times S_{2}$. The following technical result will be needed in Section 5

Lemma 1.5. If $\mathcal{F}_{1}$ and $\mathcal{F}_{2}$ are saturated fusion systems over $S_{1}$ and $S_{2}$, respectively, then $\mathcal{F}_{1} \times \mathcal{F}_{2}$ is a saturated fusion system over $S_{1} \times S_{2}$.

Proof. For any $P \leq S_{1} \times S_{2}$, let $P_{1} \leq S_{1}$ and $P_{2} \leq S_{2}$ denote the images of $P$ under projection to the first and second factors. Thus $P \leq P_{1} \times P_{2}$, and this is the smallest product subgroup which contains $P$. Similarly, for any $P \leq S_{1} \times S_{2}$ and any $\varphi \in \operatorname{Hom}_{\mathcal{F}_{1} \times \mathcal{F}_{2}}\left(P, S_{1} \times S_{2}\right), \varphi_{1} \in \operatorname{Hom}_{\mathcal{F}_{1}}\left(P_{1}, S_{1}\right)$ and $\varphi_{2} \in \operatorname{Hom}_{\mathcal{F}_{2}}\left(P_{2}, S_{2}\right)$ denote the projections of $\varphi$.

We apply Lemma 1.4 and first check condition (I'). Fix $P \leq S_{1} \times S_{2}$; we must show that $P$ is $\mathcal{F}_{1} \times \mathcal{F}_{2}$-conjugate to a subgroup $P^{\prime}$ which is fully centralized and satisfies $\operatorname{Aut}_{S_{1} \times S_{2}}\left(P^{\prime}\right) \in \operatorname{Syl}_{p}\left(\operatorname{Aut}_{\mathcal{F}_{1} \times \mathcal{F}_{2}}\left(P^{\prime}\right)\right)$. We can assume that $P_{1}$ and $P_{2}$ are both fully normalized; otherwise replace $P$ by an appropriate subgroup in its $\mathcal{F}_{1} \times \mathcal{F}_{2}$-conjugacy class. Since $C_{S_{1} \times S_{2}}(P)=C_{S_{1}}\left(P_{1}\right) \times C_{S_{2}}\left(P_{2}\right)$, and since (by (I) applied to the saturated fusion systems $\mathcal{F}_{i}$ ) the $P_{i}$ are fully centralized, $P$ is also fully centralized. Also, by (I) again, $\operatorname{Aut}_{S_{i}}\left(P_{i}\right) \in \operatorname{Syl}_{p}\left(\operatorname{Aut}_{\mathcal{F}_{i}}\left(P_{i}\right)\right)$, and hence $\operatorname{Aut}_{S_{1} \times S_{2}}\left(P_{1} \times P_{2}\right) \in \operatorname{Syl}_{p}\left(\operatorname{Aut}_{\mathcal{F}_{1} \times \mathcal{F}_{2}}\left(P_{1} \times P_{2}\right)\right)$. Thus, if we regard $\operatorname{Aut}_{\mathcal{F}_{1} \times \mathcal{F}_{2}}(P)$ as a subgroup of $\operatorname{Aut}_{\mathcal{F}_{1} \times \mathcal{F}_{2}}\left(P_{1} \times P_{2}\right)$, there is an element $\alpha \in \operatorname{Aut}_{\mathcal{F}_{1} \times \mathcal{F}_{2}}\left(P_{1} \times P_{2}\right)$ such that $\operatorname{Aut}_{S_{1} \times S_{2}}\left(P_{1} \times P_{2}\right)$ contains a Sylow $p$-subgroup of $\alpha \operatorname{Aut}_{\mathcal{F}_{1} \times \mathcal{F}_{2}}(P) \alpha^{-1}=$ $\operatorname{Aut}_{\mathcal{F}_{1} \times \mathcal{F}_{2}}(\alpha P)$. Then

$$
\operatorname{Aut}_{S_{1} \times S_{2}}(\alpha P)=\operatorname{Aut}_{S_{1} \times S_{2}}\left(P_{1} \times P_{2}\right) \cap \operatorname{Aut}_{\mathcal{F}_{1} \times \mathcal{F}_{2}}(\alpha P) \in \operatorname{Syl}_{p}\left(\operatorname{Aut}_{\mathcal{F}_{1} \times \mathcal{F}_{2}}(\alpha P)\right) \text {, }
$$

$\alpha P$ is still fully centralized in $\mathcal{F}_{1} \times \mathcal{F}_{2}$, and this finishes the proof of $\left(\mathrm{I}^{\prime}\right)$.

To prove condition (II), fix $P \leq S_{1} \times S_{2}$ and $\varphi \in \operatorname{Hom}_{\mathcal{F}_{1} \times \mathcal{F}_{2}}\left(P, S_{1} \times S_{2}\right)$, and assume $\varphi(P)$ is fully centralized in $\mathcal{F}_{1} \times \mathcal{F}_{2}$. Since

$$
C_{S_{1} \times S_{2}}(\varphi(P))=C_{S_{1}}\left(\varphi_{1}\left(P_{1}\right)\right) \times C_{S_{2}}\left(\varphi_{2}\left(P_{2}\right)\right),
$$

we see that $\varphi_{i}\left(P_{i}\right)$ is fully centralized in $\mathcal{F}_{i}$ for $i=1,2$. Set

$$
N_{\varphi}=\left\{g \in N_{S_{1} \times S_{2}}(P) \mid \varphi c_{g} \varphi^{-1} \in \operatorname{Aut}_{S_{1} \times S_{2}}(\varphi(P))\right\} \leq S_{1} \times S_{2}
$$

and

$$
N_{\varphi_{i}}=\left\{g \in N_{S_{i}}\left(P_{i}\right) \mid \varphi_{i} c_{g} \varphi_{i}^{-1} \in \operatorname{Aut}_{S_{i}}\left(\varphi_{i}\left(P_{i}\right)\right)\right\} \leq S_{i} .
$$

Then $\varphi$ extends to $\varphi_{1} \times \varphi_{2} \in \operatorname{Hom}_{\mathcal{F}_{1} \times \mathcal{F}_{2}}\left(P_{1} \times P_{2}, S_{1} \times S_{2}\right)$ by definition of $\mathcal{F}_{1} \times \mathcal{F}_{2}$, and hence to $N_{\varphi_{1}} \times N_{\varphi_{2}}$ by condition (II) applied to the saturated fusion systems $\mathcal{F}_{1}$ and $\mathcal{F}_{2}$. So (II) holds for the fusion system $\mathcal{F}_{1} \times \mathcal{F}_{2}\left(N_{\varphi} \leq N_{\varphi_{1}} \times N_{\varphi_{2}}\right)$, and this finishes the proof that $\mathcal{F}_{1} \times \mathcal{F}_{2}$ is saturated. 
In order to help motivate the next constructions, we recall some definitions from BLO]. If $G$ is a finite group and $p$ is a prime, then a $p$-subgroup $P \leq G$ is $p$-centric if $C_{G}(P)=Z(P) \times C_{G}^{\prime}(P)$, where $C_{G}^{\prime}(P)$ has order prime to $p$. For any $P, Q \leq G$, let $N_{G}(P, Q)$ denote the transporter: the set of all $g \in G$ such that $g P g^{-1} \leq Q$. For any $S \in \operatorname{Syl}_{p}(G), \mathcal{L}_{S}^{c}(G)$ denotes the category whose objects are the $p$-centric subgroups of $S$, and where $\operatorname{Mor}_{\mathcal{L}_{S}^{c}(G)}(P, Q)=N_{G}(P, Q) / C_{G}^{\prime}(P)$. By comparison, $\operatorname{Hom}_{G}(P, Q) \cong N_{G}(P, Q) / C_{G}(P)$. Hence there is a functor from $\mathcal{L}_{S}^{c}(G)$ to $\mathcal{F}_{S}(G)$ which is the inclusion on objects, and which sends the morphism corresponding to $g \in N_{G}(P, Q)$ to $c_{g} \in \operatorname{Hom}_{G}(P, Q)$.

Definition 1.6. Let $\mathcal{F}$ be any fusion system over a $p$-group $S$. A subgroup $P \leq S$ is $\mathcal{F}$-centric if $P$ and all of its $\mathcal{F}$-conjugates contain their $S$-centralizers. Let $\mathcal{F}^{c}$ denote the full subcategory of $\mathcal{F}$ whose objects are the $\mathcal{F}$-centric subgroups of $S$.

We are now ready to define "centric linking systems" associated to a fusion system.

Definition 1.7. Let $\mathcal{F}$ be a fusion system over the $p$-group $S$. A centric linking system associated to $\mathcal{F}$ is a category $\mathcal{L}$ whose objects are the $\mathcal{F}$-centric subgroups of $S$, together with a functor

$$
\pi: \mathcal{L} \longrightarrow \mathcal{F}^{c}
$$

and "distinguished" monomorphisms $P \stackrel{\delta_{P}}{\longrightarrow} \operatorname{Aut}_{\mathcal{L}}(P)$ for each $\mathcal{F}$-centric subgroup $P \leq S$, which satisfy the following conditions.

(A) $\pi$ is the identity on objects and surjective on morphisms. More precisely, for each pair of objects $P, Q \in \mathcal{L}, Z(P)$ acts freely on $\operatorname{Mor}_{\mathcal{L}}(P, Q)$ by composition (upon identifying $Z(P)$ with $\delta_{P}(Z(P)) \leq \operatorname{Aut}_{\mathcal{L}}(P)$ ), and $\pi$ induces a bijection



(B) For each $\mathcal{F}$-centric subgroup $P \leq S$ and each $g \in P, \pi$ sends $\delta_{P}(g) \in \operatorname{Aut}_{\mathcal{L}}(P)$ to $c_{g} \in \operatorname{Aut}_{\mathcal{F}}(P)$.

(C) For each $f \in \operatorname{Mor}_{\mathcal{L}}(P, Q)$ and each $g \in P$, the following square commutes in $\mathcal{L}:$



One easily checks that for any $G$ and any $S \in \operatorname{Syl}_{p}(G), \mathcal{L}_{S}^{c}(G)$ is a centric linking system associated to the fusion system $\mathcal{F}_{S}(G)$. Condition (C) is motivated in part because it always holds in $\mathcal{L}_{S}^{c}(G)$ for any $G$. Conditions $(\mathrm{A})$ and $(\mathrm{B})$ imply that $P$ acts freely on $\operatorname{Mor}_{\mathcal{L}}(P, Q)$. Together with $(\mathrm{C})$, they imply that the $Q$-action on $\operatorname{Mor}_{\mathcal{L}}(P, Q)$ is free, and describe how it determines the action of $P$. Condition (C) was also motivated by the proof of Proposition 2.2 below, where we show that the nerve of any centric linking system is equivalent to the homotopy colimit of a certain functor.

Throughout the rest of this paper, whenever we refer to conditions (A), (B), or (C), it will mean the conditions in the above Definition 1.7 
Definition 1.8. A p-local finite group is a triple $(S, \mathcal{F}, \mathcal{L})$, where $\mathcal{F}$ is a saturated fusion system over the $p$-group $S$ and $\mathcal{L}$ is a centric linking system associated to $\mathcal{F}$. The classifying space of the $p$-local finite group $(S, \mathcal{F}, \mathcal{L})$ is the space $|\mathcal{L}|_{p}^{\wedge}$.

Thus, for any finite group $G$ and any $S \in \operatorname{Syl}_{p}(G)$, the triple $\left(S, \mathcal{F}_{S}(G), \mathcal{L}_{S}^{c}(G)\right)$ is a $p$-local finite group. Its classifying space is $\left|\mathcal{L}_{S}^{c}(G)\right|_{p}^{\wedge} \simeq\left|\mathcal{L}_{p}^{c}(G)\right|_{p}^{\wedge}$, which by [BLO, Proposition 1.1] is homotopy equivalent to $B G_{p}^{\wedge}$.

The following notation will be used when working with $p$-local finite groups. For any group $G$, let $\mathcal{B}(G)$ denote the category with one object $o_{G}$, and one morphism denoted $\check{g}$ for each $g \in G$.

Notation 1.9. Let $(S, \mathcal{F}, \mathcal{L})$ be a p-local finite group, where $\pi: \mathcal{L} \longrightarrow \mathcal{F}^{c}$ denotes the projection functor. For each $\mathcal{F}$-centric subgroup $P \leq S$, and each $g \in P$, we write

and let

$$
\widehat{g}=\delta_{P}(g) \in \operatorname{Aut}_{\mathcal{L}}(P)
$$

$$
\theta_{P}: \mathcal{B}(P) \longrightarrow \mathcal{L}
$$

denote the functor which sends the unique object $o_{P} \in \mathrm{Ob}(\mathcal{B}(P))$ to $P$ and which sends a morphism $\check{g}$ (for $g \in P$ ) to $\widehat{g}=\delta_{P}(g)$. If $f$ is any morphism in $\mathcal{L}$, we let $[f]=\pi(f)$ denote its image in $\mathcal{F}$.

The following lemma lists some easy properties of centric linking systems associated to saturated fusion systems.

Lemma 1.10. Fix a p-local finite group $(S, \mathcal{F}, \mathcal{L})$, and let $\pi: \mathcal{L} \longrightarrow \mathcal{F}^{c}$ be the projection. Fix $\mathcal{F}$-centric subgroups $P, Q, R$ in $S$. Then the following hold.

(a) Fix any sequence $P \stackrel{\varphi}{\longrightarrow} Q \stackrel{\psi}{\longrightarrow} R$ of morphisms in $\mathcal{F}^{c}$, and let $\widetilde{\psi} \in \pi_{Q, R}^{-1}(\psi)$ and $\widetilde{\psi \varphi} \in \pi_{P, R}^{-1}(\psi \varphi)$ be arbitrary liftings. Then there is a unique morphism $\widetilde{\varphi} \in \operatorname{Mor}_{\mathcal{L}}(P, Q)$ such that

$$
\widetilde{\psi} \circ \widetilde{\varphi}=\widetilde{\psi \varphi}
$$

and furthermore $\pi_{P, Q}(\widetilde{\varphi})=\varphi$.

(b) If $\widetilde{\varphi}, \widetilde{\varphi}^{\prime} \in \operatorname{Mor}_{\mathcal{L}}(P, Q)$ are such that the homomorphisms $\varphi \stackrel{\text { def }}{=} \pi_{P, Q}(\widetilde{\varphi})$ and $\varphi^{\prime} \stackrel{\text { def }}{=} \pi_{P, Q}\left(\widetilde{\varphi}^{\prime}\right)$ are conjugate (differ by an element of $\operatorname{Inn}(Q)$ ), then there is a unique element $g \in Q$ such that $\widetilde{\varphi}^{\prime}=\widehat{g} \circ \widetilde{\varphi}$ in $\operatorname{Mor}_{\mathcal{L}}(P, Q)$.

Proof. (a) Fix any element $\alpha \in \pi_{P, Q}^{-1}(\varphi)$. By (A), there is a unique element $g \in Z(P)$ such that $\widetilde{\psi \varphi}=\widetilde{\psi} \circ \alpha \circ \widehat{g}$. Hence equation (1) holds if we set $\widetilde{\varphi}=\alpha \circ \widehat{g}$, and clearly $\pi_{P, Q}(\widetilde{\varphi})=\varphi$. Conversely, for any $\widetilde{\varphi}^{\prime} \in \operatorname{Mor}_{\mathcal{L}}(P, Q)$ such that $\widetilde{\psi} \circ \widetilde{\varphi}^{\prime}=\widetilde{\psi \varphi}$ we have $\pi_{P, Q}\left(\widetilde{\varphi}^{\prime}\right)=\varphi$ since they are equal after composing with $\psi$, and so $\widetilde{\varphi}^{\prime}=\widetilde{\varphi}$ since (by (A) again) the same group $Z(P)$ acts freely and transitively on $\pi_{P, Q}^{-1}(\varphi)$ and on $\pi_{P, R}^{-1}(\psi \varphi)$.

(b) If $x \in Q$ is such that

$$
\pi_{P, Q}\left(\widetilde{\varphi}^{\prime}\right)=\varphi^{\prime}=c_{x} \circ \varphi=\pi_{P, Q}(\widehat{x} \circ \widetilde{\varphi}),
$$

then by $(\mathrm{A})$ and $(\mathrm{C})$, there is a unique element $y \in Z(P)$ such that

$$
\widetilde{\varphi}^{\prime}=\widehat{x} \circ \widetilde{\varphi} \circ \widehat{y}=\widehat{x} \circ \widehat{\varphi(y)} \circ \widetilde{\varphi} .
$$


This proves the existence of $g=x \cdot \varphi(y) \in Q$ such that $\widetilde{\varphi}^{\prime}=\widehat{g} \circ \widetilde{\varphi}$. Conversely, if

$$
\widetilde{\varphi}^{\prime}=\widehat{g} \circ \widetilde{\varphi}=\widehat{h} \circ \widetilde{\varphi}
$$

for $g, h \in Q$, then $\widetilde{\varphi}=\widehat{g^{-1} h} \circ \widetilde{\varphi}$, so $g^{-1} h \in C_{Q}(\varphi(P))$, and $g^{-1} h \in \varphi(P)$ since $P$ (and hence $\varphi(P))$ is $\mathcal{F}$-centric. Write $g^{-1} h=\varphi(y)$ for $y \in P$; then $\widetilde{\varphi}=\widetilde{\varphi} \circ \widehat{y}$ by (C), hence $\widehat{y}=$ Id by (a), and so $y=1$ (and $g=h$ ) by (A).

Lemma 1.10(a) implies in particular that all morphisms in $\mathcal{L}$ are monomorphisms in the categorical sense.

The next proposition describes how an associated centric linking system $\mathcal{L}$ over a $p$-group $S$ contains the category with the same objects and whose morphisms are the sets $N_{S}(P, Q)$.

Proposition 1.11. Let $(S, \mathcal{F}, \mathcal{L})$ be a p-local finite group, and let $\pi: \mathcal{L} \longrightarrow \mathcal{F}^{c}$ be the associated projection. For each $P \leq S$, fix a choice of "inclusion" morphism $\iota_{P} \in \operatorname{Mor}_{\mathcal{L}}(P, S)$ such that $\left[\iota_{P}\right]=\operatorname{incl} \in \operatorname{Hom}(P, S)$ (and $\left.\iota_{S}=\operatorname{Id}_{S}\right)$. Then there are unique injections

$$
\delta_{P, Q}: N_{S}(P, Q) \longrightarrow \operatorname{Mor}_{\mathcal{L}}(P, Q),
$$

defined for all $\mathcal{F}$-centric subgroups $P, Q \leq S$, which have the following properties.

(a) For all $\mathcal{F}$-centric $P, Q \leq S$ and all $g \in N_{S}(P, Q),\left[\delta_{P, Q}(g)\right]=c_{g} \in \operatorname{Hom}_{\mathcal{F}}(P, Q)$.

(b) For all $\mathcal{F}$-centric $P \leq S$ we have $\delta_{P, S}(1)=\iota_{P}$, and $\delta_{P, P}(g)=\delta_{P}(g)$ for $g \in P$.

(c) For all $\mathcal{F}$-centric $P, Q, R \leq S$ and all $g \in N_{S}(P, Q)$ and $h \in N_{S}(Q, R)$ we have $\delta_{Q, R}(h) \circ \delta_{P, Q}(g)=\delta_{P, R}(h g)$.

Proof. For each $\mathcal{F}$-centric $P$ and $Q$ and each $g \in N_{S}(P, Q)$, there is by Lemma 1.10(a) a unique morphism $\delta_{P, Q}(g) \in \operatorname{Mor}_{\mathcal{L}}(P, Q)$ such that $\left[\delta_{P, Q}(g)\right]=c_{g}$, and such that the following square commutes:

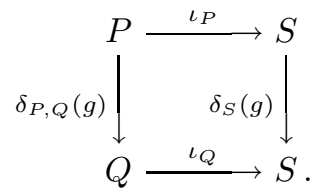

Conditions (b) and (c) above also follow from the uniqueness property in Lemma 1.10(a). The injectivity of $\delta_{P, Q}$ follows from condition (A), since $\left[\delta_{P, Q}(g)\right]=$ $\left[\delta_{P, Q}(h)\right]$ in $\operatorname{Hom}_{\mathcal{F}}(P, Q)$ if and only if $h^{-1} g \in C_{S}(P)=Z(P)$.

We finish the section with the following proposition, which shows that the classifying space of any $p$-local finite group is $p$-complete, and also provides some control over its fundamental group.

Proposition 1.12. Let $(S, \mathcal{F}, \mathcal{L})$ be any p-local finite group. Then $|\mathcal{L}|$ is $p$-good. Also, the composite

$$
S \stackrel{\pi_{1}\left(\left|\theta_{S}\right|\right)}{\longrightarrow} \pi_{1}(|\mathcal{L}|) \longrightarrow \pi_{1}\left(|\mathcal{L}|_{p}^{\wedge}\right),
$$

induced by the inclusion $\mathcal{B}(S) \stackrel{\theta_{S}}{\longrightarrow} \mathcal{L}$, is surjective.

Proof. For each $\mathcal{F}$-centric subgroup $P \leq S$, fix a morphism $\iota_{P} \in \operatorname{Mor}_{\mathcal{L}}(P, S)$ which lifts the inclusion (and set $\iota_{S}=\operatorname{Id}_{S}$ ). By Lemma 1.10(a), for each $P \leq Q \leq S$, there is a unique morphism $\iota_{P}^{Q} \in \operatorname{Mor}_{\mathcal{L}}(P, Q)$ such that $\iota_{Q} \circ \iota_{P}^{Q}=\iota_{P}$. 
Regard the vertex $S$ as the basepoint of $|\mathcal{L}|$. Define

$$
\omega: \operatorname{Mor}(\mathcal{L}) \longrightarrow \pi_{1}(|\mathcal{L}|)
$$

by sending each $\varphi \in \operatorname{Mor}_{\mathcal{L}}(P, Q)$ to the loop formed by the edges $\iota_{P}, \varphi$, and $\iota_{Q}$ (in that order). Clearly, $\omega(\psi \circ \varphi)=\omega(\psi) \cdot \omega(\varphi)$ whenever $\psi$ and $\varphi$ are composable, and $\omega\left(\iota_{P}^{Q}\right)=\omega\left(\iota_{P}\right)=1$ for all $P \leq Q \leq S$. Also, $\pi_{1}(|\mathcal{L}|)$ is generated by $\operatorname{Im}(\omega)$, since any loop in $|\mathcal{L}|$ can be split up as a composite of loops of the above form.

By Alperin's fusion theorem for saturated fusion systems (Theorem A.10), each morphism in $\mathcal{F}$, and hence each morphism in $\mathcal{L}$, is (up to inclusions) a composite of automorphisms of fully normalized $\mathcal{F}$-centric subgroups. Thus $\pi_{1}(|\mathcal{L}|)$ is generated by the subgroups $\omega\left(\operatorname{Aut}_{\mathcal{L}}(P)\right)$ for all fully normalized $\mathcal{F}$-centric $P \leq S$.

Let $K \triangleleft \pi_{1}(|\mathcal{L}|)$ be the subgroup generated by all elements of finite order prime to $p$. For each fully normalized $\mathcal{F}$-centric $P \leq S$, $\operatorname{Aut}_{\mathcal{L}}(P)$ is generated by its Sylow subgroup $N_{S}(P)$ together with elements of order prime to $p$. Hence $\pi_{1}(|\mathcal{L}|)$ is generated by $K$ together with the subgroups $\omega\left(N_{S}(P)\right)$; and $\omega\left(N_{S}(P)\right) \leq \omega(S)$ for each $P$. This shows that $\omega$ sends $S$ surjectively onto $\pi_{1}(|\mathcal{L}|) / K$, and in particular that this quotient group is a finite $p$-group.

Set $\pi=\pi_{1}(|\mathcal{L}|) / K$ for short. Since $K$ is generated by elements of order prime to $p$, the same is true of its abelianization, and hence $H_{1}\left(K ; \mathbb{F}_{p}\right)=0$. Thus, $K$ is $p$ perfect. Let $X$ be the cover of $|\mathcal{L}|$ with fundamental group $K$. Then $X$ is $p$-good and $X_{p}^{\wedge}$ is simply connected since $\pi_{1}(X)$ is $p$-perfect [BK, VII.3.2]. Also, $H_{i}\left(X ; \mathbb{F}_{p}\right)$ is finite for all $i$ since $|\mathcal{L}|$ and hence $X$ has finite skeleta. Hence $X_{p}^{\wedge} \longrightarrow|\mathcal{L}|_{p}^{\wedge} \longrightarrow B \pi$ is a fibration sequence and $|\mathcal{L}|_{p}^{\wedge}$ is $p$-complete by $[\mathrm{BK}$, II.5.2(iv)]. So $|\mathcal{L}|$ is $p$-good, and $\pi_{1}\left(|\mathcal{L}|_{p}^{\wedge}\right) \cong \pi$ is a quotient group of $S$. (Alternatively, this follows directly from a "mod $p$ plus construction" on $|\mathcal{L}|$ : there are a space $Y$ and a $\bmod p$ homology equivalence $|\mathcal{L}| \longrightarrow Y$ such that $\pi_{1}(Y) \cong \pi_{1}(|\mathcal{L}|) / K=\pi$, and $|\mathcal{L}|$ is $p$-good since $Y$ is.)

\section{Homotopy DECOMPOSITIONS OF CLASSIFYING SPACES}

We now consider some homotopy decompositions of the classifying space $|\mathcal{L}|_{p}^{\wedge}$ of a $p$-local finite group $(S, \mathcal{F}, \mathcal{L})$. The first, and most important, is taken over the orbit category of $\mathcal{F}$.

Definition 2.1. The orbit category of a fusion system $\mathcal{F}$ over a $p$-group $S$ is the category $\mathcal{O}(\mathcal{F})$ whose objects are the subgroups of $S$, and whose morphisms are defined by

$$
\operatorname{Mor}_{\mathcal{O}(\mathcal{F})}(P, Q)=\operatorname{Rep}_{\mathcal{F}}(P, Q) \stackrel{\text { def }}{=} \operatorname{Inn}(Q) \backslash \operatorname{Hom}_{\mathcal{F}}(P, Q) .
$$

We let $\mathcal{O}^{c}(\mathcal{F})$ denote the full subcategory of $\mathcal{O}(\mathcal{F})$ whose objects are the $\mathcal{F}$-centric subgroups of $S$. If $\mathcal{L}$ is a centric linking system associated to $\mathcal{F}$, then $\tilde{\pi}$ denotes the composite functor

$$
\tilde{\pi}: \mathcal{L} \stackrel{\pi}{\longrightarrow} \mathcal{F}^{c} \longrightarrow \mathcal{O}^{c}(\mathcal{F}) .
$$

More generally, if $\mathcal{F}_{0} \subseteq \mathcal{F}$ is any full subcategory, then $\mathcal{O}\left(\mathcal{F}_{0}\right)$ denotes the full subcategory of $\mathcal{O}(\mathcal{F})$ whose objects are the objects of $\mathcal{F}_{0}$. Thus, $\mathcal{O}^{c}(\mathcal{F})=\mathcal{O}\left(\mathcal{F}^{c}\right)$.

Note the difference between the orbit category of a fusion system and the orbit category of a group. If $G$ is a group and $S \in \operatorname{Syl}_{p}(G)$, then $\mathcal{O}_{S}(G)$ is the category whose objects are the orbits $G / P$ for all $P \leq S$, and where $\operatorname{Mor}_{\mathcal{O}_{S}(G)}(G / P, G / Q)$ is the set of all $G$-maps between the orbits. If $\mathcal{F}=\mathcal{F}_{S}(G)$ is the fusion system of 
$G$, then morphisms in the orbit categories of $G$ and $\mathcal{F}$ can be expressed in terms of the set $N_{G}(P, Q)$ of elements which conjugate $P$ into $Q$ :

$$
\operatorname{Mor}_{\mathcal{O}_{S}(G)}(G / P, G / Q) \cong Q \backslash N_{G}(P, Q)
$$

while

$$
\operatorname{Mor}_{\mathcal{O}(\mathcal{F})}(P, Q) \cong Q \backslash N_{G}(P, Q) / C_{G}(P) .
$$

If $P$ is $p$-centric in $G$, then these sets differ only by the action of the group $C_{G}^{\prime}(P)$ of order prime to $p$.

We next look at the homotopy type of the nerve of a centric linking system. Here, Top denotes the category of spaces.

Proposition 2.2. Fix a saturated fusion system $\mathcal{F}$ and an associated centric linking system $\mathcal{L}$, and let $\widetilde{\pi}: \mathcal{L} \longrightarrow \mathcal{O}^{c}(\mathcal{F})$ be the projection functor. Let

$$
\widetilde{B}: \mathcal{O}^{c}(\mathcal{F}) \longrightarrow \text { Top }
$$

be the left homotopy Kan extension over $\tilde{\pi}$ of the constant functor $\mathcal{L} \stackrel{*}{\longrightarrow}$ Top. Then $\widetilde{B}$ is a homotopy lifting of the homotopy functor $P \mapsto B P$, and

$$
|\mathcal{L}| \simeq \underset{\mathcal{O}^{c}(\mathcal{F})}{\operatorname{hocolim}}(\widetilde{B}) .
$$

More generally, if $\mathcal{L}_{0} \subseteq \mathcal{L}$ is any full subcategory, and $\mathcal{F}_{0} \subseteq \mathcal{F}^{c}$ is the full subcategory with $\mathrm{Ob}\left(\mathcal{F}_{0}\right)=\mathrm{Ob}\left(\mathcal{L}_{0}\right)$, then

$$
\left|\mathcal{L}_{0}\right| \simeq \underset{\mathcal{O}\left(\mathcal{F}_{0}\right)}{\operatorname{hocolim}}(\widetilde{B}) .
$$

Proof. Recall that we write $\operatorname{Rep}_{\mathcal{F}}(P, Q)$ to denote morphisms in $\mathcal{O}^{c}(\mathcal{F})$. By definition, for each $\mathcal{F}$-centric subgroup $P \leq S, \widetilde{B}(P)$ is the nerve (homotopy colimit of the point functor) of the overcategory $\widetilde{\pi} \downarrow P$, whose objects are pairs $(Q, \alpha)$ for $\alpha \in \operatorname{Rep}_{\mathcal{F}}(Q, P)$, and where

$$
\operatorname{Mor}_{\tilde{\pi} \downarrow P}((Q, \alpha),(R, \beta))=\left\{\varphi \in \operatorname{Mor}_{\mathcal{L}}(Q, R) \mid \alpha=\beta \circ \widetilde{\pi}_{Q, R}(\varphi)\right\} .
$$

Since $|\mathcal{L}| \cong \operatorname{hocolim}_{\mathcal{L}}(*)$, (1) holds by [HV] Theorem 5.5] (and the basic idea is due to Segal [Se, Proposition B.1]). Similarly, if $\widetilde{B}_{0}$ denotes the left homotopy Kan extension over $\mathcal{L}_{0} \stackrel{\widetilde{\pi}_{0}}{\longrightarrow} \mathcal{O}\left(\mathcal{F}_{0}\right)$ of the constant functor $\mathcal{L}_{0} \stackrel{*}{\longrightarrow}$ Top, then

$$
\left|\mathcal{L}_{0}\right| \simeq \underset{\mathcal{O}\left(\mathcal{F}_{0}\right)}{\operatorname{hocolim}}\left(\widetilde{B}_{0}\right)
$$

It remains only to show that $\widetilde{B}$ is a lifting of the homotopy functor $P \mapsto B P$, and that the inclusion $\widetilde{B}_{0}(P) \hookrightarrow \widetilde{B}(P)$ is a homotopy equivalence when $P \in \operatorname{Ob}\left(\mathcal{F}_{0}\right)$.

Let $\mathcal{B}^{\prime}(P) \subseteq \widetilde{\pi} \downarrow P$ be the subcategory with one object $(P$, Id) and with morphisms $\{\widehat{g} \mid g \in P\}$. In particular, $\left|\mathcal{B}^{\prime}(P)\right| \simeq B P$. We claim that $\left|\mathcal{B}^{\prime}(P)\right|$ is a deformation retract of $|\widetilde{\pi} \downarrow P|$. To see this, we must define a functor $\Psi: \widetilde{\pi} \downarrow P \longrightarrow \mathcal{B}^{\prime}(P)$ such that $\left.\Psi\right|_{\mathcal{B}^{\prime}(P)}=\mathrm{Id}$, together with a natural transformation $f:$ Id $\longrightarrow \operatorname{incl} \circ \Psi$ of functors from $\tilde{\pi} \downarrow P$ to itself. Fix a section $\widetilde{\sigma}: \operatorname{Mor}\left(\mathcal{O}^{c}(\mathcal{F})\right) \longrightarrow \operatorname{Mor}(\mathcal{L})$ of $\tilde{\pi}$ which sends identity morphisms to identity morphisms. To define $\Psi$, send each object to the unique object $(P, \mathrm{Id})$ of $\mathcal{B}^{\prime}(P)$, and send $\varphi \in \operatorname{Mor}_{\tilde{\pi} \downarrow}((Q, \alpha),(R, \beta))$ to the 
unique map $\widehat{g}=\Psi(\varphi)$ (for $g \in P$, see Lemma 1.10(b)) such that the following square commutes:



Finally, define $f:$ Id $\longrightarrow \operatorname{incl} \circ \Psi$ by sending each object $(Q, \alpha)$ to the morphism $\widetilde{\sigma}(\alpha) \in \operatorname{Mor}_{\mathcal{L}}(Q, P)$. This is clearly a natural transformation of functors, and thus

$$
\widetilde{B}(P)=|\widetilde{\pi} \downarrow P| \simeq\left|\mathcal{B}^{\prime}(P)\right| \simeq B P .
$$

If in addition $P \in \operatorname{Ob}\left(\mathcal{F}_{0}\right)$, then this restricts to a deformation retraction of $\widetilde{B}_{0}(P)=$ $\left|\widetilde{\pi}_{0} \downarrow P\right|$ to $\left|\mathcal{B}^{\prime}(P)\right|$.

To finish the proof that $\widetilde{B}$ is a lifting of the homotopy functor $P \mapsto B P$, we must show, for any $\varphi \in \operatorname{Hom}_{\mathcal{F}}(P, Q)$, that the following square commutes up to natural equivalence:

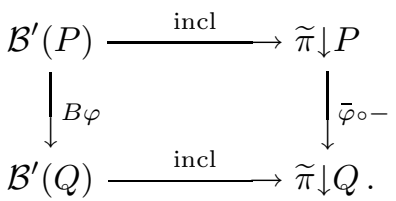

Here, $\bar{\varphi} \in \operatorname{Rep}_{\mathcal{F}}(P, Q)$ denotes the class of $\varphi$. This means constructing a natural transformation $F_{1} \stackrel{\Phi}{\longrightarrow} F_{2}$ of functors $\mathcal{B}^{\prime}(P) \longrightarrow \widetilde{\pi} \downarrow Q$, where $F_{1}=(\bar{\varphi} \circ-) \circ$ incl and $F_{2}=\operatorname{incl} \circ B \varphi$ are given by the formulas

$$
F_{1}(P, \operatorname{Id})=(P, \bar{\varphi}), \quad F_{1}(\widehat{g})=\widehat{g}, \quad \text { and } \quad F_{2}(P, \mathrm{Id})=(Q, \mathrm{Id}), \quad F_{2}(\widehat{g})=\widehat{\varphi g} .
$$

Let $\widetilde{\varphi} \in \operatorname{Mor}_{\mathcal{L}}(P, Q)$ be any lifting of $\varphi$. Then by condition $(\mathrm{C}), \Phi$ can be defined by sending the object $(P, \mathrm{Id})$ to the morphism $\widetilde{\varphi} \in \operatorname{Mor}_{\tilde{\pi} \downarrow Q}((P, \bar{\varphi}),(Q, \mathrm{Id}))$.

We will see in the next section that the obstruction groups to the existence and uniqueness of associated centric linking systems (Proposition 3.1) are exactly the same as the obstruction groups of Dwyer and Kan [DK2 to the existence and uniqueness of liftings of the homotopy functor $P \mapsto B P$. So it is not surprising that there should be a correspondence between the two. This connection is described in more detail in the next proposition, and in remarks which follow its proof.

Proposition 2.3. A saturated fusion system $\mathcal{F}$ has an associated centric linking system if and only if the homotopy functor $P \mapsto B P$ on $\mathcal{O}^{c}(\mathcal{F})$ lifts to Top.

Proof. If $\mathcal{F}$ has an associated centric linking system, then by Proposition 2.2, the homotopy functor $P \mapsto B P$ lifts to a functor

$$
\widetilde{B}: \mathcal{O}^{c}(\mathcal{F}) \longrightarrow \text { Top }
$$

defined by left homotopy Kan extension. So it remains to prove the converse.

We first fix some notation. For any space $X$ and any $x, x^{\prime} \in X, \pi_{1}(X, x)$ denotes as usual the fundamental group of $X$ based at $x$, and by extension $\pi_{1}\left(X ; x, x^{\prime}\right)$ denotes the set of homotopy classes of paths in $X$ (relative to endpoints) from $x$ to $x^{\prime}$. For any $u \in \pi_{1}\left(X ; x, x^{\prime}\right), u_{*}$ denotes the induced isomorphism from $\pi_{1}(X, x)$ to $\pi_{1}\left(X, x^{\prime}\right)$. Also, for any map of spaces $f: X \rightarrow Y, f_{*}$ denotes the induced map from $\pi_{1}\left(X ; x, x^{\prime}\right)$ to $\pi_{1}\left(Y ; f(x), f\left(x^{\prime}\right)\right)$. 
Fix a homotopy lifting $\widetilde{B}: \mathcal{O}^{c}(\mathcal{F}) \longrightarrow$ Top. Thus, $\widetilde{B}$ is a functor, equipped with homotopy classes of homotopy equivalences $\eta_{P} \in[B P, \widetilde{B}(P)]$, such that the following square in hoTop commutes for each $\varphi \in \operatorname{Mor}_{\mathcal{O}^{c}(\mathcal{F})}(P, Q)=\operatorname{Rep}_{\mathcal{F}}(P, Q)$ :

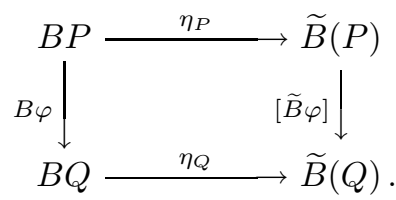

For each $P$ in $\mathcal{F}^{c}$, choose a map $\widehat{\eta}_{P}$ in the homotopy class of $\eta_{P}$, let $*_{P} \in \widetilde{B}(P)$ be the image under $\widehat{\eta}_{P}$ of the base point of $B P$, and let

$$
\gamma_{P}: P \stackrel{\cong}{\longrightarrow} \pi_{1}\left(\widetilde{B}(P), *_{P}\right)
$$

be the isomorphism induced by $\widehat{\eta}_{P}$ on fundamental groups.

Let $\mathcal{L}$ be the category whose objects are the $\mathcal{F}$-centric subgroups of $S$, and where

$$
\operatorname{Mor}_{\mathcal{L}}(P, Q)=\left\{(\varphi, u) \mid \varphi \in \operatorname{Rep}_{\mathcal{F}}(P, Q), u \in \pi_{1}\left(\widetilde{B}(Q) ; \widetilde{B} \varphi\left(*_{P}\right), *_{Q}\right)\right\} .
$$

Composition is defined by setting

$$
(\psi, v) \circ(\varphi, u)=\left(\psi \varphi, v \cdot \widetilde{B} \psi_{*}(u)\right)
$$

where paths are composed from right to left. Let $\pi: \mathcal{L} \longrightarrow \mathcal{F}^{c}$ be the functor which is the identity on objects, and where $\pi_{P, Q}$ sends $(\varphi, u) \in \operatorname{Mor}_{\mathcal{L}}(P, Q)$ to the homomorphism

$$
P \stackrel{\gamma_{P}}{\cong} \pi_{1}\left(\widetilde{B}(P), *_{P}\right) \stackrel{\varphi_{*}}{\longrightarrow} \pi_{1}\left(\widetilde{B}(Q), \widetilde{B} \varphi\left(*_{P}\right)\right) \stackrel{u_{*}}{\longrightarrow} \pi_{1}\left(\widetilde{B}(Q), *_{Q}\right) \stackrel{\gamma_{Q}^{-1}}{\cong} Q .
$$

Finally, for each $P$, define

$$
\delta_{P}: P \longrightarrow \operatorname{Aut}_{\mathcal{L}}(P) \quad \text { by setting } \quad \delta_{P}(g)=\left(\operatorname{Id}_{P}, \gamma_{P}(g)\right) .
$$

These structures are easily seen to satisfy all of the axioms in Definition 1.7 and hence define a centric linking system associated to $\mathcal{F}$.

In fact, if one uses the obvious equivalence relation between homotopy liftings (as defined in [DK] and [DK2]), then there is a one-to-one correspondence between the set of isomorphism classes of centric linking systems associated to $\mathcal{F}$ and the set of equivalence classes of homotopy liftings of the functor $P \mapsto B P$. More precisely, consider the maps

$$
\left\{\begin{array}{c}
\text { centric linking systems } \\
\text { associated to } \mathcal{F} \\
\text { up to isomorphism }
\end{array}\right\} \stackrel{K}{\rightleftarrows}\left\{\begin{array}{c}
\text { homotopy liftings of } \\
\mathcal{O}^{c}(\mathcal{F}) \stackrel{B}{\longrightarrow} \text { hoTop } \\
\text { up to equivalence }
\end{array}\right\}
$$

where $K$ is defined by left homotopy Kan extension as in Proposition 2.2 and $L$ is defined as in the proof of Proposition 2.3. Then these are both well defined, and can be shown to be inverses to each other.

In the rest of this section, we present a second decomposition of $|\mathcal{L}|$ as a homotopy colimit, analogous to the centralizer decomposition of $B G$ of Jackowski and McClure [JM], and to the centralizer decomposition of certain algebras due to Dwyer and Wilkerson [DW1]. This new decomposition will be important later on when computing $H^{*}\left(|\mathcal{L}|_{p}^{\wedge}\right)$.

Recall that for a saturated fusion system $\mathcal{F}$ over $S$, a subgroup $Q \leq S$ is fully centralized in $\mathcal{F}$ if $\left|C_{S}(Q)\right|$ is maximal among the $\left|C_{S}\left(Q^{\prime}\right)\right|$ for $Q^{\prime} \mathcal{F}$-conjugate to 
$Q$ (Definition 1.2). For any such $Q, C_{\mathcal{F}}(Q)$ is the fusion system over $C_{S}(Q)$ defined by setting

$$
\begin{aligned}
& \operatorname{Hom}_{C_{\mathcal{F}}(Q)}\left(P, P^{\prime}\right) \\
& \quad=\left\{\varphi \in \operatorname{Hom}_{\mathcal{F}}\left(P, P^{\prime}\right)\left|\exists \bar{\varphi} \in \operatorname{Hom}_{\mathcal{F}}\left(P Q, P^{\prime} Q\right), \bar{\varphi}\right|_{P}=\varphi,\left.\bar{\varphi}\right|_{Q}=\operatorname{Id}_{Q}\right\}
\end{aligned}
$$

for all $P, P^{\prime} \leq C_{S}(Q)$ (see Definition A.3). We next construct a centric linking system associated to $C_{\mathcal{F}}(Q)$.

Definition 2.4. Fix a $p$-local finite group $(S, \mathcal{F}, \mathcal{L})$, and a subgroup $Q \leq S$ which is fully centralized in $\mathcal{F}$. Define $C_{\mathcal{L}}(Q)$ to be the category whose objects are the $C_{\mathcal{F}}(Q)$-centric subgroups $P \leq C_{S}(Q)$, and where $\operatorname{Mor}_{C_{\mathcal{L}}(Q)}\left(P, P^{\prime}\right)$ is the set of those morphisms $\varphi \in \operatorname{Mor}_{\mathcal{L}}\left(P Q, P^{\prime} Q\right)$ whose underlying homomorphisms are the identity on $Q$ and send $P$ into $P^{\prime}$.

We will need the following properties of these categories. Recall that if $\mathcal{F}$ is a fusion system over $S$, then a subgroup $P \leq S$ is $\mathcal{F}$-centric if $C_{S}\left(P^{\prime}\right)=Z\left(P^{\prime}\right)$ (equivalently $C_{S}\left(P^{\prime}\right) \leq P^{\prime}$ ) for all $P^{\prime}$ that are $\mathcal{F}$-conjugate to $P$.

Proposition 2.5. Fix a saturated fusion system $\mathcal{F}$ over a p-group $S$, and a subgroup $Q \leq S$ which is fully centralized in $\mathcal{F}$. Then the following hold:

(a) A subgroup $P \leq C_{S}(Q)$ is $C_{\mathcal{F}}(Q)$-centric if and only if $P \geq Z(Q)$ and $P Q$ is $\mathcal{F}$-centric; and if this holds then $Z(P)=Z(P Q)$.

(b) $C_{\mathcal{F}}(Q)$ is a saturated fusion system over $C_{S}(Q)$.

(c) If $\mathcal{L}$ is a centric linking system associated to $\mathcal{F}$, then $C_{\mathcal{L}}(Q)$ is a centric linking system associated to $C_{\mathcal{F}}(Q)$.

Proof. We first check point (a). Fix $P \leq C_{S}(Q)$. If $P Q$ is $\mathcal{F}$-centric and $P \geq Z(Q)$, then

$$
C_{C_{S}(Q)}(P)=C_{S}(P) \cap C_{S}(Q)=C_{S}(P Q)=Z(P Q)=Z(P) \cdot Z(Q)=Z(P):
$$

the last two steps since $[P, Q]=1$ and $P \geq Z(Q)$. The same computation applies to any $P^{\prime}$ which is $C_{\mathcal{F}}(Q)$-conjugate to $P$, and so $P$ is $C_{\mathcal{F}}(Q)$-centric in this case. Conversely, if $P$ is $C_{\mathcal{F}}(Q)$-centric, then clearly $P \geq Z(Q)$. To see that $P Q$ is $\mathcal{F}$ centric, fix any $\varphi \in \operatorname{Hom}_{\mathcal{F}}(P Q, S)$; we must show that $C_{S}(\varphi(P Q)) \leq \varphi(P Q)$. Since $Q$ is fully centralized in $\mathcal{F}$, there is a homomorphism

$$
\psi \in \operatorname{Hom}_{\mathcal{F}}\left(C_{S}(\varphi(Q)) \cdot \varphi(Q), C_{S}(Q) \cdot Q\right)
$$

such that $\left.\psi\right|_{\varphi(Q)}=\left(\left.\varphi\right|_{Q}\right)^{-1}$. Set $\varphi^{\prime}=\psi \circ \varphi$; thus $\left.\varphi^{\prime}\right|_{Q}=\operatorname{Id}_{Q}$ and hence $\left.\varphi^{\prime}\right|_{P} \in$ $\operatorname{Hom}_{C_{\mathcal{F}}(Q)}\left(P, C_{S}(Q)\right)$. Then

$$
C_{S}\left(\varphi^{\prime}(P Q)\right)=C_{S}\left(\varphi^{\prime}(P) \cdot Q\right)=C_{C_{S}(Q)}\left(\varphi^{\prime}(P)\right) \leq \varphi^{\prime}(P) \leq \varphi^{\prime}(P Q),
$$

since $P$ is $C_{\mathcal{F}}(Q)$-centric, so $C_{S}(\varphi(P Q)) \leq \varphi(P Q)$ since $\psi$ sends $C_{S}(\varphi(P Q))$ injectively into $C_{S}\left(\varphi^{\prime}(P Q)\right)$; and thus $P Q$ is $\mathcal{F}$-centric.

Point (b) is a special case of Proposition A.6

When showing that $C_{\mathcal{L}}(Q)$ is a centric linking system associated to $C_{\mathcal{F}}(Q)$, note first that the category is well defined by (a): $P Q$ is $\mathcal{F}$-centric whenever $P$ is $C_{\mathcal{F}}(Q)$ centric. Conditions $(\mathrm{B})$ and $(\mathrm{C})$ are immediate. Condition $(\mathrm{A})$ - the requirement that $Z(P)$ act freely on $\operatorname{Aut}_{C_{\mathcal{L}}(Q)}(P)$ with orbit set $\operatorname{Aut}_{C_{\mathcal{F}}(Q)}(P)$ - follows since $Z(P)=Z(P Q)$ by $($ a) . 
Thus, for any $p$-local finite group $(S, \mathcal{F}, \mathcal{L})$ and any fully centralized subgroup $Q \leq S$, we have shown that $\left(C_{S}(Q), C_{\mathcal{F}}(Q), C_{\mathcal{L}}(Q)\right)$ is again a $p$-local finite group: the centralizer of $Q$ in $(S, \mathcal{F}, \mathcal{L})$.

With these definitions, the centralizer decomposition of $|\mathcal{L}|_{p}^{\wedge}$ is a formality.

Theorem 2.6. Fix a p-local finite group $(S, \mathcal{F}, \mathcal{L})$. Let $\mathcal{F}^{e}$ be the full subcategory of $\mathcal{F}$ whose objects are the nontrivial elementary abelian p-subgroups of $S$ which are fully centralized in $\mathcal{F}$. For each such $E$, let $\bar{C}_{\mathcal{L}}(E)$ be the category whose objects are the pairs $(P, \alpha)$ for $P$ in $\mathcal{L}$ and $\alpha \in \operatorname{Hom}_{\mathcal{F}}(E, Z(P))$, and where

$$
\operatorname{Mor}_{\bar{C}_{\mathcal{L}}(E)}((P, \alpha),(Q, \beta))=\left\{\varphi \in \operatorname{Mor}_{\mathcal{L}}(P, Q) \mid \pi(\varphi) \circ \alpha=\beta\right\} .
$$

Then the natural map

$$
\underset{E \in\left(\mathcal{F}^{e}\right)^{o p}}{\operatorname{hocolim}}\left|\bar{C}_{\mathcal{L}}(E)\right| \longrightarrow|\mathcal{L}|
$$

induced by the forgetful functors $(P, \alpha) \mapsto P$, is a homotopy equivalence. Also, for each $E$, the functor $P \mapsto(P$, incl $)$ induces a homotopy equivalence $\left|C_{\mathcal{L}}(E)\right| \longrightarrow$ $\left|\bar{C}_{\mathcal{L}}(E)\right|$.

Proof. Let $\widehat{\mathcal{L}}$ denote the category whose objects are the pairs $(P, E)$ for $\mathcal{F}$-centric subgroups $P \leq S$ and elementary abelian subgroups $E \leq Z(P)$, and where a morphism from $(P, E)$ to $\left(P^{\prime}, E^{\prime}\right)$ is a morphism $\varphi \in \operatorname{Hom}_{\mathcal{L}}\left(P, P^{\prime}\right)$ such that $[\varphi](E) \geq E^{\prime}$. For each $P \leq S$, let $\mathcal{E}(P) \leq Z(P)$ denote the subgroup of elements of order $p$ in the center. There are obvious functors

$$
\mathcal{L} \underset{T}{\stackrel{S}{\rightleftarrows}} \widehat{\mathcal{L}}
$$

defined by setting $S(P)=(P, \mathcal{E}(P))$ and $T(P, E)=P$, and a morphism of functors $S \circ T \longrightarrow \operatorname{Id}_{\widehat{\mathcal{L}}}$. This shows that $|\mathcal{L}| \simeq|\widehat{\mathcal{L}}|$.

Let $\tau: \widehat{\mathcal{L}} \longrightarrow\left(\mathcal{F}^{e}\right)^{\text {op }}$ be the functor which sends an object $(P, E)$ to $E$. Then by [HV, Theorem 5.5],

$$
|\mathcal{L}| \simeq|\widehat{\mathcal{L}}|=\underset{\widehat{\mathcal{L}}}{\operatorname{hocolim}}(*) \simeq \underset{E \in\left(\mathcal{F}^{e}\right)^{o p}}{\operatorname{hocolim}}|\tau \downarrow E|,
$$

indexed by the opposite category $\left(\mathcal{F}^{e}\right)^{\text {op }}$ since $E \mapsto|\tau \downarrow E|$ is the left homotopy Kan extension of the trivial functor over $\tau$. By definition, $\tau \downarrow E$ is the overcategory whose objects are the triples $\left(P, E^{\prime}, \alpha\right)$ for $\left(P, E^{\prime}\right)$ in $\widehat{\mathcal{L}}$ and $\alpha \in \operatorname{Hom}_{\mathcal{F} e}\left(E, E^{\prime}\right)$, and $\bar{C}_{\mathcal{L}}(E)$ can be identified with the full subcategory of those triples $\left(P, E^{\prime}, \alpha\right)$ for which $\alpha \in \operatorname{Iso}_{\mathcal{F}}\left(E, E^{\prime}\right)$. There is an obvious deformation retraction of $\tau \downarrow E$ to $\bar{C}_{\mathcal{L}}(E)$ which sends $\left(P, E^{\prime}, \alpha\right)$ to $(P, \alpha(E), \alpha)$, and this proves the first statement.

To prove the last statement, note that since $E$ is fully centralized in $\mathcal{F}$, any isomorphism $E^{\prime} \stackrel{\cong}{\longrightarrow} E$ in $\mathcal{F}$ extends to a homomorphism defined on $C_{S}\left(E^{\prime}\right)$. Hence each object in $\bar{C}_{\mathcal{L}}(E)$ is isomorphic to an object in the subcategory $C_{\mathcal{L}}(E)$, and so $\left|C_{\mathcal{L}}(E)\right|$ is a deformation retract of $\left|\bar{C}_{\mathcal{L}}(E)\right|$.

\section{Obstruction theory AND higher Limits}

We now consider the obstructions to the existence and uniqueness of centric linking systems associated to a given fusion system. These will be shown to lie in 
certain higher limits of the functor

$$
\mathcal{Z}=\mathcal{Z}_{\mathcal{F}}: \mathcal{O}^{c}(\mathcal{F})^{\text {op }} \longrightarrow A b
$$

defined for any fusion system $\mathcal{F}$ by setting $\mathcal{Z}_{\mathcal{F}}(P)=Z(P)$ and

$$
\mathcal{Z}_{\mathcal{F}}(P \stackrel{\varphi}{\longrightarrow} Q)=\left(Z(Q) \stackrel{\text { incl }}{\longrightarrow} Z(\varphi(P)) \stackrel{\varphi^{-1}}{\longrightarrow} Z(P)\right) .
$$

(Note that $Z(Q) \leq Z(\varphi(P))$ since $Q$ is $\mathcal{F}$-centric.) After proving this, we look more closely at techniques for computing in general higher limits of functors over such orbit categories. These will be important, not only for showing the existence of associated centric linking systems, but also later when describing certain spaces of maps to classifying spaces of $p$-local finite groups.

The obstructions defined by the following proposition are similar to those described by Hoff [Hf].

Proposition 3.1. Fix a saturated fusion system $\mathcal{F}$ over the p-group $S$. Then there is an element $\eta(\mathcal{F}) \in \lim ^{3}(\mathcal{Z})$ such that $\mathcal{F}$ has an associated centric linking system $\mathcal{O}^{c}(\mathcal{F})$

if and only if $\eta(\mathcal{F})=0$. Also, if there are any centric linking systems associated to $\mathcal{F}$, then the group $\lim ^{2}(\mathcal{Z})$ acts freely and transitively on the set of all isomorphism $\mathcal{O}^{c}(\mathcal{F})$

classes of centric linking systems associated to $\mathcal{F}$; i.e., on the set of all isomorphism classes of triples $(\mathcal{L}, \pi, \delta)$ as in Definition 1.7 .

Proof. The obstruction to the existence of an associated centric linking system will be handled in Step 1, and the action of $\lim ^{2}(\mathcal{Z})$ in Step 2. Let $C^{*}\left(\mathcal{O}^{c}(\mathcal{F}) ; \mathcal{Z}\right)$ denote the normalized chain complex for $\mathcal{Z}$ :

$$
C^{n}\left(\mathcal{O}^{c}(\mathcal{F}) ; \mathcal{Z}\right)=\prod_{P_{0} \rightarrow \cdots \rightarrow P_{n}} \mathcal{Z}\left(P_{0}\right)
$$

where the product is taken over all composable sequences of nonidentity morphisms. For simplicity, we regard cochains as functions defined on all sequences of morphisms, which send a sequence to $1 \in \mathcal{Z}\left(P_{0}\right)$ if any of the morphisms is an identity. Then

$$
\lim _{\mathcal{O}^{c}(\mathcal{F})}^{i}(\mathcal{Z}) \cong H^{i}\left(C^{*}\left(\mathcal{O}^{c}(\mathcal{F}) ; \mathcal{Z}\right), \delta\right)
$$

where $\delta$ is the obvious coboundary map, by the same argument as that given for the unnormalized chain complex in [GZ, Appendix II, Proposition 3.3] or [O], Lemma 2].

Step 1: Fix a section $\sigma: \operatorname{Mor}\left(\mathcal{O}^{c}(\mathcal{F})\right) \longrightarrow \operatorname{Mor}\left(\mathcal{F}^{c}\right)$ which sends identity maps to identity maps, and write $\widetilde{\varphi}=\sigma(\varphi)$ for short. For each pair of $\mathcal{F}$-centric subgroups $P, Q \leq S$, set

$$
X(P, Q)=Q \times \operatorname{Mor}_{\mathcal{O}^{c}(\mathcal{F})}(P, Q)
$$

and define

$$
X(P, Q) \stackrel{\pi_{\sigma}^{P, Q}}{\longrightarrow} \operatorname{Hom}_{\mathcal{F}}(P, Q)
$$

by setting

$$
\pi_{\sigma}^{P, Q}(g, \varphi)=c_{g} \circ \widetilde{\varphi} .
$$

For each composable pair of morphisms $P \stackrel{\varphi}{\longrightarrow} Q \stackrel{\psi}{\longrightarrow} R$ in the orbit category, choose some $t(\varphi, \psi) \in R$ such that

$$
\widetilde{\psi} \circ \widetilde{\varphi}=c_{t(\varphi, \psi)} \circ \widetilde{\psi \varphi}
$$


and such that

$$
t(\varphi, \psi)=1 \quad \text { if } \varphi=\operatorname{Id}_{Q} \text { or } \psi=\operatorname{Id}_{Q} .
$$

Define maps

$$
X(Q, R) \times X(P, Q) \stackrel{*}{\longrightarrow} X(P, R)
$$

by setting

$$
(h, \psi) *(g, \varphi)=(h \cdot \widetilde{\psi}(g) \cdot t(\varphi, \psi), \psi \varphi) .
$$

Definition of $u(\varphi, \psi, \chi)$ : By definition, if $P, Q \leq S$, and $\pi_{\sigma}^{P, Q}(g, \varphi)=\pi_{\sigma}^{P, Q}\left(g^{\prime}, \varphi\right)$ for some $g, g^{\prime} \in Q$ and $\varphi \in \operatorname{Rep}_{\mathcal{F}}(P, Q)$, then $g^{-1} g^{\prime} \in Z(\widetilde{\varphi}(P))$ since $P$ is $\mathcal{F}$-centric, and $(g, \varphi)=\left(g^{\prime}, \varphi\right) *\left(u, \operatorname{Id}_{P}\right)$ for some $u \in Z(P)$. Also, by construction, the following square commutes for each triple of objects $P, Q, R$ :

$$
\begin{aligned}
& X(Q, R) \times X(P, Q) \longrightarrow * \\
& \pi_{\sigma}^{Q, R} \times \pi_{\sigma}^{P, Q} \mid X(P, R) \\
& \operatorname{Hom}_{\mathcal{F}}(Q, R) \times \operatorname{Hom}_{\mathcal{F}}(P, Q) \stackrel{\text { composition }}{\longrightarrow} \pi_{\sigma}^{P, R} \downarrow \\
& \operatorname{Hom}_{\mathcal{F}}(P, R) .
\end{aligned}
$$

Hence for each triple of composable maps

$$
P \stackrel{\varphi}{\longrightarrow} P^{\prime} \stackrel{\psi}{\longrightarrow} Q \stackrel{\chi}{\longrightarrow} R
$$

in the orbit category, there is a unique element $u(\varphi, \psi, \chi)=u_{\sigma, t}(\varphi, \psi, \chi) \in Z(P)$ such that

$$
((1, \chi) *(1, \psi)) *(1, \varphi)=[(1, \chi) *((1, \psi) *(1, \varphi))] *\left(u(\varphi, \psi, \chi), \operatorname{Id}_{P}\right) .
$$

We regard $u \in C^{3}\left(\mathcal{O}^{c}(\mathcal{F}) ; \mathcal{Z}\right)$ as a normalized 3-cochain. Upon substituting formula (3) into (4), we get the following formula for $u(\varphi, \psi, \chi)$ :

$$
\widetilde{\chi \psi \varphi}(u(\varphi, \psi, \chi))=t(\psi \varphi, \chi)^{-1} \cdot \widetilde{\chi}(t(\varphi, \psi))^{-1} \cdot t(\psi, \chi) \cdot t(\varphi, \chi \psi) .
$$

After combining this with (3) again, we get that for each $g \in P^{\prime}, h \in Q$, and $k \in R$,

$$
((k, \chi) *(h, \psi)) *(g, \varphi)=[(k, \chi) *((h, \psi) *(g, \varphi))] *\left(u(\varphi, \psi, \chi), \operatorname{Id}_{P}\right) .
$$

Proof that $u$ is a 3-cocycle: Fix a sequence of morphisms

$$
P \stackrel{\varphi}{\longrightarrow} P^{\prime} \stackrel{\psi}{\longrightarrow} Q \stackrel{\chi}{\longrightarrow} Q^{\prime} \stackrel{\omega}{\longrightarrow} R
$$

in $\mathcal{O}^{c}(\mathcal{F})$. Then

$$
\begin{aligned}
& \delta u(\varphi, \psi, \chi, \omega) \\
& \quad=u(\varphi, \psi, \omega \chi)^{-1} \cdot u(\psi \varphi, \chi, \omega)^{-1} \cdot u(\varphi, \psi, \chi) \cdot u(\varphi, \chi \psi, \omega) \cdot \widetilde{\varphi}^{-1}(u(\psi, \chi, \omega))
\end{aligned}
$$

(each term lies in the abelian group $Z(P)$ ), and we must show that this vanishes.

Set $\Phi=\sigma(\omega \chi \psi \varphi) \in \operatorname{Hom}(P, R)$ for short. Then by (1),

$$
\begin{aligned}
\Phi(u(\varphi, \psi, \chi)) & =t(\chi \psi \varphi, \omega)^{-1} \cdot(\widetilde{\omega} \circ \widetilde{\chi \psi \varphi}(u(\varphi, \psi, \chi))) \cdot t(\chi \psi \varphi, \omega), \\
\Phi \circ \widetilde{\varphi}^{-1}(u(\psi, \chi, \omega)) & =t(\varphi, \omega \chi \psi)^{-1} \cdot(\widetilde{\omega \chi \psi}(u(\psi, \chi, \omega))) \cdot t(\varphi, \omega \chi \psi) .
\end{aligned}
$$


Together with (5), this gives the formulas

$$
\begin{aligned}
\Phi(u(\varphi, \psi, \chi))= & t(\chi \psi \varphi, \omega)^{-1} \cdot \widetilde{\omega}(t(\psi \varphi, \chi))^{-1} \\
& \cdot\left(t(\chi, \omega) \cdot \widetilde{\omega}(t(\varphi, \psi))^{-1} \cdot t(\chi, \omega)^{-1}\right) \\
\cdot \widetilde{\omega}(t(\psi, \chi)) \cdot \widetilde{\omega}(t(\varphi, \chi \psi)) \cdot t(\chi \psi \varphi, \omega), & \\
\Phi(u(\varphi, \psi, \omega \chi))= & t(\psi \varphi, \omega \chi)^{-1} \cdot \widetilde{\omega \chi}(t(\varphi, \psi))^{-1} \cdot t(\psi, \omega \chi) \cdot t(\varphi, \omega \chi \psi), \\
\Phi(u(\varphi, \chi \psi, \omega))= & t(\chi \psi \varphi, \omega)^{-1} \cdot \widetilde{\omega}(t(\varphi, \chi \psi))^{-1} \cdot t(\chi \psi, \omega) \cdot t(\varphi, \omega \chi \psi), \\
\Phi(u(\psi \varphi, \chi, \omega))= & t(\chi \psi \varphi, \omega)^{-1} \cdot \widetilde{\omega}(t(\psi \varphi, \chi))^{-1} \cdot t(\chi, \omega) \cdot t(\psi \varphi, \omega \chi), \\
\Phi\left(\widetilde{\varphi}^{-1}(u(\psi, \chi, \omega))\right)= & t(\varphi, \omega \chi \psi)^{-1} \cdot t(\chi \psi, \omega)^{-1} \\
& \cdot \widetilde{\omega}(t(\psi, \chi))^{-1} \cdot t(\chi, \omega) \cdot t(\psi, \omega \chi) \cdot t(\varphi, \omega \chi \psi) .
\end{aligned}
$$

Upon substituting these into (7), we get that

$$
\Phi(\delta u(\varphi, \psi, \chi, \omega))=1,
$$

and hence that $\delta u(\varphi, \psi, \chi, \omega)=1$.

To see this more geometrically, consider the following cube, where each vertex is labelled by a homomorphism $P \longrightarrow R$ in the conjugacy class $\omega \chi \psi \varphi \in \operatorname{Rep}(P, R)$, and where each edge is labelled with an element of $R$ :

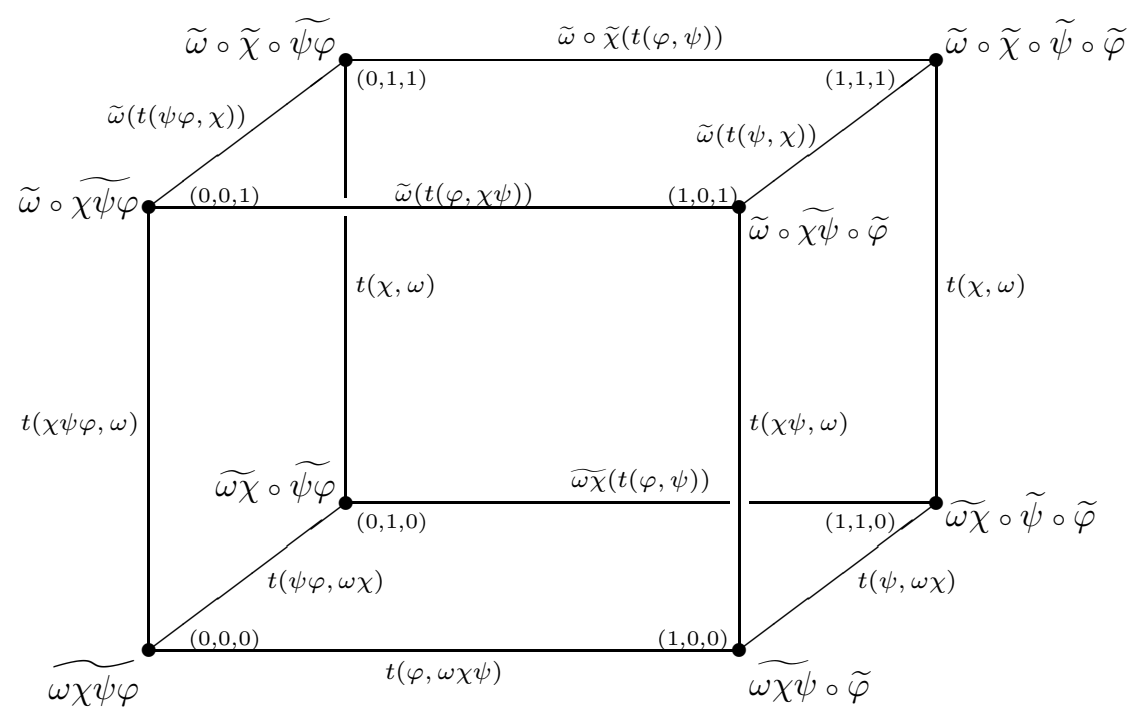

The vertices of the cube are given the coordinatewise partial ordering, and we regard each edge as being oriented from the smaller to the larger vertex. Whenever an edge in the cube is labelled by $g \in R$ and its endpoints by $f_{0}, f_{1} \in \operatorname{Hom}(P, R)$ (in that order), then $c_{g} \circ f_{0}=f_{1}$. In particular, the product of the successive edges of any loop in the diagram (multiplied from right to left, and where an element is inverted if the orientation is reversed) lies in $f(Z(P))$ if $f \in \operatorname{Hom}(P, R)$ is the label of the basepoint of the loop.

The "back face" $(*, 1, *)$ represents an identity in $R$ (by (1)). Each of the other five faces, when regarded as a loop based at $(0,0,0)$, represents one of the terms in $\Phi(\delta u(\varphi, \psi, \chi, \omega))$. For example, the two faces $(*, *, 1)$ and $(1, *, *)$ represent the first and last formulas in (8), with extra terms coming from the edge which connects these faces to the vertex $(0,0,0)$. The other three formulas in (8) correspond to 
the three faces which contain $(0,0,0)$. Using this picture, we see directly that the product in (7) (rather, its image under $\Phi$ ) vanishes, and hence that $\delta u=1$.

Independence of the choice of $t(\varphi, \psi)$ : Let $t^{\prime}(\varphi, \psi)$, for each composable pair of morphisms in $\mathcal{O}^{c}(\mathcal{F})$, be another collection of elements which satisfy (1). Let $u^{\prime}=u_{\sigma, t^{\prime}} \in Z^{3}\left(\mathcal{O}^{c}(\mathcal{F}) ; \mathcal{Z}\right)$ be the 3-cochain defined using (3) and (4) (after replacing $t$ by $t^{\prime}$ ). By the previous argument, $u^{\prime}$ is a 3 -cocycle, and (5) now takes the form

$$
t^{\prime}(\psi, \chi) \cdot t^{\prime}(\varphi, \chi \psi)=\widetilde{\chi}\left(t^{\prime}(\varphi, \psi)\right) \cdot t^{\prime}(\psi \varphi, \chi) \cdot \widetilde{\chi \psi \varphi}\left(u^{\prime}(\varphi, \psi, \chi)\right) .
$$

For each composable sequence $P \stackrel{\varphi}{\longrightarrow} Q \stackrel{\psi}{\longrightarrow} R$, conjugation by $t^{\prime}(\varphi, \psi)$ and by $t(\varphi, \psi)$ define the same automorphism of $R$, and hence there is a unique element $c(\varphi, \psi)$ such that

$$
t^{\prime}(\varphi, \psi)=t(\varphi, \psi) \cdot \widetilde{\psi \varphi}(c(\varphi, \psi)) .
$$

Then $c \in C^{2}\left(\mathcal{O}^{c}(\mathcal{F}) ; \mathcal{Z}\right)$ is a (normalized) 2-cochain. Upon substituting (9) into $(8)$, and using the relations

$$
\widetilde{\chi \psi}=c_{t(\varphi, \chi \psi)} \circ \widetilde{\chi \psi \varphi} \circ \widetilde{\varphi}^{-1} \quad \text { and } \quad \tilde{\chi} \circ \widetilde{\psi \varphi}=c_{t(\chi, \psi \varphi)} \circ \widetilde{\chi \psi \varphi},
$$

we get the relation

$$
\begin{aligned}
& t(\psi, \chi) \cdot t(\varphi, \chi \psi) \cdot \widetilde{\chi \psi \varphi}\left(\widetilde{\varphi}^{-1} c(\psi, \chi)\right) \cdot \widetilde{\chi \psi \varphi}(c(\varphi, \chi \psi)) \\
& \quad=\tilde{\chi}(t(\varphi, \psi)) \cdot t(\psi \varphi, \chi) \cdot \widetilde{\chi \psi \varphi}(c(\varphi, \psi)) \cdot \widetilde{\chi \psi \varphi}(c(\psi \varphi, \chi)) \cdot \widetilde{\chi \psi \varphi}\left(u^{\prime}(\varphi, \psi, \chi)\right) .
\end{aligned}
$$

The four factors in this equation which are not in the image of $\widetilde{\chi \psi \varphi}$ can be replaced by $\widetilde{\chi \psi \varphi}(u(\varphi, \psi, \chi))$ using (5), and we thus get that

$$
u(\varphi, \psi, \chi) \cdot \widetilde{\varphi}^{-1}(c(\psi, \chi)) \cdot c(\varphi, \chi \psi)=c(\varphi, \psi) \cdot c(\psi \varphi, \chi) \cdot u^{\prime}(\varphi, \psi, \chi) .
$$

Since all terms in this equation lie in $Z(P)$, this shows that

$$
u^{-1} \cdot u^{\prime}=\delta c .
$$

A different choice of $t$ thus results in changing $u$ by a coboundary, and does not change the class $[u] \in \lim ^{3}(\mathcal{Z})$.

Independence of the choice of $\sigma$ : This follows upon observing that under a different choice of section $\sigma^{\prime}$, the resulting sets and maps

$$
X^{\prime}(P, Q) \stackrel{\pi_{\sigma^{\prime}}^{P, Q}}{\longrightarrow} \operatorname{Hom}_{\mathcal{F}}(P, Q)
$$

can be identified with $X(P, Q)$ and $\pi_{\sigma}^{P, Q}$ in an obvious way. This induces elements $t^{\prime}(\varphi, \psi)$ such that the $X^{\prime}$ and the $X$ have the same composition under these identifications. But by (6), this shows that $u_{\sigma^{\prime}, t^{\prime}}=u_{\sigma, t}$, and thus that $[u]$ is not changed by this different choice of section.

Existence of a centric linking system if $[u]=0$ : Formula (11) also shows that if $u=u_{\sigma, t}$ is a coboundary, then we can choose $t^{\prime}$ such that $u_{\sigma, t^{\prime}}=0$, and hence get a category $\mathcal{L}$ with $\operatorname{Mor}_{\mathcal{L}}(P, Q)=X(P, Q)$ and with composition defined using (3) but using $t^{\prime}(\varphi, \psi)$ instead of $t(\varphi, \psi)$. In this case, we set $\widehat{g}=(g$, Id $)$ for $g \in P$, and $\pi(g, \varphi)=c_{g} \circ \widetilde{\varphi}$ for $\varphi \in \operatorname{Rep}_{\mathcal{F}}(P, Q)$ and $g \in Q$. Conditions (A-C) are easily checked. For example, for any $(a, \varphi) \in X(P, Q)$ and any $g \in P$,

$$
(x, \varphi) *\left(g, \operatorname{Id}_{P}\right)=(x \cdot \widetilde{\varphi}(g), \varphi)=\left(x \widetilde{\varphi}(g) x^{-1}, \operatorname{Id}_{Q}\right) *(x, \varphi)=\left(c_{x} \circ \widetilde{\varphi}(g), \operatorname{Id}_{Q}\right) *(x, \varphi)
$$


by $(2)$ and (3), and this implies $(\mathrm{C})$. So $\mathcal{L}$ is a centric linking system associated to $\mathcal{F}$.

Vanishing of $[u]$ if there is a centric linking system: Let $\mathcal{L}$ be any centric linking system associated to $\mathcal{F}$, and fix a section $\sigma$ as above. This can be lifted to a section $\operatorname{Mor}\left(\mathcal{O}^{c}(\mathcal{F})\right) \longrightarrow \operatorname{Mor}(\mathcal{L})$, which in turn defines bijections $X(P, Q) \cong \operatorname{Mor}_{\mathcal{L}}(P, Q)$ in the obvious way. Since $(\mathrm{C})$ holds, composition in $\mathcal{L}$ must correspond to multiplication of the $X(P, Q)$ (as defined by (3)) for some choice of elements $t(\varphi, \psi)$; and thus $u_{\sigma, t}=0$ in this case. This shows that $[u]=0$ whenever there exist associated centric linking systems.

Step 2: Assume that $\mathcal{L}_{1} \stackrel{\pi_{1}}{\longrightarrow} \mathcal{F}^{c}$ and $\mathcal{L}_{2} \stackrel{\pi_{2}}{\longrightarrow} \mathcal{F}^{c}$ are two centric linking systems associated to $\mathcal{F}$. Let $\operatorname{Mor}\left(\mathcal{O}^{c}(\mathcal{F})\right) \stackrel{\sigma}{\longrightarrow} \operatorname{Mor}\left(\mathcal{F}^{c}\right)$ be as above, and fix sections

$$
\operatorname{Mor}\left(\mathcal{O}^{c}(\mathcal{F})\right) \stackrel{\widetilde{\sigma}_{1}}{\longrightarrow} \operatorname{Mor}\left(\mathcal{L}_{1}\right) \quad \text { and } \quad \operatorname{Mor}\left(\mathcal{O}^{c}(\mathcal{F})\right) \stackrel{\widetilde{\sigma}_{2}}{\longrightarrow} \operatorname{Mor}\left(\mathcal{L}_{2}\right)
$$

which send identity morphisms to identity morphisms and such that $\pi_{i} \circ \widetilde{\sigma}_{i}=\sigma$ for $i=1,2$. For each $P \stackrel{\varphi}{\longrightarrow} Q \stackrel{\psi}{\longrightarrow} R$ in $\mathcal{O}^{c}(\mathcal{F})$, let $t_{i}(\varphi, \psi) \in R$ be the element (unique by Lemma1.10(b)) such that

$$
\widetilde{\sigma}_{i}(\psi) \circ \widetilde{\sigma}_{i}(\varphi)=\widehat{t_{i}(\varphi, \psi)} \circ \widetilde{\sigma}_{i}(\psi \varphi)
$$

in $\mathcal{L}_{i}(i=1,2)$. These satisfy (1) and (2) above, as well as (3) when we identify $(g, \varphi)=\widehat{g} \circ \widetilde{\sigma}_{i}(\varphi)$. There is thus an element $c(\varphi, \psi) \in Z(P)$ such that

$$
t_{2}(\varphi, \psi)=t_{1}(\varphi, \psi) \cdot \widetilde{\psi \varphi}(c(\varphi, \psi)) .
$$

By (11) $\left(u_{1}=u_{2}=1\right.$ in this case since $\mathcal{L}_{1}$ and $\mathcal{L}_{2}$ are actual categories), $c$ is a (normalized) 2-cocycle.

Now assume that $\widetilde{\sigma}_{i}^{\prime}(i=1$ or 2 ) is another section (over the fixed section $\sigma$ ), and define elements $t_{i}^{\prime}(\varphi, \psi)$ using (12). By condition (A), there is a unique 1-cochain $w \in C^{1}\left(\mathcal{O}^{c}(\mathcal{F}) ; \mathcal{Z}\right)$ such that for each morphism $\varphi$ in $\mathcal{O}^{c}(\mathcal{F}), \widetilde{\sigma}_{i}^{\prime}(\varphi)=\widetilde{\sigma}_{i}^{\prime}(\varphi) \cdot w(\varphi)$. Upon substituting this into the definition of $t_{i}^{\prime}$, we get that

$$
\widetilde{\sigma}_{i}(\psi) \circ \widehat{w(\psi)} \circ \widetilde{\sigma}_{i}(\varphi) \circ \widehat{w(\varphi)}=\widehat{t_{i}^{\prime}(\varphi, \psi)} \circ \widetilde{\sigma}_{i}(\psi \varphi) \circ \widehat{w(\psi \varphi)} \in \operatorname{Mor}_{\mathcal{L}_{i}}(P, R)
$$

for each $P \stackrel{\varphi}{\longrightarrow} Q \stackrel{\psi}{\longrightarrow} R$ in $\mathcal{O}^{c}(\mathcal{F})$; and hence (using condition $(\mathrm{C})$ ) that

$$
\delta_{R}(\widetilde{\psi}(w(\psi)) \cdot \widetilde{\psi} \widetilde{\varphi}(w(\varphi))) \circ \widetilde{\sigma}_{i}(\psi) \circ \widetilde{\sigma}_{i}(\varphi)=\delta_{R}\left(t_{i}^{\prime}(\varphi, \psi) \cdot \widetilde{\psi \varphi}(w(\psi \varphi))\right) \circ \widetilde{\sigma}_{i}(\psi \varphi) .
$$

After substituting (12) into this we get

$$
\widetilde{\psi}(w(\psi)) \cdot \widetilde{\psi} \widetilde{\varphi}(w(\varphi)) \cdot t_{i}(\varphi, \psi)=t_{i}^{\prime}(\varphi, \psi) \cdot \widetilde{\psi \varphi}(w(\psi \varphi)) \in R .
$$

From this, together with the relation $c_{t_{i}(\varphi, \psi)} \circ \widetilde{\psi \varphi}=\widetilde{\psi} \circ \widetilde{\varphi}$, it follows that

$$
\begin{aligned}
t_{i}(\varphi, \psi)^{-1} \cdot t_{i}^{\prime}(\varphi, \psi) & =c_{t_{i}(\varphi, \psi)}^{-1}(\widetilde{\psi}(w(\psi)) \cdot \widetilde{\psi} \widetilde{\varphi}(w(\varphi))) \cdot \widetilde{\psi \varphi}\left(w(\psi \varphi)^{-1}\right) \\
& =\widetilde{\psi \varphi}\left(\widetilde{\varphi}^{-1}(w(\psi)) \cdot w(\varphi) \cdot w(\psi \varphi)^{-1}\right)=\widetilde{\psi \varphi}(\delta w(\varphi, \psi)) .
\end{aligned}
$$

In other words, a change in $\widetilde{\sigma}_{i}$ corresponds to changing $c$ by a coboundary, and hence the class $[c] \in \lim ^{2}(\mathcal{Z})$ is uniquely defined (depending only on $\mathcal{L}_{1}$ and $\mathcal{L}_{2}$ ). Also, $\mathcal{L}_{1}$ and $\mathcal{L}_{2}$ are isomorphic as categories over $\mathcal{O}^{c}(\mathcal{F})$ (i.e., there is a functor $\mathcal{L}_{1} \longrightarrow \mathcal{L}_{2}$ which is bijective on objects and morphisms and commutes with the $\pi_{i}$ and the $\left.P \longrightarrow \operatorname{Aut}_{\mathcal{L}_{i}}(P)\right)$ if and only if $t_{1}=t_{2}$ for some choice of these sections, if and only if $[c]=0$. Finally, for fixed $\mathcal{L}_{1}$ and $\widetilde{\sigma}_{1}$, any 2-cocycle $c$ can be realized by some appropriate choice of $\mathcal{L}_{2}$ and $\widetilde{\sigma}_{2}$ : first define $t_{2}$ using (13), and then define 
$\mathcal{L}_{2}$ using (3). This finishes the proof that $\lim ^{2}(\mathcal{Z})$ acts freely and transitively on the set of isomorphism classes of centric linking systems associated to $\mathcal{F}$.

We now look more closely at higher limits of functors over an $\mathcal{F}$-centric orbit category $\mathcal{O}^{c}(\mathcal{F})$ of a saturated fusion system $\mathcal{F}$, and show that they can be computed using the same techniques as those already used to compute higher limits over orbit categories of finite groups. The main tools for doing this are certain graded groups $\Lambda^{*}(\Gamma ; M)$, defined for any finite group $\Gamma$ and any $\mathbb{Z}_{(p)}[\Gamma]$-module $M$ by setting

$$
\Lambda^{*}(\Gamma ; M)={\underset{\mathcal{O}}{\lim _{p}(\Gamma)}}^{*}\left(F_{M}\right),
$$

where $F_{M}: \mathcal{O}_{p}(\Gamma) \longrightarrow \mathbb{Z}_{(p)}$-mod is the functor defined by setting $F_{M}(\Gamma / 1)=M$, and $F_{M}(\Gamma / P)=0$ for $p$-subgroups $1 \neq P \leq \Gamma$. Here, $F_{M}(\Gamma / 1)$ has the given action of $\operatorname{Aut}_{\mathcal{O}_{p}(G)}(\Gamma / 1)=\Gamma$.

Proposition 3.2. Let $\mathcal{F}$ be a saturated fusion system over $S$. Let

$$
\Phi: \mathcal{O}^{c}(\mathcal{F})^{\text {op }} \longrightarrow \mathbb{Z}_{(p)} \text {-mod }
$$

be any functor which vanishes except on the isomorphism class of some fixed $\mathcal{F}$ centric subgroup $Q \leq S$. Then

$$
\underset{\mathcal{O}^{c}(\mathcal{F})}{\lim ^{*}}(\Phi) \cong \Lambda^{*}\left(\operatorname{Out}_{\mathcal{F}}(Q) ; \Phi(Q)\right)
$$

Proof. Since the result is independent of the choice of $Q$ in its $\mathcal{F}$-conjugacy class, we can assume that $Q$ is fully normalized in $\mathcal{F}$. In particular,

$$
\operatorname{Out}_{S}(Q) \in \operatorname{Syl}_{p}\left(\operatorname{Out}_{\mathcal{F}}(Q)\right) \text {. }
$$

Set $\Gamma=\operatorname{Out}_{\mathcal{F}}(Q)$ and $\Gamma_{p}=\operatorname{Out}_{S}(Q)$ for short.

We want to compare higher limits of functors over the two orbit categories $\mathcal{O}_{p}(\Gamma)$ and $\mathcal{O}^{c}(\mathcal{F})$, by constructing adjoint functors between them. However, before doing this, it is first necessary to modify and extend these categories. Let $\mathcal{O}_{\Gamma_{p}}(\Gamma)$ be the full subcategory $\mathcal{O}_{p}(\Gamma)$ consisting of all orbits $\Gamma / \Gamma^{\prime}$ with $\Gamma^{\prime} \leq \Gamma_{p}$. This is clearly equivalent to $\mathcal{O}_{p}(\Gamma)$ itself. Let $\mathcal{O}^{c}(\mathcal{F})_{\amalg}$ and $\mathcal{O}_{\Gamma_{p}}(\Gamma)_{\amalg}$ be the categories of formal finite "sums" of objects in $\mathcal{O}^{c}(\mathcal{F})$ and $\mathcal{O}_{\Gamma_{p}}(\Gamma)$, respectively, where a morphism sends each summand in the source object to exactly one summand in the target. Finally, let $\operatorname{Set}_{p}(\Gamma) \cong \mathcal{O}_{p}(\Gamma)_{\amalg}$ be the category whose objects are finite left $\Gamma$-sets whose isotropy subgroups are $p$-groups. Morphisms in $\mathfrak{S e t}_{p}(\Gamma)$ are $\Gamma$-maps. The inclusion $i: \mathcal{O}_{\Gamma_{p}}(\Gamma)_{\amalg} \longrightarrow \mathfrak{S e t}_{p}(\Gamma)$ is an equivalence of categories, and so we can choose an inverse

$$
\mathcal{O}_{\Gamma_{p}}(\Gamma)_{\amalg} \stackrel{i}{\longleftarrow} \operatorname{Set}_{p}(\Gamma)
$$

(inverse up to natural isomorphism) by assigning a fixed orbit $s(X)$ of $\mathcal{O}_{\Gamma_{p}}(\Gamma)$ to each isomorphism class of orbits $X$ of $\mathfrak{S e t}_{p}(\Gamma)$. (More precisely, we do this after replacing $\mathfrak{S e t}_{p}(\Gamma)$ by an equivalent small category which contains $\mathcal{O}_{\Gamma_{p}}(\Gamma)_{\amalg}$, and also contains the sets $\operatorname{Rep}_{\mathcal{F}}(P, Q)$ for all $P \leq S$ with the left $\Gamma$-action given by composition as described below.)

Recall that morphisms in $\mathcal{O}^{c}(\mathcal{F})$ are given by the formula

$$
\operatorname{Mor}_{\mathcal{O}^{c}(\mathcal{F})}(P, Q)=\operatorname{Rep}_{\mathcal{F}}(P, Q) \stackrel{\text { def }}{=} Q \backslash \operatorname{Hom}_{\mathcal{F}}(P, Q) .
$$


Define functors $\bar{\alpha}$ and $\beta$,

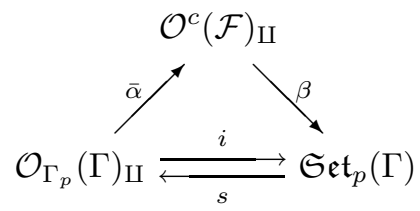

as follows. For each $\Gamma^{\prime} \leq \Gamma_{p}$, set

$$
\bar{\alpha}\left(\Gamma / \Gamma^{\prime}\right)=N_{S}^{\Gamma^{\prime}}(Q) \stackrel{\text { def }}{=}\left\{x \in N_{S}(Q) \mid\left[g \mapsto x g x^{-1}\right] \in \Gamma^{\prime}\right\}
$$

(any subgroup of $S$ which contains an $\mathcal{F}$-centric subgroup is also $\mathcal{F}$-centric). Set $\beta(P)=\operatorname{Rep}_{\mathcal{F}}(Q, P)$ for each $P \leq S$, with the left action of $\Gamma=\operatorname{Out}_{\mathcal{F}}(Q)$ induced by right composition. More precisely, $\gamma \in \Gamma$ acts on $\operatorname{Rep}_{\mathcal{F}}(Q, P)$ via right composition with $\gamma^{-1}$, and this action extends to morphisms in the obvious way. Note that the isotropy subgroup of any $\varphi \in \operatorname{Rep}_{\mathcal{F}}(Q, P)$ is a $p$-group. We will construct an isomorphism

$$
\operatorname{Mor}_{\mathcal{O}^{c}(\mathcal{F})}\left(\bar{\alpha}\left(\Gamma / \Gamma^{\prime}\right), P\right) \stackrel{\cong}{\longrightarrow} \operatorname{Mor}_{\mathfrak{S e t}_{p}(\Gamma)}\left(i\left(\Gamma / \Gamma^{\prime}\right), \beta(P)\right)
$$

which is natural for all $\Gamma / \Gamma^{\prime}$ in $\mathcal{O}_{\Gamma_{p}}(\Gamma)$ and all $P$ in $\mathcal{O}^{c}(\mathcal{F})$. When combined with the natural isomorphism

$$
\operatorname{Mor}_{\mathfrak{S e t}_{p}(\Gamma)}(i(s(X)), \beta(P)) \cong \operatorname{Mor}_{\mathfrak{S e t}_{p}(\Gamma)}(X, \beta(P))
$$

for each $X$ in $\mathcal{O}^{c}(\mathcal{F})_{\amalg}$, and upon defining $\alpha=\bar{\alpha} \circ s$, this shows that the functors

$$
\mathfrak{S e t}_{p}(\Gamma) \underset{\beta}{\stackrel{\alpha}{\rightleftarrows}} \mathcal{O}^{c}(\mathcal{F})_{\amalg}
$$

are adjoint.

We now construct the isomorphism in (1). Fix subgroups $\Gamma^{\prime} \leq \Gamma_{p}$ and $P \leq S$ such that $P$ is $\mathcal{F}$-centric. Since $Q \leq N_{S}^{\Gamma^{\prime}}(Q)$, there is a map

$$
\mu: \operatorname{Rep}_{\mathcal{F}}\left(N_{S}^{\Gamma^{\prime}}(Q), P\right) \longrightarrow \operatorname{Rep}_{\mathcal{F}}(Q, P)
$$

defined by restriction, which is injective by Proposition $A .8$ (and since $Q$ is $\mathcal{F}$ centric). Also, $\Gamma=\operatorname{Out}_{\mathcal{F}}(Q)$ acts on $\operatorname{Rep}_{\mathcal{F}}(Q, P)$ by composition, and $\operatorname{Im}(\mu) \leq$ $\operatorname{Rep}_{\mathcal{F}}(Q, P)^{\Gamma^{\prime}}$ since $\Gamma^{\prime}$ is the stabilizer of the inclusion $Q \hookrightarrow N_{S}^{\Gamma^{\prime}}(Q)$. Moreover, any element in $\operatorname{Rep}_{\mathcal{F}}(Q, P)$ fixed by $\Gamma^{\prime}$ extends to an element of $\operatorname{Rep}_{\mathcal{F}}\left(N_{S}^{\Gamma^{\prime}}(Q), P\right)$ by condition (II) in Definition 1.2 (In fact, condition (II) only shows that any $\Gamma^{\prime}$-invariant morphism in $\operatorname{Rep}_{\mathcal{F}}(Q, P)$ extends to an element of $\operatorname{Rep}_{\mathcal{F}}\left(N_{S}^{\Gamma^{\prime}}(Q), S\right)$, but using the fact that $Q$ is $\mathcal{F}$-centric one shows that its image is contained in $P$.) This shows that $\mu$ restricts to a bijection

$$
\operatorname{Rep}_{\mathcal{F}}\left(N_{S}^{\Gamma^{\prime}}(Q), P\right) \underset{\cong}{\stackrel{\mu_{0}}{\cong}} \operatorname{Rep}_{\mathcal{F}}(Q, P)^{\Gamma^{\prime}} \cong \operatorname{Map}_{\Gamma}\left(\Gamma / \Gamma^{\prime}, \operatorname{Rep}_{\mathcal{F}}(Q, P)\right) .
$$

This is natural in the first variable with respect to morphisms in

$$
\operatorname{Mor}_{\mathcal{O}_{\Gamma_{p}}(\Gamma)}\left(\Gamma / \Gamma^{\prime}, \Gamma / \Gamma^{\prime \prime}\right)
$$

and in the second variable with respect to morphisms in $\operatorname{Rep}_{\mathcal{F}}\left(P, P^{\prime}\right)$. This finishes the proof of (1), and hence the proof that $\alpha$ and $\beta$ are adjoint. 
For $\mathcal{C}=\mathcal{O}_{p}(\Gamma)$ or $\mathcal{O}^{c}(\mathcal{F})$, we let $\mathcal{C}$-mod be the category of functors $\mathcal{C}^{\text {op }} \longrightarrow A$ b. Since this is equivalent to the category of functors $\left(\mathcal{C}_{\amalg}\right)^{\text {op }} \longrightarrow A b$ which send disjoint unions to direct sums, composition with $\alpha$ and $\beta$ induces functors

$$
\mathcal{O}_{p}(\Gamma)-\bmod \underset{\beta^{*}}{\stackrel{\alpha^{*}}{\leftrightarrows}} \mathcal{O}^{c}(\mathcal{F})-\bmod
$$

Then $\alpha^{*}$ is a left adjoint to $\beta^{*}$, since $\alpha$ is a left adjoint to $\beta$. Also, $\alpha^{*}$ and $\beta^{*}$ both preserve exact sequences, and hence $\beta^{*}$ sends injectives to injectives.

Now let $\underline{\mathbb{Z}}$ denote the constant functor on $\mathcal{O}^{c}(\mathcal{F})^{\text {op }}$ which sends each object to $\mathbb{Z}$ and each morphism to the identity. Then $\alpha^{*} \underline{\mathbb{Z}}$ is the constant functor on $\mathcal{O}_{p}(\Gamma)$, since $\alpha$ sends objects of $\mathcal{O}_{p}(\Gamma)$ to objects of $\mathcal{O}^{c}(\mathcal{F})$ (not to formal sums of objects). If $D: \mathcal{O}^{c}(\mathcal{F})^{\text {op }} \longrightarrow A b$ is any functor, then

$$
{\underset{\mathcal{O}^{c}(\mathcal{F})}{ }}^{0}(D) \cong \operatorname{Hom}_{\mathcal{O}^{c}(\mathcal{F})-\bmod }(\underline{\mathbb{Z}}, D)
$$

and similarly for functors on $\mathcal{O}_{p}(\Gamma)$.

Let $\bar{\Phi}: \mathcal{O}_{p}(\Gamma)^{\text {op }} \longrightarrow \mathbb{Z}_{(p)}$-mod be the functor which sends the free orbit $\Gamma / 1$ to $\Phi(Q)$ (with the given action of $\Gamma$ ) and all other orbits to 0 . Then $\beta^{*}$ sends an injective resolution $I_{*}$ of $\bar{\Phi}$ to an injective resolution $\beta^{*} I_{*}$ of $\beta^{*}(\bar{\Phi})$. It follows that

$$
\begin{aligned}
\Lambda^{*}(\Gamma ; \Phi(Q)) \stackrel{\text { def }}{=}{\overleftarrow{\mathcal{O}_{p}(\Gamma)}}_{\lim ^{*}}(\bar{\Phi}) & \cong H^{*}\left(\operatorname{Mor}_{\mathcal{O}_{p}(\Gamma)-\bmod }\left(\alpha^{*} \underline{\mathbb{Z}}, I_{*}\right)\right) \\
& \cong H^{*}\left(\operatorname{Mor}_{\mathcal{O}^{c}(\mathcal{F})-\bmod }\left(\underline{\mathbb{Z}}, \beta^{*} I_{*}\right)\right) \cong{\overleftarrow{\mathcal{O}^{c}(\mathcal{F})}}_{\lim ^{*}\left(\beta^{*} \bar{\Phi}\right) .}
\end{aligned}
$$

By definition, $\bar{\Phi}=\alpha^{*} \Phi$, and it remains only to show that $\beta^{*}\left(\alpha^{*} \Phi\right) \cong \Phi$. For each $P \leq S$, choose a $\Gamma$-orbit decomposition

$$
\operatorname{Rep}_{\mathcal{F}}(Q, P)=\coprod_{i=1}^{m} \Gamma \cdot \varphi_{i}
$$

and let $\Gamma_{\varphi_{i}}$ denote the isotropy group of the $\Gamma$-action on $\varphi_{i}$. Then $s\left(\Gamma \cdot \varphi_{i}\right)=\Gamma / \Gamma_{i}^{\prime}$ for some $\Gamma_{i}^{\prime} \leq \Gamma_{p}$ which is $\Gamma$-conjugate to $\Gamma_{\varphi_{i}}$. Thus

$$
\alpha(\beta(P))=\bar{\alpha}\left(s\left(\operatorname{Rep}_{\mathcal{F}}(Q, P)\right)\right)=\bar{\alpha}\left(\coprod_{i=1}^{m} \Gamma / \Gamma_{i}^{\prime}\right)=\coprod_{i=1}^{m} N_{S}^{\Gamma_{i}^{\prime}}(Q),
$$

and so

$$
\beta^{*}\left(\alpha^{*} \Phi\right)(P) \cong \bigoplus_{i=1}^{m} \Phi\left(N_{S}^{\Gamma_{i}^{\prime}}(Q)\right)
$$

If $Q$ is not isomorphic to $P$, then for each $\varphi \in \operatorname{Rep}_{\mathcal{F}}(Q, P), 1 \neq \varphi^{-1}\left(\operatorname{Out}_{P}(\varphi Q)\right) \varphi \leq$ $\Gamma_{\varphi}$. So the action of $\Gamma$ is not free on any orbit of $\operatorname{Rep}_{\mathcal{F}}(Q, P)$, hence $N_{S}^{\Gamma^{\prime}}(Q) \supsetneqq Q$ for each $i, \Phi\left(N_{S}^{\Gamma_{i}^{\prime}}(Q)\right)=0$ by the assumption on $\Phi$, and thus $\beta^{*}\left(\alpha^{*} \Phi\right)(P)=0$. Finally, if $Q \cong P$ in $\mathcal{F}$, then $\operatorname{Rep}_{\mathcal{F}}(Q, P)$ consists of precisely one free orbit of $\Gamma$, so $m=1, N_{S}^{\Gamma_{1}^{\prime}}(Q)=Q$, and $\beta^{*}\left(\alpha^{*} \Phi\right)(P) \cong \Phi(Q)$.

Proposition 3.2 does in fact still hold for functors from $\mathcal{O}^{c}(\mathcal{F})$ to abelian groups (not just $p$-locally) — if one defines $\Lambda^{*}(\Gamma ; M)$ in this generality, and the prime $p$ is understood.

We next list some easy consequences of Proposition 3.2 
Definition 3.3. A category $\mathcal{C}$ has bounded limits at $p$ if there is an integer $d$ such that for every functor $\Phi: \mathcal{C}^{\text {op }} \longrightarrow \mathbb{Z}_{(p)}$-mod we have $\varliminf^{i}(\Phi)=0$ for $i>d$.

The following corollary will be needed in the next section.

Corollary 3.4. Let $\mathcal{F}$ be any saturated fusion system. Then the $\mathcal{F}$-centric orbit category $\mathcal{O}^{c}(\mathcal{F})$ has bounded limits at $p$.

Proof. By [JMO2, Proposition 4.11], for any finite group $\Gamma$, there is some $k_{\Gamma}$ such that $\Lambda^{i}(\Gamma ; M)=0$ for all $\mathbb{Z}_{(p)}[\Gamma]$-modules $M$ and all $i>k_{\Gamma}$. Let $k$ be the maximum of the $k_{\mathrm{Out}_{\mathcal{F}}(P)}$ for all $\mathcal{F}$-centric $P \leq S$. Then by Proposition 3.2, for each functor $\Phi: \mathcal{O}^{c}(\mathcal{F})^{\text {op }} \longrightarrow \mathbb{Z}_{(p)}$-mod which vanishes except on one orbit type, $\varliminf^{i}(\Phi)=0$ for $i>k$. The same result for an arbitrary $p$-local functor $\Phi$ on $\mathcal{O}^{c}(\mathcal{F}) \overleftarrow{\text { now follows }}$ from the exact sequences of higher limits associated to short exact sequences of functors.

Another consequence of Proposition 3.2 is

Corollary 3.5. Let $\mathcal{F}$ be any saturated fusion system over a $p$-group $S$. If $\operatorname{rk}_{p}(S)<$ $p^{3}$, then there exists a centric linking system associated to $\mathcal{F}$. If $\operatorname{rk}_{p}(S)<p^{2}$, then there exists a unique centric linking system associated to $\mathcal{F}$.

Proof. By [BLO, Proposition 5.8] (which is based on the work of Grodal [Gr]), for any finite group $\Gamma$ and any finite $\mathbb{Z}_{(p)}[\Gamma]$-module $M, \Lambda^{i}(\Gamma, M)=0$ if $\operatorname{rk}(M)<p^{i}$. So if $\operatorname{rk}_{p}(S)<p^{3}$, then $\Lambda^{3}(N(P) / P ; Z(P))=0$ for all $\mathcal{F}$-centric $P \leq S$, and hence $\lim ^{3}\left(\mathcal{Z}_{\mathcal{F}}\right)=0$ by Proposition 3.2 By the same argument, $\varliminf^{2}\left(\mathcal{Z}_{\mathcal{F}}\right)=0$ if $\mathrm{rk}_{p}(S)<p^{2}$. The result now follows from Proposition 3.1.

If $\mathcal{F}$ is a fusion system over $S$, then a subgroup $P \leq S$ is called $\mathcal{F}$-radical if $\operatorname{Out}_{\mathcal{F}}(P)$ contains no nontrivial normal $p$-subgroup (see Definition A.9). As another application of Proposition 3.2, we show that we can remove from a centric linking system certain subgroups which are not $\mathcal{F}$-radical, without changing the mod $p$ homology type of its nerve. In fact, the following lemma also holds without the assumption that overgroups of subgroups in $\mathcal{L}_{0}$ are also in $\mathcal{L}_{0}$, but this assumption does simplify the proof, and suffices for the purposes of this paper.

Corollary 3.6. Fix a p-local finite group $(S, \mathcal{F}, \mathcal{L})$. Let $\mathcal{L}_{0} \subseteq \mathcal{L}$ be a full subcategory which contains all $\mathcal{F}$-radical $\mathcal{F}$-centric subgroups of $S$. Assume also that if $P \leq$ $P^{\prime} \leq S$ and $P \in \operatorname{Ob}\left(\mathcal{L}_{0}\right)$, then $P^{\prime} \in \operatorname{Ob}\left(\mathcal{L}_{0}\right)$. Then the inclusion $\left|\mathcal{L}_{0}\right| \subseteq|\mathcal{L}|$ is a mod $p$ homology equivalence.

Proof. Let $\mathcal{F}_{0} \subseteq \mathcal{F}^{c}$ be the full subcategory with $\operatorname{Ob}\left(\mathcal{F}_{0}\right)=\operatorname{Ob}\left(\mathcal{L}_{0}\right)$. By Proposition 2.2, there are homotopy decompositions

$$
|\mathcal{L}| \simeq \underset{\mathcal{O}^{c}(\mathcal{F})}{\operatorname{hocolim}}(\widetilde{B}) \quad \text { and } \quad\left|\mathcal{L}_{0}\right| \simeq \underset{\mathcal{O}\left(\mathcal{F}_{0}\right)}{\operatorname{hocolim}}(\widetilde{B})
$$

which give rise to spectral sequences

$$
E_{2}^{i j}(\mathcal{L})=\underset{\mathcal{O}^{c}(\mathcal{F})}{\lim ^{i}}\left(H^{j}(-)\right) \Longrightarrow H^{i+j}(|\mathcal{L}|)
$$

and

$$
E_{2}^{i j}\left(\mathcal{L}_{0}\right)=\varliminf_{\mathcal{O}\left(\mathcal{F}_{0}\right)}^{i}\left(H^{j}(-)\right) \Longrightarrow H^{i+j}\left(\left|\mathcal{L}_{0}\right|\right)
$$


For each $P \in \operatorname{Ob}\left(\mathcal{F}^{c}\right) \backslash \mathrm{Ob}\left(\mathcal{F}_{0}\right), P$ is not $\mathcal{F}$-radical by assumption, so $\operatorname{Out}_{\mathcal{F}}(P)$ contains a nontrivial normal $p$-subgroup. So [JMO, Proposition 6.1(ii)] applies to show that $\Lambda^{*}\left(\operatorname{Out}_{\mathcal{F}}(P) ; M\right)=0$ for all $\mathbb{Z}_{(p)}\left[\operatorname{Out}_{\mathcal{F}}(P)\right]$-modules $M$. Hence by Proposition 3.2 (and since any overgroup of an object in $\mathcal{F}_{0}$ is also in $\mathcal{F}_{0}$ ), for any functor $F: \mathcal{O}^{c}(\mathcal{F}) \longrightarrow \mathbb{Z}_{(p)}$-mod, $\varliminf^{*}(F)$ is the same over $\mathcal{O}^{c}(\mathcal{F})$ and over $\mathcal{O}\left(\mathcal{F}_{0}\right)$. The inclusion of the above spectral sequences thus induces an isomorphism $E_{2}\left(\mathcal{L}_{0}\right) \cong E_{2}(\mathcal{L})$. So the inclusion of $\left|\mathcal{L}_{0}\right|$ in $|\mathcal{L}|$ is a $\bmod p$ equivalence, and induces a homotopy equivalence $\left|\mathcal{L}_{0}\right|_{p}^{\wedge} \simeq|\mathcal{L}|_{p}^{\wedge}$.

\section{Spaces of MAPS}

We next study the mapping $\operatorname{spaces} \operatorname{Map}\left(B Q,|\mathcal{L}|_{p}^{\wedge}\right)$, where $|\mathcal{L}|_{p}^{\wedge}$ is the classifying space of a $p$-local finite group $(S, \mathcal{F}, \mathcal{L})$. We will see in Theorem 4.4 and Corollary 4.5 that the set $\left[B Q,|\mathcal{L}|_{p}^{\wedge}\right]$ of homotopy classes is described in terms of conjugacy classes of homomorphisms, analogously to the case for maps to $p$-completed classifying spaces of finite groups. We also describe the individual connected components of these mapping spaces in certain cases, but a complete description will have to wait until Section 6

Throughout this section, $H^{*}(-)$ denotes cohomology with coefficients in $\mathbb{F}_{p}$. We first show that under certain conditions, mapping spaces $\operatorname{Map}(B Q,-)$ "commute" with homotopy colimits. A similar result was shown by the first author and Nitu Kitchloo in [BrK Theorem 6.11]. Recall the definition of "bounded limits at $p$ " (Definition 3.3).

Lemma 4.1. Fix a prime $p$, and let $V$ be a group of order $p$. Let $\mathcal{C}$ be a finite category with bounded limits at $p$, and let

$$
F: \mathcal{C} \longrightarrow V \text {-Spaces }
$$

be a functor such that for each $c$ in $\mathcal{C}, F(c)$ and $F(c)^{h V}$ are both p-complete and have finite mod $p$ cohomology in each degree. Then the natural map

$$
\left[\underset{\mathcal{C}}{\operatorname{hocolim}}\left(F(-)^{h V}\right)\right]_{p}^{\wedge} \longrightarrow\left[(\underset{\mathcal{C}}{\operatorname{hocolim}}(F))_{p}^{\wedge}\right]^{h V}
$$

is a homotopy equivalence.

Proof. To simplify the notation, set

$$
X=\underset{\mathcal{C}}{\operatorname{hocolim}}(F) \quad \text { and } \quad Z=\underset{\mathcal{C}}{\stackrel{\operatorname{hocolim}}{\longrightarrow}}\left(F(-)^{h V}\right) .
$$

Write $X=\bigcup_{i=0}^{\infty} X_{i}$ and $Z=\bigcup_{i=0}^{\infty} Z_{i}$, where $X_{i}$ and $Z_{i}$ are the "skeleta" of the homotopy colimits.

We first recall the notation of Lannes [La, §4]. For any $M$ in $H^{*} V$ - $\mathcal{U}$, i.e., any unstable module over the Steenrod algebra with compatible $H^{*} V$-module structure, $T_{V}(M)$ is a $T_{V}\left(H^{*} V\right)$-module, and $T_{V}\left(H^{*} V\right) \cong \prod_{\mathrm{Hom}(V, V)} H^{*} V$. Lannes defines

$$
\operatorname{Fix}(M)=\mathbb{F}_{p}((1)) \otimes_{T_{V}\left(H^{*} V\right)} T_{V}(M),
$$

where $\mathbb{F}_{p}((1))$ is the factor $\mathbb{F}_{p}$ corresponding to $\operatorname{Id} \in \operatorname{Hom}(V, V)$ (regarded as a quotient algebra). For any $V$-space $Y$, Lannes defines $H^{V *}(Y)=\operatorname{Fix}\left(H_{V}^{*}(Y)\right)$, where $H_{V}^{*}(Y)=H^{*}\left(E V \times_{V} Y\right)$. The goal of [La, $\left.\S 4\right]$ is to find conditions under which $H^{V *}(Y) \cong H^{*}\left(\left(Y_{p}^{\wedge}\right)^{h V}\right)$. By [La Theorem 4.6.1.1], Fix is an exact functor. 
Consider the following exact couples:
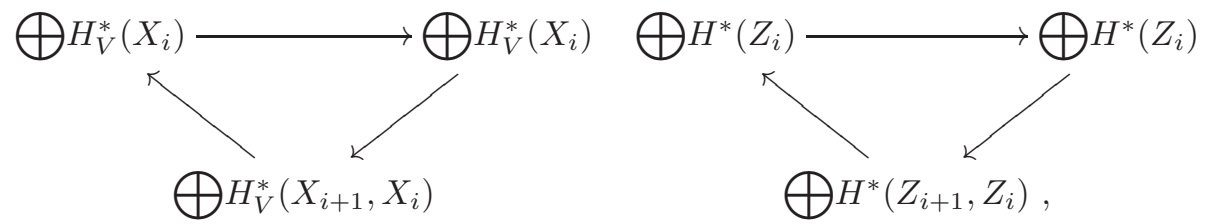

and let $E_{r}^{* *}\left(X_{h V}\right)$ and $E_{r}^{* *}(Z)$ denote the induced spectral sequences. Then $E_{r}^{* *}\left(X_{h V}\right)$ is a spectral sequence of modules in $H^{*} V-\mathcal{U}$, in the sense that each column of each page $E_{r}^{* *}\left(X_{h V}\right)$ as well as the differentials $d_{r}$ are in $H^{*} V-\mathcal{U}$. The equivariant maps

$$
E V \times Z_{i} \longrightarrow X_{i}
$$

(where $V$ acts trivially on $Z_{i}$ ) induce via adjointness applied to the exact couples a homomorphism of spectral sequences

$$
T_{V}\left(E_{r}^{* *}\left(X_{h V}\right)\right) \longrightarrow E_{r}^{* *}(Z),
$$

where $T_{V}$ is applied to each column in each page of the spectral sequence. After tensoring over $T_{V}\left(H^{*} V\right)$ with $\mathbb{F}_{p}((1))$, this map induces a homomorphism of spectral sequences

$$
\Phi: \operatorname{Fix}\left(E_{r}^{* *}\left(X_{h V}\right)\right) \longrightarrow E_{r}^{* *}(Z) .
$$

We first consider the case $r=2$. For each object $c$ in $\mathcal{C}$, the spaces $F(c)$ and $F(c)^{h V}$ are $p$-complete by assumption. Hence $H^{*}\left(F(c)^{h V}\right) \cong \operatorname{Fix}\left(H_{V}^{*}(F(c))\right)$ by [La] Theorem 4.9.1] (applied with $Z=F(c)^{h V}$ and $X=F(c)$ ). Thus

$$
E_{2}^{j *}\left(X_{h V}\right) \cong \varlimsup_{\mathcal{C}}^{\lim ^{j}}\left(H_{V}^{*}(F(-))\right) \quad \text { and } \quad E_{2}^{j *}(Z) \cong \varlimsup_{\mathcal{C}}^{\lim ^{j}}\left(\operatorname{Fix}\left(H_{V}^{*}(F(-))\right)\right),
$$

and $\Phi: \operatorname{Fix}\left(E_{2}^{* *}\left(X_{h V}\right)\right) \longrightarrow E_{2}^{* *}(Z)$ is the natural map. Since Fix is exact and commutes with finite products, and since $\mathcal{C}$ is a finite category, we have

$$
\left.{\underset{\mathcal{C}}{\lim ^{j}}}^{j} \operatorname{Fix}\left(H_{V}^{*}(F(-))\right)\right) \cong \operatorname{Fix}\left(\varliminf_{\mathcal{C}}\left(H_{V}^{*}(F(-))\right)\right)
$$

for each $j$. It follows that $\Phi$ is an isomorphism when $r=2$.

Thus $\Phi$ is also an isomorphism when $r=\infty$. Since $\mathcal{C}$ has bounded limits at $p$ by assumption, there are only a finite number of nonzero columns in each spectral sequence, and so the resulting filtrations of $H^{*}(Z)$ and $\operatorname{Fix}\left(H_{V}^{*}(X)\right)$ are both finite. Hence (using the exactness of Fix again) $\Phi$ induces an isomorphism

$$
\operatorname{Fix}\left(H_{V}^{*}(X)\right) \longrightarrow H^{*}(Z) \text {. }
$$

By [La, Theorem 4.9.1] again, this implies that

$$
Z_{p}^{\wedge} \longrightarrow\left(X_{p}^{\wedge}\right)^{h V}
$$

is a homotopy equivalence, which is what we wanted to show.

This will now be applied to describe maps into a homotopy colimit in certain cases.

Proposition 4.2. Fix a prime $p$ and a p-group $Q$. Let $\mathcal{C}$ be a finite category with bounded limits at $p$, and let

$$
F: \mathcal{C} \longrightarrow \text { Top }
$$


be a functor such that for each $c$ in $\mathcal{C}$ and each $Q_{0} \leq Q, \operatorname{Map}\left(B Q_{0}, F(c)\right)$ is $p$ complete and has finite mod $p$ cohomology in each degree. Then the natural map

$$
[\underset{\mathcal{C}}{\operatorname{hocolim}}(\operatorname{Map}(B Q, F))]_{p}^{\wedge} \longrightarrow \operatorname{Map}\left(B Q,(\underset{\mathcal{C}}{\operatorname{\text {hocolim}}} F)_{p}^{\wedge}\right)
$$

is a homotopy equivalence. Here, $\operatorname{Map}(B Q, F)$ denotes the functor which sends $c$ to $\operatorname{Map}(B Q, F(c))$.

Proof. We prove this by induction on $|Q|$; the result is clear when $|Q|=1$. So assume $Q \neq 1$, let $Q_{0} \triangleleft Q$ be a normal subgroup of index $p$, and set $V=Q / Q_{0}$.

By the induction hypothesis, the map

$$
\left[\underset{\mathcal{C}}{\operatorname{hocolim}}\left(\operatorname{Map}\left(E Q / Q_{0}, F\right)\right)\right]_{p}^{\wedge} \simeq \operatorname{Map}\left(E Q / Q_{0},(\underset{\mathcal{C}}{(\operatorname{hocolim}} F)_{p}^{\wedge}\right)
$$

is a homotopy equivalence. It is also equivariant with respect to the $V$-actions induced by the action of $V$ on $E Q / Q_{0}$, and hence induces a homotopy equivalence

$$
\begin{aligned}
\left(\left[\underset{\mathcal{C}}{\operatorname{hocolim}}\left(\operatorname{Map}\left(E Q / Q_{0}, F\right)\right)\right]_{p}^{\wedge}\right)^{h V} & \stackrel{\simeq}{\longrightarrow}\left(\operatorname{Map}\left(E Q / Q_{0},(\underset{\mathcal{C}}{\operatorname{\text {hocolim}}} F)_{p}^{\wedge}\right)\right)^{h V} \\
& \simeq \operatorname{Map}\left(B Q,(\underset{\mathcal{C}}{\operatorname{hocolim}} F)_{p}^{\wedge}\right) .
\end{aligned}
$$

Furthermore, by assumption, the mapping spaces

$$
\operatorname{Map}\left(B Q_{0}, F(c)\right) \quad \text { and } \quad\left(\operatorname{Map}\left(B Q_{0}, F(c)\right)\right)^{h V} \simeq \operatorname{Map}(B Q, F(c))
$$

are $p$-complete for each $c \in \mathrm{Ob}(\mathcal{C})$, and Proposition 4.1 applies to show that

$$
[\underset{\mathcal{C}}{\operatorname{hocolim}}(\operatorname{Map}(B Q, F))]_{p}^{\wedge} \simeq\left(\left[\underset{\mathcal{C}}{\operatorname{hocolim}}\left(\operatorname{Map}\left(E Q / Q_{0}, F\right)\right)\right]_{p}^{\wedge}\right)^{h V}
$$

is a homotopy equivalence. The proposition now follows from (1) and (2).

We now apply this to spaces of maps to the classifying space of a $p$-local finite group.

Proposition 4.3. Fix a p-local finite group $(S, \mathcal{F}, \mathcal{L})$ and a finite $p$-group $Q$. Let $\mathcal{F}_{0} \subseteq \mathcal{F}^{c}$ be any full subcategory, and let $\mathcal{L}_{0} \subseteq \mathcal{L}$ be the full subcategory with $\operatorname{Ob}\left(\mathcal{L}_{0}\right)=\operatorname{Ob}\left(\mathcal{F}_{0}\right)$. Let $\left(\mathcal{L}_{0}\right)_{Q}$ be the category whose objects are the pairs $(P, \alpha)$ for $P \in \operatorname{Ob}\left(\mathcal{L}_{0}\right)$ and $\alpha \in \operatorname{Hom}(Q, P)$, and where

$$
\operatorname{Mor}_{\left(\mathcal{L}_{0}\right)_{Q}}\left((P, \alpha),\left(P^{\prime}, \alpha^{\prime}\right)\right)=\left\{\varphi \in \operatorname{Mor}_{\mathcal{L}_{0}}\left(P, P^{\prime}\right) \mid \alpha^{\prime}=\pi(\varphi) \circ \alpha \in \operatorname{Hom}\left(Q, P^{\prime}\right)\right\} .
$$

Let $\Phi:\left(\mathcal{L}_{0}\right)_{Q} \times \mathcal{B}(Q) \longrightarrow \mathcal{L}_{0}$ be the functor defined by setting

$$
\Phi\left((P, \alpha), o_{Q}\right)=P \quad \text { and } \quad \Phi\left((P, \alpha) \stackrel{\varphi}{\longrightarrow}\left(P^{\prime}, \alpha^{\prime}\right), \check{x}\right)=\varphi \circ \widehat{\alpha(x)} .
$$

Then the map

$$
|\Phi|^{\prime}:\left|\left(\mathcal{L}_{0}\right)_{Q}\right|_{p}^{\wedge} \longrightarrow \operatorname{Map}\left(B Q,\left|\mathcal{L}_{0}\right|_{p}^{\wedge}\right)
$$

adjoint to $|\Phi|$ is a homotopy equivalence.

Proof. Note first that $\Phi(\varphi, \check{x})=\varphi \circ \widehat{\alpha(x)}=\widehat{\alpha^{\prime}(x)} \circ \varphi$ by condition $(\mathrm{C})$. Thus $\Phi$ is a well defined functor.

Let $\mathcal{O}_{0} \subseteq \mathcal{O}^{c}(\mathcal{F})$ be the full subcategory with $\operatorname{Ob}\left(\mathcal{O}_{0}\right)=\operatorname{Ob}\left(\mathcal{F}_{0}\right)=\operatorname{Ob}\left(\mathcal{L}_{0}\right)$, and let $\tilde{\pi}: \mathcal{L}_{0} \longrightarrow \mathcal{O}_{0}$ be the projection functor. Let $\widetilde{\pi}_{Q}:\left(\mathcal{L}_{0}\right)_{Q} \longrightarrow \mathcal{O}_{0}$ be the functor $\tilde{\pi}_{Q}(P, \alpha)=P$ and $\widetilde{\pi}_{Q}(\varphi)=\widetilde{\pi}(\varphi)$. Let

$$
\widetilde{B}_{Q}, \widetilde{B}: \mathcal{O}_{0} \longrightarrow \text { Top }
$$


be the left homotopy Kan extensions over $\widetilde{\pi}_{Q}$ and $\widetilde{\pi}$, respectively, of the constant functors $*$. Then

$$
\left|\mathcal{L}_{0}\right| \simeq \underset{\mathcal{O}_{0}}{\operatorname{hocolim}}(\widetilde{B}) \quad \text { and } \quad\left|\left(\mathcal{L}_{0}\right)_{Q}\right| \simeq \underset{\mathcal{O}_{0}}{\operatorname{hocolim}}\left(\widetilde{B}_{Q}\right)
$$

(cf. [HV, Theorem 5.5]).

Consider the commutative triangle

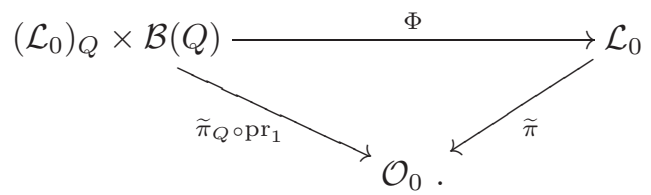

The left homotopy Kan extension over $\widetilde{\pi}_{Q} \circ \mathrm{pr}_{1}$ of the constant functor $*$ is the functor $\widetilde{B}_{Q}(-) \times B Q$, and so the triangle induces a natural transformation of functors

$$
\Phi^{\prime}: \widetilde{B}_{Q}(-) \times B Q \longrightarrow \widetilde{B} .
$$

The adjoint map $\widetilde{\Phi}: \widetilde{B}_{Q} \longrightarrow \operatorname{Map}(B Q, \widetilde{B})$ to $\Phi^{\prime}$ is also a natural transformation of functors from $\mathcal{O}_{0}$ to Top, and induces a commutative diagram

$$
\begin{gathered}
\left.\underset{\mathcal{O}_{0}}{(\operatorname{hocolim}}\left(\widetilde{B}_{Q}\right)\right)_{p}^{\wedge} \stackrel{\text { hocolim }(\widetilde{\Phi})}{\longrightarrow}\left(\underset{\mathcal{O}_{0}}{\operatorname{hocolim}} \operatorname{Map}(B Q, \widetilde{B})\right)_{p}^{\wedge} \stackrel{\omega}{\simeq} \operatorname{Map}\left(B Q, \underset{\mathcal{O}_{0}}{\longrightarrow} \operatorname{hocolim}(\widetilde{B})_{p}^{\wedge}\right) \\
\quad \simeq \downarrow \\
\left|\left(\mathcal{L}_{0}\right)_{Q}\right|_{p}^{\wedge} \\
\operatorname{Map}\left(B Q,\left|\mathcal{L}_{0}\right|_{p}^{\wedge}\right) .
\end{gathered}
$$

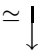

For each $P \leq S$ and $Q_{0} \leq Q$, each component of $\operatorname{Map}\left(B Q_{0}, B P\right)$ is of the form $B C_{P}\left(\rho\left(Q_{0}\right)\right)$ for some $\rho \in \operatorname{Hom}\left(Q_{0}, P\right)$. So all such mapping spaces are $p$-complete and have finite mod $p$ cohomology in each degree, and hence $\omega$ is a homotopy equivalence by Proposition 4.2. It remains only to show that $\widetilde{\Phi}(P)$ is a homotopy equivalence for each $P$.

For each $P$ in $\mathcal{O}_{0}, \widetilde{B}(P)$ is the nerve of the overcategory $\widetilde{\pi} \downarrow P$, whose objects are the pairs $(R, \chi)$ for $R \in \operatorname{Ob}\left(\mathcal{L}_{0}\right)=\operatorname{Ob}\left(\mathcal{O}_{0}\right)$ and $\chi \in \operatorname{Rep}_{\mathcal{F}}(R, P)$, and where

$$
\operatorname{Mor}_{\tilde{\pi} \downarrow P}\left((R, \chi),\left(R^{\prime}, \chi^{\prime}\right)\right)=\left\{\varphi \in \operatorname{Mor}_{\mathcal{L}_{0}}\left(R, R^{\prime}\right) \mid \chi=\chi^{\prime} \circ \widetilde{\pi}(\varphi)\right\} .
$$

Let $\mathcal{B}^{\prime}(P)$ be the full subcategory of $\widetilde{\pi} \downarrow P$ with the unique object $(P, \mathrm{Id})$, and with morphisms the group of all $\widehat{g}$ for $g \in P$.

Similarly, $\widetilde{B}_{Q}(P)$ is the nerve of the category $\widetilde{\pi}_{Q} \downarrow P$, whose objects are the triples $(R, \alpha, \chi)$ for $R \in \operatorname{Ob}\left(\mathcal{L}_{0}\right)=\operatorname{Ob}\left(\mathcal{O}_{0}\right), \alpha \in \operatorname{Hom}(Q, R)$, and $\chi \in \operatorname{Rep}_{\mathcal{F}}(R, P)$; and where

$\operatorname{Mor}_{\tilde{\pi}_{Q \downarrow} \downarrow P}\left((R, \alpha, \chi),\left(R^{\prime}, \alpha^{\prime}, \chi^{\prime}\right)\right)=\left\{\varphi \in \operatorname{Mor}_{\mathcal{L}_{0}}\left(R, R^{\prime}\right) \mid \alpha^{\prime}=\pi(\varphi) \circ \alpha, \chi=\chi^{\prime} \circ \widetilde{\pi}(\varphi)\right\}$.

Let $\mathcal{B}_{Q}^{\prime}(P)$ be the full subcategory of $\tilde{\pi}_{Q} \downarrow P$ with objects the triples $(P, \alpha$, Id) for $\alpha \in \operatorname{Hom}(Q, P)$.

Fix a section $\widetilde{\sigma}: \operatorname{Mor}\left(\mathcal{O}_{0}\right) \longrightarrow \operatorname{Mor}\left(\mathcal{L}_{0}\right)$ which sends identity morphisms to identity morphisms. Retractions

$$
\tilde{\pi} \downarrow P \stackrel{\Psi}{\longrightarrow} \mathcal{B}^{\prime}(P) \quad \text { and } \quad \tilde{\pi}_{Q} \downarrow P \stackrel{\Psi_{Q}}{\longrightarrow} \mathcal{B}_{Q}^{\prime}(P)
$$

are defined by setting

$$
\Psi(R, \chi)=(P, \mathrm{Id}) \quad \text { and } \quad \Psi_{Q}(R, \alpha, \chi)=(P, \pi \widetilde{\sigma}(\chi) \circ \alpha, \mathrm{Id}),
$$


and by sending $\varphi$ in $\operatorname{Mor}_{\tilde{\pi} \downarrow P}\left((R, \chi),\left(R^{\prime}, \chi^{\prime}\right)\right)$ or $\operatorname{Mor}_{\tilde{\pi}_{Q} \downarrow P}\left((R, \alpha, \chi),\left(R^{\prime}, \alpha^{\prime}, \chi^{\prime}\right)\right)$ to $\widehat{g} \in \operatorname{Aut}_{\mathcal{L}_{0}}(P)$, where $g \in P$ is the unique element such that $\widetilde{\sigma}\left(\chi^{\prime}\right) \circ \varphi=\widehat{g} \circ \tilde{\sigma}(\chi)$ in $\operatorname{Mor}_{\mathcal{L}_{0}}(R, P)$ (Lemma 1.10(b)). There are natural transformations

$$
\operatorname{Id}_{\tilde{\pi} \downarrow P} \longrightarrow \operatorname{incl} \circ \Psi \quad \text { and } \quad \operatorname{Id}_{\tilde{\pi}_{Q \downarrow} \downarrow P} \longrightarrow \operatorname{incl} \circ \Psi_{Q}
$$

of functors which send an object $(R, \chi)$ to $\chi \in \operatorname{Mor}_{\tilde{\pi} \downarrow P}((R, \chi),(P, \operatorname{Id}))$ and similarly for an object $(R, \alpha, \chi)$. This shows that $\left|\mathcal{B}^{\prime}(P)\right| \subseteq|\widetilde{\pi} \downarrow P|$ and $\left|\mathcal{B}_{Q}^{\prime}(P)\right| \subseteq\left|\tilde{\pi}_{Q} \downarrow P\right|$ are deformation retracts.

It remains to show for each $P$ that $\widetilde{\Phi}(P)$ restricts to a homotopy equivalence

$$
\widetilde{\Phi}_{0}(P):\left|\mathcal{B}_{Q}^{\prime}(P)\right| \longrightarrow \operatorname{Map}\left(B Q,\left|\mathcal{B}^{\prime}(P)\right|\right) .
$$

Two objects $(P, \alpha, \operatorname{Id})$ and $\left(P, \alpha^{\prime}, \mathrm{Id}\right)$ in $\mathcal{B}_{Q}^{\prime}(P)$ are isomorphic if and only if $\alpha$ and $\alpha^{\prime}$ are conjugate in $P$, and the automorphism group of $(P, \alpha, \mathrm{Id})$ is isomorphic to $C_{P}(\alpha Q)$. This shows that

$$
\widetilde{B}_{Q}(P) \simeq \coprod_{\alpha \in \operatorname{Rep}(Q, P)} B C_{P}(\alpha Q) .
$$

Since $\left|\mathcal{B}^{\prime}(P)\right| \cong B P$, and since $\widetilde{\Phi}^{\prime}$ is induced by the homomorphisms (incl $\cdot \alpha$ ) from $C_{P}(\alpha Q) \times Q$ to $P$, it follows that (1) is an equivalence.

We now apply Proposition 4.3 to describe more explicitly the set $\left[B Q,|\mathcal{L}|_{p}^{\wedge}\right]$ of homotopy classes of maps, as well as the individual components in certain cases. Later, in Theorem 6.3 we show that all components of $\operatorname{Map}\left(B Q,|\mathcal{L}|_{p}^{\wedge}\right)$ can be described in terms of centralizers, in a way analogous to the description of components in $\operatorname{Map}\left(B Q, B G_{p}^{\wedge}\right)$.

Theorem 4.4. Let $(S, \mathcal{F}, \mathcal{L})$ be a p-local finite group, and let $f: B S \longrightarrow|\mathcal{L}|_{p}^{\wedge}$ be the natural inclusion followed by completion. Then the following hold, for any p-group $Q$.

(a) Each map $B Q \longrightarrow|\mathcal{L}|_{p}^{\wedge}$ is homotopic to $f \circ B \rho$ for some $\rho \in \operatorname{Hom}(Q, S)$.

(b) Given any two homomorphisms $\rho, \rho^{\prime} \in \operatorname{Hom}(Q, S), f \circ B \rho \simeq f \circ B \rho^{\prime}$ as maps from $B Q$ to $|\mathcal{L}|_{p}^{\wedge}$ if and only if there is some $\chi \in \operatorname{Hom}_{\mathcal{F}}\left(\rho Q, \rho^{\prime} Q\right)$ such that $\rho^{\prime}=\chi \circ \rho$.

(c) For each $\rho \in \operatorname{Hom}(Q, S)$ such that $\rho Q$ is $\mathcal{F}$-centric, the composite

$$
B Z(\rho Q) \times B Q \stackrel{\text { incl } \cdot B \rho}{\longrightarrow} B S \stackrel{f}{\longrightarrow}|\mathcal{L}|_{p}^{\wedge}
$$

induces a homotopy equivalence

$$
B Z(\rho Q) \stackrel{\simeq}{\longrightarrow} \operatorname{Map}\left(B Q,|\mathcal{L}|_{p}^{\wedge}\right)_{f \circ B \rho} .
$$

(d) The evaluation map induces a homotopy equivalence

$$
\operatorname{Map}\left(B Q,|\mathcal{L}|_{p}^{\wedge}\right)_{\text {triv }} \simeq|\mathcal{L}|_{p}^{\wedge}
$$

Proof. We refer to the category $\mathcal{L}_{Q}$, and to the homotopy equivalence

$$
|\Phi|^{\prime}:\left|\mathcal{L}_{Q}\right|_{p}^{\wedge} \simeq \operatorname{Map}\left(B Q,|\mathcal{L}|_{p}^{\wedge}\right)
$$

of Proposition 4.3.

By definition, $|\Phi|^{\prime}$ sends a vertex $(P, \alpha)$ to the morphism $B \alpha$, and two vertices $(P, \alpha)$ and $\left(P^{\prime}, \alpha^{\prime}\right)$ are in the same connected component of $\left|\mathcal{L}_{Q}\right|$ if and only if 
there is some $\chi \in \operatorname{Hom}_{\mathcal{F}}\left(\alpha Q, \alpha^{\prime} Q\right)$ such that $\alpha^{\prime}=\chi \circ \alpha$. Points (a) and (b) now follow immediately. If $\rho \in \operatorname{Hom}(Q, S)$ is such that $\rho Q$ is $\mathcal{F}$-centric, then the connected component of $\left|\mathcal{L}_{Q}\right|$ which contains the vertex $(\rho Q, \rho)$ contains as deformation retract the nerve of the full subcategory with that as its only object. Since $\operatorname{Aut}_{\mathcal{L}_{Q}}(\rho Q, \rho) \cong Z(\rho Q)$, this component has the homotopy type of $B Z(\rho Q)$, which proves point (c). Point (d) holds since the component of $\mathcal{L}_{Q}$ which contains the objects $(P, 1)$ is equivalent to $\mathcal{L}$.

If $(S, \mathcal{F}, \mathcal{L})$ is a $p$-local finite group, then for any finite $p$-group $Q$ we define

$$
\operatorname{Rep}(Q, \mathcal{L})=\operatorname{Hom}(Q, S) / \sim,
$$

where $\sim$ is the equivalence relation defined by setting $\rho \sim \rho^{\prime}$ if there is some $\chi \in \operatorname{Hom}_{\mathcal{F}}\left(\rho Q, \rho^{\prime} Q\right)$ such that $\rho^{\prime}=\chi \circ \rho$. Theorem 4.4(a,b) can now be restated as follows.

Corollary 4.5. Fix a p-local finite group $(S, \mathcal{F}, \mathcal{L})$, and let $f: B S \longrightarrow|\mathcal{L}|_{p}^{\wedge}$ be the natural inclusion followed by completion. Then the map

$$
\operatorname{Rep}(Q, \mathcal{L}) \stackrel{\cong}{\longrightarrow}\left[B Q,|\mathcal{L}|_{p}^{\wedge}\right],
$$

defined by sending the class of $\rho: Q \longrightarrow S$ to $f \circ B \rho$, is a bijection.

\section{The cohomology Ring of a $p$-LOcal finite group}

Throughout this section, all cohomology is taken with coefficients in $\mathbb{F}_{p}$. Since $|\mathcal{L}|$ is $p$-good for any $p$-local finite group $(S, \mathcal{F}, \mathcal{L})$ by Proposition 1.12, $H^{*}\left(|\mathcal{L}|_{p}^{\wedge}\right) \cong$ $H^{*}(|\mathcal{L}|)$.

For any fusion system $\mathcal{F}$ over a $p$-group $S$, we write

$$
H^{*}(\mathcal{F})=\lim _{\overleftarrow{\mathcal{O}(\mathcal{F})}} H^{*}(-)
$$

to denote the "ring of stable elements for $\mathcal{F}$ ", regarded as a subring of $H^{*}(B S)$. We think of this as the cohomology of the fusion system $\mathcal{F}$. If $\mathcal{F}$ is saturated, then

$$
H^{*}(\mathcal{F})=\lim _{\overleftarrow{\mathcal{O}(\mathcal{F})}} H^{*}(-) \cong \lim _{\overleftarrow{\mathcal{O}^{c}(\mathcal{F})}} H^{*}(-)
$$

by Theorem A.10 (Alperin's fusion theorem for fusion systems). The main results of this section are that for any $p$-local finite group $(S, \mathcal{F}, \mathcal{L})$ the natural map

$$
R_{\mathcal{L}}: H^{*}(|\mathcal{L}|) \longrightarrow H^{*}(\mathcal{F})
$$

is an isomorphism, and that this cohomology ring is noetherian. These generalize well known, classical results on $H^{*}(B G)$ when $G$ is a finite group.

We first show (Proposition 5.2) that $H^{*}(\mathcal{F})$ is noetherian for any fusion system $\mathcal{F}$. This is implicit in a paper of Evens and Priddy [EP], but since it is not stated explicitly there, we give our interpretation of their proof here.

For any $p$-group $S$, let $\mathcal{E}(S)$ be the set of elementary abelian subgroups of $S$, and let $\mathcal{F}_{S}^{e} \subseteq \mathcal{F}_{S}(S)$ be the full subcategory whose object set is $\mathcal{E}(S)$. More generally, if $\mathcal{F}$ is any fusion system over $S$, then $\mathcal{F}^{e} \subseteq \mathcal{F}$ denotes the full subcategory with object set $\mathcal{E}(S)$. Set

$$
H_{\mathcal{E}}^{*}(\mathcal{F})=\lim _{\mathcal{F}^{e}} H^{*}(-) \quad \text { and } \quad H_{\mathcal{E}}^{*}(S)={\underset{\mathcal{F}}{\mathcal{F}_{S}^{e}}}_{m} H^{*}(-)
$$

These are both regarded as subrings of the product of the $H^{*}(B E)$ for $E \in \mathcal{E}(S)$. 
Recall $[\mathrm{Qu}$ that a ring homomorphism $\gamma: B \longrightarrow A$ is called an $F$-monomorphism if each element $b \in \operatorname{Ker}(\gamma)$ is nilpotent, an $F$-epimorphism if for all $a \in A$ there is some $k>0$ such that $a^{k} \in \operatorname{Im}(\gamma)$, and an $F$-isomorphism if it is both an $F$-monomorphism and an $F$-epimorphism.

Proposition 5.1. For any fusion system $\mathcal{F}$ over a p-group $S$, restriction to elementary abelian subgroups defines $F$-isomorphisms

$$
\lambda_{S}: H^{*}(B S) \longrightarrow H_{\mathcal{E}}^{*}(S) \quad \text { and } \quad \lambda_{\mathcal{F}}: H^{*}(\mathcal{F}) \longrightarrow H_{\mathcal{E}}^{*}(\mathcal{F}) .
$$

Proof. The homomorphism $\lambda_{S}$ is an $F$-isomorphism by $\mathrm{Qu}$, Theorem 6.2]. It remains to show that $\lambda_{\mathcal{F}}$ is also an $F$-isomorphism. Clearly, $\lambda_{\mathcal{F}}$ is an $F$-monomorphism, since $\operatorname{Ker}\left(\lambda_{\mathcal{F}}\right)$ is contained in $\operatorname{Ker}\left(\lambda_{S}\right) \subseteq H^{*}(B S)$, where every element is nilpotent. Thus it remains to show that $\lambda_{\mathcal{F}}$ is an $F$-epimorphism.

Fix some

$$
x=\left(x_{E}\right)_{E \in \mathcal{E}(S)} \in H_{\mathcal{E}}^{*}(\mathcal{F})=\varlimsup_{\lim _{\mathcal{F} e}} H^{*}(-) \subseteq \prod_{E \in \mathcal{E}(S)} H^{*}(B E) .
$$

We must show that there exists some $N>0$ such that $x^{N} \in \operatorname{Im}\left(\lambda_{\mathcal{F}}\right)$. Since

$$
H^{*}(B P) \stackrel{\lambda_{P}}{\longrightarrow} H_{\mathcal{E}}^{*}(P)
$$

is an $F$-isomorphism for each $P \leq S$ by $[\mathrm{Qu}, 6.2]$ again, there exist $k>0$ and $y_{P} \in H^{*}(B P)$ such that for all $E \leq P \leq S$ and all $\varphi \in \operatorname{Hom}_{P}(E, P), \varphi^{*}\left(y_{P}\right)=x_{E}^{p^{k}}$. Since $\mathcal{F}$ has finitely many objects, we may choose $k$ sufficiently large so that this holds for every $P \leq S$. Then by definition of the inverse limit, for each $\psi \in$ $\operatorname{Hom}_{\mathcal{F}}(P, Q), \psi^{*}\left(y_{Q}\right)$ and $y_{P}$ restrict to the same element $x_{E}^{p^{k}}$ for each elementary abelian subgroup $E \leq P$. Since $\lambda_{P}$ is an $F$-monomorphism,

$$
\left(\psi^{*}\left(y_{Q}\right)-y_{P}\right)^{p^{m}}=\psi^{*}\left(y_{Q}\right)^{p^{m}}-y_{P}^{p^{m}}=0
$$

for $m$ sufficiently large. Thus, since there are only finitely many morphisms in $\mathcal{F}$, we can choose $m$ large enough so that $\left(\left(y_{P}\right)_{P \leq S}\right)^{p^{m}}$ is an element of the inverse limit $H^{*}(\mathcal{F})$; and hence $x^{p^{m+k}} \in \operatorname{Im}\left(\lambda_{\mathcal{F}}\right)$.

We are now ready to prove

Proposition 5.2. For any fusion system $\mathcal{F}$ over a p-group $S$, the ring $H^{*}(\mathcal{F})$ is noetherian, and $H^{*}(B S)$ is finitely generated as an $H^{*}(\mathcal{F})$-module.

Proof. We will need to refer to the following well known facts, for any triple $k \subseteq$ $B \subseteq A$ of commutative rings where $k$ is noetherian:

(1) For any $x \in A, B[x]$ is finitely generated as a $B$-module if and only if $x$ is integral over $B$; i.e., if and only if it satisfies a monic polynomial with coefficients in $B$. If $A$ is finitely generated as a $B$-algebra and every element of $A$ is integral over $B$, then $A$ is finitely generated as a $B$-module.

(2) If $A$ is finitely generated as a $k$-algebra and as a $B$-module, then $B$ is finitely generated as a $k$-algebra.

(3) If $B=A^{G}$ for some finite group $G$ of algebra automorphisms, and $A$ is finitely generated as a $k$-algebra, then $A$ is finitely generated as a $B$-module and $B$ is finitely generated as a $k$-algebra. 
Point (1) is shown, for example, in [AM, Proposition 5.1]. Point (2) is a theorem of Artin and Tate [AT], and follows from (1) upon noting that every element of $A$ is integral over the $k$-subalgebra $B^{\prime} \subseteq B$ generated by some finite set of coefficients of monic polynomials satisfied by a set of $k$-algebra generators for $A$ [AM, Proposition 7.8]. Point (3) (the Hilbert-Noether theorem) follows from (1) and (2), since each $a \in A$ satisfies the monic polynomial $\prod_{g \in G}(X-g(a))$.

Let $V$ be an elementary abelian $p$-group whose rank is equal to $\operatorname{rk}_{p}(S)$. Define

$$
\tau: H^{*}(B V)^{G L(V)} \longrightarrow \prod_{E \in \mathcal{E}(S)} H^{*}(B E)
$$

by letting the projection to the coordinate indexed by $E$ be the unique homomorphism induced by any monomorphism $E \longrightarrow V$. Notice that $\tau$ does not depend on the choice of these monomorphisms, since we are restricting to the $G L(V)$ invariants. Then $\tau$ is an injection ( $V \cong E$ for some $E \in \mathcal{E}(S)$ by assumption), and $\operatorname{Im}(\tau) \subseteq H_{\mathcal{E}}^{*}(\mathcal{F})$. Since $H^{\mathrm{ev}}(B V)$ is finitely generated as an $\mathbb{F}_{p}$-algebra, it is finitely generated as an $H^{\mathrm{ev}}(B V)^{G L(V)}$-module, and $H^{\mathrm{ev}}(B V)^{G L(V)}$ is noetherian, by (3). Since $\mathcal{E}(S)$ is finite and $\operatorname{rk}(V) \geq \operatorname{rk}(E)$ for all $E \in \mathcal{E}(S)$, this shows that $\prod_{E \in \mathcal{E}(S)} H^{*}(B E)$ is finitely generated as a module over $H^{\mathrm{ev}}(B V)^{G L(V)}$ via $\tau$.

Consider the following diagram:

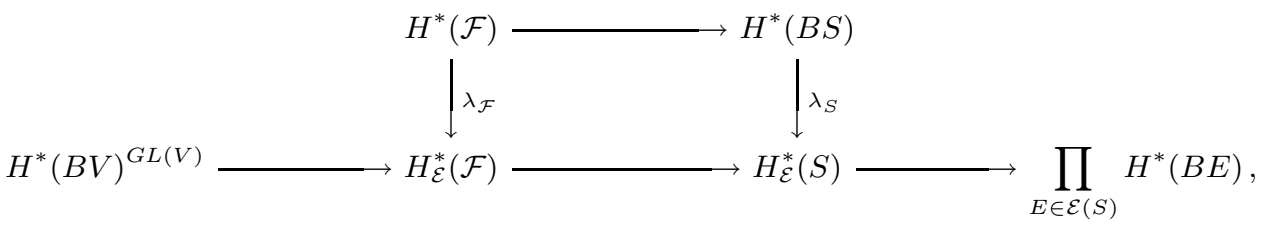

where the horizontal maps are all inclusions, and $\lambda_{\mathcal{F}}$ and $\lambda_{S}$ are $F$-isomorphisms by Proposition 5.1. We have just seen that every element of $H_{\mathcal{E}}^{\mathrm{ev}}(S)$ is integral over $H^{\mathrm{ev}}(B V)^{G L(V)}$ and hence over $H_{\mathcal{E}}^{\mathrm{ev}}(\mathcal{F})$. Thus every element of $H^{\mathrm{ev}}(B S)$ is integral

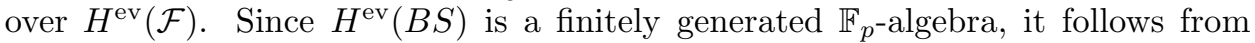
(1) that $H^{\mathrm{ev}}(B S)$ and hence $H^{*}(B S)$ are finitely generated as $H^{\mathrm{ev}}(\mathcal{F})$-modules, and from $(2)$ that $H^{\mathrm{ev}}(\mathcal{F})$ is finitely generated as an $\mathbb{F}_{p}$-algebra. Hence $H^{*}(B S)$ is finitely generated as an $H^{*}(\mathcal{F})$-module, and $H^{*}(\mathcal{F})$ is noetherian since it is a submodule of a finitely generated module over the noetherian $\operatorname{ring} H^{\mathrm{ev}}(\mathcal{F})$.

We next show that $R_{\mathcal{L}}$ is an $F$-isomorphism.

Lemma 5.3. For any $p$-local finite group $(S, \mathcal{F}, \mathcal{L}), H^{*}(|\mathcal{L}|)$ is the limit of a spectral sequence of $H^{*}(|\mathcal{L}|)$-modules, where each column in the $E_{1}$-term is a finite sum of copies of $H^{*}(B P)$ for subgroups $P \leq S$, and where the $E_{2}$-term has only a finite number of nonzero columns. Also, the induced homomorphism

$$
R_{\mathcal{L}}: H^{*}(|\mathcal{L}|) \longrightarrow H^{*}(\mathcal{F})
$$

is an $F$-isomorphism, and $H^{*}(\mathcal{F})$ is finitely generated as an $H^{*}(|\mathcal{L}|)$-module via $R_{\mathcal{L}}$.

Proof. By Proposition [2.2, $|\mathcal{L}| \simeq$ hocolim $(\widetilde{B})$, for some functor $\widetilde{B}: \mathcal{O}^{c}(\mathcal{F}) \longrightarrow$ Top which sends each $P$ to a space with the homotopy type of $B P$. Hence, in the spectral sequence for the cohomology of the homotopy colimit, each column in the $E_{1}$-term is a sum of rings $H^{*}(B P)$ for subgroups $P \leq S$. Also, $E_{2}^{p *} \cong \underset{\mathcal{O}^{c}(\mathcal{F})}{\lim ^{p}}\left(H^{*}(-)\right)$, and hence there are only finitely many nonzero columns by Corollary 3.4 
The cohomology spectral sequence for the homotopy colimit is the same as the cohomology spectral sequence for the projection map

$$
\underset{\mathcal{O}^{c}(\mathcal{F})}{\operatorname{hocolim}}(\widetilde{B}) \longrightarrow\left|\mathcal{O}^{c}(\mathcal{F})\right|
$$

and hence is multiplicative (cf. [Bd, $\S \mathrm{IV} .6 .5]$ ). Since in our case there are only finitely many nonzero columns in the spectral sequence, and since $E_{2}^{0 *}=H^{*}(\mathcal{F})$, it follows at once that that the map $R_{\mathcal{L}}$ is an $F$-monomorphism.

To see that it is an $F$-epimorphism, we must show that for every element $x \in$ $H^{*}(\mathcal{F})$ there is an integer $n$ such that $x^{n} \in \operatorname{Im}\left(R_{\mathcal{L}}\right)$, i.e., such that $x^{n}$ is a permanent cycle in the spectral sequence. Let $x \in E_{2}^{0, k}=H^{k}(\mathcal{F})$ be an element which is not a permanent cycle, and let $r$ be the smallest integer for which $d_{r}(x) \neq 0$. We can assume that $k$ is even if $p$ is odd (otherwise $x^{2}=0$ ). Then $d_{r}\left(x^{p}\right)=$ $p x^{p-1} d_{r}(x)=0$. Again using the fact that there are finitely many nonzero columns in the spectral sequence, iterating this procedure shows that $x^{p^{k}}$ is a permanent cycle for a sufficiently large $k$.

Since $H^{*}(\mathcal{F})$ is noetherian, it is finitely generated as an algebra. Let $\left\{x_{1}, \ldots, x_{k}\right\}$ be a set of homogeneous algebra generators, and for each $i$ fix $m_{i}$ such that $\left(x_{i}\right)^{m_{i}} \in$ $\operatorname{Im}\left(R_{\mathcal{L}}\right)$. Then the elements $\left(x_{i}\right)^{j}$ for $1 \leq i \leq k$ and $1 \leq j \leq m_{i}$ generate $H^{*}(\mathcal{F})$ as a module over $\operatorname{Im}\left(R_{\mathcal{L}}\right)$.

The following technical lemma will be needed.

Lemma 5.4. Fix a saturated fusion system $\mathcal{F}$ over a $p$-group $S$, and let $\mathcal{H}$ be a set of subgroups of $S$ such that for each $H \in \mathcal{H}$, all subgroups of $H$ and all subgroups $\mathcal{F}$-conjugate to $H$ are also in $\mathcal{H}$. Assume $\Omega_{0}$ is an $S$-set with the property that for all $P, P^{\prime} \leq S$ which are $\mathcal{F}$-conjugate and not in $\mathcal{H},\left|\Omega_{0}{ }^{P}\right|=\left|\Omega_{0}{ }^{P^{\prime}}\right|$. Then there is an $S$-set $\Omega \supseteq \Omega_{0}$ such that $\left|\Omega^{P}\right|=\left|\Omega^{P^{\prime}}\right|$ for each pair of $\mathcal{F}$-conjugate subgroups $P, P^{\prime} \leq S$, and such that $\Omega^{P}=\Omega_{0}{ }^{P}$ for all $P \leq S$ not in $\mathcal{H}$. In particular, for all $P \leq S$ and all $\alpha \in \operatorname{Hom}_{\mathcal{F}}(P, S), \Omega$ as a $P$-set via restriction is isomorphic to $\Omega$ as

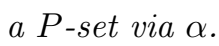

Proof. If $\mathcal{H}=\emptyset$ then the claim holds trivially. Let $\mathcal{H}$ be an arbitrary collection of subgroups satisfying the hypotheses, let $P$ be a maximal subgroup in $\mathcal{H}$, and let $\mathcal{H}^{\prime} \subseteq \mathcal{H}$ be the subset of those subgroups in $\mathcal{H}$ not $\mathcal{F}$-conjugate to $P$. We can assume inductively that the result holds for $\mathcal{H}^{\prime}$. We can also assume that $P$ was chosen to be fully normalized (Definition[1.2), and (after adding orbits of type $S / P$ to $\Omega_{0}$ if necessary) that $\left|\Omega_{0}{ }^{P}\right| \geq\left|\Omega_{0}{ }^{\prime}\right|$ for all $P^{\prime}$ that are $\mathcal{F}$-conjugate to $P$.

Fix $P^{\prime}$ in the $\mathcal{F}$-conjugacy class of $P$. By Proposition $\mathrm{A} .2(\mathrm{~b})$, there is some $\varphi \in \operatorname{Hom}_{\mathcal{F}}\left(N_{S}\left(P^{\prime}\right), N_{S}(P)\right)$ such that $\varphi\left(P^{\prime}\right)=P$. By assumption, for all $P^{\prime} \supsetneqq$ $Q \leq N_{S}\left(P^{\prime}\right)$ we have $\left|\Omega_{0}{ }^{Q}\right|=\left|\Omega_{0} \varphi(Q)\right|$. Hence the sets of elements in nonfree orbits for the actions of $N_{S}\left(P^{\prime}\right) / P^{\prime}$ on $\Omega_{0}{ }^{\prime}$ and (via $\varphi$ ) on $\Omega_{0}{ }^{P}$ have the same order, and SO

$$
\left|\Omega_{0}^{P^{\prime}}\right| \equiv\left|\Omega_{0}^{P}\right| \quad\left(\bmod \left|N_{S}\left(P^{\prime}\right) / P^{\prime}\right|\right)
$$

Set

Now define

$$
n_{P^{\prime}}=\frac{\left|\Omega_{0} P\right|-\left|\Omega_{0}^{P^{\prime}}\right|}{\left|N_{S}\left(P^{\prime}\right) / P^{\prime}\right|} \in \mathbb{N} .
$$

$$
\Omega_{1}=\Omega_{0} \amalg\left(\coprod_{P^{\prime}} n_{P^{\prime}} \cdot\left(S / P^{\prime}\right)\right) \text {, }
$$


where the union is taken over all subgroups $P^{\prime}$ in the $\mathcal{F}$-conjugacy class of $P$, $n_{P^{\prime}} \cdot\left(S / P^{\prime}\right)$ means the disjoint union of $n_{P^{\prime}}$ copies of the set $S / P^{\prime}$, and $n_{P}=0$. Then $\Omega_{1}{ }^{Q}=\Omega_{0}{ }^{Q}$ for $Q \notin \mathcal{H}$, and $\left|\Omega_{1} P^{\prime}\right|=\left|\Omega_{1}{ }^{P}\right|$ for $P^{\prime} \mathcal{F}$-conjugate to $P$. Thus $\left|\Omega_{1} P^{\prime}\right|=\left|\Omega_{1}{ }^{P}\right|$ for all $\mathcal{F}$-conjugate subgroups $P, P^{\prime} \leq S$ which are not in $\mathcal{H}^{\prime}$.

The construction of $\Omega$ now follows by the induction hypothesis, applied to $\Omega_{1}$ and $\mathcal{H}^{\prime}$. The last statement follows since, in general, two finite $P$-sets $\Omega^{\prime}, \Omega^{\prime \prime}$ are $P$-isomorphic if and only if $\left|\left(\Omega^{\prime}\right)^{Q}\right|=\left|\left(\Omega^{\prime \prime}\right)^{Q}\right|$ for all $Q \leq P$.

We need to work with $(S, S)$-bisets: sets having left and right actions of $S$ which commute with each other. We first establish some notation. If $P \leq S$ and $\varphi \in \operatorname{Hom}(P, S)$, then $S \times{ }_{(P, \varphi)} S$ denotes the biset

$$
S \times{ }_{(P, \varphi)} S=(S \times S) / \sim, \quad \text { where } \quad(x, g y) \sim(x \varphi(g), y) \quad \text { for } x, y \in S, g \in P .
$$

Then $S \times{ }_{(P, \varphi)} S$ is free as a left $S$-set (and also as a right $S$-set if $\varphi$ is injective), and every $(S, S)$-biset with free left action is a disjoint union of bisets of this form. For each finite $(S, S)$-biset $B$ whose left $S$-action is free, define an endomorphism $[B]$ of $H^{*}(B S)$ as follows. For each $P \leq S$ and each $\varphi \in \operatorname{Hom}(P, S)$, set

$$
\left[S \times_{(P, \varphi)} S\right]=\left(H^{*}(B S) \stackrel{\varphi^{*}}{\longrightarrow} H^{*}(B P) \stackrel{\operatorname{trf}_{P}}{\longrightarrow} H^{*}(B S)\right),
$$

where $\operatorname{trf}_{P}$ denotes the transfer map. Finally, if $B=\coprod_{i=1}^{k} B_{i}$ is a disjoint union of bisets $B_{i}$ of this form, then $[B]=\sum_{i=1}^{k}\left[B_{i}\right]$.

If $B$ is an $(S, S)$-biset, then for $P \leq S$ and $\varphi \in \operatorname{Inj}(P, S)$, we let $\left.B\right|_{(P, S)}$ denote the restriction of $B$ to a $(P, S)$-biset, and let $\left.B\right|_{(\varphi, S)}$ denote the $(P, S)$-biset where the left $P$-action is induced by $\varphi$.

The following proposition was motivated by recent, unpublished work of Markus Linckelmann and Peter Webb [LW]. They were the ones who formulated conditions (a), (b), and (c) below, and recognized the importance of finding a biset with these properties.

Proposition 5.5. For any saturated fusion system $\mathcal{F}$ over a p-group $S$, there is an $(S, S)$-biset $\Omega$ with the following properties:

(a) Each indecomposable component of $\Omega$ is of the form $S \times_{(P, \varphi)} S$ for some $P \leq S$ and some $\varphi \in \operatorname{Hom}_{\mathcal{F}}(P, S)$.

(b) For each $P \leq S$ and each $\varphi \in \operatorname{Hom}_{\mathcal{F}}(P, S),\left.\Omega\right|_{(P, S)}$ and $\left.\Omega\right|_{(\varphi, S)}$ are isomorphic as $(P, S)$-bisets.

(c) $|\Omega| /|S| \equiv 1(\bmod p)$.

Furthermore, for any biset $\Omega$ which satisfies these properties, $[\Omega]$ is an idempotent in $\operatorname{End}\left(H^{*}\left(B S ; \mathbb{F}_{p}\right)\right)$, is $H^{*}(\mathcal{F})$-linear and a homomorphism of modules over the Steenrod algebra; and

$$
\operatorname{Im}\left[H^{*}\left(B S ; \mathbb{F}_{p}\right) \stackrel{[\Omega]}{\longrightarrow} H^{*}\left(B S ; \mathbb{F}_{p}\right)\right]=H^{*}(\mathcal{F}) .
$$

Note in particular, when $\mathcal{F}$ is the fusion system of a finite group $G$, that $\Omega=G$ (considered as an $(S, S)$-biset) satisfies conditions (a) and (b), and that one can choose $k \geq 1$ such that $\Omega=\coprod^{k} G$ satisfies (c) as well.

Proof. We first assume the existence of $\Omega$, and prove the statements about $[\Omega]$. For any $r \in H^{*}(\mathcal{F})$ and any $\varphi \in \operatorname{Hom}_{\mathcal{F}}(P, S)$ we have $\varphi^{*}(r)=\operatorname{Res}_{P}(r)$ (by definition 
of $H^{*}(\mathcal{F})$ as an inverse limit), and in particular

$$
\left[S \times_{(P, \varphi)} S\right](r)=\operatorname{trf}_{P}\left(\varphi^{*}(r)\right)=\operatorname{trf}_{P} \circ \operatorname{Res}_{P}(r)=[S: P] \cdot r=\frac{\left|S \times_{(P, \varphi)} S\right|}{|S|} \cdot r .
$$

Since $\Omega$ is a disjoint union of such bisets by (a), this shows that $[\Omega](r)=(|\Omega| /|S|) \cdot r$. Hence $[\Omega]$ is the identity on $H^{*}(\mathcal{F})$ by $(\mathrm{c})$. Furthermore, for all $r \in H^{*}(\mathcal{F})$ and all $x \in H^{*}(B S)$,

$$
\left[S \times{ }_{(P, \varphi)} S\right](r x)=\operatorname{trf}_{P}\left(\operatorname{Res}_{P}(r) \cdot \varphi^{*}(x)\right)=r \cdot \operatorname{trf}_{P}\left(\varphi^{*}(x)\right)=r \cdot\left[S \times_{(P, \varphi)} S\right](x),
$$

where the second equality holds by Frobenius reciprocity [Bw, Chapter V, 3.8]. Thus $[\Omega]$ is $H^{*}(\mathcal{F})$-linear. Also, $\operatorname{Im}([\Omega]) \subseteq H^{*}(\mathcal{F})$ by (b), and this shows that $[\Omega]$ is an $H^{*}(\mathcal{F})$-linear splitting of the inclusion $H^{*}(\mathcal{F}) \subseteq H^{*}(B S)$. Finally, $[\Omega]$ is a morphism of modules over the Steenrod algebra, since the $\varphi^{*}$ and the transfer homomorphisms are all such morphisms.

It remains to prove the existence of a biset $\Omega$ satisfying the above conditions. For any $(S, S)$-biset $B$, we also regard $B$ as a set with left $(S \times S)$-action by setting $(g, h) \cdot x=h x g^{-1}$ for $g, h \in S$ and $x \in B$. For each $P \leq S$ and each $\alpha \in \operatorname{Inj}(P, S)$, set

$$
\Delta_{P}^{\alpha}=\{(x, \alpha(x)) \mid x \in P\} .
$$

Then the biset $S \times{ }_{(P, \alpha)} S$ corresponds to the $(S \times S)$-set $(S \times S) / \Delta_{P}^{\alpha}$.

Since $S$ is fully normalized in $\mathcal{F}$, $\operatorname{Out}_{\mathcal{F}}(S)$ has order prime to $p$ by condition (I) in Definition 1.2. So we can choose $k>0$ such that $k \cdot\left|\operatorname{Out}_{\mathcal{F}}(S)\right| \equiv 1(\bmod p)$, and set

$$
\Omega_{0}=k \cdot \coprod_{\alpha \in \operatorname{Out}_{\mathcal{F}}(S)}(S \times S) / \Delta_{S}^{\alpha} .
$$

(Note that if $\alpha, \alpha^{\prime} \in \operatorname{Aut}_{\mathcal{F}}(S)$ are conjugate modulo inner automorphisms, then $\Delta_{S}^{\alpha}$ and $\Delta_{S}^{\alpha^{\prime}}$ are conjugate in $S \times S$, and hence their orbits are isomorphic.) Then $\left|\Omega_{0}\right|=k \cdot\left|\operatorname{Out}_{\mathcal{F}}(S)\right| \cdot|S|$, and $\left|\Omega_{0}\right| /|S| \equiv 1(\bmod p)$.

Let $\mathcal{H}$ be the family of subgroups of $S \times S$

$$
\mathcal{H}=\left\{\Delta_{P}^{\alpha} \mid P \supsetneqq S, \alpha \in \operatorname{Hom}_{\mathcal{F}}(P, S)\right\} .
$$

We must find an $(S \times S)$-set $\Omega \supseteq \Omega_{0}$ with the properties

$\left(\mathrm{a}^{\prime}\right)$ each isotropy subgroup of $\Omega \backslash \Omega_{0}$ lies in $\mathcal{H}$, and

(b') for all $P \leq S$ and all $\alpha \in \operatorname{Hom}_{\mathcal{F}}(P, S), \Omega$ as a $(P \times S)$-set via restriction is isomorphic to $\Omega$ as a $(P \times S)$-set via $(\alpha, \mathrm{Id})$.

Then $\Omega$, when regarded as an $(S, S)$-biset, satisfies conditions (a) and (b) above. Also,

$$
|\Omega| \equiv\left|\Omega_{0}\right| \equiv|S| \quad(\bmod p|S|),
$$

since each orbit $(S \times S) / \Delta_{P}^{\alpha}$ for $P \supsetneqq S$ has order a multiple of $p|S|$, and this proves condition (c).

Now, $\mathcal{F} \times \mathcal{F}$ is a saturated fusion system over $S \times S$ by Lemma 1.5, the collection $\mathcal{H}$ is closed under subgroups and $(\mathcal{F} \times \mathcal{F})$-conjugacy, and all proper subgroups of isotropy subgroups of $\Omega_{0}$ are in $\mathcal{H}$. Hence $\Omega_{0}$ has the feature that for any subgroup $Q \leq S \times S$ which is neither of the form $\Delta_{S}^{\alpha}$ nor in $\mathcal{H}, \Omega_{0}^{Q}=\emptyset$. If $Q=\Delta_{S}^{\alpha}$ and $Q^{\prime}$ is $(\mathcal{F} \times \mathcal{F})$-conjugate to $Q$, then $\left|\Omega_{0}^{Q}\right|=\left|\Omega_{0}^{Q^{\prime}}\right|$ by construction. Hence by Lemma 5.4 
there exists an $(S \times S)$-set $\Omega \supseteq \Omega_{0}$ such that $\left|\Omega^{Q}\right|=\left|\Omega^{Q^{\prime}}\right|$ for any pair of $(\mathcal{F} \times \mathcal{F})$ conjugate subgroups $Q, Q^{\prime} \leq \bar{S} \times S$, and such that $\Omega^{R}=\Omega_{0}^{R}$ for all $R \leq S \times S$ not in $\mathcal{H}$. Conditions $\left(\mathrm{a}^{\prime}\right)$ and $\left(\mathrm{b}^{\prime}\right)$ follow at once.

If $\mathcal{F}$ is a saturated fusion system over a $p$-group $S$, and $\Omega$ is an $(S, S)$-biset which satisfies the conditions of Proposition 5.5, then $\Omega$ induces a stable map from the suspension spectrum $\Sigma^{\infty} B S$ to itself, which is constructed similarly to the endomorphism of $H^{*}(B S)$, but using the stable transfer maps in place of the cohomological transfer. It is not hard to show, using conditions (a)-(c) of the proposition, that the infinite mapping telescope for this stable map is a spectrum $\mathbb{B}_{\mathcal{F}}$ independent of the choice of $\Omega$, and such that $H^{*}\left(\mathbb{B}_{\mathcal{F}}\right) \cong H^{*}(\mathcal{F})$. Furthermore, if $\mathcal{L}$ is any centric linking system associated to $\mathcal{F}$, then $\mathbb{B}_{\mathcal{F}} \simeq \Sigma^{\infty}\left(|\mathcal{L}|_{p}^{\wedge}\right)$. We can thus associate a unique "classifying spectrum" to each saturated fusion system $\mathcal{F}$, whether or not we can associate a classifying space to $\mathcal{F}$. The idea for constructing a spectrum in this way associated to $\mathcal{F}$ is due to Linckelmann and Webb.

For the purposes of the next lemma and later inductive arguments, for any $p$ local finite group $(S, \mathcal{F}, \mathcal{L})$, we say that $H^{*}(|\mathcal{L}|)$ is "computed by stable elements" if the natural map

$$
R_{\mathcal{L}}: H^{*}(|\mathcal{L}|) \longrightarrow H^{*}(\mathcal{F})
$$

is an isomorphism. The goal of course is to show that this always holds.

Lemma 5.6. Fix a p-local finite group $(S, \mathcal{F}, \mathcal{L})$ for which there is a central subgroup $V \leq S$ of order $p$ (i.e., $\left.\mathcal{F}=C_{\mathcal{F}}(V)\right)$. Let $\mathcal{F} / V$ be the induced fusion system over $S / V$. Let $\mathcal{L} / V$ be the category whose objects are the subgroups $P / V \leq S / V$ such that $P$ is $\mathcal{F}$-centric, and where

$$
\operatorname{Mor}_{\mathcal{L} / V}(P / V, Q / V)=\operatorname{Mor}_{\mathcal{L}}(P, Q) / \delta_{P}(V) .
$$

Let $\mathcal{L}_{0} \subseteq \mathcal{L}$ and $(\mathcal{L} / V)^{c} \subseteq \mathcal{L} / V$ be the full subcategories whose objects are those $P \leq S$ and $P / V \leq S / V$, respectively, such that $P / V$ is $(\mathcal{F} / V)$-centric. Then the following hold:

(a) $\mathcal{F} / V$ is a saturated fusion system, and $(\mathcal{L} / V)^{c}$ is a centric linking system associated to $\mathcal{F} / V$.

(b) $B V \longrightarrow\left|\mathcal{L}_{0}\right|_{p}^{\wedge} \longrightarrow\left|(\mathcal{L} / V)^{c}\right|_{p}^{\wedge}$ is a fibration sequence.

(c) The inclusion $\left|\mathcal{L}_{0}\right|_{p}^{\wedge} \subseteq|\mathcal{L}|_{p}^{\wedge}$ is a homotopy equivalence.

(d) If $H^{*}\left(\left|(\mathcal{L} / V)^{c}\right|\right)$ is computed by stable elements, then $H^{*}(|\mathcal{L}|)$ is computed by stable elements.

Proof. Since $V$ is central in $\mathcal{F}$, all $\mathcal{F}$-centric subgroups of $S$ contain $V$, and the restriction to $V$ of any morphism in $\mathcal{F}^{c}$ is the identity. Hence by condition (C) in Definition 1.7, for any $P, Q$ in $\mathcal{L}$, the left and right actions of $V$ on $\operatorname{Mor}_{\mathcal{L}}(P, Q)$ (via composition with $\delta_{Q}(V)$ and $\delta_{P}(V)$, respectively) are the same.

(a) For each $P \leq S$ which contains $V$, set

$$
\Gamma_{P}=\operatorname{Ker}\left[\operatorname{Aut}_{\mathcal{F}}(P) \longrightarrow \operatorname{Aut}_{\mathcal{F} / V}(P / V)\right]
$$

and

$$
N_{S}^{0}(P)=\left\{g \in N_{S}(P) \mid c_{g} \in \Gamma_{P}\right\} .
$$


In particular, $N_{S}^{0}(P) / V=C_{S / V}(P / V)$. We first claim that

$$
\text { P fully normalized in } \begin{aligned}
\mathcal{F} & \Longrightarrow P / V \text { fully centralized in } \mathcal{F} / V \\
& \Longrightarrow \Gamma_{P} \leq \operatorname{Aut}_{S}(P) .
\end{aligned}
$$

By definition, any subgroup of $S$ is $\mathcal{F}$-conjugate to a fully normalized subgroup, and any subgroup of $S / V$ is $\mathcal{F} / V$-conjugate to a fully centralized subgroup. Hence it will suffice to prove, for any pair of $\mathcal{F}$-conjugate subgroups $P, Q \leq S$ such that $P / V$ is fully centralized in $\mathcal{F} / V$ and $Q$ is fully normalized in $\mathcal{F}$, that $Q / V$ is fully centralized in $\mathcal{F} / V$ and $\Gamma_{P} \leq \operatorname{Aut}_{S}(P)$. Since $\Gamma_{Q}$ is a normal $p$-subgroup of $\operatorname{Aut}_{\mathcal{F}}(Q)$, and $\operatorname{Aut}_{S}(Q)$ is a Sylow $p$-subgroup by condition (I) in Definition 1.2, we have $\Gamma_{Q} \leq \operatorname{Aut}_{S}(Q)$. It follows that any $\varphi \in \operatorname{Iso}_{\mathcal{F}}(P, Q)$ extends to $\bar{\varphi}$ in $\operatorname{Hom}_{\mathcal{F}}\left(N_{S}^{0}(P), N_{S}^{0}(Q)\right.$ ) by condition (II) (and since $Q$ is fully centralized in $\mathcal{F}$ by $(\mathrm{I}))$; and this factors through a homomorphism

$$
\bar{\varphi} / V \in \operatorname{Hom}_{\mathcal{F} / V}\left(C_{S / V}(P / V), C_{S / V}(Q / V)\right) .
$$

Since $P / V$ is fully centralized in $\mathcal{F} / V, \bar{\varphi} / V$ must be an isomorphism and hence $Q / V$ is also fully centralized. Then $\bar{\varphi}$ is also an isomorphism, so $\Gamma_{P} \leq \operatorname{Aut}_{S}(P)$, and this finishes the proof of (1).

We next show that $\mathcal{F} / V$ is saturated. Fix $\varphi \in \operatorname{Hom}_{\mathcal{F} / V}(P / V, S / V)$ such that $\operatorname{Im}(\varphi)$ is fully centralized in $\mathcal{F} / V$, choose a lifting $\widetilde{\varphi} \in \operatorname{Hom}_{\mathcal{F}}(P, S)$ of $\varphi$, and set $Q=\operatorname{Im}(\widetilde{\varphi})$. Consider the subgroups

$$
N_{\varphi}=\left\{g \in N_{S / V}(P / V) \mid \varphi c_{g} \varphi^{-1} \in \operatorname{Aut}_{S / V}(Q / V)\right\}
$$

and

$$
N_{\widetilde{\varphi}}=\left\{g \in N_{S}(P) \mid \widetilde{\varphi} c_{g} \widetilde{\varphi}^{-1} \in \operatorname{Aut}_{S}(Q)\right\} .
$$

Then $N_{\varphi} \leq N_{\tilde{\varphi}} / V$ by $(1)\left(\Gamma_{Q} \leq \operatorname{Aut}_{S}(Q)\right.$ since $Q / V$ is fully centralized in $\left.\mathcal{F} / V\right)$, so $\varphi$ extends to some $\bar{\varphi} \in \operatorname{Hom}_{\mathcal{F} / V}\left(N_{\varphi}, S / V\right)$ by condition (II) applied to the saturated fusion system $\mathcal{F}$, and this proves condition (II) for $\mathcal{F} / V$. Finally, for $P \leq S$ containing $V, N_{S / V}(P / V)=N_{S}(P) / V$, so $P$ is fully normalized in $\mathcal{F}$ if and only if $P / V$ is fully normalized in $\mathcal{F} / V$. Condition (I) for $\mathcal{F} / V$ now follows from condition (I) for $\mathcal{F}$, since if $P / V \leq S / V$ is fully normalized in $\mathcal{F} / V$, then $P$ is fully normalized in $\mathcal{F}$, so $P / V$ is fully centralized in $\mathcal{F} / V$ by $(1)$, and $\operatorname{Aut}_{S / V}(P / V) \in$ $\operatorname{Syl}_{p}\left(\operatorname{Aut}_{\mathcal{F} / V}(P / V)\right)$ since $\operatorname{Aut}_{S}(P) \in \operatorname{Syl}_{p}\left(\operatorname{Aut}_{\mathcal{F}}(P)\right.$ ) (by condition (I) again for $\mathcal{F})$.

It remains to show that $(\mathcal{L} / V)^{c}$ is a centric linking system associated to $\mathcal{F} / V$. The distinguished monomorphisms

$$
\delta_{P / V}: P / V \longrightarrow \operatorname{Aut}_{\mathcal{L} / V}(P / V)=\operatorname{Aut}_{\mathcal{L}}(P) / V
$$

are induced by the distinguished monomorphisms $\delta_{P}$ for $\mathcal{L}$. Conditions (B) and (C) in Definition 1.7 follow directly for $(\mathcal{L} / V)^{c}$ from the corresponding conditions for $\mathcal{L}$. It remains to prove condition $(\mathrm{A})$.

By construction, the functor $\pi / V:(\mathcal{L} / V)^{c} \longrightarrow(\mathcal{F} / V)^{c}$ is the identity on objects and surjective on morphisms. The only difficulty is to show that each $(\pi / V)_{P / V, Q / V}$ is the orbit map for the free action of $Z(P / V)$ on $\operatorname{Mor}_{\mathcal{L} / V}(P / V, Q / V)$ via $\delta_{P / V}$. Fix $P$ and $Q$ such that $P / V$ and $Q / V$ are $(\mathcal{F} / V)$-centric, and consider the following 
commutative square:

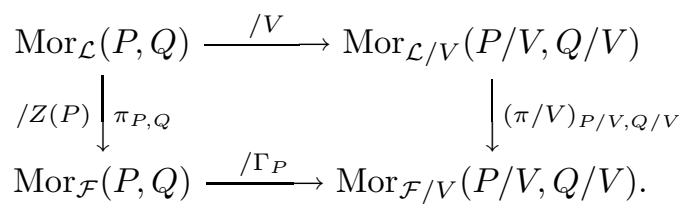

Here, a label " $/ H$ " means that the map is an orbit map for an action of $H$ on the source set. Since $P / V$ is $\mathcal{F} / V$-centric (and hence fully centralized in $\mathcal{F} / V$ ), $\Gamma_{P}$ is the group of all $c_{g} \in \operatorname{Aut}(P)$ such that $g V \in Z(P / V)$ by (1), and hence $(\pi / V)_{P / V, Q / V}$ is the orbit map for the (free) action of $\delta_{P / V}(Z(P / V))$.

(b) Using Lemma 1.10(a), one checks that each undercategory for the projection of $\mathcal{L}_{0}$ onto $(\mathcal{L} / V)^{c}$ contains a category equivalent to $\mathcal{B}(V)$ as a deformation retract. The map $\left|\mathcal{L}_{0}\right| \longrightarrow\left|(\mathcal{L} / V)^{c}\right|$ thus has homotopy fiber $B V$ by Quillen's Theorem B. This also follows more directly using the lemma in Qu2, p.90], which shows that this map is a quasifibration.

By $\left[\mathrm{BK}\right.$ II.5.1], the fibration sequence $B V \longrightarrow\left|\mathcal{L}_{0}\right| \longrightarrow\left|(\mathcal{L} / V)^{c}\right|$ is still a fibration sequence after $p$-completion.

(c) Fix $P \leq S$ in $\mathcal{L}$ but not in $\mathcal{L}_{0}$. Thus $P$ is $\mathcal{F}$-centric (and hence $P \supseteq V$ ), but $P / V$ is not $(\mathcal{F} / V)$-centric. We can assume, after replacing $P$ by another subgroup in its $\mathcal{F}$-conjugacy class if necessary, that $C_{S / V}(P / V) \neq Z(P / V)$. So there is an element $g V \in S / V \backslash P / V$ such that $[g V, P / V]=1$, or equivalently an element $g \in S \backslash P$ such that $[g, P]=V$. Furthermore, $c_{g} \in \operatorname{Aut}(P)$ cannot be an inner automorphism, because if $c_{g}=c_{x}$ for $x \in P$, then $g x^{-1} \in C_{S}(P) \backslash P=\emptyset$, since $P$ is $\mathcal{F}$-centric. Thus $c_{g}$ is a nontrivial element in $\operatorname{Ker}\left[\operatorname{Out}_{\mathcal{F}}(P) \rightarrow \operatorname{Out}_{\mathcal{F}}(P / V)\right]$, so $\operatorname{Out}_{\mathcal{F}}(P)$ has a nontrivial normal $p$-subgroup.

The subcategory $\mathcal{L}_{0}$ thus contains all $\mathcal{F}$-radical $\mathcal{F}$-centric subgroups of $S$. So by Corollary 3.6, the inclusion $\mathcal{L}_{0} \subseteq \mathcal{L}$ induces a homotopy equivalence $\left|\mathcal{L}_{0}\right|_{p}^{\wedge} \simeq|\mathcal{L}|_{p}^{\wedge}$. (d) Fix an $(S, S)$-biset $\Omega$ which satisfies conditions (a)-(c) in Proposition 5.5 for $\mathcal{F}$. We can assume that $\Omega$ is a union of orbits $S \times{ }_{(P, \alpha)} S$ for subgroups $P \leq S$ which contain $V$ (and $\alpha \in \operatorname{Hom}_{\mathcal{F}}(P, S)$ ), since otherwise we can replace $\Omega$ by the fixed point set of the conjugation action of $V$ and it still satisfies the same conditions. Set $\bar{\Omega}=V \backslash \Omega / V$, regarded as an $(S / V, S / V)$-biset. Conditions (b) and (c) clearly hold. Also, each orbit $S \times_{(P, \alpha)} S$ in $\Omega$ corresponds to an orbit $(S / V) \times_{(P / V, \bar{\alpha})}(S / V)$ in $\bar{\Omega}$, which shows that $\bar{\Omega}$ also satisfies condition (a) and has the same number of orbits as $\Omega$.

Fix a $\mathbb{Z}[S]$-free resolution $M_{*}$ of $\mathbb{Z}$, and a $\mathbb{Z}[S / V]$-free resolution $M_{*}^{\prime}$ of $\mathbb{Z}$. For each $P \leq S$, the spectral sequence $E_{*}(P)$ for the extension

$$
1 \longrightarrow V \longrightarrow P \longrightarrow P / V \longrightarrow 1
$$

is induced by the double complex

$$
\operatorname{Hom}_{\mathbb{Z}[P]}\left(M_{i}^{\prime} \otimes M_{j}, \mathbb{F}_{p}\right) \cong \operatorname{Hom}_{\mathbb{Z}[P / V]}\left(M_{i}^{\prime}, \operatorname{Hom}_{\mathbb{Z}[V]}\left(M_{j}, \mathbb{F}_{p}\right)\right) .
$$

The transfer maps $\operatorname{Hom}_{\mathbb{Z}[P]}(A, B) \longrightarrow \operatorname{Hom}_{\mathbb{Z}[S]}(A, B)$, which send $f$ to $\sum g_{i} f g_{i}^{-1}$ (the sum taken over a set of coset representatives for $P$ in $S$ ) induce a homomorphism $E_{*}(P) \stackrel{\operatorname{trf}}{\longrightarrow} E_{*}(S)$ of spectral sequences, which in the $E_{2}$-term is the usual transfer map $H^{*}(P / V) \longrightarrow H^{*}(S / V)$ in each row, and which in the $E_{\infty}$-term is the map on associated graded modules induced by the transfer map $H^{*}(P) \longrightarrow H^{*}(S)$. 
Thus, for each $P \leq S$ and each $\alpha \in \operatorname{Hom}_{\mathcal{F}}(P, S)$, the biset $S \times_{(P, \alpha)} S$ induces a homomorphism of spectral sequences

$$
E_{*}(S) \stackrel{\alpha^{*}}{\longrightarrow} E_{*}(P) \stackrel{\operatorname{trf}}{\longrightarrow} E_{*}(S) .
$$

Upon summing these over all orbits in the biset $\Omega$, we get a homomorphism

$$
E_{*}(S) \stackrel{\Omega_{*}}{\longrightarrow} E_{*}(S)
$$

which induces the endomorphism $[\bar{\Omega}]$ of $H^{*}(S / V)$ on each row in $E_{2}(S)$, and which converges to $[\Omega]$. In particular, $\Omega_{*}$ is idempotent on $E_{2}(S)$, hence on $E_{r}(S)$ for all $r \geq 2$, and thus splits $E_{*}(S)$ as a sum of two spectral sequences. It follows that $E_{*}(S)$ restricts to a spectral sequence

$$
E_{2}^{i, j}=H^{i}(\mathcal{F} / V) \otimes H^{j}(B V) \Longrightarrow H^{i+j}(\mathcal{F}) .
$$

Furthermore, the spectral sequence for the fibration $B V \longrightarrow\left|\mathcal{L}_{0}\right| \longrightarrow\left|(\mathcal{L} / V)^{c}\right|$ maps to this one, and is an isomorphism on $E_{2}$-terms since

$$
H^{*}\left(\left|(\mathcal{L} / V)^{c}\right|\right) \cong H^{*}(\mathcal{F} / V)
$$

by assumption. This proves that $H^{*}(|\mathcal{L}|) \cong H^{*}\left(\left|\mathcal{L}_{0}\right|\right) \cong H^{*}(\mathcal{F})$, and thus that $H^{*}(|\mathcal{L}|)$ is computed by stable elements.

One more lemma is needed, to handle the $p$-local finite groups which do not contain nontrivial central subgroups. We refer to Definition A.3, or to the discussion before Definition 2.4, for the definition of the centralizer fusion system $C_{\mathcal{F}}(Q)$, when $Q$ is a fully centralized subgroup in a fusion system $\mathcal{F}$. By Proposition A.6 $C_{\mathcal{F}}(Q)$ is saturated if $\mathcal{F}$ is saturated.

Lemma 5.7. Fix a p-local finite group $(S, \mathcal{F}, \mathcal{L})$, and let $H^{*}(\mathcal{F}) \subseteq H^{*}(B S)$ be the subring of stable elements for $\mathcal{F}$. Let $E \leq S$ be an elementary abelian subgroup which is fully centralized in $\mathcal{F}$, and let $j_{E}: H^{*}(\mathcal{F}) \longrightarrow H^{*}(B E)$ be the map induced by inclusion. Then there is an isomorphism

$$
T_{E}\left(H^{*}(\mathcal{F}) ; j_{E}\right) \stackrel{\cong}{\longrightarrow} H^{*}\left(C_{\mathcal{F}}(E)\right) \stackrel{\text { def }}{=} \lim _{\mathcal{O}\left(\overleftarrow{C_{\mathcal{F}}}(E)\right)} H^{*}(-)
$$

which is the restriction of the homomorphism

$$
T_{E}\left(H^{*}(B S) ; j_{E}\right) \longrightarrow H^{*}\left(C_{S}(E)\right)
$$

induced by the natural homomorphism $C_{S}(E) \times E \longrightarrow S$.

Proof. Since the functor $T_{E}$ is exact and commutes with direct limits, it commutes with inverse limits over finite categories. So there is an isomorphism

$$
T_{E}\left(H^{*}(\mathcal{F})\right)=T_{E}\left(\varliminf_{\mathcal{O}(\mathcal{F})} H^{*}(-)\right) \cong \lim _{\mathcal{O}(\mathcal{F})} T_{E}\left(H^{*}(-)\right)
$$

It remains to restrict to those summands which extend $j_{E}$. For each $P \leq S$, let $\mathcal{T}_{P}$ be the set of elements $\rho \in \operatorname{Rep}(E, P)$ which are $\mathcal{F}$-conjugate (as homomorphisms to $S$ ) to the inclusion $E \hookrightarrow S$. Then

$$
T_{E}\left(H^{*}(\mathcal{F}) ; j_{E}\right) \cong \lim _{P \in \mathcal{O}(\mathcal{F})}\left(\bigoplus_{\rho \in \mathcal{T}_{P}} T_{E}\left(H^{*}(B P) ; \rho^{*}\right)\right) \cong \lim _{P \in \mathcal{O}(\mathcal{F})}\left(\bigoplus_{\rho \in \mathcal{T}_{P}} H^{*}\left(B C_{P}(\rho E)\right)\right) .
$$


Let $\mathcal{O}_{E}(\mathcal{F})$ denote the category whose objects are the pairs $(P, \rho)$ for all $P \leq S$ and $\rho \in \mathcal{T}_{P}$, and where $\operatorname{Mor}_{\mathcal{O}_{E}(\mathcal{F})}((P, \rho),(Q, \sigma))$ is the subset of those elements $\alpha \in \operatorname{Rep}_{\mathcal{F}}(P, Q)$ such that $\alpha \circ \rho=\sigma$. Then the above formula takes the form

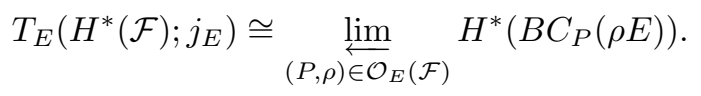

The right hand side can be simplified as follows. Since $E$ is abelian, any injection $\rho: E \longrightarrow P$ takes values in $P \cap C_{S}(\rho E)$. If $\alpha \in \operatorname{Rep}_{\mathcal{F}}(P, Q)$ is such that $\alpha \circ \rho=\sigma$, then for each $x \in C_{S}(\rho E), \alpha(x)$ centralizes $\sigma E$. Furthermore, if we let $P^{\prime}$ denote $P \cap C_{S}(\rho E)$, then $C_{P}(\rho E)=C_{P^{\prime}}(\rho E)$. Hence each object $(P, \rho)$ in $\mathcal{O}_{E}(\mathcal{F})$ may be replaced by $\left(P \cap C_{S}(\rho E), \rho\right)$ with the same morphisms and the same functor without changing the value of the inverse limit. Notice in particular that for each object of the form $\left(P \cap C_{S}(\rho E), \rho\right)$, we have $P \cap C_{S}(\rho E) \leq C_{S}(\rho E)$. Thus we may restrict to the subcategory $\mathcal{O}_{E}^{\prime}(\mathcal{F})$ of those objects $(P, \rho)$ such that $P \leq C_{S}(\rho E)$. Since $E$ is fully centralized, each such object is isomorphic to one where $\rho$ is the inclusion, and the full subcategory of these objects is just $C_{\mathcal{F}}(E)$. So (2) implies (1), and this finishes the proof of the lemma.

We are now ready to show that $H^{*}(|\mathcal{L}|)$ is computed by stable elements, for any $p$-local finite group $(S, \mathcal{F}, \mathcal{L})$.

Theorem 5.8. For any p-local finite group $(S, \mathcal{F}, \mathcal{L})$, the natural homomorphism

$$
R_{\mathcal{L}}: H^{*}\left(|\mathcal{L}|_{p}^{\wedge} ; \mathbb{F}_{p}\right) \stackrel{\cong}{\longrightarrow} H^{*}(\mathcal{F}) \stackrel{\text { def }}{=} \lim _{\mathcal{O}^{c}(\mathcal{F})} H^{*}\left(-; \mathbb{F}_{p}\right)
$$

is an isomorphism, and the ring $H^{*}\left(|\mathcal{L}|_{p}^{\wedge} ; \mathbb{F}_{p}\right)$ is noetherian.

Proof. The second claim follows at once from the first together with Proposition 5.2. It thus remains to prove that $R_{\mathcal{L}}$ is an isomorphism.

Assume inductively that the theorem holds for all "smaller" fusion systems, i.e., for any $p$-local finite group $\left(S^{\prime}, \mathcal{F}^{\prime}, \mathcal{L}^{\prime}\right)$ such that $\left|S^{\prime}\right|<|S|$, or such that $S^{\prime}=S$ and $\mathcal{F}^{\prime}$ is contained in $\mathcal{F}$ as a proper subcategory.

A subgroup $Q \leq Z(S)$ will be called central in $\mathcal{F}$ if $\mathcal{F}=C_{\mathcal{F}}(Q)$, i.e., if each morphism in $\mathcal{F}$ extends to a morphism between subgroups containing $Q$ which is the identity on $Q$.

Case 1: Assume first that $\mathcal{F}$ contains a nontrivial central subgroup. Fix some $V \leq S$ of order $p$ such that $\mathcal{F}=C_{\mathcal{F}}(V)$. Then $\left(S / V, \mathcal{F} / V,(\mathcal{L} / V)^{c}\right)$ is a $p$-local finite group by Lemma [5.6(a), and $H^{*}\left(\left|(\mathcal{L} / V)^{c}\right|\right)$ is computed by stable elements by the induction hypothesis. So $H^{*}(|\mathcal{L}|)$ is computed by stable elements by Lemma 5.6.(d) in this case.

Case 2: Now assume that $\mathcal{F}$ does not contain any nontrivial central subgroup. Set $R=H^{*}(\mathcal{F})$ for short. Thus $R$ is the ring of stable elements for $\mathcal{F}$, regarded as a subring of $H^{*}(B S)$, and we must show that the natural homomorphism $H^{*}(|\mathcal{L}|) \stackrel{R_{\mathcal{L}}}{\longrightarrow} R$ is an isomorphism.

We want to apply [DW1, Theorem 1.2] to the inclusion of algebras $R \subseteq H^{*}(B S)$. The algebra $H^{*}(B S)$ has a "nontrivial center" in the sense of [DW1 $\S 4$ ] since $S$ has nontrivial center. By Proposition 5.2, the ring $R$ is noetherian and $H^{*}(B S)$ is finitely generated as an $R$-module. By Proposition [5.5, there is an $(S, S)$-biset $\Omega$ such that $[\Omega] \in \operatorname{End}\left(H^{*}(B S)\right)$ is an idempotent which defines a left inverse to the 
inclusion $R \subseteq H^{*}(B S)$. Furthermore, this left inverse is a morphism of $R$-modules and a morphism of modules over the Steenrod algebra.

The inclusion $R \subseteq H^{*}(B S)$ thus satisfies the hypotheses of [DW1, Theorem 1.2]. Hence

$$
R \stackrel{\cong}{\lim _{\mathbf{A}(R)}^{0}}\left(\alpha_{R}\right)=\lim _{\overleftarrow{\mathbf{A}(R)}} \alpha_{R}
$$

is an isomorphism, and

$$
{\underset{\lim }{\mathbf{A}(R)}}^{i}\left(\alpha_{R}\right)=0
$$

for all $i>0$. It remains to explain what this means, and to show that this implies that $R \cong H^{*}\left(|\mathcal{L}|_{p}^{\wedge}\right)$.

Let $\mathcal{K}$ be the category of unstable algebras over the mod $p$ Steenrod algebra. For any $K$ in $\mathcal{K}, \mathbf{A}(K)$ denotes the category whose objects are the pairs $(V, f)$, where $V \neq 0$ is an elementary abelian $p$-group and $f \in \operatorname{Mor}_{\mathcal{K}}\left(K, H^{*}(B V)\right)$ makes $H^{*}(B V)$ into a finitely generated $K$-module. A morphism in $\mathbf{A}(K)$ from $(V, f)$ to $\left(V^{\prime}, f^{\prime}\right)$ is a monomorphism $V \stackrel{\varphi}{\longrightarrow} V^{\prime}$ such that $\varphi^{*} f^{\prime}=f$. The functor $\alpha_{K}: \mathbf{A}(K) \longrightarrow \mathcal{K}$ is defined by setting $\alpha_{K}(V, f)=T_{V}(K ; f)$, where $T_{V}$ is Lannes's $T$-functor and $T_{V}(K ; f)$ is the component in $T_{V}(K)$ of $f \in T_{V}^{0}(K) \cong \operatorname{Hom}_{\mathcal{K}}\left(K, H^{*}(B V)\right)$.

Define a functor

$$
\theta: \mathcal{F}^{e} \longrightarrow \mathbf{A}(R)
$$

by setting $\theta(E)=\left(E, i^{*}\right)$, where $R \subseteq H^{*}(B S) \stackrel{i^{*}}{\longrightarrow} H^{*}(B E)$ is induced by the inclusion $E \subseteq S$. This is well defined on morphisms by definition of $R$ as the ring of stable elements in $H^{*}(B S)$. We next show that $\theta$ is an equivalence of categories.

For any space $X$, let $\mathbf{A}(X)$ denote the category whose objects are the pairs $(E, f)$, where $E \neq 1$ is an elementary abelian $p$-group, and $f: B E \longrightarrow X$ makes $H^{*}(B E)$ into a finitely generated $H^{*}(X)$-module. A morphism from $(E, f)$ to $\left(E^{\prime}, f^{\prime}\right)$ is a monomorphism $E \stackrel{\varphi}{\longrightarrow} E^{\prime}$ such that $f^{\prime} \circ B \varphi \simeq f$. Consider the functors

$$
\mathcal{F}^{e} \stackrel{B(-)}{\simeq} \mathbf{A}\left(|\mathcal{L}|_{p}^{\wedge}\right) \stackrel{H^{*}(-)}{\simeq} \mathbf{A}\left(H^{*}(|\mathcal{L}|)\right) \text {. }
$$

The second is an equivalence by [La, Theorem 3.1.1]. Also, $H^{*}(B S)$ is finitely generated as an $H^{*}(|\mathcal{L}|)$-module by Proposition 5.2 and Lemma 5.3 So the first functor is an equivalence by Corollary 4.5 , together with a result of Swan $\underline{\mathrm{Sw}}$ (see also [BLO, Lemma 2.3]) that for $\rho \in \operatorname{Hom}(E, S), H^{*}(B E)$ is a finitely generated $H^{*}(B S)$-module via $B \rho^{*}$ if and only if $\rho$ is injective.

The above composite is thus an equivalence, and sends $E \leq S$ to the homomorphism $H^{*}(|\mathcal{L}|) \longrightarrow H^{*}(E)$ induced by the inclusion of $B E$ in $|\mathcal{L}|$. It is thus equal to the composite

$$
\mathcal{F}^{e} \stackrel{\theta}{\longrightarrow} \mathbf{A}(R) \stackrel{\mathbf{A}\left(R_{\mathcal{L}}\right)}{\longrightarrow} \mathbf{A}\left(H^{*}(|\mathcal{L}|)\right) \text {. }
$$

By Lemma 5.3, $H^{*}(|\mathcal{L}|) \stackrel{R_{\mathcal{L}}}{\longrightarrow} R$ is an $F$-isomorphism, and hence $\mathbf{A}\left(R_{\mathcal{L}}\right)$ is an equivalence of categories by [LS, Corollary 6.5.2]. It follows that $\theta$ is also an equivalence of categories.

By Lemma 5.7, for each $E$ in $\mathcal{F}^{e}, \alpha_{R}(\theta(E))$ is isomorphic to the ring of stable elements $H^{*}\left(C_{\mathcal{F}}(E)\right)$. Since $E$ is never central in $\mathcal{F}$ by assumption, $C_{\mathcal{F}}(E)$ is strictly contained in $\mathcal{F}$, and hence $H^{*}\left(C_{\mathcal{F}}(E)\right) \cong H^{*}\left(\left|C_{\mathcal{L}}(E)\right|\right)$ by the induction hypothesis. It follows that the composite functor $\alpha_{R} \circ \theta$ is naturally isomorphic to the functor which sends $E$ to $H^{*}\left(\left|C_{\mathcal{L}}(E)\right|\right)$. 
By Theorem 2.6, there are a homotopy equivalence

$$
|\mathcal{L}| \simeq \underset{E \in\left(\mathcal{F}^{e}\right)^{o p}}{\stackrel{\text { hocolim }}{\longrightarrow}}\left|\bar{C}_{\mathcal{L}}(E)\right|
$$

and natural isomorphisms

$$
H^{*}\left(\left|\bar{C}_{\mathcal{L}}(E)\right|\right) \cong H^{*}\left(\left|C_{\mathcal{L}}(E)\right|\right) \cong \alpha_{R} \circ \theta(E) .
$$

So there is a spectral sequence

$$
E_{2}^{i *}={\underset{\mathcal{F}}{\lim ^{e}}}^{i}\left(\alpha_{R} \circ \theta\right) \cong{\underset{\mathbf{l i m}}{\mathbf{A}(R)}}^{i}\left(\alpha_{R}\right) \Longrightarrow H^{*}(|\mathcal{L}|) .
$$

Points (1) and (2) now apply to show that this spectral sequence collapses, and that

$$
H^{*}(|\mathcal{L}|) \cong \lim _{\overleftarrow{\mathbf{A}(R)}}\left(\alpha_{R}\right) \cong R
$$

\section{Normalizers, CENTralizers, AND MAPPing SPACES}

We now want to describe more precisely the mapping $\operatorname{spaces} \operatorname{Map}\left(B Q,|\mathcal{L}|_{p}^{\wedge}\right)$, when $(S, \mathcal{F}, \mathcal{L})$ is a $p$-local finite group and $Q$ is a $p$-group. For a finite group $G$, the components of $\operatorname{Map}\left(B Q, B G_{p}^{\wedge}\right)$ are described via centralizers of images of homomorphisms from $Q$ to $G$. By analogy, the components of $\operatorname{Map}\left(B Q,|\mathcal{L}|_{p}^{\wedge}\right)$ will be described via centralizers in the $p$-local finite group $(S, \mathcal{F}, \mathcal{L})$.

Recall that if $\mathcal{F}$ is a saturated fusion system over a $p$-group $S$, and if $Q \leq S$, we say that $Q$ is fully normalized in $\mathcal{F}$ if $\left|N_{S}(Q)\right| \geq\left|N_{S}\left(Q^{\prime}\right)\right|$ for all $Q^{\prime} \mathcal{F}$-conjugate to $Q$. For such $Q, N_{\mathcal{F}}(Q)$ is the fusion system over $N_{S}(Q)$ for which

$$
\begin{aligned}
& \operatorname{Hom}_{N_{\mathcal{F}}(Q)}\left(P, P^{\prime}\right) \\
& \quad=\left\{\varphi \in \operatorname{Hom}_{\mathcal{F}}\left(P, P^{\prime}\right)\left|\exists \psi \in \operatorname{Hom}_{\mathcal{F}}\left(P Q, P^{\prime} Q\right), \psi\right|_{P}=\varphi, \psi(Q)=Q\right\}
\end{aligned}
$$

(see Definition A.3). This is a saturated fusion system by Proposition A.6

Definition 6.1. Fix a $p$-local finite group $(S, \mathcal{F}, \mathcal{L})$, and a $\operatorname{subgroup} Q \leq S$ which is fully normalized in $\mathcal{F}$. Let $N_{\mathcal{L}}(Q)$ be the category whose objects are the $N_{\mathcal{F}}(Q)$ centric subgroups of $N_{S}(Q)$, and where

$$
\operatorname{Mor}_{N_{\mathcal{L}}(Q)}\left(P, P^{\prime}\right)=\left\{\varphi \in \operatorname{Mor}_{\mathcal{L}}\left(P Q, P^{\prime} Q\right) \mid \pi(\varphi)(P) \leq P^{\prime}, \pi(\varphi)(Q)=Q\right\} .
$$

A projection functor $\pi^{N}: N_{\mathcal{L}}(Q) \longrightarrow N_{\mathcal{F}}(Q)$ is defined to be the inclusion on objects, and to send $\varphi \in \operatorname{Mor}_{N_{\mathcal{L}}(Q)}\left(P, P^{\prime}\right)$ to $\left.\pi(\varphi)\right|_{P}$. For each $N_{\mathcal{F}}(Q)$-centric subgroup $P \leq N_{S}(Q)$, the distinguished monomorphism $\delta_{P}^{N}: P \longrightarrow \operatorname{Aut}_{N_{\mathcal{L}}(Q)}(P)$ is defined to be the restriction of the distinguished monomorphism $\delta_{P Q}$ for $\mathcal{L}$.

Note that the following lemma is needed just to know that $N_{\mathcal{L}}(Q)$ is well defined.

Lemma 6.2. Fix a p-local finite group $(S, \mathcal{F}, \mathcal{L})$, and a subgroup $Q \leq S$ which is fully normalized in $\mathcal{F}$. Then for every $P \leq N_{S}(Q)$ which is $N_{\mathcal{F}}(Q)$-centric, $P Q$ is $\mathcal{F}$-centric. Furthermore, $N_{\mathcal{L}}(Q)$ is a centric linking system associated to the saturated fusion system $N_{\mathcal{F}}(Q)$.

Proof. We first prove, for each $N_{\mathcal{F}}(Q)$-centric subgroup $P \leq N_{S}(Q)$, that $P Q$ is $\mathcal{F}$-centric. This means showing, for each $\varphi \in \operatorname{Hom}_{\mathcal{F}}(P Q, S)$, that $C_{S}(\varphi(P Q)) \leq$ $\varphi(P Q)$. Since $Q$ is fully normalized in $\mathcal{F}$, there is by Proposition A.2(b) a morphism

$$
\psi \in \operatorname{Hom}_{\mathcal{F}}\left(N_{S}(\varphi Q), S\right)
$$


such that $\psi(\varphi(Q))=Q$. Thus $\psi \varphi$ is a morphism in $N_{\mathcal{F}}(Q)$. Since $C_{S}(\varphi(P Q)) \leq$ $N_{S}(\varphi Q)$,

$$
\psi\left(C_{S}(\varphi(P Q))\right) \leq C_{S}(\psi \varphi(P Q)) \leq C_{S}(\psi \varphi(P)) \cap N_{S}(Q) \leq \psi \varphi(P),
$$

where the last inequality holds since $P$ is $N_{\mathcal{F}}(Q)$-centric. Thus $C_{S}(\varphi(P Q)) \leq \varphi(P)$, and this finishes the proof that $P Q$ is $\mathcal{F}$-centric.

This shows that Definition 6.1 makes sense. It remains to show that $N_{\mathcal{L}}(Q)$ is a centric linking system associated to $N_{\mathcal{F}}(Q)$. Conditions (B) and (C) in Definition 1.7 follow immediately from the corresponding conditions on $\mathcal{L}$.

To see condition (A), fix $N_{\mathcal{F}}(Q)$-centric subgroups $P, P^{\prime} \leq N_{S}(Q)$. Then $Z(P)$ acts freely on $\operatorname{Mor}_{N_{\mathcal{L}}(Q)}\left(P, P^{\prime}\right)$, since $P Q$ acts freely on $\operatorname{Mor}_{\mathcal{L}}\left(P Q, P^{\prime} Q\right)$ by composition. If $\varphi_{1}, \varphi_{2} \in \operatorname{Mor}_{N_{\mathcal{L}}(Q)}\left(P, P^{\prime}\right)$ are such that $\pi^{N}\left(\varphi_{1}\right)=\pi^{N}\left(\varphi_{2}\right)$, i.e., such that $\left.\pi\left(\varphi_{1}\right)\right|_{P}=\left.\pi\left(\varphi_{2}\right)\right|_{P}$, then since $P$ is $N_{\mathcal{F}}(Q)$-centric, Proposition A.8 applies to show that there is some $g \in Z(P)$ such that $\pi\left(\varphi_{2}\right)=\pi\left(\varphi_{1}\right) \circ c_{g}$. Hence by condition (A) applied to $\mathcal{L}$, there is $h \in Z(P Q) \leq Z(P)$ such that in $\operatorname{Mor}_{\mathcal{L}}\left(P Q, P^{\prime} Q\right)$,

$$
\varphi_{2}=\left(\varphi_{1} \circ \delta_{P Q}(g)\right) \circ \delta_{P Q}(h)=\varphi_{1} \circ \delta_{P}^{N}(g h) .
$$

Thus $\left(\pi^{N}\right)_{P, P^{\prime}}$ is the orbit map for the action of $Z(P)$ on $\operatorname{Mor}_{N_{\mathcal{L}}(Q)}\left(P, P^{\prime}\right)$, and this proves condition (A).

Using Proposition 2.5, for any $p$-local finite group $(S, \mathcal{F}, \mathcal{L})$ and any $Q \leq S$ which is fully centralized in $\mathcal{F}$, we define a functor

$$
\Gamma=\Gamma_{\mathcal{L}, Q}: C_{\mathcal{L}}(Q) \times \mathcal{B}(Q) \longrightarrow \mathcal{L}
$$

by setting $\Gamma\left(P, o_{Q}\right)=P Q$ for each $C_{\mathcal{L}}(Q)$-centric $P \leq C_{S}(Q)$, and

$$
\Gamma_{\left(P, o_{Q}\right),\left(P^{\prime}, o_{Q}\right)}(\varphi, \check{g})=\varphi \circ \widehat{g}=\widehat{g} \circ \varphi \text {. }
$$

The last equality follows from condition $(\mathrm{C})$, since the underlying homomorphism of $\varphi \in \operatorname{Mor}_{\mathcal{L}}\left(P Q, P^{\prime} Q\right)$ is the identity on $Q$. Let

$$
\Gamma_{\mathcal{L}, Q}^{\prime}:\left|C_{\mathcal{L}}(Q)\right|_{p}^{\wedge} \longrightarrow \operatorname{Map}\left(B Q,|\mathcal{L}|_{p}^{\wedge}\right)_{\text {incl }}
$$

be the map adjoint to $|\Gamma|$.

Theorem 6.3. Fix a p-local finite group $(S, \mathcal{F}, \mathcal{L})$, a finite $p$-group $Q$, and a homomorphism $\rho: Q \longrightarrow S$ such that $\rho Q$ is fully centralized in $\mathcal{F}$. Then

$$
\Gamma_{\mathcal{L}, \rho Q}^{\prime}:\left|C_{\mathcal{L}}(\rho Q)\right|_{p}^{\wedge} \simeq \operatorname{Map}\left(B Q,|\mathcal{L}|_{p}^{\wedge}\right)_{B \rho}
$$

is a homotopy equivalence. In particular, $\operatorname{Map}\left(B Q,|\mathcal{L}|_{p}^{\wedge}\right)$ is p-complete.

Proof. The second statement follows from the first, since the spaces $\left|C_{\mathcal{L}}(\rho Q)\right|$ are $p$-good (Proposition 1.12).

By Proposition 4.3 for each $\rho \in \operatorname{Hom}(Q, S)$,

$$
\operatorname{Map}\left(B Q,|\mathcal{L}|_{p}^{\wedge}\right)_{B \rho} \simeq \operatorname{Map}\left(B(\rho Q),|\mathcal{L}|_{p}^{\wedge}\right)_{\text {incl }} .
$$

This follows since, in the notation of that proposition, the connected component in the category $\mathcal{L}_{Q}$ of the object $(\rho Q, \rho)$ is isomorphic as a category to the component in $\mathcal{L}_{\rho Q}$ of the object $(\rho Q$, Id). So it suffices to prove the theorem when $Q \leq S$ and $\rho$ is the inclusion.

Case 1: Assume first that $Q$ is elementary abelian. By [La, Theorem 0.5], it suffices to show that $\Gamma_{\mathcal{L}, Q}$ induces an isomorphism

$$
T_{Q}\left(H^{*}(|\mathcal{L}|) ; \text { incl }^{*}\right) \stackrel{\cong}{\longrightarrow} H^{*}\left(\left|C_{\mathcal{L}}(Q)\right|\right) .
$$


By Theorem 5.8, $H^{*}(|\mathcal{L}|) \cong H^{*}(\mathcal{F})$ (the ring of $\mathcal{F}$-stable elements in $H^{*}(B S)$ ), and $H^{*}\left(\left|C_{\mathcal{L}}(Q)\right|\right) \cong H^{*}\left(C_{\mathcal{F}}(Q)\right)$. The isomorphism (2) now follows from Lemma 5.7.

Case 2: Now assume that $Q \triangleleft S$ and $\mathcal{F}=N_{\mathcal{F}}(Q)$. In other words, we assume that each morphism $\varphi \in \operatorname{Hom}_{\mathcal{F}}\left(P, P^{\prime}\right)$ extends to a morphism $\bar{\varphi} \in \operatorname{Hom}_{\mathcal{F}}\left(Q P, Q P^{\prime}\right)$ such that $\bar{\varphi}(Q)=Q$. Let $\mathcal{L}_{0} \subseteq \mathcal{L}$ be the full subcategory whose objects are the subgroups $P \leq S$ such that $C_{P}(Q)$ is $C_{\mathcal{F}}(Q)$-centric.

We first show that all $\mathcal{F}$-radical $\mathcal{F}$-centric subgroups of $S$ are contained in $\mathcal{L}_{0}$. To see this, assume $P$ is in $\mathcal{L}$ but not in $\mathcal{L}_{0}$, and set $P_{0}=C_{P}(Q)$ for short. Then $P_{0} \triangleleft P$, and moreover every $\mathcal{F}$-automorphism of $P$ leaves $P_{0}$ invariant (since each element of $\operatorname{Aut}_{\mathcal{F}}(P)$ extends to an automorphism of $P Q$ which leaves $Q$ invariant). Thus

$$
K \stackrel{\text { def }}{=} \operatorname{Ker}\left[\operatorname{Aut}_{\mathcal{F}}(P) \longrightarrow \operatorname{Out}\left(P_{0}\right) \times \operatorname{Aut}\left(P / P_{0}\right)\right] \triangleleft \operatorname{Aut}_{\mathcal{F}}(P),
$$

and $K$ is a $p$-subgroup by, e.g., Go, Corollary 5.3.3]. To show that $O_{p}\left(\operatorname{Out}_{\mathcal{F}}(P)\right) \neq$ 1 , and hence that $P$ is not $\mathcal{F}$-radical, it thus suffices to show that $K \not \leq \operatorname{Inn}(P)$.

Since $P_{0}$ is not in $\mathcal{L}_{0}$, it is not $C_{\mathcal{F}}(Q)$-centric. We can assume that $C_{C_{S}(Q)}\left(P_{0}\right) \not \leq$ $P_{0}$; otherwise $P$ can be replaced by another subgroup in its $C_{\mathcal{F}}(Q)$-conjugacy class for which this does hold. Set $P_{1}=C_{S}\left(Q P_{0}\right) \cdot P_{0} \supsetneqq P_{0}$, so that $1 \neq P_{1} / P_{0} \triangleleft$ $N_{S}\left(P_{0}\right) / P_{0}$. Since any nontrivial normal subgroup of a $p$-group intersects nontrivially with its center $\left(1 \neq N \triangleleft P\right.$ implies $\left.N \cap Z(P)=N^{P} \neq 1\right)$, we have

$$
P_{2} / P_{0} \stackrel{\text { def }}{=} P_{1} / P_{0} \cap Z\left(N_{S}\left(P_{0}\right) / P_{0}\right) \neq 1 .
$$

Also, $P_{2} \cap P=P_{0}$, since $P_{2} \leq C_{S}\left(P_{0} Q\right) \cdot P_{0}$ and $P_{0}=C_{P}(Q)$. In particular, for each $x \in P_{2} \backslash P_{0}, x$ normalizes $P$, its conjugation action is an inner automorphism of $P_{0}$ and the identity on $P / P_{0}$, but is not an inner automorphism of $P$ since $P$ is $\mathcal{F}$-centric and $x \notin P$. Thus $c_{x} \in K \backslash \operatorname{Inn}(P)$, and this finishes the proof that $P$ is not $\mathcal{F}$-radical.

The subcategory $\mathcal{L}_{0}$ thus contains all $\mathcal{F}$-radical subgroups of $S$ which are in $\mathcal{L}$. By Corollary 3.6, the inclusion induces a homotopy equivalence $\left|\mathcal{L}_{0}\right|_{p}^{\wedge} \simeq|\mathcal{L}|_{p}^{\wedge}$.

Let $\left(\mathcal{L}_{0}\right)_{Q \text {,Id }}$ be the category whose objects are the pairs $(P, \alpha)$ for $P$ in $\mathcal{L}_{0}$ and $\alpha \in \operatorname{Hom}_{\mathcal{F}}(Q, P)$, and where

$$
\operatorname{Mor}_{\left(\mathcal{L}_{0}\right)_{Q, \text { Id }}}\left((P, \alpha),\left(P^{\prime}, \alpha^{\prime}\right)\right)=\left\{\varphi \in \operatorname{Mor}_{\mathcal{L}}\left(P, P^{\prime}\right) \mid \alpha^{\prime}=\pi(\varphi) \circ \alpha\right\} .
$$

This is equivalent to the component, in the category $\left(\mathcal{L}_{0}\right)_{Q}$ of Proposition 4.3 of the object $(Q$, Id $)$. Hence $\left.\operatorname{Map}\left(B Q,\left|\mathcal{L}_{0}\right| \wedge\right)_{p}\right)_{\text {incl }} \simeq\left|\left(\mathcal{L}_{0}\right)_{Q, \text { Id }}\right|_{p}^{\wedge}$ by Proposition 4.3

We claim that there are functors

$$
\left(\mathcal{L}_{0}\right)_{Q, \mathrm{Id}} \stackrel{\sigma}{\longleftarrow} C_{\mathcal{L}}(Q)
$$

which are inverses up to natural transformation. To see this, for each $(P, \alpha)$ in $\left(\mathcal{L}_{0}\right)_{Q, \text { Id }}$, choose some $\bar{\alpha} \in \operatorname{Hom}_{\mathcal{F}}\left(C_{P}(Q) \cdot Q, C_{S}(Q) \cdot Q\right)$ which extends $\alpha$ (use condition (II) in Definition [1.2), set $R_{P, \alpha}=\bar{\alpha}\left(C_{P}(Q)\right)$, and let $\beta_{P, \alpha} \in \operatorname{Mor}_{\mathcal{L}}\left(R_{P, \alpha} \cdot Q, P\right)$ be any morphism such that $\pi\left(\beta_{P, \alpha}\right)=\bar{\alpha}^{-1}$. When $Q \leq P \leq C_{S}(Q) \cdot Q$ and $\alpha$ is the inclusion, we choose $R_{P, \alpha}=C_{P}(Q)$ and $\beta_{P, \alpha}$ the identity morphism of $P$ in $\mathcal{L}$. Define $\sigma$ by setting $\sigma(P, \alpha)=R_{P, \alpha}$, and by letting $\sigma(\varphi)$, for any 
$\varphi \in \operatorname{Mor}_{\left(\mathcal{L}_{0}\right)_{Q, \text { Id }}}\left((P, \alpha),\left(P^{\prime}, \alpha^{\prime}\right)\right)$, be the unique morphism which makes the following square commute in $\mathcal{L}$ :

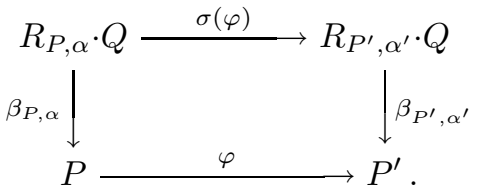

The existence and uniqueness of $\sigma(\varphi)$ follow from Lemma 1.10(a). Define $\tau$ by setting $\tau(P)=(P Q$, incl $)$ and letting

$$
\begin{aligned}
\tau_{P, P^{\prime}}: \operatorname{Mor}_{C_{\mathcal{L}}(Q)}\left(P, P^{\prime}\right) \longrightarrow \operatorname{Mor}_{\left(\mathcal{L}_{0}\right)_{Q, \text { Id }}}\left((P Q, \operatorname{incl}),\left(P^{\prime} Q, \text { incl }\right)\right) \\
\subseteq \operatorname{Mor}_{\mathcal{L}}\left(P Q, P^{\prime} Q\right)
\end{aligned}
$$

be the inclusion. Then $\sigma \circ \tau$ is the identity functor, and there is a natural transformation $\tau \circ \sigma \longrightarrow$ Id which sends each object $(P, \alpha)$ to the morphism $\beta_{P, \alpha}$.

This now shows that the composite

$$
\begin{aligned}
& \left|C_{\mathcal{L}}(Q)\right|_{p}^{\wedge} \stackrel{\tau}{\simeq}\left|\left(\mathcal{L}_{0}\right)_{Q, \mathrm{Id}}\right|_{p}^{\wedge} \stackrel{|\Phi|^{\prime}}{\simeq} \operatorname{Map}\left(B Q,\left|\mathcal{L}_{0}\right|_{p}^{\wedge}\right)_{\text {incl }} \\
& \stackrel{\text { incl }}{\simeq} \operatorname{Map}\left(B Q,|\mathcal{L}|_{p}^{\wedge}\right)_{\text {incl }}
\end{aligned}
$$

is a homotopy equivalence. By construction, it is equal to $\Gamma_{\mathcal{L}, Q}^{\prime}$.

Case 3: We now prove the general case of the theorem. Fix $Q \leq S$, and assume inductively that the theorem holds for maps with source $B Q^{\prime}$ for all $p$ groups $Q^{\prime}$ with $\left|Q^{\prime}\right|<|Q|$. We can also assume that $Q$ is fully normalized in $\mathcal{F}$. Fix a subgroup $V \leq Q \cap Z\left(N_{S}(Q)\right)=Q^{N_{S}(Q)}$ of order $p$. By Proposition A.2(b), there is $\psi \in \operatorname{Hom}_{\mathcal{F}}\left(N_{S}(V), S\right)$ such that $\psi(V)$ is fully centralized in $\mathcal{F}$. Then $N_{S}(Q) \leq C_{S}(V)$, and so $\left|N_{S}(\psi Q)\right| \geq\left|N_{S}(Q)\right|$, with equality since $Q$ is fully normalized. Upon replacing $V$ and $Q$ by their images under $\psi$, we can thus assume that $V$ is fully centralized in $\mathcal{F}$ (and $Q$ is still fully normalized in $\mathcal{F}$ ). Furthermore, since $N_{S}(Q)=N_{C_{S}(V)}(Q), Q$ is also fully normalized in $C_{\mathcal{F}}(V)$.

By Case 1, the inclusion $\left|C_{\mathcal{L}}(V)\right|_{p}^{\wedge} \subseteq|\mathcal{L}|_{p}^{\wedge}$ induces a homotopy equivalence from $\operatorname{Map}\left(B V,\left|C_{\mathcal{L}}(V)\right|_{p}^{\wedge}\right)_{\text {incl }}$ to $\operatorname{Map}\left(B V,|\mathcal{L}|_{p}^{\wedge}\right)_{\text {incl }}$; and hence a homotopy equivalence

$$
\operatorname{Map}\left(E Q / V,\left|C_{\mathcal{L}}(V)\right|_{p}^{\wedge}\right)_{\text {incl }} \longrightarrow \operatorname{Map}\left(E Q / V,|\mathcal{L}|_{p}^{\wedge}\right)_{\text {incl }}
$$

which is $Q / V$-equivariant. This remains a homotopy equivalence after taking homotopy fixed point sets, and thus restricts to a homotopy equivalence

$$
\operatorname{Map}\left(B Q,\left|C_{\mathcal{L}}(V)\right|_{p}^{\wedge}\right)_{\text {incl }} \longrightarrow \operatorname{Map}\left(B Q,|\mathcal{L}|_{p}^{\wedge}\right)_{\text {incl }} .
$$

So we can assume that $\mathcal{L}=C_{\mathcal{L}}(V)$, i.e., that $V$ is central in $\mathcal{L}$.

We recall the notation of Lemma 5.6. Let $\mathcal{F} / V$ be the induced fusion system over $S / V$. Let $\mathcal{L} / V$ be the category whose objects are the subgroups $P / V \leq S / V$ such that $P$ is $\mathcal{F}$-centric, and where

$$
\operatorname{Mor}_{\mathcal{L} / V}(P / V, Q / V)=\operatorname{Mor}_{\mathcal{L}}(P, Q) / \delta_{P}(V) .
$$

Let $\mathcal{L}_{0} \subseteq \mathcal{L}$ and $(\mathcal{L} / V)^{c} \subseteq \mathcal{L} / V$ be the full subcategories whose objects are those $P \leq S$ and $P / V \leq S / V$, respectively, such that $P / V$ is $(\mathcal{F} / V)$-centric. Set $\mathcal{F}^{\prime}=$ $N_{\mathcal{F}}(Q)$ and $\mathcal{L}^{\prime}=N_{\mathcal{L}}(Q)$; and define $\mathcal{L}^{\prime} / V, \mathcal{L}_{0}^{\prime} \subseteq \mathcal{L}^{\prime}$, and $\left(\mathcal{L}^{\prime} / V\right)^{c} \subseteq \mathcal{L}^{\prime} / V$ in a similar way. 
By Lemma 5.6 there are fibrations

$$
B V \longrightarrow\left|\mathcal{L}_{0}\right|_{p}^{\wedge} \stackrel{f}{\longrightarrow}\left|(\mathcal{L} / V)^{c}\right|_{p}^{\wedge} \quad \text { and } \quad B V \longrightarrow\left|\mathcal{L}_{0}^{\prime}\right|_{p}^{\wedge} \stackrel{f^{\prime}}{\longrightarrow}\left|\left(\mathcal{L}^{\prime} / V\right)^{c}\right|_{p}^{\wedge},
$$

and homotopy equivalences $\left|\mathcal{L}_{0}\right|_{p}^{\wedge} \simeq|\mathcal{L}|_{p}^{\wedge}$ and $\left|\mathcal{L}_{0}^{\prime}\right|_{p}^{\wedge} \simeq\left|\mathcal{L}^{\prime}\right|_{p}^{\wedge}$. We thus get a homotopy pullback square of mapping spaces

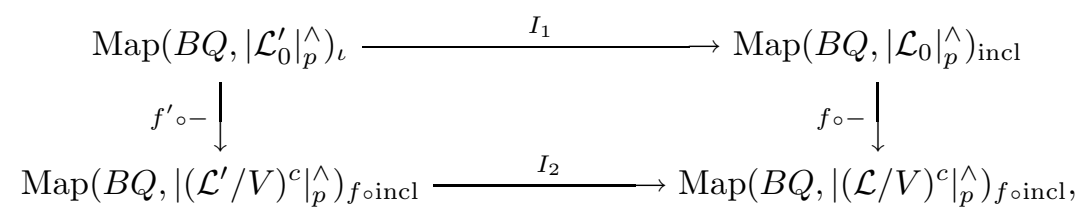

where $\operatorname{Map}\left(B Q,\left|\mathcal{L}_{0}^{\prime}\right|_{p}^{\wedge}\right)_{\iota}$ is the union of the connected components which map to the inclusion in $\left|\mathcal{L}_{0}\right|_{p}^{\wedge}$ and to $f \circ$ incl in $\left|\left(\mathcal{L}^{\prime} / V\right)^{c}\right|_{p}^{\wedge}$, and where $I_{1}$ and $I_{2}$ are inclusions. By (1), together with the induction hypothesis, there are homotopy equivalences

$$
\begin{aligned}
\left|C_{(\mathcal{L} / V)^{c}}(Q / V)\right|_{p}^{\wedge} \stackrel{\Gamma_{\mathcal{L} / V, Q / V}^{\prime}}{\simeq} \operatorname{Map}\left(B(Q / V),\left|(\mathcal{L} / V)^{c}\right|_{p}^{\wedge}\right)_{\text {incl }} \\
\stackrel{- \text { oproj }}{\simeq} \operatorname{Map}\left(B Q,\left|(\mathcal{L} / V)^{c}\right|_{p}^{\wedge}\right)_{f \text { oincl }},
\end{aligned}
$$

and similarly for maps to $\left|\left(\mathcal{L}^{\prime} / V\right)^{c}\right|_{p}^{\wedge}$. Since by definition, $\Gamma_{\mathcal{L} / V, Q / V}^{\prime}$ is the composite of $\Gamma_{\mathcal{L}^{\prime} / V, Q / V}^{\prime}$ with the inclusion, this shows that the map $I_{2}$ in the above diagram is a homotopy equivalence. Thus $I_{1}$ is also a homotopy equivalence. In particular, $\operatorname{Map}\left(B Q,\left|\mathcal{L}_{0}^{\prime}\right|_{p}^{\wedge}\right)_{\iota}$ is connected, and hence contains only the component of the inclusion. The theorem now follows from Case 2, when applied to the mapping space $\operatorname{Map}\left(B Q,\left|\mathcal{L}_{0}^{\prime}\right|_{p}^{\wedge}\right)_{\text {incl }}$.

\section{A TOPOLOGICAL CHARACTERIZATION}

In Definition 1.8, we defined the classifying space of a $p$-local finite group $(S, \mathcal{F}, \mathcal{L})$ to be the space $|\mathcal{L}|_{p}^{\wedge}$. In this section, we show that the triple $(S, \mathcal{F}, \mathcal{L})$ is in fact determined up to isomorphism by the homotopy type of $|\mathcal{L}|_{p}^{\wedge}$ (Theorem 7.4). Afterwards, we prove a more intrinsic characterization of these spaces, by showing that a $p$-complete space $X$ is the classifying space of some $p$-local finite group if and only if it satisfies certain conditions listed in Theorem 7.5

Definition 7.1. For any space $X$, any $p$-group $S$, and any map $f: B S \longrightarrow X$, define $\mathcal{F}_{S, f}(X)$ to be the category whose objects are the subgroups of $S$, and whose morphisms are given by

$$
\operatorname{Hom}_{\mathcal{F}_{S, f}(X)}(P, Q)=\left\{\left.\varphi \in \operatorname{Inj}(P, Q)|f|_{B P} \simeq f\right|_{B Q} \circ B \varphi\right\}
$$

for each $P, Q \leq S$.

The category $\mathcal{F}_{S, f}(X)$ is clearly a fusion system over $S$, but is not in general saturated.

We next consider the linking system of a space. Let $\pi(Z)$ denote the fundamental groupoid of a space $Z$. If $H$ is a path in $Z$, then $[H]$ will denote its homotopy class relative to endpoints, regarded as a morphism in $\pi(Z)$. We regard $\pi(Z)$ as a discrete category.

Definition 7.2. For any space $X$, any $p$-group $S$, and any map $f: B S \longrightarrow X$, define categories $\mathcal{L}_{S, f}^{c}(X)$ and $\widehat{\mathcal{L}}_{S, f}^{c}(X)$ as follows. The objects of $\mathcal{L}_{S, f}^{c}(X)$ are the 
$\mathcal{F}_{S, f}(X)$-centric subgroups of $S$, while the objects of $\widehat{\mathcal{L}}_{S, f}^{c}(X)$ are the pairs $(P, \alpha)$ such that $P \leq S$ is $\mathcal{F}_{S, f}(X)$-centric and $B \alpha \simeq f \circ B \varphi$ for some $\varphi \in \operatorname{Inj}(P, S)$. Morphisms in $\widehat{\mathcal{L}}_{S, f}^{c}(X)$ are defined by

$$
\begin{aligned}
\operatorname{Mor}_{\widehat{\mathcal{L}}_{S, f}^{c}(X)}((P, \alpha),(Q, \beta)) & \\
& =\left\{(\varphi,[H]) \mid \varphi \in \operatorname{Inj}(P, Q),[H] \in \operatorname{Mor}_{\pi(\operatorname{Map}(B P, X))}(\alpha, \beta \circ B \varphi)\right\},
\end{aligned}
$$

while morphisms in $\mathcal{L}_{S, f}^{c}$ are given by

$$
\operatorname{Mor}_{\mathcal{L}_{S, f}^{c}}(P, Q)=\operatorname{Mor}_{\widehat{\mathcal{L}}_{S, f}^{c}}\left(\left(P,\left.f\right|_{B P}\right),\left(Q,\left.f\right|_{B Q}\right)\right) .
$$

In particular, we can regard $\mathcal{L}_{S, f}^{c}$ as a full subcategory of $\widehat{\mathcal{L}}_{S, f}^{c}$ by identifying an object $P$ in $\mathcal{L}_{S, f}^{c}$ with $\left(P,\left.f\right|_{P}\right)$ in $\widehat{\mathcal{L}}_{S, f}^{c}$. Since every object in $\widehat{\mathcal{L}}_{S, f}^{c}$ is isomorphic to an object in the subcategory $\mathcal{L}_{S, f}^{c}$, this inclusion is an equivalence of categories. The goal is to show that in certain situations, $\mathcal{L}_{S, f}^{c}$ is a centric linking system associated to $\mathcal{F}_{S, f}$. We define $\widehat{\mathcal{L}}_{S, f}^{c}$ because certain constructions are more natural when working in this larger category.

In BLO, Definition 2.5], we defined categories $\mathcal{L}_{p}^{c}(X) \subseteq \mathcal{L}_{p}(X)$, for any space $X$, whose objects are certain pairs $(P, \alpha)$ such that $P$ is a $p$-group and $\alpha: B P \longrightarrow X$ a map. Upon comparing these two definitions, we see that whenever $f$ is a "homotopy monomorphism" in the sense of [BLO, Definition 2.2], then $\widehat{\mathcal{L}}_{S, f}^{c}(X)$ is a full subcategory of $\mathcal{L}_{p}(X)$. When $X=|\mathcal{L}|_{p}^{\wedge}$ for some $p$-local finite group $(S, \mathcal{F}, \mathcal{L})$ and $f$ is the inclusion, then it turns out that the inclusion $\widehat{\mathcal{L}}_{S, f}^{c}(X) \subseteq \mathcal{L}_{p}^{c}(X)$ is an equivalence of categories. We prefer to work here with the smaller category $\widehat{\mathcal{L}}_{S, f}^{c}(X)$, to a large extent to avoid having to deal with questions involving definitions and properties of homotopy monomorphisms.

Let $(S, \mathcal{F}, \mathcal{L})$ be a $p$-local finite group, and let $\pi: \mathcal{L} \longrightarrow \mathcal{F}^{c}$ be the projection functor. For each $P \leq S$, let

$$
\theta_{P}: \mathcal{B}(P) \longrightarrow \mathcal{L}
$$

be the functor which sends $o_{P}$ to $P$ and sends a morphism $\check{g}$ (for $g \in P$ ) to $\widehat{g} \in \operatorname{Aut}_{\mathcal{L}}(P)$. We write

$$
\theta=\left|\theta_{S}\right|_{p}^{\wedge}: B S=|\mathcal{B}(S)| \stackrel{|\theta|_{p}^{\wedge}}{\longrightarrow}|\mathcal{L}|_{p}^{\wedge}
$$

for short. For each $\varphi \in \operatorname{Hom}_{\mathcal{L}}(P, Q)$, let

$$
\eta_{\varphi}: \theta_{P} \longrightarrow \theta_{Q} \circ \pi_{P, Q}(\varphi)
$$

be the natural transformation of functors $\mathcal{B}(P) \longrightarrow \mathcal{L}$ which sends the object $o_{P}$ to the morphism $\varphi$.

Now define functors

$$
\xi_{\mathcal{F}}: \mathcal{F} \longrightarrow \mathcal{F}_{S, \theta}\left(|\mathcal{L}|_{p}^{\wedge}\right) \quad \text { and } \quad \xi_{\mathcal{L}}: \mathcal{L} \longrightarrow \widehat{\mathcal{L}}_{S, \theta}^{c}\left(|\mathcal{L}|_{p}^{\wedge}\right)
$$

as follows. On objects, for all $P \leq S$,

$$
\xi_{\mathcal{F}}(P)=P \quad \text { and } \quad \xi_{\mathcal{L}}(P)=\left(P,\left|\theta_{P}\right|_{p}^{\wedge}\right) .
$$

For each $\varphi \in \operatorname{Hom}_{\mathcal{F}}(P, Q)$,

$$
\xi_{\mathcal{F}}(\varphi)=\varphi
$$


while for each morphism $\varphi \in \operatorname{Mor}_{\mathcal{L}}(P, Q)$,

$$
\xi_{\mathcal{L}}(\varphi)=\left(\pi_{P, Q}(\varphi),\left[\left|\eta_{\varphi}\right|\right]\right),
$$

where $\left|\eta_{\varphi}\right|$ is regarded as a homotopy $B P \times I \longrightarrow|\mathcal{L}|_{p}^{\wedge}$. Note that in the definition of $\xi_{\mathcal{L}}$ we are assuming that each $\mathcal{F}$-centric subgroup of $S$ is also $\mathcal{F}_{S, \theta}\left(|\mathcal{L}|_{p}^{\wedge}\right)$-centric; this will be shown in the next theorem.

Proposition 7.3. The following hold for any p-local finite group $(S, \mathcal{F}, \mathcal{L})$.

(a) The functor $\xi_{\mathcal{F}}: \mathcal{F} \longrightarrow \mathcal{F}_{S, \theta}\left(|\mathcal{L}|_{p}^{\wedge}\right)$ is an isomorphism of categories.

(b) The functor $\xi_{\mathcal{L}}: \mathcal{L} \longrightarrow \widehat{\mathcal{L}}_{S, \theta}^{c}\left(|\mathcal{L}|_{p}^{\wedge}\right)$ is an equivalence of categories.

Proof. We keep the above notation; in particular, $\theta=\left|\theta_{S}\right|_{p}^{\wedge}: B S \longrightarrow|\mathcal{L}|_{p}^{\wedge}$. For all $P \leq S$, let $i_{P} \in \operatorname{Inj}(P, S)$ be the inclusion, and note that $\theta \circ B i_{P} \simeq\left|\theta_{P}\right|_{p}^{\wedge}$.

By Corollary 4.5, for all $P, Q \leq S$,

$$
\begin{aligned}
& \operatorname{Mor}_{\mathcal{F}_{S, \theta}\left(|\mathcal{L}|_{p}\right)}(P, Q) \cong\left\{\left.\varphi \in \operatorname{Inj}(P, Q)|\theta|_{B P} \simeq \theta\right|_{B Q} \circ B \varphi\right\} \\
& \quad=\left\{\varphi \in \operatorname{Inj}(P, Q) \mid \exists \psi \in \operatorname{Hom}_{\mathcal{F}}(P, S), \psi=i_{Q} \circ \varphi\right\}=\operatorname{Hom}_{\mathcal{F}}(P, Q) .
\end{aligned}
$$

So $\xi_{\mathcal{F}}$ induces bijections on morphism sets, and is thus an equivalence of categories.

In particular, this shows that a subgroup $P \leq S$ is $\mathcal{F}$-centric if and only if it is $\mathcal{F}_{S, \theta}\left(|\mathcal{L}|_{p}^{\wedge}\right)$-centric, and thus that $\xi_{\mathcal{L}}$ is bijective on isomorphism classes of objects. It remains to show that $\xi_{\mathcal{L}}$ induces bijections on morphism sets. Fix a pair of $\mathcal{F}$-centric subgroups $P, Q \leq S$, and consider the following commutative diagram:

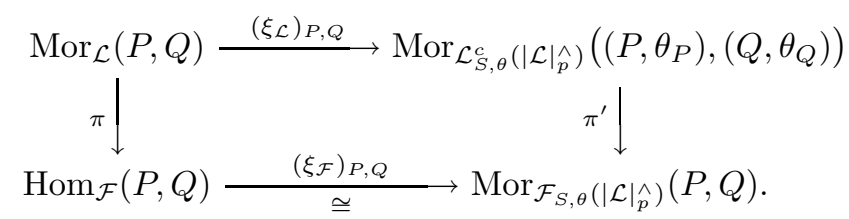

Here, $\pi^{\prime}$ is the "forgetful" functor which is the identity on objects and sends a morphism $(\alpha,[H])$ to $\alpha$. Since $P$ is $\mathcal{F}$-centric, $\pi_{1}\left(\operatorname{Map}\left(B P,|\mathcal{L}|_{p}^{\wedge}\right)_{\left|\theta_{P}\right|}\right) \cong Z(P)$, and hence $\pi^{\prime}$ is the orbit map of a free action of $Z(P)$ on $\operatorname{Mor}_{\mathcal{L}_{S, \theta}^{c}(|\mathcal{L}| \wedge)}\left(\left(P, \theta_{P}\right),\left(Q, \theta_{Q}\right)\right)$ (by definition of $\mathcal{L}_{S, \theta}^{c}(-)$ ). By condition (A), $\pi$ is the orbit map of a free $Z(P)$ action on $\operatorname{Mor}_{\mathcal{L}}(P, Q)$. Also, $\left(\xi_{\mathcal{L}}\right)_{P, Q}$ is easily checked to be $Z(P)$-equivariant, and hence is a bijection since the orbit map $\left(\xi_{\mathcal{F}}\right)_{P, Q}$ is a bijection.

Define an isomorphism $(S, \mathcal{F}, \mathcal{L}) \longrightarrow\left(S^{\prime}, \mathcal{F}^{\prime}, \mathcal{L}^{\prime}\right)$ of $p$-local finite groups to consist of a triple $\left(\alpha, \alpha_{\mathcal{F}}, \alpha_{\mathcal{L}}\right)$, where

$$
S \stackrel{\alpha}{\longrightarrow} S^{\prime}, \quad \mathcal{F} \stackrel{\alpha_{\mathcal{F}}}{\longrightarrow} \mathcal{F}^{\prime}, \quad \text { and } \quad \mathcal{L} \stackrel{\alpha_{\mathcal{L}}}{\longrightarrow} \mathcal{L}^{\prime}
$$

are isomorphisms of groups and categories such that $\alpha_{\mathcal{F}}(P)=\alpha_{\mathcal{L}}(P)=\alpha(P)$ for all $P \leq S$, and such that they commute in the obvious way with the projections $\mathcal{L} \longrightarrow \mathcal{F}$ and the structure maps $P \longrightarrow \operatorname{Aut}_{\mathcal{L}}(P)$.

Theorem 7.4. If $(S, \mathcal{F}, \mathcal{L})$ and $\left(S^{\prime}, \mathcal{F}^{\prime}, \mathcal{L}^{\prime}\right)$ are two -local finite groups such that $|\mathcal{L}|_{p}^{\wedge} \simeq\left|\mathcal{L}^{\prime}\right|_{p}^{\wedge}$, then $(S, \mathcal{F}, \mathcal{L})$ and $\left(S^{\prime}, \mathcal{F}^{\prime}, \mathcal{L}^{\prime}\right)$ are isomorphic as p-local finite groups. Thus the p-local finite group $(S, \mathcal{F}, \mathcal{L})$ is determined by the homotopy type of $|\mathcal{L}|_{p}^{\wedge}$. 
Proof. If $|\mathcal{L}|_{p}^{\wedge} \stackrel{f}{\simeq}\left|\mathcal{L}^{\prime}\right|_{p}^{\wedge}$ is a homotopy equivalence, then by Theorem $4.4(\mathrm{a}, \mathrm{b})$, there is $\rho \in \operatorname{Iso}\left(S, S^{\prime}\right)$ such that the square

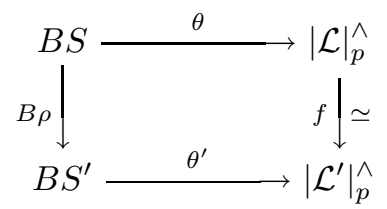

commutes up to homotopy. These thus induce isomorphisms of categories

$$
\mathcal{F}_{S, \theta}\left(|\mathcal{L}|_{p}^{\wedge}\right) \cong \mathcal{F}_{S^{\prime}, \theta^{\prime}}\left(\left|\mathcal{L}^{\prime}\right|_{p}^{\wedge}\right) \quad \text { and } \quad \mathcal{L}_{S, \theta}^{c}\left(|\mathcal{L}|_{p}^{\wedge}\right) \cong \mathcal{L}_{S^{\prime}, \theta^{\prime}}^{c}\left(\left|\mathcal{L}^{\prime}\right|_{p}^{\wedge}\right),
$$

and so $(S, \mathcal{F}, \mathcal{L}) \cong\left(S^{\prime}, \mathcal{F}^{\prime}, \mathcal{L}^{\prime}\right)$ by Proposition 7.3 .

The next theorem provides a characterization of classifying spaces of $p$-local finite groups. Recall that a map $f: X \longrightarrow Y$ between spaces is centric if the induced map

$$
\operatorname{Map}(X, X)_{\text {Id }} \stackrel{f_{\circ}-}{\longrightarrow} \operatorname{Map}(X, Y)_{f}
$$

is a homotopy equivalence.

Theorem 7.5. A p-complete space $X$ is the classifying space of some p-local finite group if and only if there are a p-group $S$ and a map $f: B S \longrightarrow X$ such that

(a) the fusion system $\mathcal{F}_{S, f}(X)$ is saturated,

(b) there is a homotopy equivalence $X \simeq\left|\mathcal{L}_{S, f}^{c}(X)\right|_{p}^{\wedge}$, and

(c) $\left.f\right|_{B P}$ is a centric map for each $\mathcal{F}_{S, f}(X)$-centric subgroup $P \leq S$.

When these hold, $\mathcal{L}_{S, f}^{c}(X)$ is a centric linking system associated to $\mathcal{F}_{S, f}(X)$.

Proof. Assume first that $X=|\mathcal{L}|_{p}^{\wedge}$, where $\mathcal{L}$ is a centric linking system associated to a fusion system $\mathcal{F}$ over a $p$-group $S$. Then if $f$ denotes the inclusion $B S \subseteq|\mathcal{L}|_{p}^{\wedge} \simeq X$, condition (a) holds since $\mathcal{F}_{S}(X) \cong \mathcal{F}$ by Proposition 7.3 (a), condition (b) follows from Proposition 7.3 b), and condition (c) holds by Theorem 4.4(c).

Now assume that $X$ is a $p$-complete space, and that conditions $(\mathrm{a}-\mathrm{c}$ ) hold for some map $B S \stackrel{f}{\longrightarrow} X$. Set $\mathcal{F}=\mathcal{F}_{S, f}(X)$ and $\mathcal{L}=\mathcal{L}_{S, f}^{c}(X)$ for short. In particular, $\mathcal{F}$ is saturated by (a), and $X \simeq|\mathcal{L}|_{p}^{\wedge}$ by (b). We will show that $\mathcal{L}$ is a centric linking system associated to $\mathcal{F}$, and thus that $(S, \mathcal{F}, \mathcal{L})$ is a $p$-local finite group.

Define maps $P \stackrel{\delta_{P}}{\longrightarrow} \operatorname{Aut}_{\mathcal{L}}(P)$ by sending $g \in P$ to the pair $\left(c_{g},\left[H_{g}\right]\right)$, where $H_{g}$ is the homotopy

$$
H_{g}: B P \times I \stackrel{\left|\eta_{g}\right|}{\longrightarrow} B P \subseteq B S \stackrel{f}{\longrightarrow} X
$$

induced by the natural transformation of functors Id $\stackrel{\eta_{g}}{\longrightarrow} c_{g}$ which sends the object $o_{P}$ in $\mathcal{B}(P)$ to the morphism $\check{g}$ of $\mathcal{B}(P)$. Condition $(\mathrm{B})$ is clear. By condition (c), for each $P \in \operatorname{Ob}(\mathcal{L}),\left.f\right|_{B P}$ is a centric map, and thus

$$
\operatorname{Map}(B P, X)_{\left.f\right|_{B P}} \simeq \operatorname{Map}(B P, B P)_{\mathrm{Id}} \simeq B Z(P) .
$$

This proves condition $(\mathrm{A})$. 
Condition $(\mathrm{C})$ means showing, for each $(\varphi,[H]) \in \operatorname{Mor}_{\mathcal{L}}(P, Q)$ and each $g \in P$, that the following square commutes:

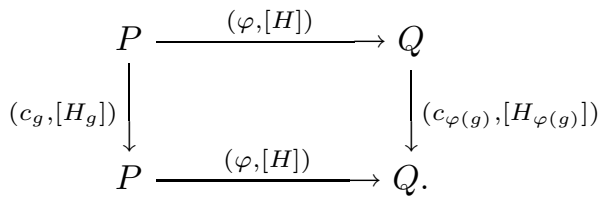

Here $[H]$ is the homotopy class of a path $H$ in $\operatorname{Map}(B P, X)$. Clearly, $\varphi \circ c_{g}=c_{\varphi(g)} \circ \varphi$. It remains to check that the following two paths in $\operatorname{Map}(B P, X)$ are homotopic:

$$
\left.\left.\left.f\right|_{B P} \stackrel{H}{\longrightarrow} f\right|_{B Q} \circ B \varphi \stackrel{H_{\varphi(g)} \circ B \varphi}{\longrightarrow} f\right|_{B Q} \circ B\left(\varphi \circ c_{g}\right)
$$

and

$$
\left.\left.\left.f\right|_{B P} \stackrel{H_{g}}{\longrightarrow} f\right|_{B P} \circ B c_{g} \stackrel{H \circ B c_{g}}{\longrightarrow} f\right|_{B Q} \circ B\left(\varphi \circ c_{g}\right) .
$$

The map

$$
F: B P \times I \times I \longrightarrow X \quad \text { defined by } \quad F(x, s, t)=H\left(\left|\eta_{g}\right|(x, t), s\right)
$$

is such a homotopy, since

$$
\begin{aligned}
& F(x, s, 0)=H(x, s), \\
& F(x, 1, t)=f \circ B \varphi \circ\left|\eta_{g}\right|(x, t)=H_{\varphi(g)}(B \varphi(x), t), \\
& F(x, 0, t)=f \circ\left|\eta_{g}\right|(x, t)=H_{g}(x, t) \text { and } \\
& F(x, s, 1)=H\left(B c_{g}(x), s\right) .
\end{aligned}
$$

Note that when $X=|\mathcal{L}|_{p}^{\wedge}$ for some $p$-local finite group $(S, \mathcal{F}, \mathcal{L})$, then the choice of the map $f: B S^{\prime} \longrightarrow X$ which satisfies conditions (a) and (c) is essentially unique. By Theorem 4.4(a), any such $f$ is homotopic to the composite $B S^{\prime} \stackrel{B \varphi}{\longrightarrow} B S \stackrel{\theta}{\longrightarrow} X$ for some $\varphi \in \operatorname{Hom}\left(S^{\prime}, S\right)$, where $\theta$ denotes the canonical inclusion. Then $\varphi$ is a monomorphism by (c), since otherwise $f$ factors through $B\left(S^{\prime} / R\right)$ for some $1 \neq$ $R \triangleleft S^{\prime}$, and this is impossible when $f$ is a centric map. One also shows, using (c), that $\varphi\left(S^{\prime}\right)$ is centric in $S$. Since $\mathcal{F}_{S^{\prime}, f}(X)$ is saturated by (a), $\operatorname{Out}_{\mathcal{F}_{S^{\prime}, f}(X)}\left(S^{\prime}\right)$ has

order prime to $p$; hence $N_{S}\left(\varphi\left(S^{\prime}\right)\right)=1$ (this is a subgroup of $\operatorname{Out}_{\mathcal{F}_{S^{\prime}, f}(X)}\left(S^{\prime}\right)$ by Theorem 4.4 and the centricity of $\left.\varphi\left(S^{\prime}\right)\right)$, and thus $\varphi$ is surjective.

By the above proof, conditions (a) and (c) in Theorem 7.5 imply that $X$ and $f$ determine a $p$-local finite group $\left(S, \mathcal{F}_{S, f}(X), \mathcal{L}_{S, f}^{c}(X)\right)$. Condition (b) then tells us that $X$ is its classifying space. The three conditions (a,b,c) thus imply that $f$ is essentially unique.

In a later paper, we will show that condition (a) can be replaced by the condition that $B S \stackrel{f}{\longrightarrow} X$ is "Sylow", in the sense that any map $B P \longrightarrow X$ (for a $p$-group $P$ ) factors through $f$.

\section{SpaCes of Self EQUivalenCES}

We next describe the monoid $\operatorname{Aut}\left(|\mathcal{L}|_{p}^{\wedge}\right)$ of self homotopy equivalences of $|\mathcal{L}|_{p}^{\wedge}$. Understanding this space is essential when constructing fibrations with fiber the classifying space of a $p$-local finite group, although such constructions will not be discussed in this paper.

We first recall some definitions from $\mathrm{BLO}$. For any space $X$, Aut $(X)$ denotes the monoid of self homotopy equivalences of $X, \operatorname{Out}(X)=\pi_{0}(\operatorname{Aut}(X))$ is the group 
of homotopy classes of equivalences, and $\mathcal{A} u t(X)$ is the fundamental groupoid of $\operatorname{Aut}(X)$. Also, for any discrete category $\mathcal{C}, \mathcal{A} u t(\mathcal{C})$ is the category whose objects are the self equivalences of $\mathcal{C}$ and whose morphisms are the natural isomorphisms between self equivalences, and $\operatorname{Out}(\mathcal{C})=\pi_{0}(|\mathcal{A} u t(\mathcal{C})|)$ is the group of isomorphism classes of self equivalences. Both $\mathcal{A} u t(X)$ and $\mathcal{A} u t(\mathcal{C})$ are discrete categories. They are also strict monoidal categories, in the sense that composition defines a strictly associative functor

$$
\mathcal{A} u t(-) \times \mathcal{A} u t(-) \longrightarrow \mathcal{A} u t(-)
$$

with strict identity. The nerve of each of these categories $\mathcal{A} u t(-)$ is thus a simplicial monoid, and its realization $|\mathcal{A} u t(-)|$ is a topological monoid.

Recall that part of the structure of a centric linking system $\mathcal{L}$ associated to a fusion system is a homomorphism $P \stackrel{\delta_{P}}{\longrightarrow} \operatorname{Aut}_{\mathcal{L}}(P)$ for each $P$ in $\mathcal{L}$. We write $P_{\delta}=\operatorname{Im}\left(\delta_{P}\right)$, which we think of as a "distinguished subgroup" of $\operatorname{Aut}_{\mathcal{L}}(P)$ which can be identified with $P$. For the purposes of this paper, an equivalence of categories $\mathcal{L} \stackrel{\psi}{\longrightarrow} \mathcal{L}$ will be called isotypical if for each $P, \psi_{P, P}$ sends the subgroup $P_{\delta} \leq \operatorname{Aut}_{\mathcal{L}}(P)$ to the subgroup $\psi P_{\delta} \leq \operatorname{Aut}_{\mathcal{L}}(\psi P)$. (This will be seen in Lemma 8.2 to be equivalent to the definition in [BLO].) Let $\mathcal{A} u t_{\text {typ }}(\mathcal{L})$ be the full subcategory of $\mathcal{A} u t(\mathcal{L})$ whose objects are the isotypical equivalences, and set $\operatorname{Out}_{\text {typ }}(\mathcal{L})=$ $\pi_{0}\left(\left|\mathcal{A} u t_{\text {typ }}(\mathcal{L})\right|\right)$.

Clearly, any equivalence which is naturally isomorphic to an isotypical equivalence is itself isotypical, and any inverse to an isotypical equivalence (inverse up to natural isomorphism of functors) is also isotypical. The subcategory $\mathcal{A} u t_{\text {typ }}(\mathcal{L})$ is thus a union of connected components of $\mathcal{A} u t(\mathcal{L})$, and $\operatorname{Out}_{\text {typ }}(\mathcal{L})$ is a subgroup of $\operatorname{Out}(\mathcal{L})$.

The main result of this section is the following theorem:

Theorem 8.1. Fix a p-local finite group $(S, \mathcal{F}, \mathcal{L})$. Then $\operatorname{Aut}\left(|\mathcal{L}|_{p}^{\wedge}\right)$ and $\left|\mathcal{A} u t_{\text {typ }}(\mathcal{L})\right|$ are equivalent as topological monoids in the sense that their classifying spaces are homotopy equivalent. In particular,

$$
\operatorname{Out}\left(|\mathcal{L}|_{p}^{\wedge}\right) \cong \operatorname{Out}_{\text {typ }}(\mathcal{L}) \quad \text { and } \quad \pi_{i}\left(\operatorname{Aut}\left(|\mathcal{L}|_{p}^{\wedge}\right)\right) \cong \begin{cases}{\underset{\mathcal{O}^{c}(\mathcal{F})}{0}(\mathcal{Z})}^{\text {if } i=1,} \\ 0 & \text { if } i \geq 2 .\end{cases}
$$

Throughout the rest of the section, we fix a $p$-local finite group $(S, \mathcal{F}, \mathcal{L})$, and let $\mathcal{L} \stackrel{\pi}{\longrightarrow} \mathcal{F}^{c}$ denote the canonical projection. For any morphism $\alpha$ in $\mathcal{L}$, we set $[\alpha]=\pi(\alpha)$ for short. The first part of the proof of Theorem 8.1 (Lemmas 8.2 and 8.3) follows closely the proof in [BLO] of the analogous result for $\operatorname{Aut}\left(B G_{p}^{\wedge}\right)$.

Lemma 8.2. Let $F: \mathcal{L} \longrightarrow \mathrm{Gr}$ denote the forgetful functor. Then for any equivalence $\mathcal{L} \stackrel{\psi}{\longrightarrow} \mathcal{L}, \psi$ is isotypical if and only if there is a natural isomorphism $F \stackrel{\Psi}{\cong} F \circ \psi$ of functors $\mathcal{L} \longrightarrow$ Gr. Also, if $\psi$ is isotypical, and if $\psi_{P}: P \longrightarrow \psi P$ denotes the restriction of $\psi_{P, P}$ under the identifications $P=P_{\delta}$ and $\psi P=\psi P_{\delta}$, then $\left(P \mapsto \psi_{P}\right)$ is a natural isomorphism of functors $F \stackrel{\cong}{\longrightarrow} F \circ \psi$.

Proof. To simplify notation, we write $P^{\prime}=\psi P$ for any $P$ in $\mathcal{L}$. Assume first that there is a natural isomorphism $\Psi: F \stackrel{\cong}{\longrightarrow} F \circ \psi$ of functors. Fix $P$, let $g \in P$ be any element, and set $\alpha=\psi_{P, P}(\widehat{g}) \in \operatorname{Aut}_{\mathcal{L}}\left(P^{\prime}\right)$. Then $\Psi$ sends $P$ to an isomorphism 
$\Psi_{P} \in \operatorname{Iso}\left(P, P^{\prime}\right)$ of groups, and

$$
c_{\Psi_{P}(g)} \circ \Psi_{P}=\Psi_{P} \circ c_{g}=[\alpha] \circ \Psi_{P}
$$

the first equality holds when $\Psi_{P}$ is replaced by any homomorphism $P \longrightarrow P^{\prime}$, and the second holds by the naturality of $\Psi$ with respect to $(P \stackrel{\widehat{g}}{\longrightarrow} P)$. Thus $[\alpha]=c_{\Psi_{P}(g)}$, so $\alpha \stackrel{\text { def }}{=} \psi_{P, P}(g)=\widehat{x}$ for some $x \in P^{\prime}$ such that $x^{-1} \Psi_{P}(g) \in Z\left(P^{\prime}\right)$. In particular, $\psi_{P, P}(\widehat{g}) \in P_{\delta}^{\prime}$, and thus $\psi_{P, P}\left(P_{\delta}\right) \leq P_{\delta}^{\prime}$. Equality now holds since the distinguished subgroups are abstractly isomorphic (and $\psi_{P, P}$ is an isomorphism).

Now assume that $\psi_{P, P}\left(P_{\delta}\right)=P_{\delta}^{\prime}$ for each $P$, and let $\psi_{P}: P \stackrel{\cong}{\longrightarrow} P^{\prime}$ be the restriction of $\psi_{P, P}$ under the identifications $P \cong P_{\delta}$ and $P^{\prime} \cong P_{\delta}^{\prime}$. We must show that $\left(P \mapsto \psi_{P}\right)$ is natural as an isomorphism of functors $F \longrightarrow F \circ \psi$, i.e., that

$$
\psi_{Q} \circ[\alpha]=[\beta] \circ \psi_{P} \in \operatorname{Hom}\left(P, Q^{\prime}\right)
$$

for any morphism $P \stackrel{\alpha}{\longrightarrow} Q$ in $\mathcal{L}$, where $\beta=\psi_{P, Q}(\alpha)$. Fix $g \in P$ and set $h=[\alpha](g)$; then $\psi$ sends $\alpha \circ \widehat{g}=\widehat{h} \circ \alpha$ in $\mathcal{L}$ to

$$
\beta \circ \widehat{\psi_{P}(g)}=\widehat{\psi_{Q}(h)} \circ \beta \in \operatorname{Mor}_{\mathcal{L}}\left(P^{\prime}, Q^{\prime}\right) .
$$

Upon comparing this with condition $(\mathrm{C})$ (and the uniqueness property shown in Lemma 1.10(b)), we see that

$$
\psi_{Q}([\alpha](g))=\psi_{Q}(h)=[\beta]\left(\psi_{P}(g)\right)
$$

for all $g \in P$, and this proves (1).

From now on, for any isotypical equivalence $\mathcal{L} \underset{\sim}{\stackrel{\psi}{\simeq}} \mathcal{L}$ and any $\mathcal{F}$-centric $P \leq S$, we let $\psi_{P}: P \stackrel{\cong}{\longrightarrow} \psi P$ denote the isomorphism obtained by restricting $\psi_{P, P}$.

Recall the category $\widehat{\mathcal{L}}_{S, f}^{c}(X)$, defined in Section 7 for any space $X$, any $p$ group $S$, and any map $f: B S \longrightarrow X$. An object in $\widehat{\mathcal{L}}_{S, f}^{c}(X)$ is a pair $(P, \alpha)$ such that $P \leq S$ is an $\mathcal{F}_{S, f}(X)$-centric subgroup of $X$ and $B P \stackrel{\alpha}{\longrightarrow} X$ is homotopic to $f \circ B \varphi$ for some $\varphi \in \operatorname{Inj}(P, S)$. A morphism in $\widehat{\mathcal{L}}_{S, f}^{c}(X)$ from $(P, \alpha)$ to $(Q, \beta)$ is a pair $(\varphi,[H])$, where $\varphi \in \operatorname{Hom}(P, Q)$ and $[H]$ is a homotopy class (relative to its endpoints) of paths in $\operatorname{Map}(B P, X)$ from $\alpha$ to $\beta \circ B \varphi$.

Let $\theta: B S \longrightarrow|\mathcal{L}|_{p}^{\wedge}$ denote the canonical inclusion. We next define functors

$$
\mathcal{A} u t_{\text {typ }}(\mathcal{L}) \stackrel{R}{\longrightarrow} \mathcal{A} u t\left(|\mathcal{L}|_{p}^{\wedge}\right) \stackrel{L}{\longrightarrow} \mathcal{A} u t\left(\widehat{\mathcal{L}}_{S, \theta}^{c}\left(|\mathcal{L}|_{p}^{\wedge}\right)\right) \stackrel{c_{\xi}}{\longrightarrow} \mathcal{A} u t(\mathcal{L})
$$

whose composite will later be seen to be homotopic to the inclusion. The functor $R$ is easily defined: it sends an object $\mathcal{L} \stackrel{\psi}{\simeq} \mathcal{L}$ to the homotopy equivalence $|\mathcal{L}|_{p}^{\wedge} \stackrel{|\psi|_{p}^{\wedge}}{\longrightarrow}|\mathcal{L}|_{p}^{\wedge}$, and sends a natural isomorphism of functors to its realization as a homotopy between the induced maps.

On objects, $L$ sends a self homotopy equivalence $|\mathcal{L}|_{p}^{\wedge} \stackrel{f}{\longrightarrow}|\mathcal{L}|_{p}^{\wedge}$ to the functor $\widehat{\mathcal{L}}_{S, \theta}^{c}(f)$ induced by composition with $f$. To see that $\widehat{\mathcal{L}}_{S, \theta}^{c}(f)$ is a functor, note that for any object $(P, \alpha)$ in $\widehat{\mathcal{L}}_{S, \theta}^{c}\left(|\mathcal{L}|_{p}^{\wedge}\right),(P, f \circ \alpha)$ is also an object by Theorem $4.4(\mathrm{a})$ : $f \circ \alpha \simeq \theta \circ B \varphi$ for some $\varphi \in \operatorname{Hom}(P, S)$, and $\varphi$ must be injective since otherwise $\alpha$ would factor through $B(P / Q)$ for some $1 \neq Q \triangleleft P$. If $F:|\mathcal{L}|_{p}^{\wedge} \times I \longrightarrow|\mathcal{L}|_{p}^{\wedge}$ is a homotopy representing a morphism in $\mathcal{A} u t\left(|\mathcal{L}|_{p}^{\wedge}\right)$ from $f$ to $f^{\prime}$, then $L(F)$ is defined to be the natural isomorphism of functors which sends an object $(P, \alpha)$ to 
the morphism $\left(\operatorname{Id}_{P},[F \circ(\alpha \times I)]\right)$. (Note that this only depends on the homotopy class of $F$, as a path in $\operatorname{Aut}\left(|\mathcal{L}|_{p}^{\wedge}\right)$ from $f$ to $f^{\prime}$.) One easily checks that $L$ preserves compositions of homotopies and of homotopy equivalences, and is thus a a well defined functor of monoidal categories.

Since $\xi_{\mathcal{L}}: \mathcal{L} \longrightarrow \widehat{\mathcal{L}}_{S, \theta}^{c}\left(|\mathcal{L}|_{p}^{\wedge}\right)$ is an inclusion and an equivalence of categories (Proposition 7.3), it has a left inverse $\xi^{*}$, defined by sending any object $(P, \alpha)$ in $\widehat{\mathcal{L}}_{S, \theta}^{c}\left(|\mathcal{L}|_{p}^{\wedge}\right)$ not in the image of $\xi_{\mathcal{L}}$ to some $Q \leq S$ such that $\left(Q, i_{Q}\right)=\xi_{\mathcal{L}}(Q)$ is isomorphic to $(P, \alpha)$ in $\widehat{\mathcal{L}}_{S, \theta}^{c}\left(|\mathcal{L}|_{p}^{\wedge}\right)$. Define

$$
\mathcal{A} u t\left(\widehat{\mathcal{L}}_{S, \theta}^{c}\left(|\mathcal{L}|_{p}^{\wedge}\right)\right) \underset{\simeq}{\stackrel{c_{\xi}}{\longrightarrow}} \mathcal{A} u t(\mathcal{L})
$$

by setting $c_{\xi}(\psi)=\xi^{*} \circ \psi \circ \xi_{\mathcal{L}}$ for any self equivalence $\psi$ of $\widehat{\mathcal{L}}_{S, \theta}^{c}\left(|\mathcal{L}|_{p}^{\wedge}\right)$, and similarly for morphisms.

Lemma 8.3. The composite of the functors

$$
\mathcal{A} u t_{\text {typ }}(\mathcal{L}) \stackrel{R}{\longrightarrow} \mathcal{A} u t\left(|\mathcal{L}|_{p}^{\wedge}\right) \stackrel{L}{\longrightarrow} \mathcal{A} u t\left(\widehat{\mathcal{L}}_{S, \theta}^{c}\left(|\mathcal{L}|_{p}^{\wedge}\right)\right) \underset{\simeq}{\stackrel{c_{\xi}}{\longrightarrow}} \mathcal{A} u t(\mathcal{L})
$$

induces the inclusion $\pi_{0}\left(\left|\mathcal{A} u t_{\text {typ }}(\mathcal{L})\right|\right) \longrightarrow \pi_{0}(|\mathcal{A} u t(\mathcal{L})|)$, and the identity on

$$
\pi_{1}\left(\left|\mathcal{A} u t_{\text {typ }}(\mathcal{L})\right|, \operatorname{Id}\right)=\pi_{1}(|\mathcal{A} u t(\mathcal{L})|, \operatorname{Id}) \cong \underset{\mathcal{O}^{c}(\mathcal{F})}{\lim ^{0}(\mathcal{Z})}
$$

Proof. Step 1: Fix an isotypical equivalence $\psi: \mathcal{L} \longrightarrow \mathcal{L}$, and consider the following diagram:

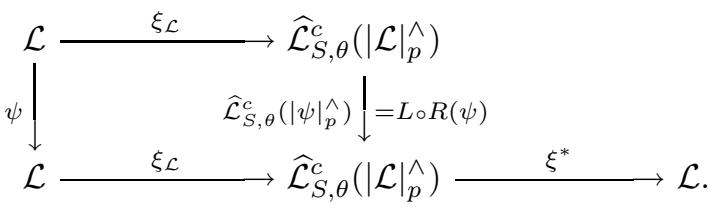

Here, $\xi^{*}$ is the left inverse of $\xi_{\mathcal{L}}$ used to define $c_{\xi}$. In particular, $c_{\xi} \circ L \circ R(\psi)=$ $\xi^{*} \circ \widehat{\mathcal{L}}_{S, \theta}^{c}\left(|\psi|_{p}^{\wedge}\right) \circ \xi_{\mathcal{L}}$, and proving the first part of the proposition means showing that the square commutes up to a natural isomorphism of functors.

Recall that $\xi_{\mathcal{L}}(P)=\left(P,\left|\theta_{P}\right|_{p}^{\wedge}\right)$ for all $P \leq S$, and that $\xi_{\mathcal{L}}(\alpha)=\left([\alpha],\left[\left|\eta_{\alpha}\right|_{p}^{\wedge}\right]\right)$ for each morphism $\alpha \in \operatorname{Mor}_{\mathcal{L}}(P, Q)$. Here, $\theta_{P} \stackrel{\eta_{\alpha}}{\longrightarrow} \theta_{Q} \circ \mathcal{B}([\alpha])$ is the natural transformation of functors $\mathcal{B}(P) \longrightarrow \mathcal{L}$ which sends the object $o_{P}$ to the morphism $\alpha$.

We write for short $P^{\prime}=\psi(P)$ for any object $P$ in $\mathcal{L}$, and $\alpha^{\prime}=\psi(\alpha)$ for any morphism $\alpha$. Define a natural transformation

$$
W(\psi): \widehat{\mathcal{L}}_{S, \theta}^{c}\left(|\psi|_{p}^{\wedge}\right) \circ \xi_{\mathcal{L}} \longrightarrow \xi_{\mathcal{L}} \circ \psi
$$

by sending an object $P$ in $\mathcal{L}$ to the morphism

$$
\left(\psi_{P}, C_{P}\right) \in \operatorname{Mor}_{\widehat{\mathcal{L}}_{S, \theta}^{c}(|\mathcal{L}|)}\left(\left(P,\left|\psi \circ \theta_{P}\right|_{p}^{\wedge}\right),\left(P^{\prime},\left|\theta_{P^{\prime}}\right|_{p}^{\wedge}\right)\right)
$$

Here $C_{P}$ denotes the constant homotopy. To see that $W(\psi)$ is a natural isomorphism of functors, note first that its source and target are correct:

$$
\widehat{\mathcal{L}}_{S, \theta}^{c}\left(|\psi|_{p}^{\wedge}\right)\left(\xi_{\mathcal{L}}(P)\right)=\left(P,\left|\psi \circ \theta_{P}\right|_{p}^{\wedge}\right) \quad \text { and } \quad \xi_{\mathcal{L}}(\psi(P))=\left(P^{\prime},\left|\theta_{P^{\prime}}\right|_{p}^{\wedge}\right)
$$

by definition. Also, $\left(\psi_{P}, C_{P}\right)$ is a morphism between these objects, since

$$
\left|\psi \circ \theta_{P}\right|=\left|\theta_{P^{\prime}} \circ \mathcal{B}\left(\psi_{P}\right)\right|=\left|\theta_{P^{\prime}}\right| \circ B \psi_{P}
$$


by definition of $\psi_{P}$. To show that $W(\psi)$ is natural, we must check, for each morphism $P \stackrel{\alpha}{\longrightarrow} Q$ in $\mathcal{L}$, that the following square commutes:



in $\widehat{\mathcal{L}}_{S, \theta}^{c}\left(|\mathcal{L}|_{p}^{\wedge}\right)$. By Lemma 8.2, $\left(P \mapsto \psi_{P}\right)$ is a natural isomorphism of functors $F \stackrel{\cong}{\longrightarrow} F \circ \psi$ (where $F: \mathcal{L} \longrightarrow \mathrm{Gr}$ is the forgetful functor), and thus $\left[\alpha^{\prime}\right] \circ \psi_{P}=$ $\psi_{Q} \circ[\alpha]$. So it remains to show that $\left|\eta_{\alpha^{\prime}}\right|_{p}^{\wedge} \circ\left(B \psi_{P} \times I\right)=\left|\psi \circ \eta_{\alpha}\right|_{p}^{\wedge}$, and this follows since both are induced by the natural isomorphism $\left(o_{P} \mapsto \alpha^{\prime}\right)$ of functors $\mathcal{B}(P) \longrightarrow \mathcal{L}$.

Step 2: We next prove that the groups in $(1)$ are isomorphic. Since $\mathcal{A} u t(\mathcal{L})$ is a groupoid,

$$
\pi_{1}\left(\left|\mathcal{A} u t_{\text {typ }}(\mathcal{L})\right|, \mathrm{Id}\right)=\pi_{1}(|\mathcal{A} u t(\mathcal{L})|, \mathrm{Id}) \cong \operatorname{Aut}_{\mathcal{A} u t(\mathcal{L})}(\mathrm{Id}) .
$$

A natural isomorphism of functors Id $\stackrel{\Psi}{\longrightarrow}$ Id is given by morphisms $\Psi_{P} \in \operatorname{Aut}_{\mathcal{L}}(P)$ for all $\mathcal{F}$-centric $P \leq S$, such that $\alpha \circ \Psi_{P}=\Phi_{Q} \circ \alpha$ for each morphism $\alpha \in$ $\operatorname{Mor}_{\mathcal{L}}(P, Q)$. In particular, for each $P, \Psi_{P}$ lies in the center of $\operatorname{Aut}_{\mathcal{L}}(P)$, so $\pi_{P, P}\left(\Psi_{P}\right)=\operatorname{Id}_{P} \in \operatorname{Aut}(P)$ by condition (C) (and Lemma 1.10(b)), and thus $\Psi_{P}=\widehat{g_{P}}$ for some $g_{P} \in Z(P)$. The naturality of $\Psi$ now shows that the elements $g_{P}$ combine to define

$$
\left(g_{P}\right)_{P \in \mathrm{Ob}(\mathcal{L})} \in{\underset{\mathcal{O}^{c}(\mathcal{F})}{\lim ^{0}}(\mathcal{Z})}
$$

The converse is clear - any such collection of elements $g_{P} \in Z(P)$ defines a natural isomorphism of functors - and this proves (1).

Step 3: It remains to show that $c_{\xi} \circ L \circ R$ induces the identity on $\pi_{1}(|\mathcal{A} u t(\mathcal{L})|)$. Fix an element in this group, represented by a natural isomorphism Id $\stackrel{\Psi}{\longrightarrow}$ Id of functors, and write $\Psi_{P}=\widehat{g_{P}}$, where $g_{P} \in Z(P)$ for each $P$. Let [1] denote the category with two objects 0,1 and one nonidentity morphism $0 \stackrel{\iota}{\longrightarrow}$. Then $R(\Psi)$ is the homotopy on $|\mathcal{L}|_{p}^{\wedge}$ induced by the functor

$$
\Psi_{*}: \mathcal{L} \times[1] \longrightarrow \mathcal{L},
$$

defined by setting $\Psi(P, t)=P(t=0,1), \Psi\left(\alpha, \operatorname{Id}_{t}\right)=\alpha$, and $\Psi\left(\operatorname{Id}_{P}, \iota\right)=\Psi_{P}$. Hence $L(R(\Psi))$, as a natural isomorphism of functors from $\widehat{\mathcal{L}}_{S, \theta}^{c}\left(|\mathcal{L}|_{p}^{\wedge}\right)$ to itself, sends each object $\left(P,\left|\theta_{P}\right|_{p}^{\wedge}\right)$ to the morphism

$$
\left(\operatorname{Id}_{P},\left|\Psi_{*} \circ\left(\theta_{P} \times \operatorname{Id}_{[1]}\right)\right|_{p}^{\wedge}\right)=\left(\operatorname{Id}_{P},\left|\eta_{g_{P}}\right|_{p}^{\wedge}\right)=\xi_{\mathcal{L}}\left(\widehat{g_{P}}\right)=\xi_{\mathcal{L}}\left(\Psi_{P}\right) .
$$

Since $\xi^{*} \circ \xi_{\mathcal{L}}=\operatorname{Id}_{\mathcal{L}}$, this shows that $c_{\xi} \circ L \circ R(\Psi)=\Psi$.

It remains to show that $|L|$ induces a monomorphism on homotopy groups.

Lemma 8.4. The map

$$
|L|:\left|\mathcal{A} u t\left(|\mathcal{L}|_{p}^{\wedge}\right)\right| \longrightarrow\left|\mathcal{A} u t\left(\widehat{\mathcal{L}}_{S, \theta}^{c}\left(|\mathcal{L}|_{p}^{\wedge}\right)\right)\right|
$$

induces monomorphisms on $\pi_{0}$ and on $\pi_{1}$. Also, $\pi_{n}\left(\operatorname{Aut}\left(|\mathcal{L}|_{p}^{\wedge}\right)\right)=0$ for all $n>1$. 
Proof. The proof is based on the decomposition

$$
\operatorname{pr}: \underset{\mathcal{O}^{c}(\mathcal{F})}{\operatorname{hocolim}}(\widetilde{B}) \stackrel{\simeq}{\longrightarrow}|\mathcal{L}|
$$

of Proposition 2.2, where $\widetilde{B}: \mathcal{O}^{c}(\mathcal{F}) \longrightarrow$ Top is a lifting of the homotopy functor $P \mapsto B P$. In the following constructions, we regard $\underline{\operatorname{hocolim}}(\widetilde{B})$ as the union of skeleta:

$$
\underset{\mathcal{O}^{c}(\mathcal{F})}{\operatorname{\operatorname {hocolim}^{(n)}}}(\widetilde{B})=\left(\coprod_{i=0}^{n} \coprod_{P_{0} \rightarrow \cdots \rightarrow P_{n}} \widetilde{B}\left(P_{0}\right) \times D^{i}\right) / \sim,
$$

where we divide out by the usual face and degeneracy relations.

To simplify the notation, we write $i_{P}=\left|\theta_{P}\right|_{p}^{\wedge}: B P \longrightarrow|\mathcal{L}|_{p}^{\wedge}$ for each subgroup $P \leq S$. The obstructions to extending a map

$$
\left.\left(\underset{\mathcal{O}^{c}(\mathcal{F})}{\operatorname{hocolim}}(\widetilde{B}) \times S^{k-1}\right) \cup \underset{\mathcal{O}^{c}(\mathcal{F})}{\left(\operatorname{hocolim}^{(n-1)}\right.}(\widetilde{B}) \times D^{k}\right) \longrightarrow|\mathcal{L}|_{p}^{\wedge}
$$

to $\left.\underline{\operatorname{hocolim}}^{(n)}(\widetilde{B}) \times D^{k}\right)$ lie in the groups

$$
\pi_{n+k}\left(\operatorname{Map}\left(B P,|\mathcal{L}|_{p}^{\wedge}\right)_{B i_{P}}\right) \cong\left\{\begin{array}{ll}
Z(P) & \text { if } n+k=1 \\
0 & \text { if } n+k>1
\end{array},\right.
$$

(see Theorem 4.4(c)) for $\mathcal{F}$-centric subgroups $P \leq S$.

We prove the injectivity on $\pi_{0}$ in Step 1 . The injectivity on $\pi_{1}$, together with the vanishing of higher homotopy groups, is shown in Step 2.

Step 1: Fix a homotopy equivalence $f:|\mathcal{L}|_{p}^{\wedge} \longrightarrow|\mathcal{L}|_{p}^{\wedge}$ such that $[f] \in$ $\operatorname{Ker}\left(\pi_{0}(L)\right)$, and let $\Psi: \widehat{\mathcal{L}}_{S, \theta}^{c}(f) \longrightarrow$ Id be a natural isomorphism. For each $\mathcal{F}$ centric $P \leq S$, write $\Psi\left(P, i_{P}\right)=\left(\sigma_{P},\left[\omega_{P}\right]\right)$, an isomorphism from $\left(P, f \circ i_{P}\right)$ to $\left(P, i_{P}\right)$. Thus, $\sigma_{P}$ is an automorphism of $P, \omega_{P}$ is a path in $\operatorname{Map}\left(B P,|\mathcal{L}|_{p}^{\wedge}\right)$ from $f \circ i_{P}$ to $i_{P} \circ B \sigma_{P}$, and $\left[\omega_{P}\right]$ is its homotopy class relative to its endpoints.

We first show, for each $P$, that $\sigma_{P}=\operatorname{Id}_{P}$. Set $\sigma=\sigma_{P}$ and $\omega=\omega_{P}$ for short. Fix $g \in P$, and consider the following two squares of morphisms in $\widehat{\mathcal{L}}_{S, \theta}^{c}\left(|\mathcal{L}|_{p}^{\wedge}\right)$ :

$$
\begin{aligned}
& \left(P, f \circ i_{P}\right) \stackrel{(\sigma, \omega)}{\longrightarrow}\left(P, i_{P}\right) \quad\left(P, f \circ i_{P}\right) \stackrel{(\sigma, \omega)}{\longrightarrow}\left(P, i_{P}\right) \\
& \left(c_{g}, f \circ i_{P} \circ \eta_{g}^{P}\right) \downarrow \quad\left(c_{g}, i_{P} \circ \eta_{g}^{P}\right) \downarrow \quad \text { and } \quad{\left(c_{g}, f \circ i_{P} \circ \eta_{g}^{P}\right)} \downarrow\left(c_{\sigma(g)}, i_{P} \circ \eta_{\sigma(g)}^{P}\right) \\
& \left(P, f \circ i_{P}\right) \stackrel{(\sigma, \omega)}{\longrightarrow}\left(P, i_{P}\right) \quad\left(P, f \circ i_{P}\right) \stackrel{(\sigma, \omega)}{\longrightarrow}\left(P, i_{P}\right) .
\end{aligned}
$$

Here, $\eta_{g}^{P}$ denotes the path in $\operatorname{Map}(B P, B P)$ from Id to $B c_{g}$ induced by the natural transformation of functors $\mathcal{B}(P) \longrightarrow \mathcal{B}(P)$ which sends $o_{P}$ to $\check{g}$. The first square commutes by the naturality of $\Psi$ with respect to $\left(P, i_{P}\right) \stackrel{\left(c_{g}, i_{P} \circ \eta_{g}^{P}\right)}{\longrightarrow}\left(P, i_{P}\right)$. The second commutes since $\sigma \circ c_{g}=c_{\sigma(g)} \circ \sigma$, and since the square

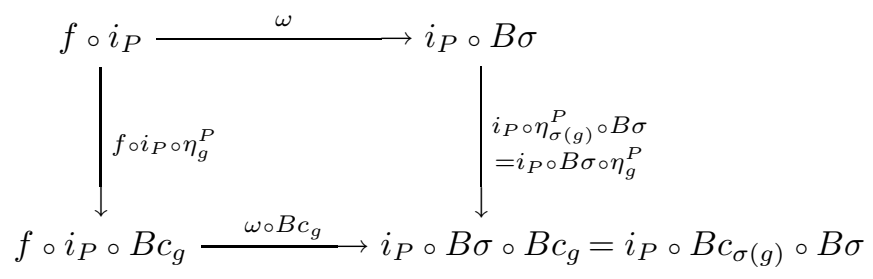


of paths in $\operatorname{Map}\left(B P,|\mathcal{L}|_{p}^{\wedge}\right)$ commutes via the homotopy

$$
H: I \times I \longrightarrow \operatorname{Map}\left(B P,|\mathcal{L}|_{p}^{\wedge}\right) \quad \text { defined by } \quad H(s, t)=\omega(s) \circ \eta_{g}^{P}(t) .
$$

Since all of the maps in (2) are isomorphisms, it now follows that $c_{g}=c_{\sigma(g)}$ (so $\left.g^{-1} \sigma(g) \in Z(P)\right)$, and that the loop $i_{P} \circ \eta_{g^{-1} \sigma(g)}^{P}$ in $\operatorname{Map}\left(B P,|\mathcal{L}|_{p}^{\wedge}\right)_{i_{P}}$ is null homotopic. Hence $g=\sigma(g)$ by (1), and $\sigma=\sigma_{P}=$ Id.

Now consider the composite

$$
\underset{\mathcal{O}^{c}(\mathcal{F})}{\operatorname{hocolim}}(\widetilde{B}) \stackrel{\kappa}{\longrightarrow}|\mathcal{L}|_{p}^{\wedge} \stackrel{f}{\longrightarrow}|\mathcal{L}|_{p}^{\wedge},
$$

where $\kappa$ is the equivalence of Proposition 2.2 (after completion). Since hocolim ${ }^{(0)}(\widetilde{B})$ is the disjoint union of the $\widetilde{B}(P) \simeq B P$, and $\left.\kappa\right|_{B P}=i_{P}$, the $\omega_{P}: f \circ i_{P} \simeq i_{P}$ define a homotopy on $\underline{\operatorname{hocolim}}^{(0)}(\widetilde{B})$ between $f \circ \kappa$ and $\kappa$. The naturality of the $\omega_{P}$ implies that this can be extended to a homotopy on the 1-skeleton $\underline{\operatorname{hocolim}}^{(1)}(\widetilde{B})$, and hence by (1) to all of $\underset{\operatorname{hocolim}}{\longrightarrow}(\widetilde{B})$. Since $\kappa$ is a mod $p$ homology equivalence, this shows that $f \simeq \operatorname{Id}$.

Step 2: An element $[F] \in \operatorname{Ker}\left(\pi_{1}(|L|)\right)$ is the pointed homotopy class of a map

$$
F: S^{1} \times|\mathcal{L}|_{p}^{\wedge} \longrightarrow|\mathcal{L}|_{p}^{\wedge}
$$

such that for all $\mathcal{F}$-centric $P \leq S,\left.F\right|_{S^{1} \times B P}$ extends to a map on $D^{2} \times B P$. The composite

$$
S^{1} \times \underset{\mathcal{O}^{c}(\mathcal{F})}{\operatorname{hocolim}}(\widetilde{B}) \stackrel{S^{1} \times \mathrm{pr}}{\longrightarrow} S^{1} \times|\mathcal{L}|_{p}^{\wedge} \stackrel{F}{\longrightarrow}|\mathcal{L}|_{p}^{\wedge}
$$

can thus be extended to $D^{2} \times \operatorname{hocolim}^{(0)}(\widetilde{B})$, and hence to all of $D^{2} \times \underset{\text { hocolim}}{\longrightarrow}(\widetilde{B})$ by (1). Since pr is a mod $p$ homology equivalence, it now follows that $[\overrightarrow{F]=1}$, and thus that $\pi_{1}(|T|)$ is injective.

The proof that $\pi_{n}\left(\operatorname{Aut}\left(|\mathcal{L}|_{p}^{\wedge}\right)\right)=0$ for all $n>1$ also follows easily from the homotopy colimit decomposition of $|\mathcal{L}|_{p}^{\wedge}$, together with (1). (See also BL, Proposition 3.6].)

We must show that $\operatorname{Aut}\left(|\mathcal{L}|_{p}^{\wedge}\right)$ and $|\mathcal{A} u t(\mathcal{L})|$ are homotopy equivalent, and moreover equivalent as monoids. There is no obvious way to construct a map between these two spaces which is both a homotopy equivalence and a morphism of monoids, so instead we connect them with a sequence of maps going in alternating directions.

Let $S$. Aut $\left(|\mathcal{L}|_{p}^{\wedge}\right)$ denote the singular simplicial set of $\operatorname{Aut}\left(|\mathcal{L}|_{p}^{\wedge}\right)$; an $n$-simplex is thus a homotopy equivalence $\Delta^{n} \times|\mathcal{L}|_{p}^{\wedge} \longrightarrow|\mathcal{L}|_{p}^{\wedge}$. Let

$$
\operatorname{Aut}\left(|\mathcal{L}|_{p}^{\wedge}\right) \stackrel{\text { ev }}{\longleftarrow}\left|S \cdot \operatorname{Aut}\left(|\mathcal{L}|_{p}^{\wedge}\right)\right| \stackrel{\sigma}{\longrightarrow}\left|\mathcal{A} u t\left(|\mathcal{L}|_{p}^{\wedge}\right)\right|
$$

denote the obvious maps: the first is the evaluation map $\left|S_{\mathbf{.}} X\right| \longrightarrow X$ defined for any space $X$, and the second is the map $|S . X| \longrightarrow|\pi(X)|$ which sends each simplex to its homotopy class. Both are morphisms of monoids.

Proof of Theorem 8.1. The following maps are morphisms of topological monoids:

$$
\operatorname{Aut}\left(|\mathcal{L}|_{p}^{\wedge}\right) \stackrel{\text { ev }}{\longleftarrow}\left|S . \operatorname{Aut}\left(|\mathcal{L}|_{p}^{\wedge}\right)\right| \stackrel{\sigma}{\longrightarrow}\left|\mathcal{A} u t\left(|\mathcal{L}|_{p}^{\wedge}\right)\right| \stackrel{|R|}{\longleftarrow}\left|\mathcal{A} u t_{\text {typ }}(\mathcal{L})\right| .
$$

The first is a (weak) homotopy equivalence by definition, and the second is a homotopy equivalence since $\operatorname{Aut}\left(|\mathcal{L}|_{p}^{\wedge}\right)$ is aspherical (Lemma 8.4 or $[\overline{\mathrm{BL}}$ ). Finally, $|R|$ 
induces isomorphisms on all homotopy groups by Lemmas 8.3 and 8.4 The classifying spaces $B \operatorname{Aut}\left(|\mathcal{L}|_{p}^{\wedge}\right)$ and $B\left|\mathcal{A} u t_{\text {typ }}(\mathcal{L})\right|$ are thus homotopy equivalent, since a morphism of monoids which is a homotopy equivalence induces a homotopy equivalence between the classifying spaces (cf. [GJ Proposition IV.1.7]).

In particular, this shows that $\operatorname{Out}\left(|\mathcal{L}|_{p}^{\wedge}\right) \cong \operatorname{Out}_{\text {typ }}(\mathcal{L})$. The isomorphism

$$
\pi_{1}\left(\operatorname{Aut}\left(|\mathcal{L}|_{p}^{\wedge}\right)\right) \cong{\underset{\mathcal{O}^{c}(\mathcal{F})}{\lim ^{0}}(\mathcal{Z})}
$$

was shown in Lemma 8.3

\section{EXAmples}

We now look at some explicit examples of $p$-local finite groups, and in particular of $p$-local finite groups which are not induced from actual finite groups. The main problem is to find new ways of constructing saturated fusion systems. One general procedure for constructing such systems is given here in Proposition 9.1 and two concrete applications of this proposition are given in Examples 9.3 and 9.4 To show that some of these examples are "exotic" $p$-local finite groups, we first prove a result (Lemma 9.2) which shows under certain hypotheses that if $\mathcal{F}$ is the fusion system of a finite group, then it is the fusion system of an almost simple group; and afterwards list all finite simple groups (Proposition 9.5) which have a certain type of Sylow subgroup. The proof of Proposition 9.5 is based on the classification theorem for finite simple groups, and for lack of space we only sketch its proof and give the necessary references.

In a later paper, we will construct more examples, including some which are closely related to certain exotic $p$-compact groups, and to spaces constructed by Benson [Be, $\S 8]$ and Broto and Møller [BrM] by taking homotopy fixed point sets of Adams operations on certain $p$-compact groups. In particular, we will construct $p$-local finite groups at the prime two, whose fusion systems, over Sylow subgroups of $\operatorname{Spin}(7, q)$, were shown by Solomon [Sol] not to be fusion systems of finite groups.

We focus attention here on a particularly simple class of saturated fusion systems: those systems $\mathcal{F}$ over a $p$-group $S$ for an odd prime $p$, with the property that $S$ is nonabelian and contains a homocyclic subgroup (a product of cyclic subgroups of the same order) of index $p$ and rank $r$, where $3 \leq r \leq p$. In particular, Corollary 3.5 applies in all of these cases to show that $\mathcal{F}$ has a unique associated centric linking system.

We first need some general definitions. Let $\mathcal{F}$ and $\mathcal{F}^{\prime}$ be two fusion systems over a $p$-group $S$. By the fusion system generated by $\mathcal{F}$ and $\mathcal{F}^{\prime}$, we mean the smallest fusion system which contains them, i.e., the fusion system $\left\langle\mathcal{F}, \mathcal{F}^{\prime}\right\rangle$ such that for all $P, P^{\prime} \leq S$ the morphism set $\operatorname{Hom}_{\left\langle\mathcal{F}, \mathcal{F}^{\prime}\right\rangle}\left(P, P^{\prime}\right)$ is the set of composites

$$
P=P_{0} \stackrel{\varphi_{1}}{\longrightarrow} P_{1} \stackrel{\varphi_{2}}{\longrightarrow} P_{2} \longrightarrow \cdots \longrightarrow P_{k-2} \stackrel{\varphi_{k-1}}{\longrightarrow} P_{k-1} \stackrel{\varphi_{k}}{\longrightarrow} P_{k}=P^{\prime}
$$

such that, for each $i, \varphi_{i}$ lies in $\operatorname{Hom}_{\mathcal{F}}\left(P_{i-1}, P_{i}\right)$ or in $\operatorname{Hom}_{\mathcal{F}^{\prime}}\left(P_{i-1}, P_{i}\right)$. The fusion system generated by two saturated fusion systems need not, of course, be saturated.

More generally, if $\mathcal{F}_{0}$ is a fusion system over a $p$-group $S$, and for each $i=$ $1, \ldots, m$ we are given subgroups $Q_{i} \leq S$ and fusion systems $\mathcal{F}_{i}$ over $Q_{i}$, then we let $\mathcal{F} \stackrel{\text { def }}{=}\left\langle\mathcal{F}_{0} ; \mathcal{F}_{1}, \ldots, \mathcal{F}_{m}\right\rangle$ be the fusion system over $S$ defined as follows. For each pair of subgroups $P, P^{\prime} \leq S, \operatorname{Hom}_{\mathcal{F}}\left(P, P^{\prime}\right)$ is the set of composites

$$
P=P_{0} \stackrel{\varphi_{1}}{\longrightarrow} P_{1} \stackrel{\varphi_{2}}{\longrightarrow} P_{2} \longrightarrow \cdots \longrightarrow P_{k-2} \stackrel{\varphi_{k-1}}{\longrightarrow} P_{k-1} \stackrel{\varphi_{k}}{\longrightarrow} P_{k}=P^{\prime},
$$


where for each $i$, either $\varphi_{i} \in \operatorname{Hom}_{\mathcal{F}_{0}}\left(P_{i-1}, P_{i}\right)$, or $P_{i-1}, P_{i} \leq Q_{j}$ for some $j$ and $\varphi_{i} \in \operatorname{Hom}_{\mathcal{F}_{j}}\left(P_{i-1}, P_{i}\right)$.

If $Q$ is a $p$-group and $\operatorname{Inn}(Q) \leq \Delta \leq \operatorname{Aut}(Q)$, then $\mathcal{F}_{Q}(\Delta)$ will denote the fusion system over $Q$ whose morphisms are the restrictions of elements of $\Delta$ to subgroups of $Q$.

We can now formulate the main proposition used here to construct examples of saturated fusion systems. Throughout its proof, as well as the rest of the section, we write $C_{n}$ to denote a multiplicative cyclic group of order $n$.

Proposition 9.1. Fix an odd prime $p$, a finite group $G$, and a normal abelian $p$ subgroup $A \triangleleft G$. Fix a Sylow p-subgroup $S \leq G$, and set $Z=Z(S)$. Assume that $S$ is nonabelian and $[A: Z] \geq p^{2}$. Thus, $A \triangleleft S$ and $Z=A^{S / A}$. Let $\mathcal{Q}=\left\{Q_{1}, \ldots, Q_{m}\right\}$ be a set of subgroups of $S$ such that for each $Q \in \mathcal{Q}, Q \geq Z$, and either $Q$ or $Q / Z(Q)$ is elementary abelian of rank two. Fix, for each $Q \in \mathcal{Q}$, a subgroup $\Delta_{Q} \leq \operatorname{Aut}(Q)$ containing $\operatorname{Inn}(Q)$. Assume the following hold:

(a) $[S: A]=p$ and $Z$ is cyclic.

(b) For $Q_{i}, Q_{j} \in \mathcal{Q}$ with $i \neq j$, no element of $Q_{i} \backslash A$ is $G$-conjugate to any element of $Q_{j} \backslash A$.

(c) For each $Q \in \mathcal{Q},\left\{\varphi \in \Delta_{Q} \mid \varphi(Q \cap A)=Q \cap A\right\}=\operatorname{Aut}_{G}(Q)$.

Then the fusion system $\mathcal{F} \stackrel{\text { def }}{=}\left\langle\mathcal{F}_{S}(G) ; \mathcal{F}_{Q_{1}}\left(\Delta_{1}\right), \ldots, \mathcal{F}_{Q_{m}}\left(\Delta_{m}\right)\right\rangle$ is saturated (where $\left.\Delta_{i}=\Delta_{Q_{i}}\right)$. Furthermore, $\operatorname{Aut}_{\mathcal{F}}(A)=\operatorname{Aut}_{G}(A) \cong G / C_{G}(A)$.

Proof. From the assumptions on $Q \in \mathcal{Q}\left(Q \cong C_{p}^{2}\right.$ or $\left.Q / Z(Q) \cong C_{p}^{2}\right)$ and the assumption $[A: Z] \geq p^{2}$, we see that neither $A$ nor $S$ can be in $\mathcal{Q}$. Thus no $Q \in \mathcal{Q}$ contains $A$, and this proves that $\operatorname{Aut}_{\mathcal{F}}(A)=\operatorname{Aut}_{G}(A)$.

In addition to points $(\mathrm{a}-\mathrm{c})$ above, we can assume that

(d) for each $Q \in \mathcal{Q}, Q \not \leq A$, and there is some $\varphi \in \Delta_{Q}$ such that $\varphi(Q \cap A) \not \leq A$.

Otherwise, by (c), all morphisms in $\mathcal{F}_{Q}\left(\Delta_{Q}\right)$ are also in $\mathcal{F}_{S}(G)$, and hence $\mathcal{F}_{Q}\left(\Delta_{Q}\right)$ contributes nothing new to $\mathcal{F}$.

Let $V \leq A$ be the maximal elementary abelian subgroup. Since $Z=A^{S / A}$ is cyclic, $\left|V^{\bar{S} / A}\right|=p$, and $V$ must be indecomposable as an $\mathbb{F}_{p}[S / A]$-module since otherwise each summand would have nontrivial fixed submodule. Also, if $x$ is a generator of $S / A$, then

$$
\mathbb{F}_{p}[S / A] \cong \mathbb{F}_{p}[x] /\left(x^{p}-1\right)=\mathbb{F}_{p}[x] /(x-1)^{p} .
$$

Hence by the classification theorem for finitely generated modules over a principal ideal domain, $V \cong \mathbb{F}_{p}[x] /(x-1)^{n}$ (as an $S / A$-module) for some $n=\operatorname{dim}_{\mathbb{F}_{p}}(V) \leq p$, and its only submodules are the ideals generated by $(x-1)^{i}$ for $0 \leq i \leq n$. In other words, for each $0 \leq r \leq n$, there is a unique subgroup $V_{r}=(x-1)^{n-r} \cdot V \leq V$ of rank $r$ which is normal in $S$.

Step 1: We claim the following statements hold:

(1) If $P \leq S$ and $P \not \leq A$, then $P \cap V=V_{r}$ for some $r, C_{S}(P) \geq Z$, and

$$
\left[C_{S}(P): Z\right]= \begin{cases}1 & \text { if } P \text { is nonabelian } \\ p & \text { if } P \text { is abelian. }\end{cases}
$$


If $P$ is abelian, then $P \cap A \leq Z$, and

$$
N_{S}(P)= \begin{cases}P Z & \text { if } P \cap A=1, \\ P Z V_{2} & \text { if } P \cap A \neq 1 .\end{cases}
$$

(2) For each $Q \in \mathcal{Q}$, either $Q \cong C_{p}^{2}$ and $Q \cap A=Z$ has order $p$, or $Q$ is nonabelian, $Z(Q)=Z$ has index $p^{2}$ in $Q$, and $Q \cap A=Z V_{2}$.

(3) For each $Q \in \mathcal{Q}$, let $\mathcal{S}_{Q}$ be the set of all subgroups of $Q$ of index $p$ if $Q$ is abelian, or the set of subgroups of $Q$ of index $p$ which contain $Z(Q)=Z$ if $Q$ is nonabelian. Then $\left|\mathcal{S}_{Q}\right|=p+1$, and the elements of $\mathcal{S}_{Q}$ are permuted transitively by the group $\Delta_{Q}$. Furthermore, for each $P \in \mathcal{S}_{Q}$, there is $\psi \in \Delta_{Q}$ such that $\left.\psi\right|_{P}=\operatorname{Id}_{P}$, and such that $\psi$ permutes transitively the other subgroups in $\mathcal{S}_{Q}$.

(4) If $Q \in \mathcal{Q}, P, P^{\prime} \leq Q$, and $\varphi \in \operatorname{Iso}_{\Delta_{Q}}\left(P, P^{\prime}\right)$ are such that $\varphi(P \cap A)=P^{\prime} \cap A$, then $\varphi \in \operatorname{Iso}_{G}\left(P, P^{\prime}\right)$.

(5) If $Q \in \mathcal{Q}, P, P^{\prime} \leq Q$, and $P, P^{\prime} \not \leq A$, then $\operatorname{Iso}_{G}\left(P, P^{\prime}\right) \subseteq \operatorname{Iso}_{\Delta_{Q}}\left(P, P^{\prime}\right)$.

(6) If $Q \in \mathcal{Q}$, and $P, P^{\prime} \leq Q$ are not $\mathcal{F}$-conjugate to any subgroup of $A$, then $\operatorname{Iso}_{\mathcal{F}}\left(P, P^{\prime}\right)=\operatorname{Iso}_{\Delta_{Q}}\left(P, P^{\prime}\right)$.

(7) If $\varphi \in \operatorname{Iso}_{\mathcal{F}}\left(P, P^{\prime}\right)$, where either $P, P^{\prime} \leq A$, or $\varphi(P \cap A)=P^{\prime} \cap A$ and $P, P^{\prime}$ are not $\mathcal{F}$-conjugate to any subgroup of $A$, then $\varphi \in \operatorname{Iso}_{G}\left(P, P^{\prime}\right)$.

(8) For each subgroup $P \leq S$ which is $\mathcal{F}$-conjugate to a subgroup of $A$, there is $\psi \in \operatorname{Hom}_{\mathcal{F}}\left(N_{S}(P), S\right)$ such that $\psi(P) \leq A$.

(9) If $P \leq S$ is fully centralized in $\mathcal{F}$, then either $P \leq A$, or $P$ is not $\mathcal{F}$-conjugate to any subgroup of $A$.

The most important points in the above list are (6), (7), and (8), which give the necessary information about morphisms in $\mathcal{F}$. Points (1), (3), and (9) will also be used in Step 2, while the others are only needed to prove later points in this list.

Proof of (1). Assume $P \leq S$ and $P \not \leq A$, and fix $x \in P \backslash A$. Thus, $x A$ generates $S / A$. Since $P \cap V$ and $P \cap A$ are normalized by $x$, they are $S / A$-invariant subgroups of $A$. In particular, $P \cap V=V_{r}$, where $r=\operatorname{rk}(P \cap V)$. Also, $A \cap C_{S}(P)=A^{\langle x\rangle}=Z$, so $Z$ has index 1 or $p$ in $C_{S}(P)$, and has index $p$ if and only if $x \in C_{S}(P)$, if and only if $P$ is abelian.

If $P$ is abelian, then

$$
P \cap A \leq A^{\langle x\rangle}=Z \quad \text { and } \quad C_{S}(x)=P \cdot A^{\langle x\rangle}=P Z .
$$

Clearly, $P Z \leq N_{S}(P)$. If $P \cap A=1$, then for any $g \in N_{S}(P),[x, g] \in[S, S] \cap P \leq$ $A \cap P=1$, and hence $g \in C_{S}(x)=P Z$. Thus, $N_{S}(P)=P Z$ in this case.

Now assume that $P \cap A \neq 1$. In particular, since $P \cap A \leq Z$ and $Z$ is cyclic, we have $P \cap A \geq V_{1}$. Also,

$$
\left[P, V_{2}\right]=\left[x, V_{2}\right]=(x-1) \cdot\left((x-1)^{n-2} \cdot V\right)=(x-1)^{n-1} \cdot V=V_{1},
$$

and so $V_{2} \leq N_{S}(P)$. Thus $P Z V_{2} \leq N_{S}(P)$, and it remains to prove the opposite inclusion. For any $g \in N_{S}(P)$,

$$
z \stackrel{\text { def }}{=}[x, g] \in[S, S] \cap P \leq A \cap P \leq Z=Z(S) .
$$


Thus $x g x^{-1}=z g$, so $x^{p} g x^{-p}=z^{p} g$, and $z^{p}=1$ since $x^{p} \in A \cap P \leq Z$. Since $z \in Z$ and has order at most $p, z \in V_{1}$, and hence there is $h \in V_{2}$ such that $[x, h]=z=[x, g]$. This implies that $\left[x, g h^{-1}\right]=1$, hence that $g h^{-1} \in C_{S}(x)=P Z$, and hence that $g \in P Z V_{2}$.

Proof of (2). Fix $Q \in \mathcal{Q}$. Then $Q \not \leq A$ by (d), and $Q \cap A \geq Z$ since $Q \geq Z$ by assumption. If $Q$ is abelian, then it is elementary abelian of rank 2 by assumption, and hence $Q \cap A=Z$ by (1). In particular, $|Q \cap A|=|Z|=p$, since $Z$ is both cyclic and elementary abelian.

Now assume $Q$ is nonabelian, and fix $x \in Q \backslash A$. Then $[x, Q] \neq 1$, so $Z(Q)=$ $(Q \cap A)^{\langle x\rangle} \leq Z$, and $Z(Q)=Z=Z(S)$ since $Q \geq Z$ by assumption. By (d), there is $\varphi \in \Delta_{Q}$ such that $\varphi(Q \cap A) \not \leq A$. Then $\varphi(Z)=Z$ (since $Z=Z(Q)$ ), and

$$
Z=A \cap \varphi(Z) \leq A \cap \varphi(Q \cap A) \leq Z,
$$

where the last inequality holds by $(1)$ since $\varphi(Q \cap A)$ is abelian and not contained in $A$. Thus, $A \cap \varphi(Q \cap A)=Z$. Also,

$$
Q \leq N_{S}(\varphi(Q \cap A))=\varphi(Q \cap A) \cdot V_{2} ;
$$

the equality holds by (1), and the inequality since $Q \cap A \triangleleft Q$ and automorphisms preserve normality. Since $Q$ strictly contains $\varphi(Q \cap A)$ and

$$
\left[\varphi(Q \cap A) \cdot V_{2}: \varphi(Q \cap A)\right]=\left[V_{2}: V_{1}\right]=p,
$$

this last inequality is an equality. Thus $Q \cap A=Z V_{2}$, and $[Q: Z]=[Q: Z(Q)]=p^{2}$ by assumption.

Proof of (3). Fix $Q \in \mathcal{Q}$. Set $Q_{0}=1$ if $Q$ is abelian, and $Q_{0}=Z(Q)=Z$ if $Q$ is nonabelian. In either case, $Q / Q_{0}$ is elementary abelian of rank 2 , and $\mathcal{S}_{Q}$ is the set of subgroups of index $p$ in $Q$ which contain $Q_{0}$. Thus $\left|\mathcal{S}_{Q}\right|=p+1$. Set

$$
\mathcal{S}_{Q}=\left\{P_{0}=Q \cap A, P_{1}, \ldots, P_{p}\right\} .
$$

Fix an element $x \in Q \backslash A$. By (2), either $Q \cap A=Z$ (if $Q_{0}=1$ ) or $Q \cap A=Z V_{2}$ (if $Q_{0}=Z$ ). In the first case choose $g \in V_{2} \backslash V_{1}$, and in the second case $g \in V_{3} \backslash V_{2}$. Since $\left[x, V_{r}\right]=V_{r-1}$ for all $r \geq 1$, we have $[Q, g]=\langle[x, g]\rangle \leq Q$ in both cases by (2), and so $g \in N_{S}(Q) \backslash Q$. (In either case, $g \notin Q$ by the description of $Q \cap A$.) Thus $\left.\left(c_{g}\right)\right|_{Q} \in \Delta_{Q}$, since $\operatorname{Aut}_{G}(Q) \leq \Delta_{Q}$ by (c). Moreover, $g$ centralizes $Q \cap A$ since $g \in A$. Also, for each $i=1, \ldots, p$, either

- $Q \cap A=Z, g \in V_{2} \backslash V_{1}, P_{i} \cap A=1$, and $[g, x] \in V_{1} \backslash 1$; or

- $Q \cap A=Z V_{2}, g \in V_{3} \backslash V_{2}, P_{i} \cap A=Z$, and $[g, x] \in V_{2} \backslash V_{1}$.

In all cases, $[g, x] \notin P_{i}$, so $g P_{i} g^{-1} \neq P_{i}$, and this shows that the subgroup generated by $c_{g}$ permutes the subgroups $P_{1}, \ldots, P_{p}$ transitively.

By (d), there is an element of $\Delta_{Q}$ which sends $P_{0}=Q \cap A$ to some other subgroup in $\mathcal{S}_{Q}$. We have already seen that the subgroups $P_{1}, \ldots, P_{p}$ all lie in the same $\Delta_{Q}$-orbit, so $P_{0}$ also lies in this orbit, and thus $\Delta_{Q}$ permutes the subgroups in $\mathcal{S}_{Q}$ transitively. In particular, if $P \in \mathcal{S}_{Q}$, and $\varphi \in \Delta_{Q}$ is such that $\varphi\left(P_{0}\right)=P$, then $\varphi c_{g} \varphi^{-1} \in \Delta_{Q}$ is the identity on $P$ and permutes the other subgroups in $\mathcal{S}_{Q}$ transitively.

Proof of (4). Assume $\varphi \in \operatorname{Iso}_{\Delta_{Q}}\left(P, P^{\prime}\right)$ is such that $\varphi(P \cap A)=P^{\prime} \cap A$. We must show that $\varphi \in \operatorname{Iso}_{G}\left(P, P^{\prime}\right)$. Let $\alpha \in \Delta_{Q}$ be such that $\varphi=\left.\alpha\right|_{P}$. If $\alpha(Q \cap A)=Q \cap A$, then the result follows from (c). 
Otherwise, set $Q_{0}=1$ if $Q$ is abelian, and $Q_{0}=Z$ otherwise. Since $\alpha(Q \cap A)$ and $Q \cap A$ are two distinct elements of $\mathcal{S}_{Q}$, and since $\varphi(P \cap A)=P^{\prime} \cap A$,

$$
P^{\prime} \cap A=\alpha(P \cap A) \cap\left(P^{\prime} \cap A\right) \leq \alpha(Q \cap A) \cap(Q \cap A)=Q_{0} .
$$

Since $\left[P^{\prime}: P^{\prime} \cap A\right] \leq p$, we have $\left[P^{\prime} \cdot Q_{0}: Q_{0}\right] \leq p$, and hence either $P^{\prime} \leq Q_{0}$ or $P^{\prime} \cdot Q_{0} \in \mathcal{S}_{Q}$. Also, since $|P \cap A|=\left|P^{\prime} \cap A\right|, P$ and $P^{\prime}$ are either both contained in $A$ or neither is contained in $A$. They cannot both be contained in $A$ if $P^{\prime} \cdot Q_{0} \in \mathcal{S}_{Q}$, since that would imply that $P \cdot Q_{0}=Q \cap A=P^{\prime} \cdot Q_{0}$ and hence that $Q \cap A$ is $\alpha$ invariant. Thus neither $P \cdot Q_{0}$ nor $P^{\prime} \cdot Q_{0}$ can be equal to $Q \cap A$ (either they are both equal to $Q_{0}$ or neither is contained in $A$ ); and since $\alpha\left(Q_{0}\right)=Q_{0}$, this shows that $P^{\prime} \cdot Q_{0}=\alpha(P) \cdot Q_{0}$ is distinct from both $Q \cap A$ and $\alpha(Q \cap A)$. So there is a subgroup $P^{\prime \prime} \in \mathcal{S}_{Q}$, distinct from $Q \cap A$ and $\alpha(Q \cap A)$, and which contains $P^{\prime}$. By (3), there is some $\beta \in \Delta_{Q}$ such that $\left.\beta\right|_{P^{\prime}}=\mathrm{Id}$, and such that $\beta(\alpha(Q \cap A))=Q \cap A$. Thus $\varphi=\left.\beta \alpha\right|_{P}$ and $\beta \alpha(Q \cap A)=Q \cap A$, and $\beta \alpha=c_{x}$ for some $x \in G$ by (c) again.

Proof of (5). Set $r=\operatorname{rk}(Q \cap V) \leq 2$. Thus $Q \cap A=Z V_{r}$ by (2). If $\varphi \in \operatorname{Iso}_{G}\left(P, P^{\prime}\right)$, where $P, P^{\prime} \leq Q$ but are not contained in $A$, then $\varphi=c_{x}$ for some $x \in G$. So $x \in N_{G}(S)$ since $S=P A=P^{\prime} A$ (and $A \triangleleft G$ ), and hence $x$ normalizes $Q \cap A=Z V_{r}$ since $V_{r}$ is the unique subgroup of $V$ of rank $r$ which is normal in $S$. Then $x$ normalizes $Q=P Z V_{r}=P^{\prime} Z V_{r}$, and hence $\varphi$ is the restriction of an element of $\Delta_{Q}$ by $(c)$.

Points (6), (7), and (8). In all of these cases, we are given subgroups $P, P^{\prime} \leq S$ and a morphism $\varphi \in \operatorname{Iso}_{\mathcal{F}}\left(P, P^{\prime}\right)$. Write $\varphi$ as a composite of isomorphisms

$$
P=P_{0} \stackrel{\varphi_{1}}{\cong} P_{1} \underset{\varphi_{2}}{\cong} P_{2} \underset{\cong}{\cong} \underset{\cong}{\cong} P_{k-1} \underset{\varphi_{k}}{\cong} P_{k}=P^{\prime},
$$

where each $\varphi_{i}$ is conjugation by an element of $G$, or is a restriction of an element of $\Delta_{Q}$ for some $Q \in \mathcal{Q}$. We can assume, for each $1 \leq i \leq k-1$, that $P_{i} \leq Q$ for some $Q \in \mathcal{Q}$; otherwise $\varphi_{i}$ and $\varphi_{i+1}$ are both conjugation by elements of $G$ (since they cannot be restrictions of automorphisms in any $\Delta_{Q}$ ), and hence can be replaced by their composite.

In each of the three cases, we will show that we also can assume that

$$
P_{i} \not \leq A \text { for each } i=1, \ldots, k-1 \text {. }
$$

Then for each $2 \leq i \leq k-1, P_{i-1}$ and $P_{i}$ are contained in the same $Q \in \mathcal{Q}$ : by definition if $\varphi_{i} \in \operatorname{Hom}_{\Delta_{Q}}\left(P_{i-1}, P_{i}\right)$, or by point (b) if $\varphi_{i} \in \operatorname{Hom}_{G}\left(P_{i-1}, P_{i}\right)$. Thus $P_{1}, \ldots, P_{k-1}$ are all contained in the same subgroup $Q \in \mathcal{Q}$, and by (5), $\varphi_{i} \in \operatorname{Iso}_{\Delta_{Q}}\left(P_{i-1}, P_{i}\right)$ for each $2 \leq i \leq k-1$.

Proof of (6). We assume here that $P, P^{\prime} \leq Q \in \mathcal{Q}$, and that $P$ and $P^{\prime}$ are not $\mathcal{F}$ conjugate to any subgroup of $A$. Fix $\varphi \in \operatorname{Iso}_{\mathcal{F}}\left(P, P^{\prime}\right)$, and write it as a composite as in $(*)$. Then $(* *)$ holds by assumption, so $P_{i} \leq Q$ for all $i$ by the above remarks, and $\varphi_{i} \in \operatorname{Iso}_{\Delta_{Q}}\left(P_{i-1}, P_{i}\right)$ for all $i$ by $(5)$. Thus $\varphi \in \operatorname{Iso}_{\Delta_{Q}}\left(P, P^{\prime}\right)$.

Proof of (7). Fix $\varphi \in \operatorname{Iso}_{\mathcal{F}}\left(P, P^{\prime}\right)$, decomposed as a composite of the form $(*)$. If $P, P^{\prime} \leq A$, then we can assume that $(* *)$ holds, since otherwise $(*)$ can be split as a composite of chains of this same form. If $k=1$ (i.e., no intermediate groups), then $\varphi=\varphi_{1} \in \operatorname{Iso}_{G}\left(P, P^{\prime}\right)$ by (4). So assume $k>1$, and let $Q \in \mathcal{Q}$ be such that $P_{1}, \ldots, P_{k-1} \leq Q$ and $\varphi_{i} \in \operatorname{Iso}_{\Delta_{Q}}\left(P_{i-1}, P_{i}\right)$ for each $2 \leq i \leq k-1$. Since $P_{1}, P_{k-1} \leq Q, P_{1}, P_{k-1} \not \leq A$, and $P_{0}, P_{k} \leq A$, the morphisms $\varphi_{1}$ and $\varphi_{k}$ cannot be 
conjugation by elements of $G$ and hence are both in $\mathcal{F}_{Q}\left(\Delta_{Q}\right)$. Hence $P, P^{\prime} \leq Q$, $\varphi \in \operatorname{Iso}_{\Delta_{Q}}\left(P, P^{\prime}\right)$, and so $\varphi \in \operatorname{Iso}_{G}\left(P, P^{\prime}\right)$ by $(4)$.

Now assume that $\varphi(P \cap A)=P^{\prime} \cap A$, and that $P, P^{\prime}$ are not $\mathcal{F}$-conjugate to any subgroup of $A$. In particular, $(* *)$ holds, and by the above remarks we can assume that each of the groups $P_{1}, \ldots, P_{k-1}$ is a subgroup of some fixed $Q \in \mathcal{Q}$. If $\varphi_{1}$ is conjugation by an element of $G$, then it sends $P \cap A$ to $P_{1} \cap A$ (since $A \triangleleft G$ ); and since this property is also satisfied by $\varphi$, it is satisfied by the remaining composite $\varphi_{k} \circ \cdots \circ \varphi_{2}$. A similar argument applies to $\varphi_{k}$, and shows that we can assume that neither $\varphi_{1}$ nor $\varphi_{k}$ is conjugation by an element of $G$, since otherwise we could remove them and focus attention on the composite of the other morphisms. Then $\varphi_{1}$ and $\varphi_{k}$ are restrictions of elements of $\Delta_{Q}$ (by (b), the subgroups $P_{1}, P_{k-1}$ cannot be contained in any other element of $\mathcal{Q})$, and hence $P, P^{\prime} \leq Q$. So $\varphi \in \operatorname{Iso}_{\Delta_{Q}}\left(P, P^{\prime}\right)$ by (6), and thus $\varphi \in \operatorname{Iso}_{G}\left(P, P^{\prime}\right)$ by (4).

Proof of (8). We are given $P \leq S$ which is $\mathcal{F}$-conjugate to a subgroup of $A$, and must construct $\psi \in \operatorname{Hom}_{\mathcal{F}}\left(N_{S}(P), S\right)$ such that $\psi(P) \leq A$. This is clear if $P \leq A$ (choose $\psi=\mathrm{Id}$ ). So assume that $P \leq S$ is abelian, not contained in $A$, but $\mathcal{F}$-conjugate to a subgroup $P^{\prime} \leq A$. Choose $\varphi \in \operatorname{Iso}_{\mathcal{F}}\left(P, P^{\prime}\right)$, decomposed as a composite of the form $(*)$. We can assume that $(* *)$ holds, since otherwise we can drop all terms in the chain after the first occurrence of a subgroup $P_{i} \leq A$. As noted above, we can assume that there is some $Q \in \mathcal{Q}$ such that $P_{i} \leq Q$ for all $1 \leq i \leq k-1$ and $\varphi_{i} \in \operatorname{Iso}_{\Delta_{Q}}\left(P_{i-1}, P_{i}\right)$ for all $2 \leq i \leq k-1$. Also, since $P_{k-1} \not \leq A$ and $P_{k} \leq A, \varphi_{k}$ cannot be conjugation by an element of $G$, so $\varphi_{k} \in \operatorname{Iso}_{\Delta_{Q}}\left(P_{k-1}, P_{k}\right)$, and $P_{k} \leq Q$.

Thus, there is a subgroup $P^{\prime \prime} \leq Q\left(P^{\prime \prime}=P_{1}\right.$ or $\left.P_{0}\right)$, together with isomorphisms $\varphi^{\prime \prime} \in \operatorname{Iso}_{G}\left(P, P^{\prime \prime}\right)$ and $\varphi^{\prime} \in \operatorname{Iso}_{\Delta_{Q}}\left(P^{\prime \prime}, P^{\prime}\right)$. Write $\varphi^{\prime \prime}=c_{x}$ for some $x \in G$; then $x$ normalizes $S=P A=P^{\prime \prime} A$ (recall that $A \triangleleft G$ ), so $x\left(N_{S}(P)\right) x^{-1}=N_{S}\left(P^{\prime \prime}\right)$, and $\varphi^{\prime \prime}$ extends to $\psi^{\prime \prime} \in \operatorname{Iso}_{G}\left(N_{S}(P), N_{S}\left(P^{\prime \prime}\right)\right)$. Also, $\varphi^{\prime}$ extends to an automorphism of $Q$, and so we will be done upon showing that $N_{S}\left(P^{\prime \prime}\right) \leq Q$. If $Q$ is elementary abelian of rank 2, then $\left|P^{\prime \prime}\right|=\left|P^{\prime}\right|=p$ (since $P^{\prime \prime} \not \leq A$ and $P^{\prime} \leq A$ are distinct subgroups of $Q$ of the same order), so $P^{\prime \prime} \cap A=1$, and $N_{S}\left(P^{\prime \prime}\right)=P^{\prime \prime} Z=Q$ by (1). Otherwise, $P^{\prime \prime} \cap A \leq Z$ since $P^{\prime \prime}$ is abelian and not contained in $A$, and so $N_{S}\left(P^{\prime \prime}\right) \leq P^{\prime \prime} Z V_{2}=Q$ by (1) again.

Proof of (9). Assume otherwise; i.e., $P \not \leq A$, but $P$ is $\mathcal{F}$-conjugate to some $P^{\prime} \leq A$. Then by (1), and the assumption that $[A: Z] \geq p^{2}$,

$$
\left|C_{S}\left(P^{\prime}\right)\right| \geq|A|>p \cdot|Z| \geq\left|C_{S}(P)\right|,
$$

and so $P$ is not fully centralized in $\mathcal{F}$.

Step 2: We are now ready to prove, using Lemma 1.4, that $\mathcal{F}$ is saturated. We first show that condition $\left(\mathrm{I}^{\prime}\right)$ holds. If $P \leq A$, then by $(7), \operatorname{Aut}_{\mathcal{F}}(P)=\operatorname{Aut}_{G}(P)$, and any other subgroup $P^{\prime} \leq A$ is $\mathcal{F}$-conjugate to $P$ only if it is $G$-conjugate to $P$. Also, by (9), if $P^{\prime} \not \leq A$ is $\mathcal{F}$-conjugate to $P$, then $P^{\prime}$ cannot be fully centralized in $\mathcal{F}$. Thus, by condition (I) applied to the saturated fusion system $\mathcal{F}_{S}(G)$, there is $P^{\prime} \leq A$ which is $G$-conjugate to $P$, fully centralized in $\mathcal{F}_{S}(G)$ (hence in $\mathcal{F}$ ), and such that $\operatorname{Aut}_{S}\left(P^{\prime}\right)$ is a Sylow $p$-subgroup in $\operatorname{Aut}_{\mathcal{F}}\left(P^{\prime}\right)=\operatorname{Aut}_{G}\left(P^{\prime}\right)$. Hence $\left(\mathrm{I}^{\prime}\right)$ holds in this case.

It remains to consider those $P \leq S$ which are not $\mathcal{F}$-conjugate to any subgroup of $A$. Each such subgroup is fully centralized in $\mathcal{F}$, since by $(1),\left|C_{S}\left(P^{\prime}\right)\right|=\left|C_{S}(P)\right|$ 
for all $P^{\prime} \mathcal{F}$-conjugate to $P$. Hence, to prove $\left(\mathrm{I}^{\prime}\right)$, it remains to show that each such subgroup is $\mathcal{F}$-conjugate to some $P^{\prime}$ such that $\operatorname{Aut}_{S}\left(P^{\prime}\right) \in \operatorname{Syl}_{p}\left(\operatorname{Aut}_{\mathcal{F}}\left(P^{\prime}\right)\right)$. If $P$ is not $\mathcal{F}$-conjugate to a subgroup of any $Q \in \mathcal{Q}$, then $\operatorname{Aut}_{\mathcal{F}}(P)=\operatorname{Aut}_{G}(P)$ by definition, and $P$ is $G$-conjugate to a subgroup $P^{\prime}$ such that $\operatorname{Aut}_{S}\left(P^{\prime}\right) \in \operatorname{Syl}_{p}\left(\operatorname{Aut}_{\mathcal{F}}\left(P^{\prime}\right)\right)$ since $\mathcal{F}_{S}(G)$ is saturated. So assume $P \leq Q$ for some $Q \in \mathcal{Q}$. Then by (6), $\operatorname{Aut}_{\mathcal{F}}(P)=\operatorname{Aut}_{\Delta_{Q}}(P)$. Also, by (c), the index of $\operatorname{Aut}_{G}(Q)$ in $\Delta_{Q}=\operatorname{Aut}_{\mathcal{F}}(Q)$ is the order of the $\Delta_{Q}$-orbit of $Q \cap A$, which is equal to $p+1$ by (3). In particular, since $\left|N_{S}\left(Q^{\prime}\right)\right|=\left|N_{S}(Q)\right|$ for all $Q^{\prime} \leq S$ conjugate to $Q, Q$ is fully normalized in $\mathcal{F}_{S}(G)$, and hence

$$
\begin{aligned}
N_{S}(Q) \in \operatorname{Syl}_{p}\left(N_{G}(Q)\right) & \Longrightarrow \operatorname{Aut}_{S}(Q) \in \operatorname{Syl}_{p}\left(\operatorname{Aut}_{G}(Q)\right) \\
& \Longrightarrow \operatorname{Aut}_{S}(Q) \in \operatorname{Syl}_{p}\left(\Delta_{Q}\right) .
\end{aligned}
$$

Thus $\mathcal{F}_{Q \rtimes \operatorname{Aut}_{S}(Q)}\left(Q \rtimes \Delta_{Q}\right)$ is the fusion system of a group. By condition (I) applied to this fusion system, there is $P^{\prime}$ in the $\Delta_{Q}$-orbit of $P$ (hence $\mathcal{F}$-conjugate to $P$ ) such that the group $\left\{\varphi \in \operatorname{Aut}_{S}(Q) \mid \varphi\left(P^{\prime}\right)=P^{\prime}\right\}$ is a Sylow $p$-subgroup of the group $\left\{\varphi \in \Delta_{Q} \mid \varphi\left(P^{\prime}\right)=P^{\prime}\right\}$. Thus $\operatorname{Aut}_{S}\left(P^{\prime}\right) \in \operatorname{Syl}_{p}\left(\operatorname{Aut}_{\Delta_{Q}}\left(P^{\prime}\right)\right)$, and this finishes the proof of $\left(\mathrm{I}^{\prime}\right)$.

Finally, we prove condition (II). Fix $\varphi \in \operatorname{Iso} \mathcal{F}\left(P, P^{\prime}\right)$ such that $P^{\prime}$ is fully centralized in $\mathcal{F}$, and set

$$
N_{\varphi}=\left\{x \in N_{S}(P) \mid \varphi c_{x} \varphi^{-1} \in \operatorname{Aut}_{S}\left(P^{\prime}\right)\right\} .
$$

Set $P_{0}=\varphi^{-1}\left(P^{\prime} \cap A\right)$. By $(8)$, there is $\psi \in \operatorname{Hom}_{\mathcal{F}}\left(N_{S}\left(P_{0}\right), S\right)$ such that $\psi\left(P_{0}\right) \leq A$. Since $P_{0} \triangleleft N_{\varphi}$, we can replace $\varphi$ by $\varphi \circ(\psi \mid P)^{-1}$ and arrange that $\varphi^{-1}\left(P^{\prime} \cap A\right) \leq$ $P \cap A$. This must be an equality, since otherwise $|P \cap A|=|P|=p \cdot\left|P^{\prime} \cap A\right|$, so $P \leq A$ and $P^{\prime} \not A$, and by (9) this contradicts the assumption that $P^{\prime}$ is fully centralized in $\mathcal{F}$. Thus $\varphi(P \cap A)=P^{\prime} \cap A$. Also, by (9) again, either $P, P^{\prime} \leq A$ or they are not $\mathcal{F}$-conjugate to any subgroup of $A$. Hence $\varphi \in \operatorname{Iso}_{G}\left(P, P^{\prime}\right)$ by $(7)$, and this extends to a morphism $\bar{\varphi} \in \operatorname{Hom}_{G}\left(N_{\varphi}, S\right)$ since $\mathcal{F}_{S}(G)$ is saturated.

Proposition 9.1 provides a tool for directly constructing saturated fusion systems and $p$-local finite groups. We will also need to show that the fusion systems we construct (some of them, at least) are not the fusion systems of finite groups. This will be done via reduction to a question about finite simple groups, and then referring to the classification theorem.

If $\mathcal{F}$ is a fusion system over $S$, then a normal subgroup $P \triangleleft S$ will be called strongly closed in $\mathcal{F}$ if no element of $P$ is $\mathcal{F}$-conjugate to any element of $S \backslash P$. A finite group is almost simple if it is an extension of a nonabelian simple group by outer automorphisms. Equivalently, $G$ is almost simple if there is a nonabelian simple subgroup $H \triangleleft G$ such that $C_{G}(H)=1$.

Lemma 9.2. Let $\mathcal{F}$ be a fusion system over a nonabelian p-group $S$ such that $S$ contains no proper strongly closed subgroups. Assume also that $S$ does not factor as a product of two or more subgroups which are permuted transitively by $\operatorname{Aut}_{\mathcal{F}}(S)$. Then if $\mathcal{F}$ is the fusion system of a finite group, it is the fusion system of a finite almost simple group.

Proof. Assume that $\mathcal{F}=\mathcal{F}_{S}(G)$ for some finite group $G$ with $S \in \operatorname{Syl}_{p}(G)$, and that $G$ is a subgroup of minimal order with this property. Let $1 \neq H \triangleleft G$ be a minimal nontrivial normal subgroup. Then $H \cap S$ must be a strongly closed subgroup of $S$, and by assumption either $H \cap S=1$ or $H \cap S=S$. If $H \cap S=1$, then $\mathcal{F}$ is also 
the fusion system of $G / H$, which contradicts the minimality assumption. Hence $S \leq H$. Also, since $H$ is minimal, it is a product of nonabelian simple groups isomorphic to each other [Go, Theorem 2.1.5] which must be permuted transitively by $N_{G}(H)$ (since otherwise $H$ is not minimal). Then $H$ must be simple by the assumption that $S$ does not factor. Thus $C_{G}(H) \cap H=1$, so $C_{G}(H)=1$ by the minimality assumption since $G$ and $G / C_{G}(H)$ have the same fusion system. This shows that $H \triangleleft G \leq \operatorname{Aut}(H)$, and thus that $G$ is almost simple.

We now give some examples of "exotic" $p$-local finite groups which can be constructed using Proposition 9.1. Throughout the rest of the section, we use $v_{p}(n)$ to denote the $p$-adic valuation of an integer $n$. In other words,

$$
v_{p}(n)=a \quad \Longleftrightarrow \quad p^{a} \mid n, p^{a+1} \nmid n .
$$

For each odd prime $p$ and any $\ell \geq 1$, we regard $\left(\mathbb{Z} / p^{\ell}\right)^{p}$ as a $\Sigma_{p}$-representation in the obvious way, and regard $\left(\mathbb{Z} / p^{\ell}\right)^{p-1}$ as the subrepresentation of all $p$-tuples whose sum is zero. When $\ell=1$, the diagonal subspace of $(\mathbb{Z} / p)^{p}$ is contained in $(\mathbb{Z} / p)^{p-1}$ and is fixed by $\Sigma_{p}$. We let $(\mathbb{Z} / p)^{p-2}$ denote the resulting quotient representation. An easy calculation shows that the semidirect product $\left(\mathbb{Z} / p^{\ell}\right)^{p} \rtimes \Sigma_{p}$ contains just one conjugacy class of subgroups of order $p$ not in $\left(\mathbb{Z} / p^{\ell}\right)^{p}$, while for $n=p-1$ or $(n, \ell)=(p-2,1),\left(\mathbb{Z} / p^{\ell}\right)^{n} \rtimes \Sigma_{p}$ contains exactly $p$ conjugacy classes of subgroups of order $p$ not in $\left(\mathbb{Z} / p^{\ell}\right)^{n}$. For example, when $n=p-1$, representatives for these conjugacy classes can be obtained by choosing one such element of order $p$, and conjugating it by a set of coset representatives for $\left(\mathbb{Z} / p^{\ell}\right)^{p-1}$ in $\left(\mathbb{Z} / p^{\ell}\right)^{p}$. More generally, we consider $\left(\mathbb{Z} / p^{\ell}\right)^{p}$ as a representation of the wreath product $C_{p-1}\left\langle\Sigma_{p}\right.$, and $\left(\mathbb{Z} / p^{\ell}\right)^{p-1} \subseteq\left(\mathbb{Z} / p^{\ell}\right)^{p}$ as a representation of $C_{p-1} \times \Sigma_{p}$ (regarded as a subgroup of the wreath product). However, the group $\left(\mathbb{Z} / p^{\ell}\right)^{p-1} \rtimes\left(C_{p-1} \times \Sigma_{p}\right)$ contains only two conjugacy classes of subgroups of order $p$ not in $\left(\mathbb{Z} / p^{\ell}\right)^{p-1}$, since $p-1$ of the conjugacy classes in $\left(\mathbb{Z} / p^{\ell}\right)^{p-1} \rtimes \Sigma_{p}$ fuse in this larger group.

We have chosen this notation since it seems the most natural for describing these groups and representations, although it does lead to mixed additive and multiplicative notation in the groups and fusion systems constructed in the following example.

Example 9.3. Consider the following table:

\begin{tabular}{|c|c|c|c|c|c|}
\hline$A$ & $\Gamma$ & $p$ & $|X|$ & $\Delta_{x}$ & Group \\
\hline$(\mathbb{Z} / p)^{p}$ & $C_{p-1} \prec \Sigma_{p}$ & $p \geq 3$ & 1 & $G L\left(Q_{x}\right)$ & $\Sigma_{p^{2}}$ \\
\hline$\left(\mathbb{Z} / p^{\ell}\right)^{p-1}$ & $C_{p-1} \times \Sigma_{p}$ & $p \geq 5$ & 1,2 & $G L\left(Q_{x}\right)$ & - \\
\hline$(\mathbb{Z} / p)^{p-2}$ & $\Sigma_{p}$ & $p \geq 5$ & $1, \ldots, p$ & $S L\left(Q_{x}\right)$ & $P S L_{p}(q) \quad\left(|X|=p, v_{p}(q-1)=1\right)$ \\
\hline
\end{tabular}

For each pair $(A, \Gamma)$ as given above, where $\Gamma$ is regarded as a group of automorphisms of $A$, set $G=A \rtimes \Gamma$, and fix some $S \in \operatorname{Syl}_{p}(G)$. Let $X$ be a nonempty set of elements of order $p$ in distinct $G$-conjugacy classes in $S \backslash A$, and set $Q_{x}=\langle x, Z(S)\rangle \cong C_{p}^{2}$ for each $x \in X$. Let $\Delta_{x} \leq \operatorname{Aut}\left(Q_{x}\right)$ be as described in the table. Then

$$
\mathcal{F} \stackrel{\text { def }}{=}\left\langle\mathcal{F}_{S}(G) ; \mathcal{F}_{Q_{x}}\left(\Delta_{x}\right) \mid x \in X\right\rangle
$$


is a saturated fusion system over $S$, and has a unique associated centric linking system. Furthermore, $\mathcal{F}$ is not the fusion system of a finite group, except for the cases where otherwise indicated.

Proof. In all cases, the conditions of Proposition 9.1 are satisfied, so $\mathcal{F}$ is a saturated fusion system, and the existence and uniqueness of an associated centric linking system follows from Corollary 3.5 Furthermore, $S$ contains no proper strongly closed subgroups. So by Lemma [9.2, if $\mathcal{F}$ is the fusion system of a finite group, then it must be the fusion system of a finite almost simple group.

Using Proposition 9.5, we see that the only finite almost simple groups which could have such fusion systems are the groups $P S L_{p}(q), P S U_{p}(q)$, and $\Sigma_{p^{2}}$. We leave it as an exercise to show that the first example listed above is the fusion system of $\Sigma_{p^{2}}$, and focus attention on the other cases.

Let $q$ be a prime power such that $p \mid(q-1)$ and $p^{2} \nmid(q-1)$, and regard $G=$ $(\mathbb{Z} / p)^{p-2} \rtimes \Sigma_{p}$ as a subgroup of $P S L_{p}(q)$ in the obvious way. Clearly, $\left[P S L_{p}(q): G\right]$ is prime to $p$, and so any $S \in \operatorname{Syl}_{p}(G)$ is also a Sylow $p$-subgroup of $P S L_{p}(q)$. Fix $z \in \mathbb{F}_{q}^{*}$ of order $p$. Let $Q \leq S$ be the subgroup generated by $\operatorname{diag}\left(1, z, z^{2}, \ldots, z^{p-1}\right)$, together with a permutation matrix in $S$ of order $p$. Let $D_{0}, \ldots, D_{p-1} \in G L_{p}(q)$ be diagonal matrices with $\operatorname{det}\left(D_{i}\right)=z^{i}$, and set $Q_{i}=D_{i} Q D_{i}^{-1}$. Then $Q_{0}, \ldots, Q_{p-1}$ are $G$-conjugacy class representatives for the elementary abelian subgroups of $S$ not contained in $A$ and of rank 2. Set $\Delta_{i}=S L\left(Q_{i}\right)$ : the group of automorphisms of determinant one.

Let $\widetilde{Q}_{i} \leq S L_{p}(q)$ be the inverse image of $Q_{i} \leq P S L_{p}(q)$; these are all extraspecial groups of order $p^{3}$ and exponent $p$. For each $i$,

$$
\operatorname{Aut}_{P S L_{p}(q)}\left(Q_{i}\right) \cong \operatorname{Out}_{S L_{p}(q)}\left(\widetilde{Q}_{i}\right)=\left\{\varphi \in \operatorname{Out}\left(\widetilde{Q}_{i}\right)|\varphi|_{Z\left(\widetilde{Q}_{i}\right)}=\operatorname{Id}\right\} \cong S L_{2}(p),
$$

since for each $\varphi \in \operatorname{Aut}\left(\widetilde{Q}_{i}\right)$ which is the identity on its center, the two irreducible $p$-dimensional $\mathbb{F}_{q}\left[\widetilde{Q}_{i}\right]$-representations defined by the inclusion and by $\varphi$ are isomorphic. Thus $\Delta_{i}=\operatorname{Aut}_{P S L_{p}(q)}\left(Q_{i}\right)$ for each $i$, and hence

$$
\mathcal{F} \stackrel{\text { def }}{=}\left\langle\mathcal{F}_{S}(G) ; \mathcal{F}_{Q_{0}}\left(\Delta_{0}\right), \ldots, \mathcal{F}_{Q_{p-1}}\left(\Delta_{p-1}\right)\right\rangle \subseteq \mathcal{F}_{S}\left(P S L_{p}(q)\right) .
$$

Let $\mathcal{R}$ be the set of subgroups of $S$ which are $p$-centric, $p$-radical, and fully normalized in $\mathcal{F}_{S}\left(P S L_{p}(q)\right)$. By Alperin's fusion theorem (cf. Theorem A.10), all morphisms in $\mathcal{F}_{S}\left(P S L_{p}(q)\right)$ are composites of restrictions of automorphisms of subgroups in $\mathcal{R}$. It is not hard to check that the only elements of $\mathcal{R}$ are $S, A$, and the subgroups conjugate to the $Q_{i}$. Since $N_{P S L_{p}(q)}(S) \leq N_{P S L_{p}(q)}(A)=G$, this shows that $\operatorname{Aut}_{\mathcal{F}}(P)=\operatorname{Aut}_{P S L_{p}(q)}(P)$ for all $P \in \mathcal{R}$. It now follows that $\mathcal{F}=\mathcal{F}_{S}\left(P S L_{p}(q)\right)$.

The following is a slightly more complicated example, also constructed using Proposition 9.1 .

Example 9.4. Fix $p \geq 3$ and $\ell \geq 1$, and set $A=\left(C_{p^{\ell}}\right)^{p}$. Fix $t|s|(p-1)$, and let $\mu_{t} \leq$ $\mu_{s} \leq\left(\mathbb{Z} / p^{\ell}\right)^{*}$ be the cyclic subgroups of order $t$ and $s$, respectively. Let $\Gamma=\Gamma(s, t ; p)$ be the group of permutation matrices in $G L_{p}\left(\mathbb{Z} / p^{\ell}\right) \cong \operatorname{Aut}(A)$ with nonzero entries in $\mu_{s}$ and determinant in $\mu_{t}$. Thus, $\Gamma(s, s ; p) \cong C_{s} \prec \Sigma_{p}$ (the wreath product), and $\Gamma(s, t ; p)$ is a subgroup of index $s / t$ in $\Gamma(s, s ; p)$. Let $\bar{S} \in \operatorname{Syl}_{p}(\Gamma)$ be the subgroup of order $p$ generated by the matrix of the cyclic permutation $(123 \cdots p)$. Set $G=A \rtimes \Gamma$ and $S=A \rtimes \bar{S} \in \operatorname{Syl}_{p}(G)$, and $Z=Z(S)=\left\{(x, x, \ldots, x) \mid x \in C_{p^{\ell}}\right\}$. 
Let $Q \leq S$ be the nonabelian subgroup of order $p^{\ell+2}$ generated by $Z$, together with $\left(1, z, z^{2}, \ldots, z^{p-1}\right)$ for $z \in C_{p^{\ell}}$ of order $p$, and some element in $\bar{S}$ of order $p$. Let $\Delta \cong S L_{2}\left(\mathbb{F}_{p}\right)$ be the group of all automorphisms of $Q$ which are the identity on $Z=Z(Q)$, and set

$$
\mathcal{F}=\mathcal{F}(s, t ; p)=\left\langle\mathcal{F}_{S}(G) ; \mathcal{F}_{Q}(\Delta)\right\rangle .
$$

Then $\mathcal{F}$ is a saturated fusion system over $S$, and has a unique associated centric linking system. If, in addition, $t<\frac{1}{2} s$, then $\mathcal{F}$ is not the fusion system of any finite group.

Proof. The hypotheses of Proposition 9.1 are easily checked, and hence $\mathcal{F}$ is a saturated fusion system. The existence and uniqueness of an associated centric linking system follow from Corollary 3.5. If $\mathcal{F}$ is the fusion system of a finite group $G$, then by Lemma 9.2, $G$ can be chosen to be almost simple. Using Proposition 9.5 below, we now check that if $t<\frac{1}{2} s$, then $\mathcal{F}$ is not the fusion system of any finite group.

If $s=t=1$, and $q$ is a prime power such that $p^{\ell} \mid(q-1)$ but $p^{\ell+1} \nmid(q-1)$, then one can show that $\mathcal{F}(1,1 ; p)$ is isomorphic to the fusion system of $G L_{p}(q)$. The argument is similar to that used in the proof of Example 9.3 to show that a certain fusion system is the fusion system of $P S L_{p}(q)$. Using Table 2 in Proposition 9.5 below, one can find other groups whose fusion systems are isomorphic to $\mathcal{F}(s, s ; p)$ or $\mathcal{F}\left(s, \frac{1}{2} s ; p\right)$ for other values of $s \mid p-1$.

It now remains to list, using the classification theorem, those finite simple groups which have Sylow subgroups of the type encountered above. Recall that a finite abelian $p$-group is homocyclic if it is isomorphic to a product of cyclic groups of the same order.

Proposition 9.5. Fix an odd prime $p$, a finite simple group $G$, and $S \in \operatorname{Syl}_{p}(G)$, such that $S$ is not abelian, but contains an abelian homocyclic subgroup $A$ of rank $r \geq 3$ and index $p$. Then $(G, p, r)$ must be one of the triples listed in Tables 1 and 2. In all cases, $q$ is a prime power prime to $p, \operatorname{ord}_{p}(q)$ is the order of $q$ in the group $\mathbb{F}_{p}^{*}$, and $\ell=v_{p}\left(q^{\operatorname{ord}_{p}(q)}-1\right)$. Table 1 includes all cases except those where $G$ is an alternating group, or a classical group in characteristic $\neq p$.

The remaining cases are covered by Table 2, where we set

$$
k=\operatorname{ord}_{p}(q), \quad k^{\prime}=k /(2, k), \quad \text { and } \quad k^{\prime \prime}=\operatorname{ord}_{p}(-q) .
$$

Also, $\eta U$ means that the group is isomorphic either to $U$ or to an index 2 subgroup of $U$. In all cases in Table $2, A \cong\left(C_{p^{\ell}}\right)^{r}$.

Proof. When $G=A_{n}$, then $\operatorname{rk}_{p}(G)=[n / p]$, and this is an easy exercise. In the other cases, we first look for all simple groups $G$ such that $n \equiv 1(\bmod r)$, where $r=\operatorname{rk}_{p}(G) \geq 3$, and $p^{n}$ is the largest power of $p$ dividing $|G|$.

The $p$-ranks of the other simple groups are given in [GL, 10-1 \& 10-2] for groups of Lie type and characteristic different from $p$, in [GLS, Table 3.3.1] for groups of Lie type and characteristic $p$, and in [GLS, Table 5.6.1] for the sporadic groups. (The $p$-ranks for odd $p$ of the sporadic groups other than $F_{3}$ and $F_{1}$ are also given in [GL p. 123].) Together with the formulas for the orders of the groups (see GLS Table 2.2]), one gets most of the information needed to construct the lists in Tables 1 and 2 . 
TABLE 1.

\begin{tabular}{|c|c|c|c|c|c|}
\hline$G$ & $p$ & $r$ & $A$ & $\operatorname{ord}_{p}(q)$ & $N(A) / C(A)$ \\
\hline$E_{6}(q)$ & 5 & 6 & $\left(C_{p^{\ell}}\right)^{6}$ & 1 & $W\left(E_{6}\right)$ \\
\hline${ }^{2} E_{6}(q)$ & 5 & 6 & $\left(C_{p^{\ell}}\right)^{6}$ & 2 & $W\left(E_{6}\right)$ \\
\hline$E_{7}(q)$ & 5,7 & 7 & $\left(C_{p^{\ell}}\right)^{7}$ & 1,2 & $W\left(E_{7}\right)$ \\
\hline$E_{8}(q)$ & 5 & 4 & $\left(C_{p^{\ell}}\right)^{4}$ & 4 & $\left(C_{4} \times{ }_{C_{2}} D_{8} \times_{C_{2}} D_{8}\right) . S p_{4}(2)$ \\
\hline$E_{8}(q)$ & 7 & 8 & $\left(C_{p^{\ell}}\right)^{8}$ & 1,2 & $W\left(E_{8}\right)$ \\
\hline$P S p_{4}(p)$ & $p$ & 3 & $\left(C_{p}\right)^{3}$ & & $G L_{2}(p) /\{ \pm I\}$ \\
\hline $\mathrm{Co}_{1}$ & 5 & 3 & $\left(C_{5}\right)^{3}$ & & $\left(4 \times A_{5}\right) .2$ \\
\hline
\end{tabular}

TABLE 2 .

\begin{tabular}{|c|c|c|c|}
\hline$G$ & $r$ & Conditions & $N(A) / C(A)$ \\
\hline$A_{n}$ & {$[n / p]$} & $p^{2} \leq n \leq 2 p^{2}-1$ & $\eta C_{p-1}^{r} \rtimes \Sigma_{r}$ \\
\hline$P S L_{n}(q)$ & $\begin{array}{c}p-2 \quad(n=p, \ell=1) \\
n-1 \quad(n>p)\end{array}$ & $k=1, \quad p \leq n \leq 2 p-1$ & $\Sigma_{n}$ \\
\hline$P S L_{n}(q)$ & {$[n / k]$} & $k>1, \quad k p \leq n \leq 2 k p-1$ & $C_{k}^{r} \rtimes \Sigma_{r}$ \\
\hline$P S U_{n}(q)$ & $\begin{array}{c}p-2 \quad(n=p, \ell=1) \\
n-1 \quad(n>p)\end{array}$ & $k=2, \quad p \leq n \leq 2 p-1$ & $\Sigma_{n}$ \\
\hline$P S U_{n}(q)$ & {$\left[n / k^{\prime \prime}\right]$} & $k \neq 2, \quad k^{\prime \prime} p \leq n \leq 2 k^{\prime \prime} p-1$ & $C_{k^{\prime \prime}}^{r} \rtimes \Sigma_{r}$ \\
\hline$P S p_{2 n}(q)$ & {$\left[n / k^{\prime}\right]$} & $k^{\prime} p \leq n \leq 2 k^{\prime} p-1$ & $C_{2 k^{\prime}}^{r} \rtimes \Sigma_{r}$ \\
\hline$P \Omega_{2 n+1}(q)$ & {$\left[n / k^{\prime}\right]$} & $k^{\prime} p \leq n \leq 2 k^{\prime} p-1$ & $C_{2 k^{\prime}}^{r} \rtimes \Sigma_{r}$ \\
\hline$P \Omega_{2 n}^{+}(q)$ & $\begin{array}{c}{\left[n / k^{\prime}\right]-1 \quad k \mid 2 n, k \nmid n} \\
{\left[n / k^{\prime}\right]}\end{array} \quad \begin{array}{c}k^{\prime} p \leq n \leq 2 k^{\prime} p-1 \quad(k \text { odd }) \\
k^{\prime} p+1 \leq n \leq 2 k^{\prime} p-1 \quad(k \text { even })\end{array}$ & $\eta C_{2 k^{\prime}}^{r} \rtimes \Sigma_{r}$ \\
\hline$P \Omega_{2 n}^{-}(q)$ & $\begin{array}{c}{\left[n / k^{\prime}\right]-1 \quad \text { if } k \mid n} \\
{\left[n / k^{\prime}\right]}\end{array}$ & $\begin{array}{c}k^{\prime} p+1 \leq n \leq 2 k^{\prime} p \quad(k \text { odd }) \\
k^{\prime} p \leq n \leq 2 k^{\prime} p\end{array}$ & $\eta C_{2 k^{\prime}}^{r} \rtimes \Sigma_{r}$ even $)$ \\
\hline
\end{tabular}

In particular, among pairs $(G, p)$ such that $G$ is a sporadic simple group or a simple group of Lie type in characteristic $p$, the only cases where $n \equiv 1(\bmod r)$ and $r \geq 3$ (in the above notation) occur for the pairs $\left(\mathrm{Co}_{1}, 5\right),\left(\mathrm{Fi}_{23}, 3\right),\left(F_{2}, 3\right),\left(F_{1}, 5\right)$, 
$\left(P S p_{4}(p), p\right)$, and $\left({ }^{2} A_{6}(p), p\right)$. The groups $\mathrm{Fi}_{23}, F_{2}$, and $F_{1}$ contain extraspecial subgroups of order $3^{9}, 3^{9}$, and $5^{7}$, respectively [GLS, Tables 5.3], and hence their Sylow subgroups do not have abelian normal subgroups of index $p$. One easily checks that a Sylow $p$-subgroup of ${ }^{2} A_{6}(p)=U_{7}(p)$ has no index $p$ abelian subgroup, and so this leaves the two cases listed in the last two rows of Table 1.

When $G=P S p_{4}(p)$, regarded as the (projective) group of isometries of $V=$ $W \oplus W^{*}$, we can take $A$ to be the subgroup of isometries which are the identity on $W$ and on $V / W$, and $N_{G}(A)$ the group of isometries which send $W$ to itself. Thus in $\widetilde{G}=S p_{4}(p)$ we have $N_{\widetilde{G}}(A) / A \cong G L_{2}(p)$, and hence $N_{G}(A) / A \cong G L_{2}(p) /\{ \pm I\}$. When $G=\mathrm{Co}_{1}$, the description of $N_{G}(A) / C_{G}(A)$ is found, for example, in the Atlas Atl. The descriptions of $N(A) / C(A)$ for the groups of exceptional Lie type in Table 1 are given in LSS, Table 5.2].

For the groups of Lie type and characteristic different from $p$, the above argument requires some more explanation. For each Lie "type" $\mathbb{G}$, the order of the universal central extension $\widetilde{\mathbb{G}}(q)$ of $\mathbb{G}(q)$ can be written in the form

$$
|\widetilde{\mathbb{G}}(q)|=q^{N} \cdot \Phi_{1}^{r_{1}}(q) \cdot \Phi_{2}^{r_{2}}(q) \cdots \Phi_{t}^{r_{t}}(q),
$$

where $N$ is the number of positive roots, and $\Phi_{i}$ denotes the $i$-th cyclotomic polynomial. Thus, $\Phi_{i}(X)=\prod(X-\zeta)$, where the product is taken over the primitive $i$-th roots of unity, and

$$
q^{n}-1=\prod_{d \mid n} \Phi_{d}(q) \quad \text { and } \quad q^{n}+1=\prod_{\substack{d \mid 2 n \\ d \nmid n}} \Phi_{d}(q) .
$$

The multiplicities $r_{i}$ are given explicitly in GL, Tables 10-1 \& 10-2], and they also follow easily from any table of the orders of these groups (such as [GLS, Table 2.2]) using the relations in (1). By GL 10-2(2)], for odd $p \nmid q$, the $p$-rank of $\widetilde{\mathbb{G}}(q)$ is equal to $r_{k}$, where $k=\operatorname{ord}_{p}(q)$. More precisely (and with certain listed exceptions of rank 2 ), any Sylow $p$-subgroup of $\widetilde{\mathbb{G}}(q)$ contains a unique maximal abelian subgroup of the form $\left(C_{p^{\ell}}\right)^{r_{k}}$, where $p^{\ell} \mid\left(q^{k}-1\right)$ and $p^{\ell+1} \nmid\left(q^{k}-1\right)$. Thus, $\mathbb{G}(q)$ itself satisfies the hypotheses of the proposition only if the product of the $\Phi_{i}^{r_{i}}(q)$ for $i>k$ is divisible by $p$ but not by $p^{2}$.

Clearly, $p \mid \Phi_{i}(q)$ only if $k \mid i$; and via induction (and relation (1)) one checks that $p \nmid \Phi_{n}(q)$ for $n$ not of the form $k p^{i}$, and that $v_{p}\left(\Phi_{k p^{i}}(q)\right)=1$ for $i \geq 1$. Hence $\mathbb{G}(q)$ satisfies the conditions of the proposition only if

$$
r_{k} \geq 3 \quad \text { and } \quad r_{k p}+r_{k p^{2}}+\cdots=1 .
$$

This condition is easily checked for the nonclassical groups using Table 10-2 in GL, and for the classical groups (with a bit more difficulty) using Table 10-1.

In all cases, $N(A) / C(A)$ is determined by regarding the $A$-representation on the appropriate vector space. For example, all irreducible linear $\mathbb{F}_{q}$-representations of $C_{p}$ are $k$-dimensional, and come from regarding $C_{p}$ as a subgroup of $\mathbb{F}_{q^{k}}$. The centralizer of this subgroup in $G L_{k}(q)$ is $\mathbb{F}_{q^{k}}^{*}$, and the normalizer is $\mathbb{F}_{q^{k}}^{*} \rtimes C_{k}$ (the semidirect product with the group of field automorphisms). Thus, when $A=$ $\left(C_{p}\right)^{r} \leq G=G L_{k r+s}(q)$ and $s<k$, then $N_{G}(A) / C_{G}(A)$ is isomorphic to the wreath product $C_{k}^{r} \rtimes \Sigma_{r}$. Furthermore, elements can be chosen in $C_{G}(A)$ with arbitrary determinant, so $N_{G}(A) / C_{G}(A)$ remains unchanged when we replace $G=G L_{k r+s}(q)$ by $S L_{k r+s}(q)$. 
The arguments for the other classical groups are similar. For example, the minimal $n$ such that $S p_{n}(q)$ or $\Omega_{n}^{ \pm}(q)$ has $p$-torsion is $n=2 k^{\prime}=$ l.c.m. $\{2, k\}$, which is seen by giving $\mathbb{F}_{q^{2 k^{\prime}}}$ an explicit quadratic or symplectic form over $\mathbb{F}_{q^{k^{\prime}}}$. (This can be made into a form over any subfield of $\mathbb{F}_{q^{n}}$ by composing with the trace.) The argument for the unitary groups is similar, where $k^{\prime \prime}=\operatorname{ord}_{p}(-1)$ is the minimal dimension such that $U_{k^{\prime \prime}}(q)$ has $p$-torsion. The determination of $N(A) / C(A)$ for unitary groups is helped by the observation that $S p_{2 n}(q)$ and $O_{n}^{ \pm}(q)$ all occur as subgroups of $U_{n}(q)$.

\section{Appendix A. Properties of SATURATED FUsion systems}

We collect here some results on saturated fusion systems which are needed elsewhere in the paper. All of the results presented here are due to Lluís Puig (see $\mathrm{Pu}$ $\S 1]$ or $\mathrm{Pu} 2, \S \S 2-3])$.

Let $\mathcal{F}$ be a fusion system over a $p$-group $S$. For any subgroup $P \leq S$ and any group of automorphisms $K \leq \operatorname{Aut}(P)$, we set

$$
\operatorname{Aut}_{\mathcal{F}}^{K}(P)=K \cap \operatorname{Aut}_{\mathcal{F}}(P) \quad \text { and } \quad \operatorname{Aut}_{S}^{K}(P)=K \cap \operatorname{Aut}_{S}(P),
$$

and define the $K$-normalizer of $P$ in $S$ to be the subgroup

$$
N_{S}^{K}(P)=\left\{x \in N_{S}(P) \mid c_{x} \in K\right\} .
$$

In particular, $N_{S}^{\operatorname{Aut}(P)}(P)=N_{S}(P)$ is the usual normalizer, and $N_{S}^{\{\operatorname{Id}\}}(P)=C_{S}(P)$ is the centralizer. Also, if $\varphi \in \operatorname{Hom}\left(P, P^{\prime}\right)$ is any monomorphism, we write

$$
\varphi K \varphi^{-1}=\left\{\varphi \chi \varphi^{-1} \mid \chi \in K\right\} \leq \operatorname{Aut}(\varphi(P)) .
$$

Definition A.1. Let $\mathcal{F}$ be any fusion system over $S$. For any $P \leq S$ and any $K \leq$ Aut $(P)$, we say that $P$ is fully $K$-normalized in $\mathcal{F}$ if $\left|N_{S}^{K}(P)\right| \geq\left|N_{S}^{\varphi K \varphi^{-1}}(\varphi(P))\right|$ for all $\varphi \in \operatorname{Hom}_{\mathcal{F}}(P, S)$.

In particular, $P$ is fully centralized in $\mathcal{F}$ if and only if it is fully $\{1\}$-normalized, and is fully normalized in $\mathcal{F}$ if and only if it is fully $\operatorname{Aut}(P)$-normalized. This definition of a fully $K$-normalized subgroup is more restrictive than Puig's definition Pu2, §2.3], but it is equivalent to his definition in the case of saturated fusion systems.

For example, if $\mathcal{F}=\mathcal{F}_{S}(G)$ for some finite group $G$ with Sylow $p$-subgroup $S$, and if $P \leq S$ is a $p$-subgroup of $S$ and $K \leq \operatorname{Aut}(P)$ a subgroup of automorphisms, then $P$ is fully $K$-normalized in $\mathcal{F}_{S}(G)$ if and only if $N_{S}^{K}(P) \in \operatorname{Syl}_{p}\left(N_{G}^{K}(P)\right)$.

Proposition A.2. Let $\mathcal{F}$ be a saturated fusion system over a p-group $S$. Fix subgroups $P \leq S$ and $K \leq \operatorname{Aut}(P)$. Then the following hold:

(a) $P$ is fully $K$-normalized in $\mathcal{F}$ if and only if $P$ is fully centralized in $\mathcal{F}$ and

$$
\operatorname{Aut}_{S}^{K}(P) \in \operatorname{Syl}_{p}\left(\operatorname{Aut}_{\mathcal{F}}^{K}(P)\right)
$$

(b) Fix $\varphi \in \operatorname{Hom}_{\mathcal{F}}(P, S)$, and set $P^{\prime}=\varphi(P)$ and $K^{\prime}=\varphi K \varphi^{-1}$. If $P^{\prime}$ is fully $K^{\prime}$-normalized in $\mathcal{F}$, then there are homomorphisms $\bar{\varphi} \in \operatorname{Hom}_{\mathcal{F}}\left(N_{S}^{K}(P) \cdot P, S\right)$ and $\chi \in K$ such that $\left.\bar{\varphi}\right|_{P}=\varphi \circ \chi$.

Proof. We first prove 
(c) There are a subgroup $P^{\prime} \leq S$ and an isomorphism $\varphi \in \operatorname{Iso}_{\mathcal{F}}\left(P, P^{\prime}\right)$ such that $P^{\prime}$ is fully centralized in $\mathcal{F}$ and

$$
\operatorname{Aut}_{S}^{\varphi K \varphi^{-1}}\left(P^{\prime}\right) \in \operatorname{Syl}_{p}\left(\operatorname{Aut}_{\mathcal{F}}^{\varphi K \varphi^{-1}}\left(P^{\prime}\right)\right) .
$$

To see this, choose $\varphi_{0} \in \operatorname{Hom}_{\mathcal{F}}(P, S)$ such that $P^{\prime} \stackrel{\text { def }}{=} \varphi_{0} P$ is fully normalized. Then, by condition (I) in Definition 1.2 $P^{\prime}$ is fully centralized and $\operatorname{Aut}_{S}\left(P^{\prime}\right) \in$ $\operatorname{Syl}_{p}\left(\operatorname{Aut}_{\mathcal{F}}\left(P^{\prime}\right)\right)$. Hence there is $\alpha \in \operatorname{Aut}_{\mathcal{F}}\left(P^{\prime}\right)$ such that

$$
\begin{aligned}
& \varphi_{0} \operatorname{Aut}_{S}^{K}(P) \varphi_{0}^{-1} \leq\left(\alpha^{-1} \operatorname{Aut}_{S}\left(P^{\prime}\right) \alpha\right) \cap\left(\varphi_{0} \operatorname{Aut}_{\mathcal{F}}^{K}(P) \varphi_{0}^{-1}\right) \\
& \in \operatorname{Syl}_{p}\left(\varphi_{0} \operatorname{Aut}_{\mathcal{F}}^{K}(P) \varphi_{0}^{-1}\right) .
\end{aligned}
$$

So if we set $\varphi=\alpha \circ \varphi_{0}$ and $K^{\prime}=\varphi K \varphi^{-1}$ for short, then

$$
\begin{aligned}
& \operatorname{Aut}_{S}^{K^{\prime}}\left(P^{\prime}\right)=\operatorname{Aut}_{S}\left(P^{\prime}\right) \cap\left(\varphi \operatorname{Aut}_{\mathcal{F}}^{K}(P) \varphi^{-1}\right) \\
& \quad \in \operatorname{Syl}_{p}\left(\varphi \operatorname{Aut}_{\mathcal{F}}^{K}(P) \varphi^{-1}\right)=\operatorname{Syl}_{p}\left(\operatorname{Aut}_{\mathcal{F}}^{K^{\prime}}\left(P^{\prime}\right)\right) .
\end{aligned}
$$

(a) If $P$ is fully centralized in $\mathcal{F}$ and $\operatorname{Aut}_{S}^{K}(P) \in \operatorname{Syl}_{p}\left(\operatorname{Aut}_{\mathcal{F}}^{K}(P)\right)$, then for all $\varphi \in \operatorname{Hom}_{\mathcal{F}}(P, S)$ we have $\left|C_{S}(P)\right| \geq\left|C_{S}(\varphi P)\right|$, and hence

$$
\left|N_{S}^{K}(P)\right|=\left|C_{S}(P)\right| \cdot\left|\operatorname{Aut}_{S}^{K}(P)\right| \geq\left|C_{S}(\varphi P)\right| \cdot\left|\operatorname{Aut}_{S}^{\varphi K \varphi^{-1}}(\varphi P)\right|=\left|N_{S}^{\varphi K \varphi^{-1}}(\varphi P)\right| .
$$

Thus $P$ is fully $K$-normalized in $\mathcal{F}$.

Conversely, assume $P$ is fully $K$-normalized in $\mathcal{F}$. By (c), there is a homomorphism $\varphi \in \operatorname{Hom}_{\mathcal{F}}(P, S)$ such that $\varphi(P)$ is fully centralized in $\mathcal{F}$ and

$$
\operatorname{Aut}_{S}^{\varphi K \varphi^{-1}}(\varphi(P)) \in \operatorname{Syl}_{p}\left(\operatorname{Aut}_{\mathcal{F}}^{\varphi K \varphi^{-1}}(\varphi(P))\right) \text {. }
$$

Thus

$$
\begin{aligned}
\left|C_{S}(P)\right| \cdot\left|\operatorname{Aut}_{S}^{K}(P)\right| & =\left|N_{S}^{K}(P)\right| \geq\left|N_{S}^{\varphi K \varphi^{-1}}(\varphi(P))\right| \\
& =\left|C_{S}(\varphi(P))\right| \cdot\left|\operatorname{Aut}_{S}^{\varphi K \varphi^{-1}}(\varphi(P))\right|,
\end{aligned}
$$

while

$$
\left|C_{S}(P)\right| \leq\left|C_{S}(\varphi(P))\right| \quad \text { and } \quad\left|\operatorname{Aut}_{S}^{K}(P)\right| \leq\left|\operatorname{Aut}_{S}^{\varphi K \varphi^{-1}}(\varphi(P))\right| .
$$

So all of these inequalities are equalities, $P$ is fully centralized, and $\operatorname{Aut}_{S}^{K}(P) \in$ $\operatorname{Syl}_{p}\left(\operatorname{Aut}_{\mathcal{F}}^{K}(P)\right)$.

(b) Now assume that $\varphi \in \operatorname{Hom}_{\mathcal{F}}(P, S)$ is such that $P^{\prime} \stackrel{\text { def }}{=} \varphi(P)$ is fully $K^{\prime} \stackrel{\text { def }}{=}$ $\varphi K \varphi^{-1}$-normalized in $\mathcal{F}$. Clearly $P^{\prime}$ is fully $K^{\prime} \cdot \operatorname{Inn}\left(P^{\prime}\right)$-normalized in $\mathcal{F}$, and so upon replacing $K$ by $K \cdot \operatorname{Inn}(P)$ we can assume that $P \leq N_{S}^{K}(P)$. Since $\operatorname{Aut}_{S}^{K^{\prime}}\left(P^{\prime}\right)$ is a Sylow $p$-subgroup of $\operatorname{Aut}_{\mathcal{F}}^{K^{\prime}}\left(P^{\prime}\right)$, there is some $\chi \in \operatorname{Aut}_{\mathcal{F}}^{K}(P)$ such that

$$
\varphi\left(\chi \operatorname{Aut}_{S}^{K}(P) \chi^{-1}\right) \varphi^{-1} \leq \operatorname{Aut}_{S}^{K^{\prime}}\left(P^{\prime}\right) .
$$

Since $P^{\prime}$ is fully centralized in $\mathcal{F}$, condition (II) now applies to show that $\varphi \circ \chi$ extends to a homomorphism $\bar{\varphi} \in \operatorname{Hom}_{\mathcal{F}}(N, S)$, where

$$
N=N_{\varphi \circ \chi} \stackrel{\text { def }}{=}\left\{g \in N_{S}(P) \mid \varphi \chi c_{g} \chi^{-1} \varphi^{-1} \in \operatorname{Aut}_{S}\left(P^{\prime}\right)\right\} .
$$

Finally, if $g \in N_{S}^{K}(P)$, then $\varphi \chi c_{g} \chi^{-1} \varphi^{-1} \in \operatorname{Aut}_{S}^{K^{\prime}}\left(P^{\prime}\right) \leq \operatorname{Aut}_{S}\left(P^{\prime}\right)$, so $N_{S}^{K}(P) \leq$ $N$, and (b) follows. 
Condition (I) in Definition 1.2 implies that for any saturated fusion system over $S$ we have $\operatorname{Aut}_{S}(S) \in \operatorname{Syl}_{p}\left(\operatorname{Aut}_{\mathcal{F}}(S)\right)$, and hence $\operatorname{Out}_{\mathcal{F}}(S)$ has order prime to $p$. Together with Proposition A.2 this implies that any fusion system which is saturated according to Definition 1.2 is a "full Frobenius system" according to Puig's definition $[\mathrm{Pu}], \mathrm{Pu2}]$. Conversely, if $\mathcal{F}$ is a full Frobenius system over $S$ under Puig's definition, then for each $P \leq S$ and each $K \leq \operatorname{Aut}(P)$, Puig's results together with our Proposition A.2(a) imply that $P$ is fully $K$-normalized in $\mathcal{F}$ if and only if $P$ is fully $K$-normalized in our sense; and using this, one sees that $\mathcal{F}$ is saturated in our sense. So the two definitions are equivalent.

For any $Q \leq S$ and $K \leq \operatorname{Aut}(Q)$, we now consider the $K$-normalizer of $Q$ in $\mathcal{F}$, defined to be a fusion system over the $K$-normalizer $N_{S}^{K}(Q)$ of $Q$ in $S$.

Definition A.3. Let $\mathcal{F}$ be a fusion system over $S$. For each $Q \leq S$ and each $K \leq \operatorname{Aut}(Q)$, let $N_{\mathcal{F}}^{K}(Q)$ (the $K$-normalizer of $Q$ in $\mathcal{F}$ ) be the fusion system over $N_{S}^{K}(Q)$ defined by setting, for all $P, P^{\prime} \leq N_{S}^{K}(Q)$,

$$
\begin{aligned}
& \operatorname{Hom}_{N_{\mathcal{F}}^{K}(Q)}\left(P, P^{\prime}\right) \\
& \quad=\left\{\varphi \in \operatorname{Hom}_{\mathcal{F}}\left(P, P^{\prime}\right)\left|\exists \psi \in \operatorname{Hom}_{\mathcal{F}}\left(P Q, P^{\prime} Q\right), \psi\right|_{P}=\varphi,\left.\psi\right|_{Q} \in K\right\} .
\end{aligned}
$$

In particular, we write $N_{\mathcal{F}}(P)=N_{\mathcal{F}}^{\operatorname{Aut}(P)}(P)$ and $C_{\mathcal{F}}(P)=N_{\mathcal{F}}^{\{\operatorname{Id}\}}(P)$, the normalizer and centralizer of $P$ in $\mathcal{F}$. For example, if $\mathcal{F}=\mathcal{F}_{S}(G)$ for some finite group $G$ and some $S \in \operatorname{Syl}_{p}(G)$, then for any $Q \leq S$,

$$
N_{\mathcal{F}_{S}(G)}(Q)=\mathcal{F}_{N_{S}(Q)}\left(N_{G}(Q)\right) \quad \text { and } \quad C_{\mathcal{F}_{S}(G)}(Q)=\mathcal{F}_{C_{S}(Q)}\left(C_{G}(Q)\right) .
$$

We next show that if $\mathcal{F}$ is a saturated fusion system over $S, Q \leq S$ is fully $K$-normalized in $\mathcal{F}$, and $K \leq \operatorname{Aut}(Q)$, then $N_{\mathcal{F}}^{K}(Q)$ is a saturated fusion system over $N_{S}^{K}(Q)$. Two lemmas will be needed.

Lemma A.4. Let $\mathcal{F}$ be a saturated fusion system over $S$. Fix $P \leq S$ and $K \leq$ $\operatorname{Aut}(P)$ such that $P$ is fully $K$-normalized in $\mathcal{F}$. Then for any

$$
\varphi \in \operatorname{Hom}_{\mathcal{F}}\left(N_{S}^{K}(P) \cdot P, S\right),
$$

$\varphi(P)$ is fully $\varphi K \varphi^{-1}$-normalized in $\mathcal{F}$.

Proof. The homomorphism $\varphi$ sends $N_{S}^{K}(P)$ into $N_{S}^{\varphi K \varphi^{-1}}(\varphi(P))$. In particular,

$$
\left|N_{S}^{\varphi K \varphi^{-1}}(\varphi(P))\right| \geq\left|N_{S}^{K}(P)\right|,
$$

and so $\varphi(P)$ is fully $\varphi K \varphi^{-1}$-normalized in $\mathcal{F}$.

It is not in general true (not even for fusion systems of groups) that if $P \leq S$ is fully $K$-normalized in $\mathcal{F}$, then it is fully $H$-normalized in $\mathcal{F}$ for subgroups $H \leq K$. For example, if we set $G=\Sigma_{4}$, fix any $S \in \operatorname{Syl}_{2}(G)$, let $P \triangleleft G$ be the normal subgroup of order four, and set $K=\operatorname{Aut}_{S^{\prime}}(P)$ for some Sylow 2-subgroup $S^{\prime} \neq S$, then $P$ is fully normalized in $\mathcal{F}_{S}(G)$ but not fully $K$-normalized. However, the next lemma shows that the property of being fully $K$-normalized is inherited by normal subgroups.

Lemma A.5. Let $\mathcal{F}$ be a saturated fusion system over $S$. Let $P \leq S$ and $K \leq$ Aut $(P)$ be such that $P$ is fully $K$-normalized in $\mathcal{F}$. Then for all $H \triangleleft K, P$ is also fully $H$-normalized in $\mathcal{F}$. 
Proof. It is an elementary fact that if $S \in \operatorname{Syl}_{p}(G)$ and $H \triangleleft G$, then $S \cap H \in$ $\operatorname{Syl}_{p}(H)$. By Proposition A.2(a), $P$ is fully centralized and

$$
\operatorname{Aut}_{S}^{K}(P) \in \operatorname{Syl}_{p}\left(\operatorname{Aut}_{\mathcal{F}}^{K}(P)\right) .
$$

Also, $\operatorname{Aut}_{\mathcal{F}}^{H}(P) \triangleleft \operatorname{Aut}_{\mathcal{F}}^{K}(P)$. Hence $\operatorname{Aut}_{S}^{H}(P)=\operatorname{Aut}_{S}^{K}(P) \cap \operatorname{Aut}_{\mathcal{F}}^{H}(P)$ is a Sylow $p$-subgroup of $\operatorname{Aut}_{\mathcal{F}}^{H}(P)$, so $P$ is fully $H$-normalized in $\mathcal{F}$ by Proposition A.2 a) again.

We are now ready to show

Proposition A.6. Let $\mathcal{F}$ be any saturated fusion system over $S$. Fix $Q \leq S$ and $K \leq \operatorname{Aut}(Q)$ such that $Q$ is fully $K$-normalized in $\mathcal{F}$. Then $N_{\mathcal{F}}^{K}(Q)$ is saturated as a fusion system over $N_{S}^{K}(Q)$.

Proof. For each $P \leq N_{S}^{K}(Q)$ and each $I \leq \operatorname{Aut}(P)$, set

$$
I \bullet K=\left\{\alpha \in \operatorname{Aut}(P Q)|\alpha|_{P} \in I,\left.\alpha\right|_{Q} \in K\right\} \leq \operatorname{Aut}(P Q) .
$$

Then

$$
N_{N_{S}^{K}(Q)}^{I}(P)=N_{S}^{I \bullet K}(P Q) \leq N_{S}^{K}(Q),
$$

and the restriction map

$$
\operatorname{Aut}_{\mathcal{F}}^{I \bullet K}(P Q) \stackrel{\text { restr }}{\longrightarrow} \operatorname{Aut}_{N_{\mathcal{F}}^{K}(Q)}^{I}(P)
$$

is surjective.

We will prove that $N_{\mathcal{F}}^{K}(Q)$ is saturated using Lemma 1.4, i.e., by showing that conditions ( $\left.\mathrm{I}^{\prime}\right)$ and (II) hold.

Step 1: We first prove that for each $P \leq N_{S}^{K}(Q)$ and each $I \leq \operatorname{Aut}(P)$, there is some $\varphi \in \operatorname{Hom}_{N_{\mathcal{F}}^{K}(Q)}\left(P Q, N_{S}^{K}(Q)\right)$ such that $\varphi(P Q)$ is fully $\varphi(I \bullet K) \varphi^{-1}$ normalized in $\mathcal{F}$. To see this, choose any $\varphi_{0} \in \operatorname{Hom}_{\mathcal{F}}(P Q, S)$ such that $\varphi_{0}(P Q)$ is fully $\varphi_{0}(I \bullet K) \varphi_{0}^{-1}$-normalized in $\mathcal{F}$, and set $\varphi_{1}=\left.\varphi_{0}\right|_{Q}$ for short. Since $Q$ is fully $K$-normalized in $\mathcal{F}$, there are homomorphisms

$$
\psi \in \operatorname{Hom}_{\mathcal{F}}\left(N_{S}^{\varphi_{1} K \varphi_{1}^{-1}}\left(\varphi_{1}(Q)\right) \cdot \varphi_{1}(Q), S\right) \quad \text { and } \quad \chi \in \varphi_{1} K \varphi_{1}^{-1}
$$

such that $\left.\psi\right|_{\varphi_{1}(Q)}=\varphi_{1}^{-1} \circ \chi($ Proposition $\mathrm{A.2}(\mathrm{b}))$. Then

$$
\left.\psi\right|_{\varphi_{1}(Q)}=\chi^{\prime} \circ \varphi_{1}^{-1}, \quad \text { where } \quad \chi^{\prime}=\varphi_{1}^{-1} \circ \chi \circ \varphi_{1} \in K .
$$

Set $\varphi=\psi \circ \varphi_{0}$. Then $\varphi \in \operatorname{Hom}_{N_{\mathcal{F}}^{K}(Q)}\left(P Q, N_{S}^{K}(Q)\right)$, since $\varphi(Q)=Q$ and $\left.\varphi\right|_{Q}=$ $\chi^{\prime} \in K$. Finally, by Lemma A.4 $\varphi(P Q)$ is fully $\varphi(I \bullet K) \varphi^{-1}$-normalized in $\mathcal{F}$.

Step 2: We now prove condition $\left(\mathrm{I}^{\prime}\right)$ in Lemma 1.4. We need to show that for each $P \leq N_{S}^{K}(Q)$, there is some morphism $\varphi \in \operatorname{Hom}_{N_{\mathcal{F}}^{K}(Q)}\left(P, N_{S}^{K}(Q)\right)$ such that $\varphi(P)$ is fully centralized in $N_{\mathcal{F}}^{K}(Q)$ and $\operatorname{Aut}_{N_{S}^{K}(Q)}(\varphi(P)) \in \operatorname{Syl}_{p}\left(\operatorname{Aut}_{N_{\mathcal{F}}^{K}(Q)}(\varphi(P))\right)$.

Write $K_{P}=\operatorname{Aut}(P) \bullet K$ for short. By Step 1, there is

$$
\varphi \in \operatorname{Hom}_{N_{\mathcal{F}}^{K}(Q)}\left(P Q, N_{S}^{K}(Q)\right)
$$

such that $\varphi(P Q)$ is fully $\varphi K_{P} \varphi^{-1}$-normalized in $\mathcal{F}$. In particular, since $(1 \bullet K) \triangleleft$ $K_{P}$, Lemma A.5 implies that $\varphi(P Q)$ is fully $\varphi(1 \bullet K) \varphi^{-1}$-normalized in $\mathcal{F}$. Furthermore, $\varphi(1 \bullet K) \varphi^{-1}=1 \bullet K$ as subgroups of $\operatorname{Aut}(\varphi(P Q))$, where "1" denotes the 
trivial subgroup first of $\operatorname{Aut}(P)$ and then of $\operatorname{Aut}(\varphi(P))$. Equation (1), applied to $\varphi(P)$ and with $I=1$, thus implies that

$$
C_{N_{S}^{K}(Q)}(\varphi(P))=N_{S}^{1 \bullet K}(\varphi(P Q))=N_{S}^{\varphi(1 \bullet K) \varphi^{-1}}(\varphi(P Q)) .
$$

For any other $\psi \in \operatorname{Hom}_{N_{\mathcal{F}}^{K}(Q)}\left(P Q, N_{S}^{K}(Q)\right)$ we have

$$
C_{N_{S}^{K}(Q)}(\psi(P))=N_{S}^{\psi(1 \bullet K) \psi^{-1}}(\psi(P Q))
$$

by the same argument, and hence

$$
\begin{aligned}
\left|C_{N_{S}^{K}(Q)}(\psi(P))\right| & =\left|N_{S}^{\psi(1 \bullet K) \psi^{-1}}(\psi(P Q))\right| \\
& \leq\left|N_{S}^{\varphi(1 \bullet K) \varphi^{-1}}(\varphi(P Q))\right| \\
& =\left|C_{N_{S}^{K}(Q)}(\varphi(P))\right|
\end{aligned}
$$

since $\varphi(P Q)$ is fully $\varphi(1 \bullet K) \varphi^{-1}$-normalized in $\mathcal{F}$; and thus $\varphi(P)$ is fully centralized in $N_{\mathcal{F}}^{K}(Q)$.

Since $\varphi(P Q)$ is fully $\varphi K_{P} \varphi^{-1}$-normalized in $\mathcal{F}$,

$$
\operatorname{Aut}_{S}^{\varphi K_{P} \varphi^{-1}}(\varphi(P Q)) \in \operatorname{Syl}_{p}\left(\operatorname{Aut}_{\mathcal{F}}^{\varphi K_{P} \varphi^{-1}}(\varphi(P Q))\right)
$$

by Proposition A.2(b). Also, $N_{N_{S}^{K}(Q)}(\varphi(P))=N_{S}^{\varphi K_{P} \varphi^{-1}}(\varphi(P Q))$ by (1) (and since $\left.\varphi K_{P} \varphi^{-1}=\operatorname{Aut}(\varphi(P)) \bullet K\right)$; and hence

$$
\operatorname{Aut}_{N_{S}^{K}(Q)}(\varphi(P)) \in \operatorname{Syl}_{p}\left(\operatorname{Aut}_{N_{\mathcal{F}}^{K}(Q)}(\varphi(P))\right)
$$

by (2). We have already shown that $\varphi(P)$ is fully centralized in $N_{\mathcal{F}}^{K}(Q)$, and so this finishes the proof of condition $\left(\mathrm{I}^{\prime}\right)$.

Step 3: It remains to prove condition (II). We first claim that

(3) $P$ is fully $I$-normalized in $N_{\mathcal{F}}^{K}(Q) \Longrightarrow P Q$ is fully $(I \bullet K)$-normalized in $\mathcal{F}$.

To see this, assume $P$ is fully $I$-normalized in $N_{\mathcal{F}}^{K}(Q)$, and use Step 1 to choose $\varphi \in \operatorname{Hom}_{N_{\mathcal{F}}^{K}(Q)}\left(P Q, N_{S}^{K}(Q)\right)$ such that $\varphi(P Q)$ is fully $\varphi(I \bullet K) \varphi^{-1}$-normalized in $\mathcal{F}$. Then

$$
\begin{aligned}
\left|N_{S}^{I \bullet K}(P Q)\right|=\left|N_{N_{S}^{K}(Q)}^{I}(P)\right| & \geq\left|N_{N_{S}^{K}(Q)}^{\varphi I \varphi^{-1}}(\varphi(P))\right| \\
& =\left|N_{S}^{\left(\varphi I \varphi^{-1}\right) \bullet K}(\varphi(P) \cdot Q)\right|=\left|N_{S}^{\varphi(I \bullet K) \varphi^{-1}}(\varphi(P Q))\right|,
\end{aligned}
$$

where the three equalities hold by (1) and since $\left.\varphi\right|_{Q} \in K$, and the inequality holds since $P$ is fully $I$-normalized. So $P Q$ is fully $(I \bullet K)$-normalized in $\mathcal{F}$ since $\varphi(P Q)$ is fully $\varphi(I \bullet K) \varphi^{-1}$-normalized in $\mathcal{F}$.

Now fix $\varphi \in \operatorname{Hom}_{N_{\mathcal{F}}^{K}(Q)}\left(P, N_{S}^{K}(Q)\right)$, and assume that $\varphi(P)$ is fully centralized in $N_{\mathcal{F}}^{K}(Q)$. Set

$$
N_{\varphi}=\left\{g \in N_{N_{S}^{K}(Q)}(P) \mid \varphi c_{g} \varphi^{-1} \in \operatorname{Aut}_{N_{S}^{K}(Q)}(\varphi(P))\right\} \quad \text { and } \quad I=\operatorname{Aut}_{N_{\varphi}}(P) .
$$

Then

and

$$
I=\left\{\alpha \in \operatorname{Aut}_{N_{S}^{K}(Q)}(P) \mid \varphi \alpha \varphi^{-1} \in \operatorname{Aut}_{N_{S}^{K}(Q)}(\varphi(P))\right\}
$$

$$
N_{\varphi}=N_{N_{S}^{K}(Q)}^{I}(P)=N_{S}^{I \bullet K}(P Q) .
$$

Set $I^{\prime}=\varphi I \varphi^{-1} \leq \operatorname{Aut}_{N_{S}^{K}(Q)}(\varphi(P))$. Then

$$
I^{\prime} \leq \operatorname{Aut}_{N_{S}^{K}(Q)}(\varphi(P)) \leq \operatorname{Aut}_{N_{\mathcal{F}}^{K}(Q)}(\varphi(P))
$$


and thus

$$
\operatorname{Aut}_{N_{S}^{K}(Q)}^{I^{\prime}}(\varphi(P))=I^{\prime}=\operatorname{Aut}_{N_{\mathcal{F}}^{K}(Q)}^{I^{\prime}}(\varphi(P)) .
$$

Since $\varphi(P)$ is fully centralized in $N_{\mathcal{F}}^{K}(Q)$, this (together with Proposition A.2 (a)) shows that it is fully $I^{\prime}$-normalized in $N_{\mathcal{F}}^{K}(Q)$. Hence $\varphi(P) \cdot Q$ is fully $I^{\prime} \bullet K$ normalized in $\mathcal{F}$ by $(3)$. By definition of $N_{\mathcal{F}}^{K}(Q)$, there exists $\widehat{\varphi} \in \operatorname{Hom}_{\mathcal{F}}(P Q, S)$ such that $\left.\widehat{\varphi}\right|_{P}=\varphi$ and $\left.\widehat{\varphi}\right|_{Q} \in K$. Also, $I^{\prime} \bullet K=\widehat{\varphi}(I \bullet K) \widehat{\varphi}^{-1}$, and hence by Proposition A.2(b) there are homomorphisms

$$
\bar{\varphi} \in \operatorname{Hom}_{\mathcal{F}}\left(N_{S}^{I \bullet K}(P Q) \cdot P Q, S\right) \quad \text { and } \quad \chi \in I \bullet K
$$

such that $\left.\bar{\varphi}\right|_{P Q}=\widehat{\varphi} \circ \chi$. Set $\varphi_{0}=\left.\bar{\varphi}\right|_{N_{\varphi}}$. Then $\left.\varphi_{0}\right|_{Q}=\left.\widehat{\varphi} \circ \chi\right|_{Q} \in K$ (since $\left.\left.\hat{\varphi}\right|_{Q},\left.\chi\right|_{Q} \in K\right)$, and hence $\operatorname{Im}\left(\varphi_{0}\right) \leq N_{S}^{K}(Q)$. Since $\left.\chi\right|_{P} \in I=\operatorname{Aut}_{N_{\varphi}}(P)$, there is some $g \in N_{\varphi}$ such that $\left.\chi\right|_{P}=c_{g}$; and $\varphi=\left.\varphi_{0} \circ \chi^{-1}\right|_{P}$ thus extends to

$$
\varphi_{0} \circ c_{g}^{-1} \in \operatorname{Hom}_{N_{\mathcal{F}}^{K}(Q)}\left(N_{\varphi}, N_{S}^{K}(Q)\right) .
$$

This finishes the proof of condition (II).

Recall, for any fusion system $\mathcal{F}$ over a $p$-group $S$, that a subgroup $P \leq S$ is called $\mathcal{F}$-centric if $C_{S}(\varphi(P))=Z(\varphi(P))$ for each $\varphi \in \operatorname{Hom}_{\mathcal{F}}(P, S)$. In other words, $P \leq S$ is $\mathcal{F}$-centric if each subgroup in the $\mathcal{F}$-conjugacy class of $P$ contains its $S$-centralizer. In particular, if $P$ is $\mathcal{F}$-centric, then every $Q \leq S$ containing $P$ is $\mathcal{F}$-centric; and hence every $Q \leq S$ such that $\operatorname{Hom}_{\mathcal{F}}(P, Q) \neq \emptyset$ is $\mathcal{F}$-centric.

Lemma A.7. Let $\mathcal{F}$ be a fusion system over a p-group $S$. Then every $\mathcal{F}$-centric subgroup $P \leq S$ is fully centralized in $\mathcal{F}$. Conversely, if $P \leq S$ is fully centralized in $\mathcal{F}$, then $C_{S}(P) \cdot P$ is $\mathcal{F}$-centric.

Proof. The first claim is immediate, since if $P$ is $\mathcal{F}$-centric, then the centralizers in $S$ of $\mathcal{F}$-conjugates of $P$ are all isomorphic.

To prove the converse, set $Q=C_{S}(P) \cdot P$ for short. We must show that $C_{S}(\varphi(Q))$ $=Z(\varphi(Q))$ for all $\varphi \in \operatorname{Hom}_{\mathcal{F}}(Q, S)$. Fix such a $\varphi$, and set $Q^{\prime}=\varphi(Q)$ and $P^{\prime}=$ $\varphi(P) \leq Q^{\prime}$. Then $\varphi\left(C_{S}(P)\right) \leq C_{S}\left(P^{\prime}\right)$, and since $P$ is fully centralized in $\mathcal{F}$ this must be an equality. Hence $Q^{\prime}=C_{S}\left(P^{\prime}\right) \cdot P^{\prime}$, so $C_{S}\left(Q^{\prime}\right) \leq C_{S}\left(P^{\prime}\right) \leq Q^{\prime}$, and $C_{S}\left(Q^{\prime}\right)=Z\left(Q^{\prime}\right)$.

If $\mathcal{F}=\mathcal{F}_{S}(G)$ is the fusion system of a finite group $G$ over a Sylow $p$-subgroup $S \leq G$, then a subgroup $P \leq S$ is $\mathcal{F}$-centric if and only if $P$ is $p$-centric in $G$, i.e., if and only if $Z(P) \in \operatorname{Syl}_{p}\left(C_{G}(P)\right)$. This follows immediately from the observation that for any $P \leq S$, one has $C_{S}\left(P^{\prime}\right) \in \operatorname{Syl}_{p}\left(C_{G}\left(P^{\prime}\right)\right)$ for some $P^{\prime}$ conjugate to $P$ in $G$ (see [BLO, Lemma A.5]).

The following proposition gives one important property of $\mathcal{F}$-centric subgroups.

Proposition A.8. Let $\mathcal{F}$ be a saturated fusion system over the p-group $S$. Then for each $\mathcal{F}$-centric subgroup $P \leq S$, each $P \leq Q \leq S$, and each $\varphi, \varphi^{\prime} \in \operatorname{Hom}_{\mathcal{F}}(Q, S)$ such that $\left.\varphi\right|_{P}=\left.\varphi^{\prime}\right|_{P}$, there is some $g \in Z(P)$ such that $\varphi^{\prime}=\varphi \circ c_{g}$.

Proof. Assume first that $P \triangleleft Q$. Then for each $x \in Q, c_{\varphi(x)}$ and $c_{\varphi^{\prime}(x)}$ are equal on $\varphi(P)=\varphi^{\prime}(P)$, and thus $\varphi(x) \equiv \varphi^{\prime}(x)$ modulo $C_{S}(\varphi(P))$, and $C_{S}(\varphi(P)) \leq \varphi(P)$ (since $P$ is $\mathcal{F}$-centric). In particular, this shows that $\varphi(Q)=\varphi^{\prime}(Q)$. So upon replacing $\varphi$ by $\left(\varphi^{\prime}\right)^{-1} \circ \varphi$, we can assume that $\varphi \in \operatorname{Aut}_{\mathcal{F}}(Q)$ and $\left.\varphi\right|_{P}=\operatorname{Id}_{P}$.

Set $K=\left\{\chi \in \operatorname{Aut}(Q)|\chi|_{P}=\operatorname{Id}_{P}\right\}$. Since $C_{Q}(P) \leq P$, each $\chi \in K$ must induce the identity on $Q / P$, and hence $K$ is a $p$-group (cf. Go, Corollary 5.3.3]). We 
can assume that $Q$ is fully $K$-normalized in $\mathcal{F}$ : otherwise replace it by some other subgroup in the same $\mathcal{F}$-conjugacy class. Then $\operatorname{Aut}_{S}^{K}(Q)=\operatorname{Aut}_{\mathcal{F}}^{K}(Q)$ since $K$ is a p-group. So $\varphi=c_{g}$ for some $g \in N_{S}(Q)$, and $g \in C_{S}(P)=Z(P)$ since $\left.\varphi\right|_{P}=\mathrm{Id}$.

If $P$ is not normal in $Q$, then there is a subnormal sequence $P=P_{0} \triangleleft P_{1} \triangleleft$ $\cdots \triangleleft P_{k}=Q$, and hence elements $g_{i} \in Z\left(P_{i-1}\right) \leq Z(P)$ such that $\left.\varphi^{\prime}\right|_{P_{i}}=$ $\left.\left(\varphi \circ c_{g_{1}} \circ \cdots \circ c_{g_{i}}\right)\right|_{P_{i}}$ for each $1 \leq i \leq k$.

If $G$ is a finite group then a $p$-subgroup $P \leq G$ is called $p$-radical if $N_{G}(P) / P$ is $p$-reduced, namely, if $O_{p}\left(N_{G}(P) / P\right)=1$. Here, as usual, $O_{p}(-)$ denotes the maximal normal $p$-subgroup. Radical subgroups can also be defined in the context of fusion systems.

Definition A.9. For any fusion system $\mathcal{F}$ over a $p$-group $S$, a $\operatorname{subgroup} P \leq S$ is called $\mathcal{F}$-radical if $\operatorname{Out}_{\mathcal{F}}(P)$ is $p$-reduced, i.e., if $O_{p}\left(\operatorname{Out}_{\mathcal{F}}(P)\right)=1$.

Note that when $G$ is a finite group, $S \in \operatorname{Syl}_{p}(G)$, and $\mathcal{F}=\mathcal{F}_{S}(G)$, then a subgroup $P \leq S$ is $\mathcal{F}$-radical when $O_{p}\left(N_{G}(P) / P \cdot C_{G}(P)\right)=1$, while $P$ is $p$-radical when $O_{p}\left(N_{G}(P) / P\right)=1$. In general, these two conditions are independant. If, however, $P$ is $p$-centric (equivalently $\mathcal{F}$-centric) and $\mathcal{F}$-radical, then it is also $p$ radical.

The following is one version of Alperin's fusion theorem for saturated fusion systems, one which suffices for our purposes here. A stronger version has been shown by Puig [Pu2, Corollary 3.9].

Theorem A.10 (Alperin's fusion theorem for saturated fusion systems). Let $\mathcal{F}$ be a saturated fusion system over $S$. Then for each morphism $\varphi \in \operatorname{Iso}_{\mathcal{F}}\left(P, P^{\prime}\right)$ in $\mathcal{F}$, there exist sequences of subgroups of $S$

$$
P=P_{0}, P_{1}, \ldots, P_{k}=P^{\prime} \quad \text { and } \quad Q_{1}, Q_{2}, \ldots, Q_{k},
$$

and elements $\varphi_{i} \in \operatorname{Aut}_{\mathcal{F}}\left(Q_{i}\right)$, such that

(a) $Q_{i}$ is fully normalized in $\mathcal{F}, \mathcal{F}$-radical, and $\mathcal{F}$-centric for each $i$;

(b) $P_{i-1}, P_{i} \leq Q_{i}$ and $\varphi_{i}\left(P_{i-1}\right)=P_{i}$ for each $i$; and

(c) $\varphi=\varphi_{k} \circ \varphi_{k-1} \circ \cdots \circ \varphi_{1}$.

Proof. By downward induction on the order of $P$. The claim is clear for $P=S$.

Assume $P \supsetneqq S$. Let $P^{*} \leq S$ be any subgroup which is $\mathcal{F}$-conjugate to $P$ and fully normalized in $\mathcal{F}$, and fix $\psi \in \operatorname{Iso}_{\mathcal{F}}\left(P, P^{*}\right)$. The theorem holds for $\varphi \in \operatorname{Iso}_{\mathcal{F}}\left(P, P^{\prime}\right)$ if it holds for $\psi$ and for $\psi \circ \varphi^{-1} \in \operatorname{Iso}_{\mathcal{F}}\left(P^{\prime}, P^{*}\right)$. So we are thus reduced to proving the theorem when the target group $P^{\prime}$ is fully normalized in $\mathcal{F}$.

Since $P^{\prime}$ is fully normalized, there are homomorphisms $\bar{\varphi} \in \operatorname{Hom}_{\mathcal{F}}\left(N_{S}(P), S\right)$ and $\chi \in \operatorname{Aut}_{\mathcal{F}}(P)$ such that $\bar{\varphi}(P)=P^{\prime}$ and $\varphi=\left.\bar{\varphi}\right|_{P} \circ \chi^{-1}$ (Proposition A.2(b)). Since $N_{S}(P) \supsetneqq P$ (since $P \supsetneqq S$ ), the theorem holds for $\bar{\varphi}$ (as an isomorphism to its image) by the induction hypothesis. So it holds for $\varphi$ if and only if it holds for $\chi$. Hence it now remains only to prove it when $P=P^{\prime}$ is fully normalized in $\mathcal{F}$ and $\varphi \in \operatorname{Aut}_{\mathcal{F}}(P)$.

In particular, $P$ is fully centralized in $\mathcal{F}$ by condition $(\mathrm{I})$ in Definition 1.2 . So if $P$ is not $\mathcal{F}$-centric, then by condition (II) in Definition $1.2 \varphi$ extends to a morphism $\bar{\varphi} \in \operatorname{Hom}_{\mathcal{F}}\left(C_{S}(P) \cdot P, S\right)$. Clearly, $\operatorname{Im}(\bar{\varphi})=C_{S}(P) \cdot P$, so we can regard this as an automorphism $\bar{\varphi} \in \operatorname{Aut}_{\mathcal{F}}\left(C_{S}(P) \cdot P\right)$. Since $C_{S}(P) \cdot P \supsetneqq P$, the theorem holds for $\varphi$ by the induction hypothesis. 
Now assume that $P$ is not $\mathcal{F}$-radical. Set $K=O_{p}\left(\operatorname{Aut}_{\mathcal{F}}(P)\right) \supsetneqq \operatorname{Inn}(P)$. Since $P$ is fully normalized in $\mathcal{F}, \operatorname{Aut}_{S}(P) \in \operatorname{Syl}_{p}\left(\operatorname{Aut}_{\mathcal{F}}(P)\right)$, and so $K \leq \operatorname{Aut}_{S}(P)$. In particular, $N_{S}^{K}(P) \supsetneqq P$ since $K \supsetneqq \operatorname{Inn}(P)$. Also, for each $g \in N_{S}^{K}(P)$ we have $\varphi c_{g} \varphi^{-1} \in K \leq \operatorname{Aut}_{S}(P)$ since $K \triangleleft \operatorname{Aut}_{\mathcal{F}}(P)$. So by condition (II) again, $\varphi$ extends to an automorphism of $N_{S}^{K}(P)$, and the theorem again holds for $\varphi$ by the induction hypothesis.

Finally, if $\varphi \in \operatorname{Aut}_{\mathcal{F}}(P)$ and $P$ is a fully normalized $\mathcal{F}$-centric $\mathcal{F}$-radical subgroup of $S$, then the theorem holds for trivial reasons.

\section{REFERENCES}

[Alp] J. Alperin, Local representation theory, Cambridge Univ. Press (1986). MR 87i:20002

[AB] J. Alperin \& M. Broué, Local methods in block theory, Annals of Math. 110 (1979), 143-157. MR 80f:20010

[AT] E. Artin \& J. Tate, A note on finite ring extensions, J. Math. Soc. Japan 3 (1951), 74-77. MR 13:427c

[AM] M. Atiyah \& I. Macdonald, Introduction to commutative algebra, Adison-Wesley (1969). MR 39:4129

[Be] D. Benson, Cohomology of sporadic groups, finite loop spaces, and the Dickson invariants, Geometry and cohomology in group theory, London Math. Soc. Lecture Notes Ser. 252, Cambridge Univ. Press (1998), 10-23. MR 2001i:55017

[BK] P. Bousfield \& D. Kan, Homotopy limits, completions, and localizations, Lecture notes in math. 304, Springer-Verlag (1972). MR 51:1825

[Bd] G. Bredon, Sheaf theory, McGraw Hill (1967). MR 36:4552

[BrK] C. Broto \& N. Kitchloo, Classifying spaces of Kac-Moody groups, Math. Z. 240 (2002), 621-649.

[BL] C. Broto \& R. Levi, On spaces of self homotopy equivalences of $p$-completed classifying spaces of finite groups and homotopy group extensions, Topology 41 (2002), 229-255. MR 2002j:55013

[BLO] C. Broto, R. Levi, \& B. Oliver, Homotopy equivalences of $p$-completed classifying spaces of finite groups, Invent. Math. 151 (2003), 611-664.

[BrM] C. Broto \& J. Møller, Homotopy finite Chevalley versions of $p$-compact groups, preprint, 2003.

[Bw] K. Brown, Cohomology of groups, Springer-Verlag (1982). MR 83k:20002

[Atl] J. Conway, R. Curtis, S. Norton, R. Parker, \& R. Wilson, Atlas of finite groups, Oxford Univ. Press (1985). MR 88g:20025

[Dw] W. Dwyer, The centralizer decomposition of $B G$, in: Algebraic topology: new trends in localization and periodicity, Progr. Math. 136, Birkhäuser (1996), 167-184. MR 97i:55028

[DK] W. Dwyer \& D. Kan, Realizing diagrams in the homotopy category by means of diagrams of simplicial sets, Proc. Amer. Math. Soc. 91 (1984), 456-460. MR 86c:55010b

[DK2] W. Dwyer \& D. Kan, Centric maps and realizations of diagrams in the homotopy category, Proc. Amer. Math. Soc. 114 (1992), 575-584. MR 92e:55011

[DW1] W. Dwyer \& C. Wilkerson, A cohomology decomposition theorem, Topology 31 (1992), 433-443. MR 93h:55008

[EP] L. Evens \& S. Priddy, The ring of universally stable elements, Quart. J. Math. Oxford 40 (1989), 399-407. MR 90k:20089

[GZ] P. Gabriel \& M. Zisman, Calculus of fractions and homotopy theory, Springer-Verlag (1967). MR 35:1019]

[GJ] P. Goerss \& J. Jardine, Simplicial homotopy theory, Birkhäuser (1999). MR 2001d:55012

[Go] D. Gorenstein, Finite groups, Harper \& Row (1968). MR 38:229

[GL] D. Gorenstein \& R. Lyons, The local structure of finite groups of characteristic 2 type, Memoirs Amer. Math. Soc. 276 (1983). MR 84g:20025

[GLS] D. Gorenstein, R. Lyons, \& R. Solomon, The classification of the finite simple groups, Number 3, Math. Surveys and Monographs, vol. 40.3, Amer. Math. Soc. (1998). MR 98j:20011

[Gr] J. Grodal, Higher limits via subgroup complexes, Annals of Math. 155 (2002), 405-457. MR 2003g:55025 
[Hf] G. Hoff, Cohomologies et extensions de categories, Math. Scand. 74 (1994), 191-207. MR 95k:18012

[HV] J. Hollender \& R. Vogt, Modules of topological spaces, applications to homotopy limits and $E_{\infty}$ structures, Arch. Math. 59 (1992), 115-129. MR 93e:55015

[JM] S. Jackowski \& J. McClure, Homotopy decomposition of classifying spaces via elementary abelian subgroups, Topology 31 (1992), 113-132. MR 92k:55026

[JMO] S. Jackowski, J. McClure, \& B. Oliver, Homotopy classification of self-maps of $B G$ via $G$-actions, Annals of Math. 135 (1992), 183-270. MR 93e:55019a

[JMO2] S. Jackowski, J. McClure, \& B. Oliver, Homotopy theory of classifying spaces of compact Lie groups, Algebraic topology and its applications, M.S.R.I. Publ. 27, Springer-Verlag (1994), 81-123. MR 95b:55001

[La] J. Lannes, Sur les espaces fonctionnels dont la source est le classifiant d'un $p$-groupe abélien élémentaire, Publ. Math. I.H.E.S. 75 (1992), 135-244. MR 93j:55019

[LS] J. Lannes \& L. Schwartz, Sur la structure des $A$-modules instables injectifs, Topology 28 (1989), 153-169. MR 90h:55027

[LSS] M. Liebeck, J. Saxl, \& G. Seitz, Subgroups of maximal rank in finite exceptional groups of Lie type, Proc. London Math. Soc. 65 (1992), 297-325. MR 93e:20026

[LW] M. Linckelmann \& P. Webb, unpublished notes.

[Ol] B. Oliver, Higher limits via Steinberg representations, Comm. in Algebra 22 (1994), 13811393. MR 95b:18007

$[\mathrm{Pu}] \quad$ L. Puig, Unpublished notes.

[Pu2] L. Puig, Full Frobenius systems and their localizing categories, preprint.

[Qu] D. Quillen, The spectrum of an equivariant cohomology ring: I, Annals of Math. 94 (1971), 549-572. MR 45:7743

[Qu2] D. Quillen, Higher algebraic $K$-theory I, Lecture notes in mathematics 341 (1973), 77-139. MR 49:2895

[Se] G. Segal, Categories and cohomology theories, Topology 13 (1974), 293-312. MR 50:5782

[Sol] R. Solomon, Finite groups with Sylow 2-subgroups of type .3, J. Algebra 28 (1974), 182198. MR 49:9077

[Sw] R. Swan, The nontriviality of the restriction map in the cohomology of groups, Proc. Amer. Math. Soc. 11 (1960), 885-887. MR 23:A1370

Departament de Matemàtiques, Universitat Autònoma de Barcelona, E-08193 Bellaterra, Spain

E-mail address: broto@mat.uab.es

Department of Mathematical Sciences, University of Aberdeen, Meston Building 339, Aberdeen AB24 3UE, United Kingdom

E-mail address: ran@maths.abdn.ac.uk

LAGA, Institut Galilée, Av. J-B Clément, 93430 Villetaneuse, France

E-mail address: bob@math.univ-paris13.fr 